\title{
Nonlinear acoustics of water-saturated marine sediments
}

\author{
Jensen, Leif Bjørnø
}

Published in:

Acoustical Society of America. Journal

Link to article, DOI:

$10.1121 / 1.2003625$

Publication date:

1976

Document Version

Publisher's PDF, also known as Version of record

Link back to DTU Orbit

Citation (APA):

Jensen, L. B. (1976). Nonlinear acoustics of water-saturated marine sediments. Acoustical Society of America. Journal, 60(Suppl. 1), S96-S97. https://doi.org/10.1121/1.2003625

\section{General rights}

Copyright and moral rights for the publications made accessible in the public portal are retained by the authors and/or other copyright owners and it is a condition of accessing publications that users recognise and abide by the legal requirements associated with these rights.

- Users may download and print one copy of any publication from the public portal for the purpose of private study or research.

- You may not further distribute the material or use it for any profit-making activity or commercial gain

- You may freely distribute the URL identifying the publication in the public portal

If you believe that this document breaches copyright please contact us providing details, and we will remove access to the work immediately and investigate your claim 


\title{
The 92nd Meeting of the Acoustical Society of Arnerica
}

Town and Country Hotel

\author{
San Diego, California
}

15-19 November 1976

\author{
Session A. Physical Acoustics I: Atmospheric Acoustics I \\ Edmund H. Brown, Chairperson \\ Wave Propagation Laboratory, NOAA, Boulder, Colorado 80302
}

\section{Invited Papers}

$8: 30$

\begin{abstract}
A 1. Contributions of acoustic echo sounding to atmospheric research and services. C. Gordon Little (Wave Propagation Laboratory, NOAA, Boulder, CO 80302)

This paper reviews briefly the first few years of acoustic remote sensing that followed the introduction of the echosonde in 1966, and then surveys in more detail many aspects of recent developments in acoustic echo sounding. The early, qualitative, facsimile recordings are now been supplemented by attempts to make the echosonde data quantitative. This has involved careful comparison of data from calibrated sounders and instrumented meteorlogical towers. Evidence is presented supporting the claim that echosondes can supplement the facsimile records with height profiles of mean wind speed and direction, as well as profiles of the structure constant of velocity $C_{y}$ and temperature $C_{T}$. The former permits derivation of the height profiles of the turbulent energy dissipation rate $\epsilon$ and the latter the measurement of height profiles of the structure constant of optical refractive index $C_{n}$. Various real $z$ ations of Doppler echosondes are presented, together with an echosonde interferometer which may offer promise of measuring atmospheric temperature profiles. Some future directions for acoustic echo-sounding developments are summarized.
\end{abstract}

\section{Contributed Papers}

9:00

A2. Acoustic sounding of atmospheric winds. D. R. Jensen and J. H. Richter (Naval Electronics Laboratory Center, 271 Catalina Blvd., San Diego, CA 92152)

Acoustic echo sounders have recently come into their own as valuable remote sensors for determining atmospheric structures and winds. Kelton and Bricout (Bull. Am. Meteor. Soc. $45,571-580$ (1964)] first demonstrated that the acoustic Doppler shifted signals from scattered sound were related to the ambient winds. Later, McAllister [J. Atmos. Terr. Phys. 30, 1439-1440 (1968)] showed that a vertically pointing monostatic acoustic sounder could reveal, in intricate detail, the atmospheric boundary layer structure. An acoustic echo sounder and wind sensor system capable of simultaneously measuring temperature fluctuations in the lower atmosphere and the vertical profile of wind speed and direction has been in operation at the Naval Electronics Laboratory Center since 1973. It is the purpose of this paper to describe the capability of this acoustic echo and wind sensor system for determining atmospheric structure and winds.
9:15

A3. Acoustic wind and wind-shear mesururing system. P.A. Mandics and D.W. Beran (Wave Propagation Laboratory, Environmental Research Laboratories, National Oceanic and Atmospheric Administration, Boulder, CO 80302)

An acoustic wind-profiling system designed to detect hazardous wind-shear conditions in the airport environment has been developed during the past four years. The system installed at Dulles International Airport consists of a vertically pointed transmitter surrounded by three receivers $290-\mathrm{m}$ distant and separated by $120^{\circ}$ in azimuth. Electronically steered receiver beams track the upward propagating transmitted tone burst and collect the scattered acoustic signals. The Doppler frequency shift of the returns is analyzed digitally to determine the horizontal wind at 20 height levels in 30-rn increments. Unique design features of the system such as the steered receiver antenna are described. A one-leg prototype of the Dulles system was installed and tested at Table Mountain near Boulder, CO. Winds measured by the prototype acoustic system compared well with those determined by an $F M-C W$ radar and a 
balloon-borne anemometer. Noise generalted by l'ain and surface winds exceeding $16 \mathrm{~m} \mathrm{sec}^{-1}$ proved to be the major limilations for the acoustic system. Preliminary results from the Dulles system are also presented.

\section{$9: 30$}

A4. Acoustic echo sounding of the marinc boundiry linyer. I. R Noonkester, D. R. Jensen, and J.H. Richter (Naval Filectronies Laboratory Center, 271 Catalina Blid., San Dicgo, CA 92152)

Acoustical probing of the lower atmosphere has proliferated since 1968 when McAllister [J. Atmos. Terr. Phys. 30, 1439-1440 (1968) l demonstrated its advantages. Consiclerable acoustic echo soundings have been made in continental regions but echo sounding of the marine boundary layer (MBL) is fragmentary. Many hours of acoustic echo-sounding dat a have been taken since 1974 at a multisensor site on the Pacific coast in San Diego, CA, where the prevailing northwesterly winds carry the MBL over the echo sounder almost continuously. Simultaneous obscrrations of the MBL by the echo sounder, other surface-based remote sensors (e.g., FM-CW radar and lidar) and standard meterological sensors (e.g., radiosondes) have aicled in the interpretation of the ccho-sounder data. These observations have been augmented by observations on the Atlantic coast near Wallops Island, VA. The paper will present some characteristic echo-sounder data of the MBL with meterological interpretations.

\section{$9: 45$}

A5. Acoustic sounding of the tropical marine boundary layer during GATE. P.A. Mandics, J.E. Gaynor, and F. F. Hall, Jr. (Wave Propagation Laboratory, Environmental Research Laboratories, National Oceanic and $A$ mospheric Administration, Boulder, CO 80302)

A vertically pointed monostatic acoustic sounder was installed on the NOAA Ship OCEANOGRAPHER during the Global Atmospheric Research Program Atlantic Tropical Experiment (GATE). The sounder antenna was mounted on a gyrocontrolled plat form to compensate for the ship's pitch and roll motions. Extensive measures such as mounting the intenna assembly on a vibration isolator and installing absorbing cuffs had to be taken to reduce interference by ship-generated noise. Backscattered acoustic data obtained from up to $850 \mathrm{~m}$ height describe the tropical marine boundary layer in unique and hitherto un seen detail. Du ring undisturbed weather conditions the facsimile record showed convective plumes rising from the surface of the water up to $400 \mathrm{~m}$. Storm-generated disturbances resulted in a substantial modification of the boundary layer; low-level, multilayered, undulating inversions formed from cool outflow currents. The inversions persisted for up to 16 hours. Low-level, patchy cumulus clouds produced characteristic hummock-shaped acoustic echoes. Analysis of the Doppler frequency shift of the returns allowed the determination of vertical velocities within these clouds and underlying convective plumes.

\section{$10: 00$}

A6. Observations of buoyant plumes with monostatic acoustic radar. P. B. MacCready, Jr. (AeroVironment Inc., 145 Vista Ave., Pasadena, CA 91107)

Monostatic acoustic radar detects echoes from an artificial heated plume as well as from natural convective plumes; thus it has been used on occasion to ascertain the location, height, and thickness of the buoyant effluent from industrial sources. A brief-field investigation of an industrial source was conducted to compare, for stable lapse rate conditions: (1) the plume heights monitored with a mobile monostatic acoustic radar; (2) heights measured $w$ ith an aircraft when the plume was temporarily marked by smoke, and (3) heights predicted by standard plume rise theory utilizing measured wind, temperature, and turbulence profiles. There was reasonable agreement between all three. Also the acoustic radar verified that the verti- cal thickness of the plume was as latre as that assumed in the plume rise theory (namely, velical thickness comparable to mean plume height above stack lop). lloights predicled using standard plume rise theory, with melerological inputs extrapolated from surface observations ral her that being mealsured aloft, were substantially lower tham the observed heights. The plume lurbulence and temperature gare sufficient acoustic scattering when the plume was mear the linal plume height (about $150 \mathrm{~m}$ ) to avoicl confusion wilh cehoes from the ambient atmosphere.

$10: 15$

A7. Analytical studies of sodar observalions at Jelhi. S. P. Singal, N. N. Datta, B.S. Gera, S.K. Aggarwal (National Physical Laboratory, New l)olhi-110012, Inclial).

A monostatic sodar system has been operating at the National Physical Laboratory, New Delhi $\left(28.6^{\circ} \mathrm{N}, 77.2^{\circ} \mathrm{F}\right)$ since December 1974. Echograms obtained up to Nay 1976 (operational period of approximately $1500 \mathrm{~h}$ have been qualitatively analyzed with respect to their structural detilils. Layers are a general feature of the sodar colograms with their thickness lying mostly within $20 \mathrm{~m}$. Their pereenlige occurrence reaches a maximum of $95 \%$ between $1600-2000 \mathrm{~h}$ and a minimal of $40 \%$ between $1200-1400 \mathrm{~h}$. The height of the layers has a primary maximum (average boight $220 \mathrm{~m}$ and maximum height $350 \mathrm{~m}$ ) between $0200-0400 \mathrm{~h}$, a minimum (average height 175 $\mathrm{m}$ ) between $0800-1000 \mathrm{~h}$ and a secondaly maximum (average height $190 \mathrm{~m}$ ) between $1100-1400 \mathrm{~h}$. 'l'urbulence is a very characteristic observalion under stable conditions and is present for about $1: 3 \%$ of the lime. The lurbulent as also the layer structures have been classified into various categories according to their typical fcalures. Discernible thermal plume structures have been rarely seen over Delhi. 'T'he st mucture is mostly affected by the prevalent ambient wind. The convective activity is maximum around $1200-1400 \mathrm{~h}$.

\section{0:30-BREAK}

\section{$10: 45$}

A8. Near-horizontal propagation of sound over grassland. J. F. Piercy, R.J. Donato, and T.F.W. Fmbleton (Division of Physies, National Research Council, Ottaw'a, Ontario, Canada K1A OS1)

An extensive set of published measurements of the propagation of noise from jet aircraft on the ground across an airport for differing weather conditions is compared with predictions using modern propagation theory from values of ground impedance, assuming an acoustically homogeneous atmosphere. When the atmosphere is in fact reasonably homogeneous under neutral weather conditions, there is quantitative agreement between calculated and measured behavior. The intricate pattern of acoustic behavior, as a function of frequency and distance, produced by inhomogeneous atmospheric conditions (gradients of wind temperature turbulence) is explained qualitatively by the appropriate modes of propagation revealed by the theory. The role of surface and sky waves in particular is illustrated.

\section{$11: 00$}

A9. Results from an acoustic sounder network study of the San Francisco Bay Area. P.B. Russell and E. E. Uthe (Stanford Research Institute, Menlo Park, CA 94025)

This study, begun in 1974, was expanded to a small network of three stations in 1975, with some additional data received from two sites operated by others. The measurements indicated widespread (up to $60 \mathrm{~km}$ inland) and frequency occurrence of a low $(200-700 \mathrm{~m})$ marine inversion, which was easy to detect acoustically. Records from San Jose could be usefully classified by the scheme developed in 1974 for Stanford-Menlo Park. They confirmed that the time of transition from nearsurface stability to convection and the height of the inversion aloft (both as depicted by the sounder records) were equally important predictors of near-surface primary pollutant con- 
centration. Records from Mt. Sut ro (San F ranciseo) repeatedly showed a very intense inversion with the characteristic diumal height variation (]ate afternoon minimum) revealed by a simultaneous direct-sensing study $[A$. Miller, Bull. Am. Meteor. Soc. 56, 52-55 (1975) /. Temperature profiles measured by tower, remotely piloted aircraft, and balloon at each site comfirmed the inversion and convection behavior depicted by the sounder records. A 1:3-station network is starting operation in August 1976, to provirle input data for an operational computer model of Bay A re:a ais pollution. Preliminary results will be shown.

$$
\text { 11:15 }
$$

A10. Detailed evaluation of monostatic acoustical sounder determinations of urban atmospheric boundary layer structure. J. L. MeElroy and A. M. Pitchford (U.S. Environmental Protection Agency, EMSI-LY, P.O. Box 15027, Las Vegas, NV 89114)

Field programs for the development and evaluation of air quality simulation models and for the design of optimum air monitoring networks are being conducted over Metropolitan St. Louis, Missouri, and Las Vega. Nevada, respectively. As part of these programs, urban boundary layer structure (principally mixing depth) is monitored using monostatic acoustical sounders; free, tethered, and radio-controlled radiosonde flights; aerometric measurements from vertical spirals with helicopter and fixed wing aircraft; and, return signatures from ground-based and airborne dounlooking LIDAR. In this paper, extensive comparisons are made between acoustical sounder information and that using the conventional in situ and other remote techniques. Stratification of data is made according to distinctive segments of the diurnal boundary layer cycle, overall synoptic weather situation, sounder pattern recognition type, and site location (i. e., climatic region). In general, the data sets compare well and the overall results are similar to previous limited studies. Differences are largely relatable to the underlying physical principles of operation and to the response-resolution properties of the various techniques.

$$
\text { 11: } 30
$$

A11. Detection of acoustic backscatter from falling snow by acoustic Doppler sounding. H. Melling and R. List (Depart- ment of Physics, University of Torcnto, Toronto, Canada M5S (A7)

Acoustic echoes have been detected during medium intensity snowfall by a verticilly directed acoustic Doppler sounder. The echo was observed at a level in excess of $30 \mathrm{~dB}$ above noise. When displayed on a facsimile recorder the signal was mottled and diffuse in character, showing a decrease in intensity with height. When compensation was made lor atmospheric attenuation the echo intensity was quite uniform with height. Yertical velocities meanured by the sounder w'ere downward and averaged $1-1.5 \mathrm{msec}^{-1}$. The standard deviation was $0.8-1 \mathrm{msec}^{-1}$. These values agree with concurrent optical estimates of snow fallspeed. A comfutation of the seattering cross section from the echo intensity is in agreement with empirical relationships deduced for electromagnetic radars. Statistics of "instant aneous" echo frequency and power were also computed. Only the probability density function of power did not agree with the predictions of Srivastava and Carbone [Radio Science 4, 381-393 (1969)]. These authors predict an exponential probability density for power whereas the observations statisfy a log-normal function. This result suggests that multiple scattering may be of signifi sance in acoustic sounding.

11:45

A12. Echosonde detection of the abrupt transition from a laminar to a turbulent planetary boundary layer. J.F. Schubert (Savannah River Laboratory, E. I. du Pont de Nemours and Company, Aiken, SC 29801)

Echosonde measurements of the akrupt transition from a laminar to a turbulent planetary boundary layer are compared with meteorological measurements from a 300-m TV tower and a network of seven $61-\mathrm{m}$ towers located near the echosonde site. Preliminary data show that the conditions necessary for early nighttime transition from laminar to turbulent flow exist during clear afternoons when the heat flux changes sign, the planetary surface begins to cool, and the surface wind dies. [Work done under USERDA Contract No. AT(07-2)1.]

\title{
Session B. Psychological and Physiological Acoustics I and Underwater Acoustics I. Animal Sonar Systems: Current Investigations
}

\author{
R.A. Johnson, Chairperson \\ Naval Undersea Center, San Diego, California 92132 \\ Invited Papers \\ 9:00
}

B1. Echolocation ontogeny in the pallid bat (Antrozous pallidus). Patricia E. Brown (Department of Biology, UCLA, Los Angeles, CA 90024)

The ability to heat and emit the ultrasounds necessary for echolocation is not present at birth in the pallid bat and does not appear until at least a week postnatally. Dramatic changes occur both in pulse structure and auditory tuning as the bat matures. Pallid bats begiri to fly at four weeks of age, but continue to nurse until seven weeks old. This allows them time to coordinate the flight and echolocation skills necessary for survival. Bats learn to correlate outgoing pulse with returning echoes. Naive bats approaching an obstacle do not exhibit the 
expected increase in pulse repetition rate, which occurs after some expericnce. The normal development of echolocation skills as judged by obstacle avoidance can be correlated with sound emission. Deficits occur in bats raised in environments in which their echoes are distorted or attenuated.

B2. Neurophysiological studies on the echolocation system of bats sensitive to Doppler-shifted echoes. N. Suga, G. Schuler, and P.H.-S. Jen (Department of Biology, Washington University, St. Louis, MO 63130)

Rhinolophus ferrumequinum and Pteronotus parnellii emit orientation sounds consisting of a long CF component and a short FM component. The CF component is used for "IDopplershift compensation." In rf, the gate used to obtain the frequency information for the compensation is probably opened at the beginning of the sound emission and is closed shortly after the end of the emission. The cricothyroid muscle is highly developed and its activity decreases proportionately with the amount of Doppler-shift compensation. These bat $s$ have peripheral auditory systems highly specialized for the reception of the CF component in orientation signals. The extent of cortical representation of the peripheral sensory field depends on its important for species behavior. In p.p., neurons processing the CF components in the orientation signal and Doppler-shifted echoes occupy a disproportionately large part of the auditory cortex. The separate FM signal processing area is also large. This disproportionate cortical representation related to features of biologically significant signals is comparable to that found in the somato-sensory and visual systems in many mammals. [Work supported by NSF.]

B3. Acoustic reflexes of middle-ear and laryngeal muscles in the FM bat Myotis lucifugus. Philip, H. -S. Jen, Joachim Ostwald, and Nobuo Suga (Division of Biological Sciences, University of Missouri, Columbia, MO 65201 and Department of Biology, Washington University, St. Louis, MO 63130)

In Myotis lucifugus both the middle-ear muscles (MEM's) and the laryngeal muscles (LM's) are highly developed for echolocation. The se muscles respond to acoustic stimuli with a shortest latency of 3 and $6 \mathrm{msec}$, respectively. The tuning curves of the MEM fibers are broader than those of the LM fibers. The lowest threshold of the acoustic reflex of the MEM's is $20 \mathrm{~dB}$ lower than that of the LM's. The majority of the MEM and LM fibers are tuned to sounds between 35 and $45 \mathrm{kHz}$. On the average, the LM fibers are $5 \mathrm{~dB}$ more sensitive to 4-msec FM signals sweeping downward $10 \mathrm{kHz}$ across their BF's than to pure tones, but this is not true for equivalent upward sweeping FM signals. On the other hand, the MEM fibers show poorer responses to FM signals than pure tones, regardless of sweep direction, Electrical stimulation of the superior laryngeal nerve evokes action potentials in the MEM's with a shortest latency of $5 \mathrm{msec}$. The LM's and MEM's are apparently coupled through sensory fibers in the superior laryngeal nerve. [Work supported by University of Missouri summer research fellowship, Studienstiftung des deutschen Volkes, and NSF Grant 75-17077.]

10:00

B4. Characteristics of phasic "on" neurons in the inferior colliculus of unanesthetized bats with observations relating to mechanisms for echo ranging. G. D. Pollak, D.S. Marsh, R. Bodenhamer, and A. Souther (Department of Zoology, University of Texas, Austin, TX 78712)

Single units with phasic "on" firing patterns were recorded from the colliculus of unanesthetized bats (Tadarida brasiliensis mexicana). Our intention was to identify a population of neurons whose response characteristics are suitable for measuring the time interval between an emitted pulse and echo and, therefore, would be of value for echo ranging. Three catagories of units are distinguished on the basis of the consistency of the initial spike latent periods elicited by repetitive signals. Most important are the "phasic constant latency responders" (PCLR) in which the dispersions of initial spike latencies were at most \pm 250 $\mu \mathrm{sec}$ at any intensity and where the mean latency charged by at most $1.7 \mathrm{msec}$ when the signal level was increased from 3-6 dB to $30-40 \mathrm{~dB}$ above threshold. The initial spike latencies in most PCLR's were erratic with tone bursts but became highly synchronous with frequency-modulated (FM) signals resembling the natural chirps of this species. Each PCLR had a "hair trigger" threshold to FM signals where the firing probability rose from near 0.0 to $0.8-1.0$ as the signal level was increased from threshold to $2-6 \mathrm{~dB}$ above threshold. In addition, each of these units exhibited an "independent recovery" pattern where the presentation of an initially loud pulse did not effect the response to a simulated echo following shortly thereafter. The response and recovery characteristics of the PCLR's were correlated with behavioral data reported in previous studies and it is shown that the PCLR's have the capability for measuring the time interval between a pulse and the returning echo. Moreover, by assuming that the bat can correct for inherent "errors" resulting from dissimilar pulseecho intensities the population of PCLR's can provide a measurement of target distance that becomes progressively more accurate as the bat homes in on its target. [Supported by NSF Grant P401855-000. 1 
10:20

B5. Representation of target range in the sonar receivers of echolocating bats. James $A$. Simmons and $W$. A. Lavender (Department of Psychology and Program in Neural Seiences, Division of Biological and Biomedical Seiences, Washington University, St. Louis, MO 6.31:30)

Echolocating bits can discriminate the range (echo arrival time) of simulated targets with accuracy predictable directly from the sonar waveforms down to the region where ectio and noise powers are about equal. Detection and ranging performance are closely linked. Equivalence of performance in simultaneous- and successive-presentation procedures indic tes that bats make absolute range judgments and store range images of targets for subsequent comparisons. Targels separated in range and/or angular direction are perceived as distinct events. Although target range is perceived noncoherently, target fine structure maly te perceived coherently. Information about the occurrence and timing of transmitted sounds and echoes is conveyed in the time domain over frequency-speeific primary auditory neurons and appears, still in the time domain, ascending the lateral lemniscus and in the inferior colliculus. Behavioral data suggest ultimate spatial "receptor-field" representation of range in the brain, so echo-timing information may be recoded into range-specific neurons at or above the inferior colliculus. The data generally suggest that target range is processed by channels used for periodicity-pitch perception in nonecholocating animals. [Work supported by NSF. 1

$10: 40$

B6. Range resolution by an echolocating bottlenosed dolphin (Tursiops truncatus). A. Farl Murchison (Naval Undersea Center, Hawaii Laboratory, P. O. Box 997, Kailua, HI 967:34)

Utilizing a two-alternative, forced-choice (simultaneous stimuli presentalion) procedure, the ability of a bottlenosed dolphin (Tursiops truncatus) to discriminate differences in target range by echolocation was determined. Target-range difference discrimination $t$ rials were conclucted at three different absolute ranges $(1,3$, and $7 \mathrm{~m})$. The range resolution acuity of the dolphin varied with absolute range. His 0.79 hit rate probability for the absolute range of one $\mathrm{m}$ was $0.9-\mathrm{cm}$ range difference; for the absolute $r$ ange of $3 \mathrm{~m}$ it was $1.5 \mathrm{~cm}$ and $\mathrm{lor}$ an absolute range of $7 \mathrm{~m}$ it was $2.8 \mathrm{~cm}$. Behavioral data suggest that these levels of perceptual acuity are not usually attained and/or maintained during the dolphin's normal nonstatic prey pursuit. [Work supported by NUC.]

\section{$11: 00$}

B7. Periodicity pitch difference limens in the bottlenose dolphin (Tursiops truncutus). R. H. Defran and Naney G. Caine (Department of Psychology, San Diego State University, San Diego, CA 92182)

An Atlantic bottlenose dolphin (Tursiops truncritus) was trained to differentiate between similar streams of sonarlike pulse pairs. The interval between pulses of a pair was of primary interest and was varied from trial to trial. All signal parameters were designed to simulate the emitted signals and returned echoes present in a dolphin sonar task. Discrininative responding to variations in the interpulse interval allowed for an estimation of the dolphin's temporal diserimination ability. Psyehophysieal functions constructed from the data were orderly, asymptotic, monotonically related to the duration of the comparison intervals, and statistically reliable. Psychophysical thresholds, defined as the $75 \%$ correct level of responding, indicated that the dolphin was capable of resolvins differences as fine as $100 \mu$ sec. Further, the results confirmed that the dolphin's temporal discrimination is most accurate for pulse pair intervals simulating the shortest distances. Results were interpreted within the framework of a periodicity pitch analysis. Extrapolation of the data suggest that sonar discrimination of distince in the dolphin involves a complex frequency discrimination rather that a correlation diserimination.

$11: 20$

B8. Echolocation diserimination of complex planar targets by the Beluga whale

(Delphinapterus leucas). V.S. Gurevich (Hubbs-Sea World Research Institute, San Diego, CA) and W. E. Evans (Naval Undersea Center, San Diego, CA 92132)

Results of a study of a Beluga trained to diseriminate between two complex planar targets, indicate that this animal's echolocation discrimination capability with and without blinc folds is superior to that of bottlenosed dolphins tested on similar targets. The standard target used was a three-step pyramid constructed of three polyvinyl chloride sheets each $13 \mathrm{~mm}$ thick. The first step was $10 \times 10 \mathrm{~cm}$, the second step was $7 \times 7 \mathrm{~cm}$ and the third step wis $3 \times 3 \mathrm{~cm}$. The selection of the standard target was considered a correct response. The comparison targets (ineorrect choice) differed from the standard target only in the size (surface area) of the third step. With a comparison of a third step of $2.9 \times 2.9 \mathrm{~cm}$ versus the standard, $3.0 \times 3.0 \mathrm{~cm}$, the animal's mean correct response was $80.8 \%$ correct. When presented with two standard or identical targets the mean response dropped to ehance (55. $5 \%$ ). The performance of the bottlenosed dolphins tested on similar targets dropped to chance on a comparison of $2.7 \times 2.7 \mathrm{~cm}$ versus $3.0 \times 3.0 \mathrm{em}$. The pulses used by the Beluga during the echolocation discrimination task contain very little acoustic energy below $12 \mathrm{kHz}$ with the 
peak energy occurring at 40,80 , and $120 \mathrm{kHz}$. The measurements of the frequeney eomponents in the echolocation signal above $110 \mathrm{kHz}$ are only tentatively believed due 10 limitations in the frequency response characteristics of the hydrophones used. The ability to use both sensory channels (tests without blindfolds) appeared to enhance discrimination ability slightly. [Work supported by NUC.I

$11: 40$

B9. Propagation of dolphin echolocation signals. Whitlow W. L. $\Lambda$, Rubert W. Floyd. and Jeffrey E. Haun (Naval indersea Center, Kailua, HI 96734)

The propagational characteristics of high-frequency echolocation signals (peak energies above $100 \mathrm{kHz}$ ) of the Atlantic bottlenose dolphin (Tursiops truncatus) in open witers were determined by a series of measurements conducted while the animal performed a largetdetection task. The animal was trained to station in a "chin-rest" device so that the vertical beam pattern could be measured with a vertical array of hydrophones and the horizontal beam pattern could be measured with a horizontal array. The animal's signals as a function of distance from the animal out to $2 \mathrm{~m}$ were measured with a horizontal array of hydrophones placed directly in front of the animal. These measurements were used to study the relationship of the signals in the nearfield and farfield of the animal's sonic beam. The relationship of the signals measured with a contact hydrophone placed on the animal's - rostrum and on the melon with the signals measured at $1 \mathrm{~m}$ and at the target wals also studied. It was found that the 3-dB beamwidth was approximately $8.5^{*}$ for both the horizontal and vertical beams. The major axis of the vertical beam was direeted at an angle of $20^{\circ}$ above the plane defined by the animal's teeth. It was also found that the fartield bogins at approximately $0.5-0.6 \mathrm{~m}$ from the tip of the animal's rostrum.

\section{Contributed Papers}

$12: 00$

B10. Effects of stimulus duration upon the response characteristics of single neurons in the inferior colliculus of unanesthetized bats (Tadarida brasiliensis mexicana). D.S. Marsh, G. D. Pollak, and R, D. Bodenhamer (Department of Zoology, University of Texas, Austin, TX 78712)

Constant frequency (CF) and frequency-modulated (FM) pulses were utilized to examine the response characteristics of collicular neurons. Tonic, toniclike and various types of phasic "on" responses were noted depending upon the type of stimulus used. For example, a tonic response patten elicited from a given neuron with $\mathrm{CF}$ stimulation could be changed to a phasic "on" pattern with FM stimulation. Similarly, thresholds and input-output functions were affected by the stimulus type. Superimposed upon the effects of stimulus type were the effects of stimulus duration. Many neurons were found to have firing patterns and response characteristics to long duration stimuli that differed greatly from those observed with shorter signals having durations less than $2.0 \mathrm{msec}$. Results suggest that most inferior collicular neurons are best suited for extracting information from signals having durations comparable to those emitted during the search and approach phases of goal-directed flight and are poorly suited for responding to shorter signals having durations typical of those emitted during the terminal phase of echolocation. [Supported by NSF Grant P401855-000.1

$$
\text { 12:08 }
$$

B11. Signal duration and its effect on recovery character istics of single units in the inferior colliculus of unanesthetized bats. R. Bodenhamer, G,D. Pollak, and D. S. Marsh (Department of Zoology, University of Texas, Austin, TX 78712)

Recovery cycles were obtained from single units in the inferior colliculus of awake Mexican free-tailed bats (Tadarida brasiliensis mexicana) by recording responses to simulated pulse-echo pairs. The pulse-echo pairs were designed to mimic natural outgoing cries and returning echoes, and were either constant frequency tones or, as in most instances, frequencymodulated tones. Since echolocating bats systematically vary pulse duration as they progress towards a target, we asked what effects these duration changes might have on neuronal recovery. Therefore, the durations of the simulated pulse and echo were varied from 1-10 msec and recovery observed with several signal durations. Even at short interpulse intervals and with initial pulses $25-50 \mathrm{~dB}$ more intense than the following "echoes," the majority of units receovered well when signal durations were $3.4 \mathrm{msec}$ or longer. Recovery with signal durations of less than 2,0 msec. however, was typically poor. The results indicate that individual units may show strikingly differ ent recovery patterns when durations of the stimulating pulse and echo are changed. This suggests that some units of the colliculus may only be encoding incoming information during restricted segments of the echolocation cycle, or alternatively, that single units may be involved in encoding different types of information during different segments of the echolocation cycle. [Supported by NSF Grant P401855-000.] 


\title{
Session C. Noise I: Land Use Planning
}

\author{
Simone L. Yaniv, Chairperson \\ National Bureau of Standards, Washington, D.C. 20234
}

Invited Papers

$9: 00$

C1. Overview of land use planning. M. Pepin-Donat (Office of Planning and Program Coordination, Department of Housing and Urban Development, Washington, DC 20410)

A new increasing awareness of the finite quality of land as a resource exists in the I'nited States today. This change in perspective is challenging traditional practices resulting :n conflicting values in American society. The issue of land use planning is extremely complex and influences by a multitude of independent yet interrelated factors such as government-local, State, and Federal, the citizen; the developer; the courts; industry and technology; and banking. Tradiationally, the right to plan and control land in the United States rests with each individual State. There are a minimum of 160 Federal programs-grant, loan, and regulatory, which impact land use in addition to individual State and local programs. A variety of tools and mechanisms have been developed to resolve the complex land use problem. Coordination is sought in all 50 States through an A-95 Clearinghouse mechanism and supplemental state programs. The courts are taking an increasingly active role in influencing the direction of land use planning. An attempt to relate recently enacted or proposed noise control legislation to these land use processes will be included.

$$
9: 30
$$

C2. Airports and land use planning. S. E. Starley (Office of Noise Abatement and Control United States Environmental Protection Agency, Washington, D. C. 20460)

The EPA has developed an Airport Noise Regulatory concept, including an impact analysis methodology and a readily understandable format for the presentation of results. The regulatory process provides a means of reducing and/or preventing aviation noise impacts vi; a balanced mix of aviation actions and land use controls. The analysis methodology which EPA has developed is based upon a comparison of aviation noise and the background levels which are due to all sources other than aviation. Hence, the noise impacts of multi modal systems can be evaluated. Noise criteria have been developed for the classification of airports, by sever ity of impact, and for goals necessary to protect the public health and welfare. The regulatory process was designed to insure participation of the airport proprietor, local governments, and the general public. Each airport noise abatement plan will be subjected to public scrutiny, in an open hearing, as well as to review by the Federal Aviation Administration, with consulation by the EPA. The process results in an airport operating plar. for noise abatement which is basic to land use planning.

$$
10: 00
$$

C3. Influence of noise control on land use planning. C. R. Bragdon (Department of City Planning, Georgia Institute of Technology, Atlanta, Georgia 30322)

City planning has been an established professional discripline for nearly 50 years in the United States. Evolved from architecture and other physically related disciplines city planning has broadened to also include interest in social and environmental concerns. Zoning was the first land use planning technique to incorporate noise performance requirements. Today many other land use planning techniques consider noise, among other environmental factors. An inventory of these planning techniques and their relative success are discussed, drawing upon municipal, state and Federal experiences. Recommendations are made for future courses of action to encourage greater effectiveness.

$$
10: 30
$$

C4. Overview of California's efforts in the area of planning for noise compatible land uise. R. R. Illingworth and R.B. DuPree (California Department of Health, Office of Noise Control, 2151 Berkeley Way, Berkeley, California 94704)

This paper reviews the role of the California State Government in noise compatible land use planning. Since the responsibility for land use planning in California lies almost entirely with local government, the State has provided, through various pieces of legislation, the means for insuring that noise is considered in the process. Among the approaches the istate has adopted are (1) a requirement that cities and counties adopt a noise element as part of their general plan; (2) the establishment of an Office of Noise Control to provide assistance to local governments and coordinate efforts at the State level; (3) the establishment of air- 
port land use commissions; (4) the use of the EIR/EIS review process; and (5) the adoption of noise insulation standards for multifamily dwellings. The effectiveness of these measures in achieving noise compatible land use is discussed. Examples of good and that land use in the vicinity of various sources are presented and analyzed. Some alternative approaches are presented and pitfalls pointed out.

\section{Contributed Papers}

$11: 00$

c5. Significance of land use elements in developing noise maps. Basil H. Manns (Environmental Protection Agency, Office of Noise Abatement and Control, Washington, DC 20460) and Sharon B. Holcombe, and Robert L. Hershey (Booz, Allen Applied Research, 4733 Bethesda Ave., Bethesda, MD 20014)

The procedure used to relate noise level to land use is basically through a simple function of population density. The 100-site study conducted by the U.S. EPA in 1973 attempted to verify the premise that outdoor noise does correlate with population density. The function derived has been used extensively in airport planning to estimate the indigenous noise in a community. However, extreme care must be taken in using the results of this 100 site study to develop noise maps directly, since they exhibit wide scatter as a function of various land use elements. The uncertainty of the procedure and the significance of seemingly insignificant parameters have been investigated (show actual sites variation). An algorithm has been developed to estimate nois - jevels at any given point in a community on the basis of land parameters. This algor ithm involves the construction of artit hall trafic noise levels using the population density and the rodd configuration. The algorithm can be expanded to prepare noise maps by using clusters of homogeneous land use elements. Emphasis is given to surface transportation noise sources rather than aircraft operations, since this type of noise impacts more people than any other single category. A discussion is also included as to how a planner or designer may use such a statistical procedure in estimating community noise. The algorithm may be used by a planner, together witb noise models of airports, rapid transit systems, and industrial sites, to produce comprehensive noise maps. Examples of noise maps are presented for the Washington Technical Institute complex in Washington, D.C. , 1 the Los Angeles Airport area, and the Lindberg Field area in San Diego.

\section{$11: 15$}

C6. Designing a community noise regulation. L.S. Goodfriend, E. M. Clark, and S. M. Ober (Lewis S. Goodfriend and Assoeiates, 7 Saddle Road, Cedar Knolls, NJ 07927)

The officials of the Township of East Brunswick, New Jersey, set out to establish a noise control regulation for their municipality that would meet the health and welfare needs of its residents and still provide a suitable climate for business and industry. East Brunswick is a municipality of 23.5 square miles, having a residential population of 36790 , and land uses including farming, industrial plants, strip commercial zones along a major state highway, and a major turnpike. Major steps of the program to develop a regulation were physical examination of the township and its land uses; review of the existing master plan; determination of the health-related concepts of noise exposure; development of a rationale for selecting a noise descriptor permitting enforcement by suitably trained local officials; determination of present ambient sound levels; and development of the regulation structure, limits, and enforcement techniques. The new regulation is based on octave band levels not to be exceeded at the boundaries of each of three land use classes formed from groups of existing land use zones. Where sounds are intermittent, duration-level tradeoffs are permitted up to a limit of $15 \mathrm{~dB}$.

$$
\text { tit: }
$$

$11: 30$

C7. Assessment of noise impact in transportation planning. S. Fidell (Bolt Beranek and Newman Inc ${ }^{\circ}$, P. P.: Box 633.
Canoga Park, California 91305)

As part of a comprehensive reference book on environmental impacts in transportation corridors, a practically oriented guide to assessment of noise effects has been prepared for the California Department of Transportation. Among the major areas covered are (1) review of current knowledge of noise and vibration effects associated with highway and rail traffic; (2) provision of methods of estimating exposure levels associated with varying amounts of traffic; (3) relation of expected levels of exposure to anticipated social impacts; (4) explanation of a quantitative Noise Impact Index for evaluating alternative routes; and (5) discussion of measures to mitigate noise impacts. This paper summarizes the findings contained in the guide to noise impact assessment to bring it to the attention of transportation planners and other's concerned with assessment of noise effects.

\section{$11: 45$}

C8. Workship on noise control and urban planning. Donna McCord Dickman (Metropolitan Washington Council of Governments, 1225 Connecticut Ave. N. W. , Washington, DC 20036) and Patrick Anderson (EPA, Region III, 6th St. and Walnut St. , Philadelphia, PA 19106)

In recognition of the fact that unrestricted land development and planning decisions made without regard to noise have left a legacy of noise problems which are just now being addressed by nolse abatement progams, the Metropolitan Washington Council of Governments and the Environmental Protection Agency, Region III, sponsored a two day Workshop on Noise Control and Urban purpose of the workshop was

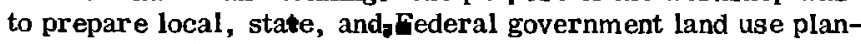
ners to identify and quantify potential noise problems using current analytical techniques and solutions to noise problems through compatible land use planning. The Workshop was divided into several major elements. Problem definition, assessment alternatives and problem solutions presented the land use planner with a logical, sequential path of attack. A discussion of the integration of Federal, State, and local legislative parameters into the planning process was coupled with the latest analytical techniques and noise prediction programs. Potential solutions to the noise problems included planning as well as operational controls. Comprehensive and master planning, zoning, architectural controls, building codes, and capital improvement planning were among the solutions covered. The purpose of this paper is to present highlights of the major elements of the Workshop. 


\title{
Session D. Architectural Acoustics I: Room Acoustics and Sound Isolation
}

\author{
Ronald L. McKay, Chairperson \\ Bolt Beranek and Newman, Inc., Canoga Park, California 91305
}

\section{Contributed Papers}

9:00

D1. Lecture room in Ilunt tIall (formerly the Fogg $\Lambda$ it Museum), Harvard University-An historical review. Ewart $A$. Wetherill (Bolt Beranck and Newnian Inc., Camb ridge, MA 021:38)

At the 96th mecting of the Acoustical Society of America, the author reviewed the history of Hunt $\mathrm{Hall}$-the building where W.C. Sabine began his studies of arehitectural acoustics-ind reported his acoustic measurements of the lecture room immediately before its demol ition in June 1973. Subsequent investigation has answered some unresolved questions and has yielded further information, summarized here.

\section{9:30}

D2. Concert hall meatsurements during musical performances. M. R. Schroeder and W.J. Möller (Drittes Physikal isches Institut, Universitit Göttingen, F. R. Germany, and Bell Liboratories, Murray Hill, NJ 07974)

Most acoustical measurements of eoncert halls are made in unoccupied halls. This paper describes how to use the music signil, recorded by microphones on the stage during a musical performance, as a test signal to measure reverberation time, early and late energies ("clarity"), modulation transfer functions, pronounced echoes and other perceptually important aspects of the hall's impulse response.

\section{9:45}

D3. Unique Properties of an Open-Plan Auditorium. Rein Pirn and Jeffrey $E$. Bollinger (Bolt Beranek and Newman Inc., 1740 Ogden Ave., Downers Grove, IL 60515)

An architecturally open concept was adopted in the design of a 500-seat auditorium for a new school. The audience is seated on four shallow tiers that are adaptable for dining. The space is distinguished by a tall well over the forward seats, beyond which the ceiling is very low. At the back and sides, the arena is open to the student commons. Above and behind the stage, it is open to a spacious mezzanine. Deliberate acoustical provisions include absorptive finishes, lockers that act as barriers, a retractable curtain in place of a rear wall, reflectors to help stage-audience communication, and controlled background noise. Investigations reveal several unique properties: short but remarkably uniform reverberation times, averaging $0.72 \mathrm{sec}$; distance-depedent loudness that imparts a sense of dissimilar room size, depending on seat location; excellent intelligibility of stage sounds, with an average AI of 0.91 for raised voice; and excess attenuation of extraneous sounds on the order of $10 \mathrm{~dB}$.

\section{0:00}

D4. Image method for efficiently simulating small-room acoustics. J. B. Allen and D. A. Berkley (Acoustics Hesearch Department, Bell Laboratories, Murray Hill, NJ 07974).

Image methods have been successfully used in the analysis of the acoustic properties of enclosures for the purpose of architectural design. In this paper we disuss use of image techniques for simulating the impulse response between two points in a small room. The resulting response is then convolved with any desired input signal and the reverberated out- put used for signal processing or psychoacoustic studies. The entire process is earried out using a digital computer so that wide ranges of parameters can be simulated with perfect control over experimental conditions.

\section{0: 15}

D5. Sound transmission between absorbing parallel planes. C. J. Hurst and L. D. Mitchell (Department of Mechanical Engineering, Virginia Polytechnic Insititute and State University, Blacksburg, VA 24061)

Partially absorbing parallel surfaces can be dominant acoustical feature of many rooms. The sound fields in such rooms are not diffuse, which causes difficulty in predicting sound pressure levels. A method is develaped for predicted sound pressure levels in these rooms. It rests on the prediction of sound pressure levels eaused by a nondirectional source of known sound power radiating between absorbing parallel planes. The development proceeds from a geometrical acousties viewpoint. Good correlation has been found between predicted and measured levels in existing, rooms having simple geometries. [Work supported by NSF.]

10:30

D6. Perceptual factors of small room reverberation. Barbara MeDermott and Jont Allen (Bell Laboratories, Murray Hill, NJ 07974)

Room reverberation has a well recgnized characteristic quality which can be annoying in a conference telephony environment. The subjective factors of reverberent speech were studied by asking listeners to judge speech samples in which the short-term and long-term reverberation times the independent variables. Synthetic room impulse responses were generated on a digital computer based on an image model of a room. Four microphone-loudspeaker distances were combined with four wall adsorption coefficients, giving a total of 16 distinet room conditions. Speech samples consisting of ten sentences spoken by each of four talkers were reverberated by the $\mathbf{1 6}$ room conditions. All possible pairs of the 16 room conditions we re presented to listeners for judgment. The subjects were asked to use a scale from zero to nine to report how different they thought the speech sounded in each pair of rooms. These data were analyzed by a scaling procecture that relates the difference judgments to a distance in a multidimensional perceptual space (INDSCAL). Our success in relating physically measured variables derived from the impulse responses to the factors of the perceptual space will be discussed.

$10: 45$

D7. Acoustic environment for audio teleconferencing. $R$. Botros (Bell-Northern Research, P.O. Box 3511, Station C, Ottawa, Ontario, Canada K1Y 4H7)

In contrast to handset telephony, the acoustic environment plays an important role in handsfree (loudspeaking) telephony and more significantly in audio teleconferencing. With increasing distance between talker and telephone terminal, the effects of room resonances, reverberance and background noise on the quality of speech telecomraunication became more and more critical. The range of variation of these parameters 
in executive offices and conference rooms was investigated in this survey. 'The results indicate a common shortcoming in most of these locations: a sound-absorbing ceiling and hard, parallel walls. The effectiveness of directional microphones in improving the situation was subjectively tested in simulated environments. The results gire an indication of the limits of acceptability of the acoustic environment. A weighting sy stem for ranking the audio quality of a conference room is proposed, taking into consideration the above mentioned factors and results. [l'ork supported by Bell Canada. ]

$$
\text { 11:00 }
$$

D8. Acoustical design of a major international airport. R. E. Nugent, C.M. Salter, and J.C. Freytag (Bechtel Corporation, P.O. Box 3965, San l'ranciseo, CA 94119)

The application of noise-control engineering during the design of a new international airport for a future population center presents unique challenges. This ai rport will be constructed on a site $25 \mathrm{~km}$ long and $12 \mathrm{~km}$ wide and is $22 \mathrm{~km}$ from any populated area. The airport faeilities include two international terminals, two domestic terminals, a large mosque, a control tower, a royal facility, and many support buildings. This paper discusses the organization and procedures used to provide noise-contrul engineering support to the engineering and architectural firms designing these various facilities. Included is the methodology used in determining the acoustical eriteria, the sound isolation and speech privacy requirements for the various spaces. Sound isolation details and unit cost comparisons are presented.

\section{$11: 15$}

D9. Recent experience with the California Insulation Standards. John K. Hilliard and Gordon L. Bricken (Bio-Acou stical Engineering Corp. Tustin, CA 92680)

The California Noise Insulation Standards have been in effect for about two years. This unique piece of legislation has for the first lime placed minimum requirements for noise insulation within all building codes in the state. Many such modifications of the building code takes time to filter down to the local governments. Lacking the acoustical expertise, local govemments have rarying responses to the Noise Insulation Code and varying approaches to responsibility for imposing requirements. One resalt of the eode has been stricter review's of current practices in constructing party walls and floor-ceiling assemblies in the field. This has revealed many difficulties in eurrent designs and most particularly in practices in the building indust ry for the installation of such assemblies. This paper will address the experience of Bio-Acoustical Engineering Corp. als a consultant to builders and local building offieials on this issue in over 500 different separate reports in the last two years. Some recommendations on the implementation of the code will be included.

D10. Simplified method of party-wall testing. John K. Hilliard and Gordon L. Bricken (Bio-Acoustics Engineering Corp., Tust in, CA 92680)

The California Noise Insulation Standards has for the first time imposed STC testing on a bmad segment of the building inclustry. Local officials and builders, as well as consultants, find it increasingly burdensome to implement standard STC (ASTM) field testing because of costs and delays. Inspectors are reluctant to rexuire testing because of the costs and delays, yet there seems to be no easy alternative available. This paper discusses the results of over 100 tests conducted in the Southern California area of party walls with the object of considering whether a scrcening method for party-wall checks can be used as a viable altemative to the ASTM method in field inspections. The paper will propose a possible technique for adoption as at supplement to ASTM methods currently employed.

\title{
Session E. Speech Communication I: Automatic Speech Understanding
}

\author{
June E. Shoup-Hummel, Chairperson \\ Speech Communications Research Laboratory, Inc., Santa Barbara, California 93109
}

Contributed Papers

$$
\text { 9:00 }
$$

E1. Revieu of the ARPA speech understanding project. Dennis H. Klatt (Room 36-523, Massachusetts Institute of Technology, Cambridge, MA 02139)

After five years of research and development, the final three speech understanding systems funded by the Advanced Research Projects Agency of the Department of Defense were demonstrated in early September of this year. As a member of the ARPA Steering Committee and as a consultant for one of the research groups, I will offer a summary of the capabilities that were demonstrated, and also speculate on the seientific knowledge gained during the course of the program. The opinions to be expressed are entirely the author's.
9:10

E2. The Harpy Speech Recognition Sy stem: performance with large vocabularies. B. Lowerre and R. Reddy (Department of Computer Science, Carnegie-Mellon University, Pittsburgh, PA 15213)

The Harpy System [B. Lowerre and R. Reddy, Harpy, a connected speech recognition system, J. Acoust. Soc. Am. 59, S 97 (A) (1976)] has been extended to run with large vocabularies. The system was recently tested with a 1011-word yocabulary language permitting about $10^{12}$ possible sentences for an Information Retrieval task using a computerized data base. The system achieved $93.77 \%$ word recognition accuracy $(89.8 \%$ sentence recognition accuracy) on 284 connected speech sen- 
tences (containing 1580 words) of about 3-sec duration each for a single speaker. The system requires about $12 \mathrm{Mipss}$ (million instructions per second of speech) and uses about 200000 words of memory on a PDP-10 system. More complete results, including several speakers and additional sentences, will be reported. [Research supported by the Defense Advanced Research Projects Agency. I

\section{$9: 20$}

E3. The Hearsay-II speech understanding system. L. 1). Erman, F. Hayes-Roth, V.R. Lesser, and R. Reddy (Department of Computer Science, Carnegie-Mellon University, Pittsburgh, PA 15213)

The Hearsay-II System has as its design goal recognition, understanding, and responding to connected speech utterances, particularly in situations where sentences cannot be guaranteed to agree with some predefined, restricted language model, as in the case of the Harpy System. Further, it at tempts to view knowledge sources as different and independent which eannot always be integrated into single representation. It is based on the blackboard model [V.R. Lesser, R. D. Fennell, L. D. Erman, and D. R. Reddy. IEEE Trans. Acoust. Speech and Signal Process. ASSP-23, 11-23 (1975) with knowledge sources as a set of parallel processes which are activated asynchronously depending on data events. The system performs on the Information Retrieval task with accuracy comparable to that of the Harpy system, but runs about 2 to 20 times slower. More complete performance results will be reported. As we get closer to unrestricted vocabularies and nongrammaticality of spoken languages, it will be necessary to have systems which have the flexibility of Hearsay- $\Pi$ and the performance of Harpy. [Research supported by the Defense Advanced Research Projects Agency. I

\section{$9: 30$}

E4. Feature extraction, segmentation, and labeling in the Harpy and Hearsay-II systems, H.G. Goldberg and R. Reddy (Department of Computer Seience, Carnegie-Mellon University, Pittsburgh, PA 15213)

Goldberg [J. Acoust. Soc. Am. 59, S97(A) (1976)] has shown that uniform techniques for segmentation and labeling can provide the initial signal-to-symbol transformation for speech recognition systems with reasonable accuracy and efficiency. Furthermore, the choice of parametric representation was not found to be critical for most commonly accepted representations. However, for efficiency, the computationally simplest techniques should be used to segment the utterance before more accurate (and expensive) spectral representations are used for labeling [R. Reddy, J. Acoust. Soc. Am. 42, 329-47 (1967)]. To provide an initial symbolic input for both the Harpy and Hearsay- $\Pi$ systems, an hierarchical, featureextraction based segmenter, using the ZAPDASH parameters, has been developed. After segmentation, labeling is done by a modified LPC minimum distance [F. Itakura, IEEE Trans. ASSP-23, 67-72 (1975)]. Labeling proceeds by comparing the midpoint of each segment with stored templates (acquired by an iterative learning process from speaker-specific training corpus) and adjusted with weights according to features obtained from the segmenter. The use of the highly efficient segmentation procedures and parameters provides approximately a factor of 5 speedup over uniform techniques which were previously used with both Harpy and Hearsay-II [Research supported by the Defense Advanced Projects Agency.]

$$
\text { 9:40 }
$$

E5. Connected Digit Recognition using symbolic representation of pronunciation variability. G. Goodman, B. Lowerre, R. Reddy, and D. Scelza (Department of Computer Science, Carnegie-Mellon University, Pittsburgh, PA 15213)

Most connected speech recognition systems such as Harpy and Hearsay-II use some form of symbolic representation alternative pronunciations of the vocabulary whereas most isolated word recognition systems use word templates. In an attempt to compare relative performance of systems that use symbolic representations of words, the Harpy system was run on a connected digit task requiring the recognition of random three-digit sequences. Each of ten speakers (seven male and three female) spoke 30 training sentenzes and 100 test sentences over a period of two weeks in a computer terminal room environment (approximately $65 \mathrm{~dB} A$ ). Using speakerdependent phoneme templates, the word error rate over all the ten speakers was about $2 \%$. Using speaker-independent phoneme templates computed from the training data for all the speakers (male and female), the word error rate was about $8 \%$ for a test data set of 1200 random connected three-digit sequences from 20 speakers (including ten new speakers). The recognition time is about 4.5 Mipss (million instructions per second of speech). [Research supported by the Defense Advanced Research Projects Agency. 1

\section{$9: 50$}

E6. Parametric representation of speech. G. Gill and R. Reddy (Department of Computer Science, Carnegie-Mellon University, Pittsburgh, PA 15213)

As digital processing of speech beco;nes commonplace, it becomes desirable to have a parametric representation of speech which is simple, fast, accurate, and directly obtainable from the PCM representation of sjeech. The ZAPDASH representation of speech (Zerocrossings And Peaks of Differenced And Smooth waveforms) is one such. The PCM data is used to generate a different waveform and a down sampled, smoothed waveform (for $10-\mathrm{kHz}$ sampling rate, the smoothing FIR filter coefficients were $-1012444210-1$, used every fourth point). Peak-to-peak distances and number of zerocrossings are calculated each $10 \mathrm{msec}$, resulting in 400 8-bit parameters per second of speech. Z APDASH can be done in 15-20 computer instructions per sample and can be extracted in less than a $1 / 3$ real time on minicomputers with $2 \mu$ sec instruction time. Although this representation is not noticeably different other similar proposals, it seems to be fairly robust and accurate, and is used in the feature extraction, segmentation, and labeling parts of the Harpy and Hearsay- $\Pi$ systems. Fortran and PDP- 11 machine language versions are available from the authors. [Research supported by the Defense Advanced Research Projects Agency.]

\section{0:00}

E7. The HWIM speech understanding system-Overview and performance. Jared J. Wolf and William A. Woods (Bolt Beranek and Newman Inc., 50 Moulton St., Cambridge, MA 01238)

HWIM (for Hear What I Mean), the speech understanding system developed at BBN as part of the recent five-year ARPA Speech Understanding Research Project, is designed to "understand" naturally spoken utterances relevant to a task domain of travel budget management. Its vocabulary is over 1000 words, and its grammar permits $\varepsilon$. habitable subset of natural English. HWIM contains sources of knowledge at the levels of acoustic-phonetics, phonology, vocabulary, syntax, semantics, factual knowledge, and discourse. This paper describes the system as a whole and presents its performance results at the end of the ARPA project. [This research was supported by the Advanced Research Pr jjects Agency of the Department of Defense and was monitored by ONR under Contract No. N00014-75-C-0053.]

\section{0:10}

E8. Phonetic and lexical processing in the HWIM speech understanding system. Richard M. Schwartz, John W. Klovstad, Victor W. Zue, John I. Makhuul, and Jared J. Wolf (Bolt Beranek and Newman Inc., 50) Moulton St. , Cambridge, MA 02138)

The "front end" of HWIM, the BBN speech understanding system, is that part of the system that governs the formation 
and eraluation of hypotheses between the levels of the speech signal and the word. It comprises processes for signal processing, acoustic-phonetic recognition, Iexical-segmental matching, and lexical-parametric matching. Implicit in the lexical matching processes is the application of phonological rules, both within word pronunciations and across word boundaries. A consistent scoring policy gaverns the evaluation of hypothesis at the segmental and word levels, and this policy is carried into the control component of the system, where it is applied to multiword hypotheses about the interpretation of the utterance. [This research was supported by the Advanced Research Projects Agency of the Department of Defense and was monitored by ONR under Contract No. N00014-75-C-0053. I

\section{$10 \div 20$}

E9. Linguistic processing and control strategy in the HWIM speech understanding system. William $A$. Woods, Madeleine Bates, Geoffrey Brown, and Jared J. Wolf (Bolt Beranek and Newman Inc., 50 Moulton St., Cambridge, MA 02138)

The principal source of higher-level linguistic knowledge in HWIM, the BBN speech understanding system, is an augmented transition network parser, which embodies the syntactic, semantic, and part of the factual sources of knowledge of the system. It parses sentences or sentence fragments in either direction, and it can, for a sentence fragment, enumerate the words and syntactic/semantic classes permissible at the ends of the fragment. The control component of the system is a program that calls on the other sources of knowledge of the system in order to formulate, evaluate, and extend hypotheses about the interpretation of the utterance. It is responsible for guiding the system to the most likely interpretation as efficiently as possible. [This research was supported by the Advanced Research Projects Agency of the Department of Defense and was monitored by ONR under Contract No. N00014-75-C-0053.

\section{$10: 30$}

E10. Word verification in a speech understanding system. Craig C. Cook (Bolt Beranek and Newman, Inc., 50 Moulton St., Cambridge, MA 02138) and Dennis H. Klatt (Room 36-523, Massachusetts Institute of Technology, Cambridge, MA 02139)

Given a word whose presence has been hypothesized in an unknown utterance, one way of enhancing the confidence in that hypothesis is to generate a synthetic parameterization of the word and then match it against the equivalent parametric representation of the unknown utterance. We have implemented such an approach in the speech understanding system under development at Bolt Beranek and Newman, Inc. Given a word, a synthesis-by-rule program generates a representation in terms of linear prediction spectra, which are matched against similar spectra of the raw signal using a 13-pole linear prediction error metric in conjunction with a dynamic programming time-normalization algorithm. Some automatic talker normalization procedures have been implemented in the synthesis strategy. The performance of the verification component has been measured by obtaining the distribution of verification scores for all word hypotheses generated by the speech understanding system, and determining the scores for words that should be verified correctly versus those seores for false word hypotheses. ISupported by ARPA under Contract No. N00014-75-C-0053. ]

\section{$10: 40$}

E11. Use of intonational phrase boundaries to select syntactic hypotheses in a speech understanding system. Wayne A. Lea (Speech Communications Group, Sperry Univac DSD, St. Paul, MN 55165)

A procedure has been developed for using prosodically detected phrase boundaries to weight word and phrase hypotheses in the Bolt Beranek and Newman (BBN) SPEECHLIS speech understanding system, so that correct words and structural hypotheses will be proposed at earlier stages in parsing, and erroneous theories can be avoided. The statetransition ares of the augmented transition network grammar were specially marked if they were expected to be immediately preceded by intonationally deteeted phrase boundaries. The scores on words associated with the ares were increased if expected boundaries were detected, or decreased if expected boundaries were missing in the acoustic-prosodic datil. Fifteen BBN sentences were processed through a computer program that detected phrase boundaries at fall-rise valleys in fundamental frequency contours. Analysis of simple traces of the hypothesizing, testing, and constructing of syntactic structures by the SPEECHLIS system showed that prosodic adjustment of scores would increase the likelihood of correct words and phrases being selected before incorrect ones. These ideas are being refined and tested further, for implementation in the SPEECHLIS system.

\section{0:50}

E12. Evaluation of an automatic word recognition system over dialed-up telephone lines. A.E. Rosenberg and F. Itakura* (Acousties Research Department, Bell Laboratories, Murray Hill, NJ 07974)

An evaluation of an automatic word recognition system [F. Itakura, IEEE Trans. Acoust. Speech Signal Process. ASSP-23, 67-72 (1975)] has been carried out over dialed-up telephone lines using a laboratory computer on line. Thirteen speakers participated in the evaluation, calling up the system once a day over a five-month period. In each experimental session speakers were instructed by voice prompt to provide utterances of 12 words spoken in isolation. These words were randomly selected in each session from an 84-word vocabulary, 50 of whose entries are North American cities, designed to give the speaker access to airline flight time-table information. At each trial the speaker was informed whether his utterance was correctly recognized or not. There are two categories of error: an incorrect match or a rejection (no match). Speakers were requested to repeat words not recognized on the first attempt a second and, if required, a third time. The average number of trials per speaker over the entire experimental period was 840 . The median percentage of incorrect matches on the first attempt over the 13 speaker was $2.7 \%$ while the median percentage of rejections was $5.7 \%$. The median percentage of words still not recognized after 3 attempts was $1.5 \%$.

*Now at Electrical Communications Labs., Nippon Telephone and Telegraph Corp., Tokyo, Japan.

$$
\text { 11:00 }
$$

E13. Statistical decision approach to the recognition of connected digits. M. R. Sambur and L. R. Rabiner (Acoustics Research Department, Bell Laboratories, Murray Hill, NJJ

A connected digit recognition system that uses a statistical decision approach based on an expanded form of the principle of minimum residual error has been developed. The expanded distance measure includes the effects of analysis estimation error, the effects of coarticulation, and the effects of speaker variability. The recognition system has been tested on six speakers in a speaker dependent mode with recognition accuracies near $100 \%$. It has also been tested with ten new speakers in a speaker independent mode, with a digit recognition accuracy exceeding $95 \%$.

\section{1:10}

E14. Labeling speech events for acoustic and linguistic processing. R.J. Hanson* and L. L. Pfeifer (Speech Communications Research Laboratory, Inc., 800A Miramonte Drive, Santa Barbara, CA 93109)

In speech studies, data base access has been significantly improved through the use of label files which relate portions of the speech waveform with a phonetic (or phonemic) transcription as well as other linguistic and nonlinguistic information. In this way the acoustic and linguistic "realities" of speech can be associated and checked against each other since they were derived independently. Because of the lack of a simple correspondence between the acoustic structure of a portion 
of the speech waveform and the linguistic label assigned, additional principles must be invoked to make labels useful. As an example of the labeling process and the accompanying problems, a sound identification study is described in which 675 vowel nuclei were labeled using the transcription derived from a sophist icated multitranseriber system. Despite precautions taken to insure correct labeling, many of the vowels are judged in a pattern recognition experiment to be more similar to neighboring vowels in the vouel space or to schwa than the vowel they were labeled as. These supposedly misidentified vowels were found to have acoustic structures very different from the ideal for their label, at least partially due to the loss of coarticulatory and vowel reduction information when assigning transeription symbols. Directions for resolving these problems and for increasing the utility of labeled speech events in acoustic and linguistic processing are discussed. [Work supported by AFOSR Contract No. 44620-74C-0034. I

$$
11: 20
$$

E15. Abstract withdrawn.

$$
11: 40
$$

E17. An algorithm for speaker verification. M. Shridhar and M. Vidalon (Electrical Engineering Department, University of Windsor, Windsor, Ontario N9B 3F4, Canada?

An analysis of the spectral properties of male speech reveals that most of the spectral energy is distributed in the frequency range of $50-2000 \mathrm{~Hz}$. The authors attempted to develop a computer algorithm for speaker verification that utilized parameters extracted directly from the digitized speech. The original speech signal was sampled at $4 \mathrm{kHz}$ after filtering it with a $2-\mathrm{kHz}$ low-pass filter. The investigations reveal that by the use of low-order linear predictor model a feasible set of parameters could be realized for application to speaker verification. A simple warping procedure modifies the parameter contour of the unknown speiker so that the correlation with the reference contour is maximize. The verification decision was based on the distance of the test sample contour from the reference contour for: the claimed speaker. If the distance was less than a fixed threshold, the speaker was accepted. Among all the parameters investigated, the reflection coefficients and the autocorrelation coefficients were found to be the most effective, providing a verification accuracy of $98 \%$ for speech, 2 sec in duration, which increased to more than $98 \%$ for a duration of 3 sec. In conclusion, a procedure for speaker verification has been developed and it is fairly simple, reasonably fast, and reliably accurate.

$$
11: 50
$$

E18. Long-term feature averaging in voice authentication. J. D. Markel, B. T. Oshika, and A.H. Gray, Jr. (Speech Communications Research Laboratory, $800 \mathrm{~A}$ Miramonte Dr. , Santa Barbara, CA 93109)

The purpose of this paper is to investigate the applicability of long-term feature averaging as an eventual means for performing text independent voice auttentication (speaker ver ification). Based upon a set of long-term feature vectors, a principal component analysis is performed to obtain a normalized reference coordinate system for each speaker. Features extracted from the test speaker are transformed to this coordinate system and then the Euclidean distance is measured. It is shown thatunder a weak assumption of Gaussian statistics, the threshold necessary to attain a given probability of correct acceptance as a function of the number of dimensions or features can be theoretically ealculated. Results of several preliminary experiments are presented to illustrate the technique. 


\title{
Session F. Shock and Vibration I: Vibration Signature Monitoring I: Diagnostics
}

\author{
Ronald L. Eshleman, Chairperson
}

Vibration Institute, Clarendon Hills, Illinois 60514

\section{Invited Papers}

9:00

F1. Diagnostics of machinery by acoustic source location. W. W. Schulz (Corporate Research and Development, General Electric Company, Schenectady, NY 12:345)

Signature Analysis has long been used to detect faults in a wide variety of machines. Recent efforts to locate faults by acoustic methods is illustrated by the location of corona in large power transformers. Excessive corona can damage insulation and require costly shutdown and repair. Corona generates both high-velocity electrical signals and ultrasonic signals which travel at the velocity of sound in the insulating oil. The acoustic signals are sensed by accelerometers attached to the exterior of the transformer tank. By triggering a signal average from the electrical signals, measurements of the acoustic travel time from the corona source to several transducers can be obtained. In simple cases these can be transformed to distance, and triangulation calculations yield a corona source location, More generally, the acoustic waves encounter obstacles, are reflected, and the triangulation techniques fail. A new technique called Space Interrogation systematically assumes a series of allowed corona locations and calculates the mean-square fit of the data to each assumed source location and set of permitted acoustic paths. The result is a more powerful means of locating corona sources.

\section{$9: 30$}

F2. High-frequency acoustic signals for condition monitoring. M. Darlow, O. Shinaishin, and L. Winn (Mechanical Technology, Inc., 968 Albany-Shaker Road, Latham, NY 12110)

Acoustic signals, structure borne, have long been used to detect the integrity of various machinery. This presentation discusses the state of the art of using the ultrasound (high frequencies beyond the audible range) of machine elements and structures to detect failure or malfunction conditions. The paper will describe a recently developed technique, the HighFrequency Resonance Technique (HFRT), its principles, and results of applications in detecting rotating machine element failure especially in bearing inspection, as well as its potential for extensive machinery condition monitoring. A brief discussion of the use of fiber opties in the monitoring of vibrations and detection of flaws in rolling element bearing will be presented. This relatively new technique shows promise for fault detection in the areas of inspection and vibration measurement in general. The paper will also discuss the use of high frequency signal detection of pulses generated in structures failing under dynamic loads and the potential of the method for nondestructive testing of various structures in important applications.

$$
10: 00
$$

F3. Applications of shock pulse technology to rolling element bearing condition monitoring. P. L. Howard (SKF Industries, Inc. , 1100 First Ave., King of Prussia, PA 19406)

The Shock Pulse technique has been widely and successfully applied. Recent testing indicates its capability to determine bearing condition and lubrication condition in the presence of background machine noise. Examples of applications to helicopter gear box bearing monitoring will be presented as well as current data on other applications ranging from Railroad Car bearing to rocket engine turbopump bearing monitoring.

$10: 30$

F4. Setting vibration limits from shaft amplitude measurements. D. S. Wilson (Manager of Engineering Services, Shaker Research Corporation, Ballston Lake, NY 12019)

Confusion often exists on setting vibrational amplitude limits for rotating machinery. Present day monitoring instrumentation often includes shaft-displacement-type sensors. Specifications for acceptable amplitude limits for vibration are more commonly available for bearing cap or housing vibration and do not reflect levels for shaft amplitudes. An approach is proposed to compute acceptable amplitude levels due to rotating unbalance to protect the rotating components. The approach may be extended to nonsynchronous motions of the rotating shaft. 
$11: 00$

F5. Development of an on-hoard seismic derailment detector. William W. Wassmann 'The Naval Surface Weapons Center/White Oak Laboratory, Silver Spring, MD 20910)

The Naval Surface Weapons Center/White Oak Laboratory, after conducting a feasibility study in 1972/73, has embarked on a two year program for the Federal Railroad Admi instration's Office of Research and Development to develop an on train system for train accident reduction (STAR). It will be installed for test on a 124-car unit ore train of the Duluth, Missabe and Iron Range (DM\&IR) Railway Company. The system includes a seismic wheelon-the-ground detector which senses an abnormal vertical motion of the unsprung masis of a freight car. The sensor then initiates a sequence of events which causes the train to stop. This paper describes the development of the seismic sensor : the initial feasibility study, establishment of design criteria, fahrication, installation, and evaluation of the sensor's. Initial calculations of a freight car wheel-drop trajectory suggest that there may be a siufficient velocity change above an acceleration threshold to make a discriminating spring-mass system feasible. Actual full-scale derailment tests and over-the-road vibration measurements support this theory. Sensors have been built and tested during actual freight train derailments and are currently in regular service on the DM\&IR between the loading plant and ore docks to obtain false-alarm data.

\title{
Session G. Psychological and Physiological Acoustics II: Electric Response Audiometry (ERA): Present Status and Problems
}

\author{
Special Session in Honor of Hallowell Davis
}

\author{
Robert Galambos, Chairperson \\ Department of Neurosciences, A012, University of California, \\ San Diego, La Jolla, California 92093
}

\begin{abstract}
It has been known for many years that sound stimuli can modify the electrical activity of the brain. The possibility of developing useful clinical tests of hearing based upon this fact has also Iong been recognized. Efforts to create such tests, however, have until receritly yielded relatively unimpressive results. The developments of the past five years are another matter, and this session will review them for interested psychologists and physiologists, and for specialists in instrumentation related to audiometry. New information on brain events time locked to stimulus presentation-the so-called evoked responses-will be emphasized, along with the recording methods presently used to visualize them. Speakers will describe the way these evoked potentials are being used in the testing of hard-ofhearing patients. By emphasizing certain limitations of the methods currently employed they will also point to the future developments by which electric response audiometry may become an even more powerful clinical tool.
\end{abstract}

\section{Panel Discussion}

$$
2: 00
$$

G1. Panel discussion. Hallowell Davis (Central Institute for the Deaf, St. Louis, MO 63110), Robert Galambos (University of California at San Diego, La Jolla, CA 92093), and Gecrge Moushegian (Callier Center for Communication Disorders, Dallas, TX 75234)

The Chairman will describe the auditory-evoked response as this is recorded through scalp electrodes from normal subjects in the laboratory. It consists of some 15 successive waves varying in latency from about $1.5-300 \mathrm{msec}$ poststimulus. Hallowell Davis will then present the principles of electric response audiometry and show how the so-called lat $\epsilon$ waves (latency $100 \mathrm{msec}$ and later) can be used to test hearing in adults and children. The way the early waves (latency $1.5-10 \mathrm{msec}$ ), which are generated by the auditory nerve and brainstem nuclei. aid diagnosis of hearing problems in infants and other difficult-to-test patients will be described by Galambos. Still another electrical event, the frequency following potential, which also is generated within the brainstem, will be dealt with by George Moushegian, who will provide a description of the phenomenon and show examples of its clinical utility. Following this review of the present status of ERA the panel will discuss its limitations. The question of whether hearing at frequencies below $2000 \mathrm{~Hz}$ is testable using the brainstem response will receive particular attention. 


\section{Contributed Papers}

\section{$3: 30$}

G2. Comparison of gross neural waveforms recorded from various electrode sites in the guinea pig. Curt Mitchell and Cynthia G. Fowler [Auditory Physiology Laboratory (Audiology), Northwestern University, Evanston, IL 60201]

Different way eforms of auditory evoked potentials were found when recording from locations in the cochlea, middle ear, and vertex of the skull in the guinea pig. The first negative potential $N_{1}$ maintained its latency and waveform from a variety of sites. However, the $N_{2}$ potential changed its peak latency and waveform considerably with location. At certain electrode sites, double-peaked $\mathrm{N}_{2}$ waveforms were found. The data suggest that the $N_{2}$ potential is a complex potential reflecting activity from more than one neural population. Thus, $N_{2}$ is composed of repetitive firing of cochlear afferents [Özdamar, $\mathrm{Ph}$. D. dissertation, Northwestern University (1976) ] and neurons in the cochlear nucleus or brainstem. The data do not : suggest the apical portion of the cochlea as an origin of $N_{2}$. The $N_{7}$ potential was found to be most prominent from the vertex; and $N_{1}, N_{2}$, and $N_{3}$ appeared at nearly the same amplitudes from certain positions. The potentials maintained their characteristic wave shapes over a range of intensities. [Supported by grants from the NINCDS.]

\section{$3: 45$}

G3. Auditory brainstem electric response to click trains and continuous tones. C.M. Suter, E. M. Glaser, A. Goldberg, and R. Dasheiff (Division of Otolaryngology, University of Maryland Hospital, Baltimore, MD 21201)

This study was undertaken to investigate the effects of click interstimulus interval (ISI) and sensation level (SL) on the short-latency auditory-evoked potential (AEP), and the relation of the AEP and the frequency-following response (FFR). Continuous tones of 250 and $500 \mathrm{~Hz}$ and $0.1-\mathrm{msec}$ acoustic clicks with ISI's of $250,70,17,8,4$, and $2 \mathrm{msec}$ were employed as stimuli at SL's of $70,50,30$, and $10 \mathrm{~dB}$. Both ISI and SL affect the AEP in a similar manner. A reduction in either results in an increase in response latency and a diminution of response amplitude. The relative effects are shown in terms of isolatency and isopotential curves for wave V. As the ISI becomes very short, the AEP becomes similar in waveform amplitude and threshold characteristics to the FFR to continuous tones.

$$
4: 00
$$

G4. Farfield recording of the frequency following response to the missing fundamental. J.T. Marsh, J.C. Smith, and W.S. Brown (Department of Psychiatry and Brain Research Institute, UCLA School of Medicine, Los Angeles, CA 90024)

Subjects were presented with a complex stimulus consisting of four tones: $730,1095,1460$, and $1825 \mathrm{~Hz}$ of equal intensity and cosine starting phase. For this stimulus $2 f_{1}-f_{2}=f_{2}-f_{1}$ $=365 \mathrm{~Hz}$. Subjects perceived the $365-\mathrm{Hz}$ combination tone and pitch matched it accurately to a pure tone at $365 \mathrm{~Hz}$. Vertex to earlobe farfield recordings of FFR were obtained for both the complex stimulus and the $365-\mathrm{Hz}$ pure tone. Both stimuli elicited FFR frequency of $365 \mathrm{~Hz}$. Spectral analysis showed FFR to the "missing fundamental" larger than response to pure tone. Narrow-band noise with $365 \mathrm{~Hz}$ center frequency masked the pure tone but not the missing fundaniental. Noise reduced the amplitude of FFR to pure tone by $8 \mathrm{~dB}$, but did not significantly affect FFR to the "missing fundamental,"

$$
4: 15
$$

G5. Bilateral brainstem responses to monotic click. S. Zerlin and R.F. Naunton (Section of Otolaryngology. Pritzker School of Medicine, University of Chicago, Chicago, IL 60637)

Brainstem-evoked responses (BER's) from both sides of the human head were simultaneously recorded by means of a vertex electrode with separate left and right earlobe references. Monotic clicks were presented via earphone at 75-dB sensation level and the BER's recorded bilaterally. Latencies of corresponding peaks of the BER from stimulated and unstimulated sides were compared, with these results: wave III, with latency of about $3.5 \mathrm{msec}$ was always somewhat delayed on the stimulated side; waves IV-VI, with latencies in the 4. 5-7.5-msec region, appeared simultaneously on the two sides. Studies on cat have associated wave III with the crossed neurons of the superior olivary complex and successive waves with other of the nuclei in the ascending auditory pathway. [J.S. Buchwald and C-M Huang, Science 189, (1975). I The present data support their finding that wave III is generated at the superior olivary complex, because of the latency effects manifested by wave III. [Work supported by NIH. ]

$4: 30$

G6. Brainstem auditory-evoked responses with amplification. Barbara Mokotoff, and Donald F. Krebs (Speech, Hearing, and Neurosensory Center, 8001 Frost Street, San Diego, CA 92123)

Brainstem auditory-evoked response (BER) measurements were obtained on adult volunteer subjects who were hearing aid users. The BER's were elicited with click stimulation in several conditions: (1) unaided via earphones, (2) unaided via speaker, and (3) aided via speaker. In the aided condition, subjects adjusted their own hearing aids to their usual comfortable listening level. Aided and unaided audiometric thresholds were also obtained on each subject using pure tones, narrow-band noise, and speech under phones, and using noise and speech in a sound field. Hearing aids were analyzed for frequency response and distortion with a series of inputs and also at user level. Comparisons were made between aided and unaided audiological and BER data. Results indicate that aided BER measurement compared favorably with aided audiological data on cooperative adults and may therefore become useful when applied to hearing aid evaluation procedures for infants and children.

\section{$4: 45$}

G7. Three-way ERA system simultaneously collects ECochG early and middle responses. R.J. Voots, L.A. Harker, and M. I. Mendel (Otolaryngology Research Laboratory, Medical Research Center, Room 4, Iowa City, IA 52242)

Laboratory investigations of electrical auditory responses are commonly categorized as early, middle, or late (latencies) and ECochG (electrocochleography). For clinical ERA with optimal use of patients' limited time, we have devised a three way combined system to collect, simultaneously on-line, data in three categories, independently, for a common stimulus set from a single array of electrodes. Two time-parallel early component channels are used, one for ECochG and one for brainstem responses (or optionally, both for brainstem responses from opposite sides of the head). A third channel collects middle responses. Conjunctly, we have also adopted a compound stimulus paradigm consisting of a click followed by a tone burst. This provides optrmal stimulation for basal turn responses (early and ECochG) while also yielding frequency specific data from the middle responses. It also appears to provide an unexpected bonus by counteracting the myogenic (sonomotor) response that sometimes contaminates the middle neurogenic response. Data from ten young normal adults will be reported.

\section{5:00}

G8. Brain response to intracranial auditory motion. H. B. Ruhm (Veterans Administration Hospital, San Diego, CA 92161)

Responses, recorded from scalp electrodes on human subjects were evoked by the onset of three types of modulation of 
a 100- $\mathrm{Hz}$ click train-AM, FM, and time difference modulation (TDM). The last consisted of intracranial sweeps generated by successive increments in the interau ral temporal differcnce between members of dichotic click pairs that composed the train. Slope and extent of TDM were independently varied. All types of modulation produced similar slow-wave responses. The direction of intracranial motion was reflected in the N1-P2 amplitude of the response to TDM in that, lateral-moving sweeps evoked larger response than medialmoving sweeps. Response latency decreased and amplitude increased with increasing TDM slope up to $5.55 \mathrm{msec} / \mathrm{sec}$ with little change tereafter. Amplitude also increased with increasing extent of sweep up to $0.55 \mathrm{msec}$. interau ral time difference. Extent of sweep did not systematically affect the latencies of N1 or P2. No right-left differences were observed. [Work supported by Veterans Administration. I

\title{
Session H. Underwater Acoustics II: Signal Processing and Noise. Precis Poster Session
}

\author{
James L. Stewart, Chairperson \\ Naval Undersea Center, San Diego, California 92132 \\ Contributed Papers
}

2:00

H1. Efficient computation of array patterns. Victor C. Anderson (Marine Physical Laboratory, Scripps Institution of Oce:mography, San Diego, CA 92132)

The impact of a symmetrical array geometry, the use of a quantized stored cosine function, the exploitation of Digital Fourier transform algorithms and the application of trigonometric interpolation in the computation of array patterns is discussed. Careful selection of parameters permits sampling the array pattern only $6 \%$ above the theoretical Nyquist limit. Reconstruction of a rray patterns showing $-20,-30$, and $-40-$ dB relative interpolation errors are presented. A saving of 8000: 1 in computation time over direct "brute force" array pattern computation is illustrated for a hypothetical array. [Research supported by the Office of Naval Research and the Advanced Research Projects Agency. I

\section{2:04}

H2. Design of transducer arrays with tapered side-lobe heights. Geoffrey L. Wilson (Applied Research Laboratory, The Pennsylvania State University, P.O. Box 30, State College, PA 16801)

Transducer arrays for many underwater sound applications have customarily been designed for equal sidelobe levels. This is, perhaps, as much due to the convenience of the analytical Dolph-Chebyshev technique as to any specific requirement. Using a simple adaptation of a previously reported numerical technique for symmetrical arrays [G. L. Wilson, J. Acoust. Soc. Am. 59, 195 (1976)], destgns can be obtained whose directional response has a taper on the envelope enclosing the side lobes. The method is equally applicable to sum and to difference patterns. Examples are given. [This work was sponsored by the Naval Sea Systems Command, Code SEA034. J

\section{2:08}

H3. Method for increasing the number of beams from a timedomain beamformer. J. H. Stockhausen and I. D. Walkty (Defence Research Establishment Atlantic, P.O. Box 1012, Dartmouth, Nova Scotia, Canada B2Y 327)

In a digital delay-and-add beamformer for a line array the number of beams that can be steered between broadside and endfire is equal to the travel time betvicen elements divided by the sampling interval. This number is independent of the number of elements and if the array is long there may be insufficient beams to cover the directional space completely. Without increasing the sampling rate, additional beams may be steered by interpolating between successive samples from every second element. In typical cises the degradation of performance is insignificant.

$$
2: 12
$$

H4. Cross-sensor beam forming with sparse arrays. H.P. Bucker (Naval Undersea Center, San Iviego, CA 921:32)

The quadrature field of a narrow-band signal measured at a sensor, or sensor group, in an array is a complex number that rotates with time. Thus time arerages of the quadrature field tend toward zero. However, elements of the cross-sensor field, i. e., the quadrature field at one sensor multiplied by the complex conjugate of the quadrature field at a second sensor does not rotate and can build up with time. By using the cross-sensor field, a beam pattern (for a one-wave, no-noise acoustic field) can be generated for a spare array which is the same as for a filled array with the same aperture. When real world effects, such as noise and multiwave acoustic fields, are considered the performance of the sparse array degrades more than the performance of the filled array. However, by time averaging the cross-sensor field the performance of sparse array is greatly improved. Calzulations for a 23-element sparse line array in a realistic simulated acoustic field show significantly improved performance when compared to a 23-element uniform array.

$$
2: 16
$$

H5. Nearfield Fourier transform imageng. Donald A. Murphy (Hughes Aircraft Company, Box 3310, Fullerton CA 92634)

The two-dimensional Fourier transform provides an efficient method of forming beams for a line array. The field as sampled by the evenly spaced array is the Fourier transform of the farfield source. Its resolution is limited by the receiving aperture and the signal-to-noise ratio it the array elements. When the field is stationary, the resoltion can be increased by integrating the Fourier transforms magnitude squared. If the field is also homogeneous the resolution can be increased by extending the aperture without adding additional elements 
by the method described by Nuttall el al. This method constructs the two-dimensional spectrum of objects in the farfield without grating lobes from the cross spectra of elements of the array. The array spacings must all be multiples of a basic spacing in order to make the calculation efficient. The method has been extended to apply to objects in the nearfield as well, and allows estimation of noise measured in the nearfield with sparce arrays with an efficient computer program.

\section{$2: 20$}

H6. Parametric receiving array beam patterns using a phase demodulator. J.K. Beard and J.J. Truchard (Applied Research Laboratories, The University of Texas at Austin, P. O. Box 8029, Austin, TX 78712)

In this paper, closed form solutions are derived for several configurations of the parametric receiving array with a phase demodulator receiver. The expression for parametric nonlinear acoustic phase modulation of a high-frequency spherical ware from a point source by a low-frequency plane wave is calculated. The result is in turn used to generate the solution for parametric receiving arrays with various pump transducers, including a truncated line source, a rectangular piston, and a circular piston. The solution of the parametric receiving array and a truncated line receiver is also found. A series of experiments was conducted with a 48 -ft parametric receiving array at a pump frequency of $90 \mathrm{kHz}$. These oxperiments duplicated several of the geometries used to obtain the theoretical results. Good agreement between theory and experiment was achieved. [Work supported by ONR]

H7. Signal-to-noise ratio optimization in hydrophone-preamplifier systems. J.W. Young (AMETEK, Straza Division,

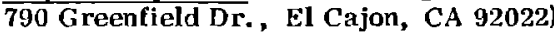

The decrease in signal-to-noise ratio caused by electronic noise is an important factor in the design of an acoustic receiving system. Normally, this loss is dominated by the hydrophone, cable, and preamplifier which make up the "frontend" of the system. In this paper, the signal-to-noise degradation factor (SND) for these components is defined. It is shown that the SND can be minimized by proper impedance matching of components. This can be accomplished either by use of a transformer or by the choice of a particular interconnection scheme for a multielement hydrophone. The minimum value of SND which can be achieved is determined by the product of figures of merit (FOM) of the hydrophone and preamplifier. For piezoelectric hydrophones, the FOM is a function of fundamental material parameters and the volume of the ceramic element. The amplifier FOM depends on the equivalent voltage and current noise sources of the device. Both charge amplifier and voltage amplifier configurations have been considered. The charge amplifier is found to have inherently poorer noise performance.

\section{$2: 28$}

H8. Programmable system for real-time data analysis and display. J. L. Bardin, V.M. Moore, and D.K. Raley (Applied Research Laboratories, The University of Texas at Austin, P.O. Box 8029, Austin, TX 78712)

A computer-based system for real time analysis and display of acoustic data is described. A mini computer and special purpose Fourier transform hardware are combined to permit continuous FFT and auto spectral analysis, and/or coherency analysis. In addition, software is available to perform various post-FFT analyses, including spectral whitening, automatic narrow-band energy detection and harmonic analysis. A second computer has been dedicated to the formatting and displaying of analysis-results. Being programmable allows operation of the computer-controlled display in various modes, such as A scan, time-frequency-amplitude alphanumeric, and combinations thereof. High-speed rotational engine radiated noise and line component data have been analyzed and results are presented.
H9. Spectral estimation techniques with special reference to sonar signal processing. A. Arcese and L. E. Bergeron (GTE Sylvania, Electronics Systems Group, Needham, MA 02194)

In this talk we present a comparative evaluation of several spectral estimation techniques. The estimation procedures investigated are the FFT and autoregressive modeling of the time domain waveform. For the autoregressive modeling the estimation procedures considered are the Durbin recursion, Burg recursion, Kalman filtering, and a gradient technique. We also present a new maximum entropy gradient spectral estimation procedure. The results of applying these spectral estimators to real submarine target datia are presented.

$$
2: 36
$$

H10. Signal excess and detection probability of fluctuating sonar signals in noise. R.J. Urick (Tracor, Inc., Rockville, MD 20850)

Signal excess is defined as the excess or deficiency of signal level relative to that required for a detection probability of $50 \%$; curves of detection probability versus signal excess are sometimes called "transition curves." In this paper, transition curves for a nonfluctuating signal in Gaussian noise and for Gaussian, Rayleigh, and log-normal signal fluctuations are obtained. A comparison with one set of detection data obtained in a sea test with a towed sound source indicates that log-normal signal excess fluctuations with a standard deviation of $6-8 \mathrm{~dB}$ give a reasonable fit to the data. The method suggested here-of using transition curves along with a known or assumed relation between transmission loss versus range-permits quicker computation of detection probability against range than does the conventional way of employing ROC curves, and can accommodate a signal excess that fluctuates with time about a mean ralue. [Work supported by Naval Surface Weapons Center and ASW Systems Project Office, Code ASW-13.]

\section{$2: 40$}

H11. Generalized performance. W.S. Hodgkiss (Naval Undersea Center, San Diego, CA 92132)

The performance of a Bayes optimal detector is summarized by its ROC curve. In the general case where uncertain parameters exist under each hypothesis, knowledge about them is explicitly noted at the outset by an a priori probability density function conditional to $\mathrm{H}_{1}$ and one conditional to $\mathrm{H}_{0}$. The processor's performance then becomes a function of their detailed shape. Often, the functional form of these densities is chosen so that various levels of uncertainty are easily modeled and a family of ROC's is reported. The question then arises: What performance would have been achieved under different prior knowledge assumptions (particularly when outside the class of densities modeled)? Or, more deeply: Does some algorithm exist which will operate on a known ROC for a given pair of priors to yield the ROC for a new set of priors? And, if not: Does a canonical intermediate step exist between observation and likelihood ratio statistics which always may be used as a starting point for the calculation of an ROC based on an arbitrary pair of priors? The intent of this paper is to pursue these questions. The discussion will uge as a basis the fundamental concepts of sufficient statistics and reproducing densities. A specific example is presented. [Work supported by ONR. $]$

$$
2: 44
$$

H12. Maximization of reverberation gain. D. Lee (Naval Underwater Systems Center, New London Laboratory, New London, CT 06320)

The problem of maximizing array gain (AG) for situations where ambient noise is the dominant interference has already been extensively investigated. These investigations have resulted in mathematical solutions having significant promise for various practical applications. A closely related and more 
general problem arises when interference is dominated by reverberation; in this situation the gain against reverberation (the reverberation gain, RG) must be maximized. The same general mathematical approach can be applied to both problems in that the omnidirectional ambient noise limited condition can be considered a specific case of the more general directional ambient noise limited condition and the reverberation limited condition. Much of the existing theory has been directed at the specific cases thus leaving unfinished a comprehensive treatment of the general case. In this presentation, the complete RG-maximization problem will be addressed; a mathematical procedure to achieve the solution will be outlined; this procedure is developed with sufficient generality so that the maximization of $A G$ is automatically included. An application of this technique is carried out for a three-dimensional array which demonstrates the validity and the effectiveness of this technique. [Work supported by NUSC.]

\section{$2: 48$}

H13. Who needs the octave-or, are standard bands feasible? L. C. Maples (Naval Underwater Systems Center, New London Laboratory, New London, CT 06320)

Although with the advent of the fast Fourier transform and digital filtering the constraints of available analog filter specifications are no longer really pertinent, the tendency to use the ANSI standard frequency bands for reference and comparison is widespread, particularly the so-called $\frac{1}{3}$-octave bands. However, in problems involving complex spectra with shifting narrow-band components, it becomea obvious that the $\frac{1}{3}$-octave approach is not adequate and may give misleading results, particularly at low frequencies, where the bandwidth is narrow. Similarly, because of their conceptual nature and also the way in which the bands are specified, consistent subdivision into narrow proportional bands is not possible, and such subdivision is now desirable, if not essential. For this reason, a return to the decimal system, in name as well as in fact, is strongly recommended. The problems encountered with standard " $\frac{1}{3}$-octave" bands are discussed in detail, in the context of the band specifications, and alternative methods of band specification, which avoid the problems entirely, are described, including a new look at the concept of "spectrum level."' [Work supported by NUSC.]

\section{$2: 52$}

H14. Interfrequency correlations of ocean ambient noise. R.H. Nichols and C.E. Sayer (Bell Laboratories, Whippany, NJ 07981)

The correlation of ambient noise levels in each of several frequency bands with those in other bands was investigated for an 1 - $\frac{1}{2}$-day period in February with a hydrophone laid on the ocean bottom in deep water. The bandwidth for the measurements was $6 \mathrm{~Hz}$; the center frequencies were chosen to cover the low frequency range in which shipping is generally the dominant source of noise, $10-150 \mathrm{~Hz}$ [G. M. Wenz, J. Acoust. Soc. Am. 34, 1936-1956 (1962)]. A total of 1562 consecutive 640-sec samples was taken in each frequency band; the average level for each sample was calculated and used in the computation of the correlation coefficients. Tonal line components were excluded. It was found that the coefficients of correlation between sample levels at a given frequency $f_{0}$ and those at other frequencies generally decreased with increased frequency spacing, but the patterns differed for different $f_{0}$ 's. Typical patterns will be shown. [Supported by IR\& D program.]

\section{2:56}

H15. Seasonal dependence of ambient sea noise near the marginal ice zone of the Greenland sea. James R. McGrath (Naval Research Laboratory, Washington, DC 20375)

Omnidirectional ambient sea-noise measurements in the 20$60-\mathrm{Hz}$ band near the marginal ice zone were recorded in the deepwater basin of the Greenland sea. This experiment, conducted between August 1972 and July 1973, provided 1-min samples of sea noise every $4 \mathrm{~h}$ using an omnidirectional hy- drophone $306 \mathrm{~m}$ below the surface. During this time, the ice edge moved over the recording site, providing evidence that the iceline acts as a noise souree, raising local levels above both open-ocean and ice-field values. Seasonal conditions favor the lowest ambient noise levels turing midwinter (January) and early summer (June), while the highest levels occur during early spring (March) and early fall (November). Evidence indicates that the maximum ambient noise levels near the marginal ice zone are $12-16 \mathrm{~dB}$ higher than the maximum Arctic Ocean values under contiguous ice cover.

\section{3:00}

H16. Measurements of directional ambient noise. S.O. McConnell (Applied Physics Laboratory, University of Washington, 1013 N. E. 40th St., Seattle, WA 98185)

Measurements of the directional ambient noise field havo been made in a bay using an acoustic lens for which the beam pattern closely approximates a circulc.r piston for the individual transducers $(D I=28.7$ at $25 \mathrm{kJI})$. The intensity radiation pattern of the noise at frequencies between 5 and $40 \mathrm{kHz}$ is found and compared to models of the form $\sin ^{2 n} \theta$ where $\theta$ is the grazing angle and $n=1,2,3$. This form for the radiation pattern is used for comparison since frevious measurements made at frequencies below $5 \mathrm{kHz}$ can ke fitted by a $\sin ^{2} \theta$ pattern at frequencies above $\sim 500 \mathrm{~Hz}$. Since the ambient noise at these frequencies appears to be generated at or near the sea surface due to such mechanisms as splashing of droplets, the intensity of the noise should be a function of conditions at the sea surface; a supposition borne out by these and previous measurements. Measurements are made of the wind shear and one-dimensional wave spectrum and rolated to the intensity of the noise. Measurements of the rate of rainfall and observations of intermittent noise sources are also made.

\section{3:04}

H17. Noise generated by axisymmetric turbulent boundary layer flow. G. C. Lauchle (Applied Research Laboratory, The Pennsylvania State University, P.O. Fox 30, State College, PA 16801)

The noise generated by hydrodynamic flow over an axisymmetric body with a blunt nose is deser bed quantitatively. Flush-mounted piezoceramic hydrophones were used to measure that part of the turbulent boundary-layer pressure fluctuations that propagates as true sound. Power spectra of the sound pressure were measured in a $3-50-\mathrm{kHz}$ frequency range over a wide range of Reynolds number ${ }^{3}\left(U_{\infty} D / \nu \leq 3.03 \times 10^{6}\right.$, where $D$ is the diameter of the body) for the model operating in the Garfield Thomas Water Tunnel. The use of flush-mounted hot-film probes to locate turbulence transition is also described. The power spectra of the noise measured in the laminar flow regions correspond closely to those measured in the transition and fully developed turbulent regions of the flow. The exceptions were those spectra measured on the flat part of the nose, but correction for diffraction loss effects suggests that the noise measured there is due to the noise generated by the turbulent part of the flow. Nondimensionalization of the noise spectra measured at various arc lengths with theoretical expressions for the noise expected, that include a critical boundary layer thickness at the beginning of turbulence, show a general collapse of data to within 6 dB. When applicable. comparisons of the radiated noise measured on buoyant bodies are made and agreement was found to he excellent.

\section{$3: 08$}

H18. Infrasonic flow-noise measurements using an H-58 hydrophone. O.M. Griffin and J.R. MoGrath (Naval Research Laboratory. Washington, DC 20375) and R. A. Finger (CBS Laboratories, Stamford, CT 06905)

Infrasonic and low-frequency flow-noise measurements were made during laboratory tests of an H-58 omnidirectional hydrophone. The hydrophone was tested in three configurations: bare, framed, and faired. Water speeds were varied from 0.25 to 0.45 knots and corresponded to a Reynolds number 
range from 8800 to 16000 . For different hydrodynamic reasons, the faired and framed configurations developed flownoise levels lower than the bare hydrophone configuration. The bare hydrophone developed the highest noise levels at the lowest frequencies of the $1-50-\mathrm{Hz}$ band. Hydrodynamic considerations related to the acoustic measurements made during this test are discussed; the importance of hydrophone configuration used in very low-frequency measurements is demonstrated.

\section{$3: 12$}

H19. Noise from cavitating hydrofoils as influenced by boundary-layer development. William K. Blake and F. Ellsworth Geib, Jr. (David W. Taylor Naval Ship Research and Development Center, Bethesda, MD 20084)

This paper describes measurements of noise from cavitating flow over a hydrofoil. Cavitation was generated on a hydrofoil in the presence of a separated laminar boundary layer on the one hand and of a fully turbulent attached boundary layer on the other. The turbulent boundary layer was formed downstream of a trip which was positioned near the leading edge. The noise is shown to depend on the type of cavitation produced. Dimensionless spectral densities of the sound are shown for each type of flow. For the cavitation associated with turbulent boundary layer development, the dimensionless spectrum is interpreted in terms of the observed single-bubble motions in the experiment.

\section{$3: 16$}

H20. Confidence limits for measured supertanker-radiated noise levels. J. Cybulski and E. B. Wright (Code 8160, Naval Research Laboratory, Washington, DC 20375)

Sound pressure spectral levels were obtained for the supertanker class of ships using a developing technique of aircraft deployed sonobuoys with a deep hydrophone at $305-\mathrm{m}$ depth. These measurements were made in the deep oceanic waters over the Seine, Tagus, Iberian and Biscay abyssal plains. The received levels at individual omnidirectional sensors were processed to yield ship radiated noise in $0.2-\mathrm{Hz}$ bandwidths over the $5-80-\mathrm{Hz}$ band and transformed to sound pressure levels in $\mathrm{dB} \| \mu \mathrm{Pa}$ at $1 \mathrm{~m}$. Confidence limits that are constructed for these values reflect the effects of navigation, calibration, geometry reconstruction. CPA determination and a transmission loss correction associated with the range between ship and sonobuoy sensor. The variation of these limits is illustrated as a function of CPA, aspect angle and frequency with standard errors as high as $3 \mathrm{~dB}$. The primary sources of error are hydrophone/system calibration, CPA determination and the transmission loss values. The FACT acoustic-environmental model was employed to obtain the frequency-range dependence corrections. Directions toward increasing the confidence of these measurements are indicated. [Work sponsored by Naval Electronics Systems Command. Code 320.]

\section{$3: 20$}

H21. Ambient sea noise directionality: Measurement and processing. N. Yen (Naval Underwater Systems Center, New London Laboratory, New London, CT 06320)

The response of a receiving hydrophone array in a noise field is analyzed for the relationship between the array parameters and the directionality of the noise field. The base functions formulated by array element spacing are considered as the coordinates of a multidimensional space. By orthonormalization of these base functions, the cross-spectra between hydrophone outputs are transformed directly to noise field directionality. Applying the same method to the array's beamforming output also gives another noise field estimate. When the number of independent measurements from the array's beamforming output is the same as that of the cross spectrum, the noise fields estimated by either methods are equivalent and depend on the array's structure. For stationary noise fields with low variability in the measured data, a further improvement in the angular resolution of the noise field can be achieved by deriving a new set of orthogonal base functions through analytic continuation. Interferences caused by uncorrelated local noise together with limits in the precision of numerical computation are shown to impose a practical limit on the accuracy of the estimated noise field. Thie processing method for noise directionality is illustrated by application to a simulated noise field and sea data acquired from a vertical line array. [Work supported by NAVSEA.]

\section{$3: 24$}

H22. Project SPAN 3: Low-frequency ambient sea noise in the South Fiji Basin. R.W. Bannister, R. N. Denham, and K. M. Guthrie (Defence Scientific Establishment H.M.N. Z. Dockyard, Auckland 9, New Zealand) and D. G. Browning (Naval Underwater Systems Center, New London Laboratory, New London, CT 06320)

Measurements of low frequency ambient sea noise near the New Zealand coast show relatively low levels due to a generally sparse shipping density in the Southern Hemisphere. [R.W. Bannister et al., J. Acoust. Soc. Am. 55, 418 (1974)] Project SPAN 3 extends these measurements to a deep ocean basin, specifically the South Fiji Basin located to the north of New Zealand. Ambient noise $(10-500 \mathrm{~Hz})$ was recorded continuously for one week on five hydrophones which were suspended between 300 and $2300 \mathrm{~m}$ depth in $4000 \mathrm{~m}$ of water. Supporting propagation loss measurements and ship density surveys were conducted. The results are in agreement with the previously reported data. [Work supported by NUSC and DSE.] 


\title{
Session I. Physical Acoustics II: Acoustic Levitation
}

\author{
Robert E. Apfel, Chairperson \\ Department of Engineering and Applied Science, Yale University, \\ New Haven, Connecticut 06520
}

Invited Papers

$2: 00$

I1. Radiation pressure-the history of a mislabeled tensor. Robert T. Beyer (Department of Physics, Brown University, Providence, RI 02912)

The acoustic radiation pressure has found practical applieation in recent years in instruments measuring sound intensity and in experiments on acoustic levitation. A historical review of the concept would the refore seem appropriate. The history of light radiation pressure goes back more than 200 years, beginning with Leonhard Euler and including such worthies as Maxwell, Boltzmann, and Lebedev. The concept of acoustic raldiation pressure dates back some 100 Years, and its history features the names of Rayleigh, Poynting, Langevin, and Brillouin among many others. It was pointed out by Brillou in that what we call radiation pressure is not a pressure at all, but a diagonal tensor, all the diagonal terrus of which are not identical. The size of the effect is small and the values obtained for the radiation pressure are very sensitive to boundary conditions and to approximations. In addi:ion, although the phenomenon is one of nonlinear acoustics, it can be observed down to the lowest sound intensities under certain conditions. Thus, the Rayleigh radiation pressure vanishes for the linear case but the Langevin pressure doe not. It might be said that radiation pressure is a phenomenon that the observer thinks he understands-for short intervals and only every now and then. [Work supported in part by the Office of Naval Research.]

I2. Acoustic levitation furnace for the space lab mission. E. G. Lierke (Battelle-Institut E. V., 6 Frankfort/M90. Postfach 900160, West Germany)

Abstract not received.

$3: 05$

I3. Acoustic levitation and manipulation for space application. Taylor G. Wang (Jet Propulsion Laboratory, California Institute of Technology, 4800 Oak Grove Dr., Pasadena, CA 91103)

The acoustics in space, apart from the obvious function of communication, has branched out into many other areas of applications. The acoustical positioning device developed at JPL for manipulating and controlling liquid systems in a $0-g$ environment has the poteritial of becoming one of the most versatile tools for space research and applications. For example, in order to study the liquid drop systems originated from the Newton-Cassini controversy experimentally, we need a free suspended liquid drop without any dense medium surrounding it. Hence, both the $0-g$ environment and the acoustic manipulation chamber appear to be necessary. In order to carry out many of the processes for Material science Laboratory in Space the melt is to be positioned and formed within a container but without making contact with the container walls. Electromagnetic methods of positioning and forming are limited to melts which are electrically conducting. The acoustical method is useful. in the control of any moltem material including material that is electrically nonconducting. In this presentation, I will describe the principle of operation of this apparatus and the results of tests both in our laboratory and in the $0-g$ environment provided by the Johnson Space Center aircraft flights.

I4. Acoustic levitation-a useful experimental technique. L. A. Crum (Department of Physics, U.S. Naval Academy, Annapolis, MD 21402)

The nonzero, time-averaged acoustic force exerted by a sound field on a local inhomogeneity allows liquid, solid, and gaseous particles to be suspended in a fluid medium relatively free of physical restraints. This splendid isolation allows studies to be made of radiation pressure, rectified diffusion, damping constants, acoustic streaming, surface waves, and physical parameters of metastable states, as well as many other phenomena, that would be nearly impossible without it. A review will be given of the general technique, and of various ways it can be utilized to obtain experimental data. [Work supported by ONR.] 
TUESDAY, 16 NOVEMBER 1976

\title{
Session J. Noise II: Prediction and Reduction of Traffic Noise Sources
}

\author{
Glenn E. Warnaka, Chairperson \\ Lord Corporation, Erie, Pennsylvania 16412
}

Invited Papers

$2: 00$

\begin{abstract}
J1. Control of diffracted sound by means of thnadners. L.S. Wirt (Lockheed-California Company, Department 75-40, Building 229, Plant 2, P. O. Box 551, Burbank, CA 91520)

The diffraction of sound around barriers partially defeats efforts to control noise by interruption of line of sight. Recent legislation, which limits allowable highway noise in adjacent communities, has stimulated interest in this problem. Currently, solid walls are used to provide partial acoustical shadows. This approach is expensive, partly because of nonacoustical engineering requirements. A review of Fresnel diffraction theory suggests that either transparency gradients or phase velocity gradients or both may be used to provide deeper shadows. Elemental Fresnel theory is slightly modified to introduce transparency or phase gradients near the diffracting edge and parametric studies are reported which define configurations that deepen the shadows. Scale model tests are described which display the trends predicted by the theory. This agreement leads to the fascinating conclusion that the best way to improve the performance of a solid wall is to cut a substantial portion of it away. Structures are suggested for the practical implementation of the method into noise barriers. A literary analogy suggests that such structures be called Thnadners.
\end{abstract}

\section{Contributed Papers}

$2: 30$

J2. Preliminary account of some recent experiments on sound diffraction by barriers. Allan D. Pierce* (Max Planck Institut für Strömungsforschung, D 3400 Göttingen, Federal Republic of Germany) and Robin $A$. Vidimos and $W$. James Hadden, Jr. (Georgia Institute of Technology, Atlanta, GA 30332)

Experiments performed summer 1976 in an anechoic chamber at NASA Langley Research Center consisted of (1) diffraction around screen from point source not necessarily far from edge, (2) such diffraction when air is blowing parallel to screen sides with diffracting edge as trailing edge, (3) diffraction of noise from a jet of air exiting on one side of a thin screen, and (4) diffraction of sound around a barrier with curved top with radius of curvature larger or comparable to an acoustic wavewavelength. These experiments were prompted by problems of assessing effects on aircraft noise when engines are mounted above wings. Accompanying theoretical work consisted of (1) generation of a convenient rapidly convergent numerical integration method for computation of Green's function given arbitrary sources and listener locations near a rigid wedge and (2) extension of Candell's solution of plane wave diffraction by screen in presence of ambient air flow to the point source case. [Work supported by NASA Langley Research Center.] *A.D. Pierce currently on leave of absence from GIT and is supported by award from Alexander von Humboldt Foundation.

$$
2: 45
$$

J3. Stochastic modeling of community noise levels. J.C. Freytag and F.H. Brittain (Noise Control Program, Bechtel Scientific Development, P.O. Box. 3965, San Francisco, CA 94119)

A modeling technique is proposed for combining two or more noise histograms (i. e., noise level versus duration histories) into a new histogram. By considering the time varying nature of independent noise sources, much more accurate community noise measures may be computed. Logarithmic addition of statistical measures (e.g., $L_{e q}$ or $L_{50}$ ) invariably overestimates the true result. The method is particularly useful in modeling a multisource environment such as a construction site where the duty cycles of independently operating equipment are accurately known. The analytical procedure is presented with a construction noise example.

\section{$3: 00$}

J4. Truck noise emission reductions and/or roadside barriers; the effects of each on population impact from motor vehicle noise. Basil H. Manns (Environmental Protection Agency, Washington, D.C., 20460), and Richard J. Peppin (1711 Westwind Way, McLean, VA 22101)

The Revised Design Guide (NCHRP 3-7/3) noise prediction program was modified to include reduced source noise levels. Medium and heavy truck noise levels were reduced in steps of $2 \mathrm{~dB} A$. The sound levels for receivers in two scenarios were calculated using roadside barrier heights, which varied from $0-21-\mathrm{ft}$. These scenarios included a flat, open area and an area which included two rows of single-story houses. Population impact, varying source level, scenario type, and barrier height was evaluated using a modified form of the method in the Revised Design Guide. This modified method allows the sound level to vary linearly with the logarithm of distance instead of the stepwise variation that is used now. Results presented show the effect on "impacted" population by reducing emitted noise levels of trucks and erecting roadside barriers.

\section{$3: 15$}

J5. New vehicle certification measurement for automobiles and light trucks. Paul D. Schomer (University of Illinois, Department of Electrical Engineering, Urbana, IL 61801)

Current certification measurement procedures are designed to measure the maximum noise that a vehicle can produce. Studies by GM and others have shown that vehicles are rarely operated in their maximum noise producing mode. More typical are operations such as urban acceleration (normal acceleration from a stop light, etc.), cruise and idle. To provide more meaningful new vehicle certification procedure and standard 
for the State of Illinois, an urban acceleration measurement procedure has been tested and developed. The procedure is based upon methods proposed by Hillquist and can currently be applied only to vehicles having an automatic transmission. The procedure is simple to perform, fairly short in duration and the results are quite repeatable. This paper deals primarily with the experiments used to develop the procedure and the resulting recommended practice.

$$
3: 30
$$

J6. Development of a motorcycle exhaust system certification program. John B. Walsh (U.S. Suzuki Motor Corp. 13767 Freeway Dr., Santa Fe Springs, CA 90670), and Roger Hagie (Kawaski Motors Corp., U.S. A., 3630 Garry St., Santa Ana, CA 92704), and Robin T. Harrison (USDA Forest Service, Equipment Development Center, 444 East Bonita Ave., San Dimas, CA 91773)

Excessive noise from modified motorcycles annoys many citizens and has damaged the reputation of the motorcycle industry. Enforcement action against excessively noisy motorcycles is necessary, and an exhaust system certification program could be used as a tool to reduce motorcycle noise impact. The Motorcycle Industry Council, representing most motorcycle and motorcycle accessory manufacturers, recognized the need for control of noisy motorcycle exhaust systems, and initiated development of an exhaust system certification program for motorcycles. The program was to provide the maximum amount of noise control possible through an enforcement program of low to medium intensity. The hope is that the program will help remove the excessively noisy exhaust systems from the market with minium disruption to responsible motorcycle and after market manufacturers. The manufacturers are taking steps to provide quieter motorcyles and exhaust systems. By working with enforcement agencies concerned with effectively controlling motorcycle noise, the industry hopes to get noisey bikes off of the streets and to finally reduce adverse noise impact from motorcycles.

$$
3: 45
$$

J7. Interior and exterior diesel locomotive noise measurements. Angelo J. Campanella (ACCULAB, . 3201 Ridgewood Dr., Columbus, OH 43220)

Diesel locomotive noise data accumulated over the past two years for hearing-damage assessment and land-use analysis are presented. Interior locomotive noise levels of the cab and engine areas were measured while the engine was developing all power levels was measured. Cab noise levels varied from 88 to $91 \mathrm{~dB} A$ at full power, depending on engine type, when the windows and doors were closed. Engine areas noise was found to be between 104 and $124 \mathrm{~dB} A$ at full power, depending on engine enclosure design. Extenal noise emissions were measured along an active east-west pair of tracks to determine land use classification for residences. This site typified enroute noise emission on flat agricultural terrain. Numerous observations were plotted versus distance from the tracks to derive an enroute emission model. Land use disposition was determined for future residences.

$$
4: 00
$$

J8. Ground-borne noise and vibration study - Toronto Transit Commission Yonge Street Northern Extension. Hugh J. Saurenman (Wilson, Ihrig \& Associates, Inc., 5605 Ocean View Dr., P.O. Box 2900, Oakland, CA 94618)

Following construction of the Toronto Transit Commission Yonge Subway Northern Extension (YSNE), train operations created ground-borne noise and vibration which seemed unusually noticeable in buildings near the tunnel. This paper will briefly summarize some of the conclusions of an extensive study and measurement program that was undertaken to evaluate both the characteristics of the ground-borne vibration and various procedures for reduction of the vibration and noise. The measurements included tests intended to:
(1) determine the characteristics of the ground-borne vibration and noise from the transit trains operating in YSNE tunnels, and (2) evaluate the effectiveness of design features and modifications to the facilities and transit cars in minimizing and reducing the noise and vibration. Some of the specific methods of controlling grouncl vibration include; use of resilient wheels, reduction of the rail fastener elastomer pad stiffness to decrease the coupling between the rail and the subway invert, grinding the rail, and turning the wheels. The vibration was measured at the tunnel invert. ground surface, and the walls and floors of adjacent buildings. In addition the interior noise induced by the ground-korne vibration was measured and analyzed.

\section{$4: 15$}

J9. Noise control of the Standard Light Rail Vehicle. R.H. Spencer (M/S P50-04, The Boeing Vertol Company, P. O. Box 16858, Philadelphia, PA 19142)

The Standard Light Rail Vehicle (SI.RV), currently being produced in quantity for Boston and San Francisco, has been designed to stringent interior and wayside noise criteria. The articulated joint in the carbody required an unusual design to reduce acoustic flanking paths. The average carbody transmission loss exceeded $30 \mathrm{~dB} A$ for the roof and sidewalls and was $35 \mathrm{~dB} A$ for the floor. The carbody design resulted in an interior noise level of $75 \mathrm{~dB} A$ at $40 \mathrm{mph}$, whether operating at grade or underground. Equipment roise levels were generally below $65 \mathrm{~dB} A$ at a distance of $15 \mathrm{ft}$ from the track centerline. Acoustically damped wheels eliminated squeal on curves and resulted in 40-mph pass-by noise levels of $70 \mathrm{~dB} A$ at a distance of $50 \mathrm{ft}$ from track centerline. Considerable appeal is expected by transit patrons, when the cars are introduced into revenue service.

$$
4: 30
$$

J10. Interior noise measurements on the MBTA Green Line. Steven L. Wolfe (Wilson, Ihrig \& Assciates, Inc. , 5605 Ocean View Dr., Oakland, CA 94618)

Measurements were made inside a PCC car with steel wheels, a PCC car with Acousta-Flex wheels and a new Standard Light Rail Vehicle (SLRV) simulating a typical revenue trip over the entire Massachusetts Bay Transit Authority Green Line from Riverside to Lechmore Stations and return. The results indicated peak noise levels from wheel squeal of $106 \mathrm{~dB} A$ and an average noise level of: $82 \mathrm{~dB} A$ in the steelwheel PCC car. These levels were reduced 10 and $4 \mathrm{dBA}$, respectively, in the $\mathrm{PCC}$ car with Acousta-Flex wheels; and 20 and $10 \mathrm{~dB} A$, respectively, in the SLRV. Statistical distribution charts of the entire runs are puesented along with $\frac{1}{3}-$ octave band analyses of selected events showing the relative levels and predominant frequencies of wheel squeal, general running noise, and stationary noise.

\section{$4: 45$}

J11. Effect of traffic mix volume and speed on highway noise levels. Richard J. Peppin (1711 Westwind Way, McLean, VA 22101) and Basil H. Manns (Environmental Protection Agency, Washington, DC 20460)

Since Johnson and Saunders ["The Evaluation of Noise from Freely Flowing Traffic," J. Sound Vij. 7, 287-309 (1968)] derived an expression relating the median traffic noise level as a function of traffic flow characteristics, many other relationships have emerged. These relationships have been used to design nomographs and computer noise prediction programs. This paper discusses the various basic formulation equations used to relate traffic mix and speed to the noise levels from motor vehicles. Parametric analysis of the basic equations used in the Revised Design Guide (NCHRP Report 3-7/3) for automobiles, medium trucks and heavy trucks, is performed. The effects of variations of traffic mix volume and speed on "equivalent" noise levels at receivers are illustrated. Figures are plotted so that the results can be used to find the minimum noise levels for a given speed, based on a given traffic mix. 
$5: 00$

J12. Elevated measurement of traffic noise above an ideal reverberant city. S. E. Froseth and R. F. Lambert (Department of Electrical Engineering, University of Minnesota, Minneapolis, MN 55455)

Aerial noise measurement methods may be well suited to the determination of spatially averaged traffic noise exposure levels, and could possibly be used as a means of assessing the long-term effectiveness of motor vehicle noise regulations. In this study two theoretical models are developed for some specific aerial measurement situations. Several characteristics of the models are examined. Limited experimental measurements agree well with theoretically predicted results; elevated measured noise levels are nearly proportional to the density of the traffic (in vehicles per unit area) on the city streets.

\title{
Session K. Architectural Acoustics II: Special Session on Open-Plan Schools
}

\author{
David P. Walsh, Chairperson \\ Wilson, Ihrig and Associates, Inc., Oakland, California 94618
}

\section{Panel Discussion}

$$
2: 00-5: 00
$$

Panel Discussion: A discussion of open-plan schools with emphasis on design concepts, physical requirements, user requirements, learning activities, and effects on users. A brief presentation by each of the panelists will be followed by a general discussion including questions and comments from the floor. The discussion will also include information relating to the City of San Diego's current school construction program which has incorporated various open-plan schools.

Panel Members:

John R. Boice

Educational Facilities Laboratories, Inc. , 3000 Sand Hill Rd., Menlo Park, CA 94025

Robert L. Probst

President, Herman Miller Research Corporation 3970 Varsity Dr., Ann Arbor, MI 48104

Ralph J. Askin

Supervising Architectural Advisor, Bureau of School Facilities Planning, State Education Building, 721 Capitol Mall, Sacramento, CA 95814

Julie Fisher

Member, Board of Education, San Diego Unified School District, 3726 Charles St., San Diego, CA 92106

William Lewis

AIA, Vice-President, Deems/Lewis \& Partners, 2901 Fifth Ave., San Diega, CA 92103

Robert S. Gales

Consultant in Acoustics, 1645 Los Altos Rd., San Diego, CA 92109 


\title{
Session L. Engineering Acoustics I: Transducer Design and Calibration
}

\author{
Gordon E. Martin, Chairperson
}

Naval Undersea Center, San Diego, California 92132

\section{Contributed Papers}

\section{$2: 00$}

Iı1. Sensitivity and directivity of piezoceramic tubes with capped or shiclded ends. W. James Trott (Code 8150, Naval Rescarch Laboralory. Washington, DC 20:375)

The electroacoustic sensitivity of cylindrical, piezoceramic tubes has bocn determined in the low-frequency, omnidirectional range for various conditions of poling together with capped, shiclded, and exposed ends. This range is more than a clecade below the first resonance. NRL's computer program SHIP IPeter It. Rogers, NRL Report 7240 (June 1972)| is used in this report to oxamine the diffraction constants for finite length cylindrical radiators and circular pistons in the end of finite length tubes. The effect of diffraction upon the sensitivity of cylindrical clements with capped and shielded ends are calculated for radial. tangential and longitudinal poling.

$$
2: 15
$$

L2. Three-dimensional finite-element normal-mode calculaLions for flextensional underwater acoustic transducers. Jack N. Boone (U.S. Naval Surface Weapons Center, Dahlgren. VA 22448)

A "curvilincar wodge" finite-element has been developed which is broadly applicable to the family of transducers variously classified as "flextionsional" [G. A. Brigham and L. H. Royster, J. Acoust. Soc. Am. 46, 92(A) (1969)1. The essential aspects of the direct development of the finite element in cylindrical coordinates are presented, with emphasis on advantages over elements employing polynomial mappings. The resolution of the problem of infinite energy density at the coordinate singularity is effected under conditions sufficiently general for the solution of problems arising in the mathematical unodeling of flextensional transducers. Results for the short-circuited normal modes are presented for Class II, Class $\Gamma$, and Class $V$ configurations and compared to experimental and other analytical results for these transducers. Good agreement of the predicted spectra with reported experimental data is found. It is demonstrated that constraints in interface compatibility specifications in multicomponent models may produce large errors and involve generally untenable assumptions.

$$
2: 30
$$

L3. New applications of compliant tube technology. G. Brigham (2543 Ames Avenue, Anaheim, CA 92806)

The use of compliant tubes for pressure releasing in underwater electroacoustic transducers and for mechanical beamforming such as in acoustic lenses has been well known and publicized in the past 23 years. Less well known and more recent applications of tubing is in acoustic barriers/baffling, geodesic lensing/wave guiding, sonar array shading, sonar window trussing, and acoustic energy mode conversion such as in anechoic surfacing and/or wave guiding. While the basic acousties/elastodynamics of tube arrays has been extensively developed to a point of near standardization in recent years, the use of the technology in any specific system still requires considerable numerical analysis. This paper discusses the newer applications and presents several examples of tube systems together with comparisons between analysis and measurements.
$2: 45$

L4. Echo reduction in low-frequency calibration using complex cepstrum. Lynn B. Poche, Jr., and Peter H. Rogers (Naval Research Laboratory, Underwater Sound Reference Division, Orlando, FL 32806)

The complex cepstrum technique was investigated for possible use in removing echoes caused by early reflections when low-frequency, peaked-response transducers are calibrated using transient signals. Syntihetic meissurement data produced by a J9 projector driven with a damped sinusoidal pulse and a pressure release reflection are shown. Real measurement data from a low-frequency line array driven by a single-cycle sinusoid with complex surface and boltom reflections are also shown. In both cases, the echoes were successfully removed using the complex cepstrum technique. Data were digitized directly and stored on digital magnetic tape. Oversampling: was reduced by sifting to achieve the correct sampling rate required by the complex cepstrum method. ISupported in par. by the Naval Electronic Systems Command. I

$3: 00$

L5. Automatic comparison calibration of hydrophones using the ambient noise background. FI. M. Merklinger (Defence Research Establishment Atlantic, P. O. Box 1012, Dartmouth. Nova Scotia, Canada, B2Y 3Z7)

A method is described which precisely and automatically compares the sensitivities of two hydrophones. The method is based on the property that the difference of two signals having cqual average power is uncorrelated with the sum of the two signals. For two hydrophones placed in the same noise environment, a feedback circuit may be divised which adjusts the gain in one of the signal paths so that the required null correlation is achieved and maintainet. The circuit may be analog. rligital, or a hybrid combination of analog and digital. Frecuency selectivity may be achieved with two identical filters. The method is particularly applicable to superdirective arrays since precise relative element weights mav be achieved despite unknown or slowly changing hydrophone sensitivities. Under some circumstances data obtained with poorly weighted superdirective arrays may be improved after the telemetry and data recording operations have been completed.

$$
\text { 3:15 }
$$

L6. On designing an optimum sonar window. G. Brigham (2543 Ames Ave. Anaheim, CA 9280i3)

Acoustic transmission through sonar windows can be a serious problem for electrically scanned receiving systems with narrow beams, low side lobes, and very wide bandwidth' field of view. An optimum sonar window for most systems can be designed by simultaneously considering (a) the three-dimensional model of acoustic transmissitivity for a thick, uniform. panel [R. D. Fay, J. Acoust. Soc. Arı. 25, 220-223 (1953)] and (b) the acoustic transmissitivity of free-flooded, elastic, uniform gratings [G. Brigham et al., J. Acoust. Soc. Am. $59,505(P)(1976)]$. Since an optimized uniform panel would probably not take impact loading a trussed paneling is likely. By choosing materials that will be free of lateral coincidence in the sonar bandwidth/field of view, the transmissitivity can 
be held to within $90 \%$ or better with a phase distortion due only to panel inertia. The trussing can be designed rugged but small relative to an acoustic wavelength, with a cross-sectional deformation compliance equal to the compressibility of sea water to eliminate the Rayleigh scattering in the omnidirectional mode and a woight ratio calculated to eliminate the Rayleigh dipole scattering.

L7. Method of improving the performance of a multimode spherical hydrophone operating near a resonant frequency. H. L. Pond. F. A. Alatalo, and S. H. Ko (Naval Underwater Systems Center. New London Laboratory, New London, CT 06320)

A spherical elastic shell surrounded by fluid will be deformed by an imposed acoustic wave. If the shell is made of a piezo- clectric material, a voltage is induced by the strains. Elements of shell insulated from one another act as hydophones. The res] ionses of such a shell can be used to determine the direction of an incoming wave. A simple cosine pattern for normal dellection dominates from low frequencies through the resonant frequency for the first mode except for frequency bands near resonance for the third, lifth, and seventh modes. The simple detection of direction is based on a cosine paltern for the induced voltage. It is shown that the surface of the shell can be divided into selected areas which are insulated lrom one another and such that the sum of voltages from the elements of area is zero for a selected mode. This method is used to remove the effect of the third and most harmful mode and restore the simple cosine voltage pattern in the frequency band near the resonant frequency for the third nude. I Work sujporled by NAVMAT. I

TUESDAY, 16 NOVEMBER 1976 GOLDEN WEST ROOM, 2:00 P.M.

\title{
Session M. Speech Communication II: High Order Units- Production and Perception
}

\author{
Catherine P. Browman, Chairperson \\ Phonetics Laboratory, Linguistics Department, University of California, \\ Los Angeles, California 90024
}

\section{Contributed Papers}

M1. Distribution of timing events in speech production. G. Weismer and D. R. Ingrisano (Indiana University, Speech and Hearing Center. Bloomington, IN 47401)

The timing of articulatory gestures in speech production was studied in three speakers. Each speaker recorded a list of 400 sentences comprised of a randomized arrangement of 4 sentences by 5 emphatic stress conditions in which each sentence-emphatic stress eombination was repeated 20 times One sentence was designated as the target utterance, and the remaining three sentences were included as dummy utterances. Subjects were trained to place emphatic stress on a given content word in each utterance or to produce sentences with normal lexical stress patterns. Spectrograms of each target utterance-emphatic stress combination were prepared and analyzed according to a time normalization procedure in which the time of occurrence of predetermined events in the articulatory sequence was referred to the normalized utterance duration. The hypothesis tested in this investigation-as predicated by Martin's [Psych. Rev. 79, 487-509 (1972)] theory of timing in speech production - was that the repetition variance associated with the normalized "times-of-articulation" would be conditioned by the location of emphatic stress within utterances. Preliminary results tend to support this prediction. [Supported by NIMH Grant \#R03-MH 28705-01.]

\section{$2: 10$}

M2. Syllable timing as a function of position-in-utterance in infant babbling. D. Kimbrough Oller (University of Miami, Mailman Center for Child Development, Miami, FL 33152) and Bruce Smith (University of Washington, Department of Speech, Seattle, WA 98195)

For several years now it has been known that a number of languages show regular variations of syllable duration as a function of position-in-utterance. The most striking fincling has been that final syllable vowels (in English, for instance) are uj) to $125 \mathrm{msec}$ longer than comparable nonfinal syllable vowels. The present study attempts to determine whether or not the same sorts of patterns of syllable cluration obtain in infant utterances. A corpus of reduplicated CVCV-ulterances of several infants were tape-recorded and analyzed spectrographically. The durations of the infant consonants and vowels were compared with the durations of adult consonants and vowels as pronounced by adult speakers who were asked to read sequences phonetically like the infant utterances. The results show only a slight tendency toward final syllable lengthening in the infant utterances, a tendency which is demonstrably less than that which occurred in the comparable adult utterances. (Work supported by NIH-NICHD contract HD-3-2793 and Grant 5R01 HD 09906-02.]

\section{$2: 20$}

M3. Temporal patterns of syllables, Linda R. Shockey and Ignatius G. Mattingly* (Haskins Laboratories, 270 Crown St. , New Haven, CT 06511)

Comparisons of the temporal patterns of pairs of syllables differing only as to presence versus absence of a particular consonant suggests that the temporal effect of the consonantal articulation is not necessarily either additive or simply related to the duration of the acoustic segment conventionally associated with the consonant. In a study of such temporal effects various monosyllables were embedded in turn in a short carrier sentence, and each version of the sentence was read 30 times at a carefully controlled tempo by one speaker. Durations of segments, syllables and breath-groups were measured from oscillograms, and least pairs, e.g., say versus slay, compared. It appears that final stop or final nasal lengthens both syllable and breath group by an amount roughly equal to the durations of the corresponding acoustic segment; an 
initial stop, liquid or aspirate induces no appreciable lengthening, other segments in the syllable being sharply reduced; and initial /s/ lengthens the syllable breath group by an amount approaching the duration of the fricative segment, the durations of the other acoustic segments being slightly reduced. It is hoped that these results will contribute to more adequate synthesis by rule. (Work supported by $\mathrm{NIH}$ and VA.l *Also Department of Linguistics, University of Connecticut.

M4. Use of nonsense-syllable mimicry in the study of prosodic phenomena. Mark Y. Liberman and Lynn A. Streeter (Bell Laboratories, Murray Hill, NJ 07974)

The technique of nonsense-syllable mimicry of natural utter ances lused by Lindbloom and Rapp, Publ. No. 21, Institute of Linguistics, University of Stockholm (1973) (unpublished)] has many advantages in the study of prosodic phenomena, especially duration. In analytic studies, the elimination of segmental effects as a factor makes data collection much more efficient, and requires only one segmentation criterion. In perceptual studies, the technique eliminates lexical information without unnatural distortions of the signal. In a series of validation experiments, we have found that: (a) the patterns of duration obtained by using this technique were stable and reproducible within and across speakers; (b) mimicry of different natural models with identical stress patterns and constituent structures produced indistinguishable nonsense-syllable duration patterns; (c) obtained duration patterns correspond closely to results of work on natural speech by ourselves and others.

\section{$2: 40$}

M5. Syntactic boundaries in the timing of trochaic speech. William E. Cooper, Steven G. Lapointe, and Jeanne M. Paccia (Research Laboratory of Electronics, Massachusetts Institute of Technology, Cambridge, MA 02139)

The duration of a stressed syllable is shortened when it is immediately followed by an unstressed syllable. Previous work showed that this effect operates across word boundaries but is diminished in magnitude by the presence of an intervening syntactic boundary. In this study, the durations of key segments within stressed syllables were measured in sentence pairs containing a matched phonetic environment. The results for ten speakers showed that the shortening effect was blocked in the presence of a number of major syntactic boundaries, including the boundaries between a noun phrase (NP) and a prepositional phrase (PP), between two PP's, and between a NP or PP and a separate clause. Lesser syntactic boundaries, including the boundaries between a verb and NP direct object and between an NP direct object and NP indirect.object, did not block the shortening rule. The magnitude of the blocking effect did not depend on the transformational history or internal structure of the syntactic constituents as much as on the boundary type.

$$
2: 50
$$

M6. Syntactic determinants of word duration in speech. Richard Goldhor (Research Laboratory of Electronics, 36-575, Massachusetts Institute of Technology, Cambridge, MA 02139)

A preliminary identification of the primary syntactic determinants of duration in English declarative sentences has been made. A hundred declarative sentences were generated from a small set of words, and the durational variations of those words which appeared in more than six sentences were studied. The three syntactic determinants which were found to most strongly and consistently affect word duration are: (1) the length of the phrase in which a word appears; (2) the position of the phrase in its dominating clause; and (3) the number and type of clauses in the sentence. Additionally, these determinants were found to affect phrase-final words differently than phrase-nonfinal words. These findings have been incorporated into a simple prosodic durational algorithm suitable for use in a text-to-speech system.

\section{$3: 00$}

M7. Reading speed as a clue to text structure. M. O. Harris (Acoustics Research Department, Bell Laboratories, Murray Hill, NJ 07974)

Text consists of structured content. Do readers reflect that structure in their production? A system is under design to map the content pattern of a given text, sentence by sentence, thus locating major and lesser topic koundaries. The system depends on word frequency and distribution in that text, and on the recognition of synonyms and other forms of reference, which establish links betwen text segrnents. Acoustic tools help to identify significant variations in reading performance, and these can then be interpreted in terms of the text structure. Material for the present paper consists primarily of four passages, each read by three speakers; about an hours' reading in all. The passages are structurally independent excerpts from much larger works. All of the sentences were randomized together and read as one list, and then each passage was read in original form. A study based on duration measurements, indicates clear agreement among speakers as to the locations where reading speed changes occur: e.g., in sentences of transition between major topics, and at points of salient semantic shift. However, the nature of the changeincrease or decrease in speed is highly speaker dependent.

$$
3: 10
$$

M8. Perceptual phonetics of coarticulation. J. G. Martin, R. H. Meltzer, and C. B. Mills (Depastment of Psychology, University of Maryland, College Park, MD 20742)

In experiments reported earlier at ASA meetings (November, 1975) and elsewhere, reaction time (FiT) of subjects monitoring stop-consonant phoneme targets in tape-recorded sentences was observed. RT was compared when the target (a) was carried by the normal, intact sentence version or (b) was temporally displaced by experimental intervention, that is, separated from prior sentence context by addition of 200 msec to the normal pre-stop-consonant silent interval. Faster RT to temporally displaced than to normal targets was interpreted in terms of coarticulatory cues to target existing in the speech interval preceding the intervention which were used to anticipate the target across the intervention interval. In further analysis, data were separated on the basis of four classes of pretarget phonetic context: stop, fricative, sonorant, and vowel. All classes produced coarticulatory effects (relatively faster RT to displaced compared to normal targets), some more than others. Additional analysis indicated similar effects in the normal sentence versions also. Discussion concerns the mapping of perceptual results onto acoustic and articulatory data. [Work supported by NIMH, ARIBSS.]

$$
3: 20
$$

M9. Factors affecting the detection of mispronunciations. R. A. Cole and J. A. Jakimik (Departrient of Psychology, Carnegie-Mellon University, Pittsburgh, PA 15213)

In a series of experiments, subjects were presented with sentences or short stories, and required to press a response key whenever they detected a mispronounced word. Mispronunciations were produced by changing one phonetic segment in a word to produce a nonword. Detection of mispronunciations was effected by factors at severial linguistic levels: (a) the type of phonetic change, (b) the position of the changed segment in the word, (c) the stress of the syllable (e.g. . "contain" versus "contour", where / $k /$ was pronounced / $g$ / in each word), (d) the syntactic structure of tie sentence, and (e) whether the word containing the mispronunciation occurred in a previous sentence. The results will be summarized and discussed.

$$
3: 30
$$

M10. Word-letter phenomenon with speech stimuli: a wordsegment effect. Joseph Rogers (Department of Psychology, University of California at San Diego, La Jolla, CA 92093) 
and Anne B. Thistle and Brooke Neilson (Department of Linguistics. University of California at San Diego, La Jolla, CA 92093)

Single speech segments excised from natural speech were significantly better identified when embedded in words than when embedded in incomplete words or presented alone. This held true, however, only when a pre- and post-exposure mask designed to contain a randomly ordered selection of the features of speech segments was employed. Under no-mask or white-noise-mask conditions, segments showed a slight advantage over words. These findings parallel those previously reported for visual stimuli: single letters in word context are significantly better identified than single letters alone, but only with random letter-feature masking. Without such masking there is a slight advantage for letters alone. This "wordletter phenomenon" has been offered as evidence for heirachical processing in vision. Likewise, a word-segment effect suggests at least some form of heirarchical processing in speech perception. Further, the parallel results of both visual and auditory studies indicate that auditory and visual language processing channels are either remarkably similar or converge at a relatively early stage. [Work supported by a UCSD Academic Senate Grant to James L. McClelland.]

$$
3: 40
$$

M11. Test of speech intelligibility in noise using sentences with controlled word predictability. D. N. Kalikow and K. N. Stevens (Bolt Beranek and Newman Inc., Cambridge, MA 02138) and L. L. Elliott (Northwestern University, Evanston, IL 60201)

This paper describes a test of everyday speech reception, in which a listener's utilization of the linguistic-situational information of speech is assessed, and is compared with the utilization of acoustic-phonetic information. The test items are sentences which are presented in babble-type noise, and the listener response is the final word in the sentence (the key word) which is always a monosyllabic noun. Two types of sentences are used: high-predictability items for which the key word is somewhat predictable from the context, and lowpredictability items for which the final word camot be predicted from the context. Both types are included in several 50 -item forms of the test, which are balanced for intelligibility, key-word familiarity and predictability, phonetic content, and length. Performance of normally-hearing listeners for various signal-to-noise ratios shows significantly different functions for low- and high-predictability items. The potential application of this test to assessment of speech reception in the hearing impaired is discussed. [Work supported by NTNCDS.]

$$
3: 50
$$

M12. Performance of children from 11 to 17 years of age on a sentence test of speech intelligibility in noise. L. L. Elliott (Audiology Program, Northwestern University, Evanston, IL 60201)

Ninety-six normally hearing children (24 each at ages 11, 13,15 , and 17 years) were individually administered the Speech Perception in Noise (SPIN) Test (see preceding paper by Kalikow, Stevens, and Elliott) at signal-to-noise (STN) levels of 5,0 , and $-5 \mathrm{~dB}$ in counterbalanced order and in quiet. Their task was to repeat the last word of each sentence. Average performance of the 17-year old Ss was very similar to performance of adults on all conditions. An age effect appeared in the results for the high-predictability sentences at $0-d B$ $\mathrm{S} / \mathrm{N}$ level, with younger $\mathrm{Ss}$ showing poorer performance. This age effect was specific to the listening-in-noise condition, since the 11-year-old Ss demonstrated perfect or near perfect scores when later tested in quiet. Implications of these findings will be discussed and the results of testing some children with learning disabilities will also be presented. [Supported by a grant from B.E.H.]
M13. Hearing "words" without words: speech prosody and word perception. Lloyd H. Nakatani and Judith A. Schaffer (Bell Laboratories, Murray Hill, NJ 07974)

The role of prosody in word perception was studied by asking listeners to locate word boundaries in speech with natural prosody but without phonotactic or semantic cues. To obtain such speech, seven speakers read sentences (e.g., "The noisy dog never stopped barking") with "ma" substituted for each syllable of the three-syllable adjective-noun phrase (e.g. :"The mama ma never stopped barking"). These nonsense phrases with all possible stress patterns were excised and played to 38 naive listeners who judged whether they heard each phrase as "ma mama" or "mama ma." The stress pattern determined how well the phrases were parsed into words. When the stress pattern made the word division unambiguous $(01-1,1-12,1-10$ where $0,2,1$ denote no stress, midstress, and high stress), listeners used the pattern to parse the phrases easily; striking individual differences were observed. When the stress pattern left the word division ambiguous $(11-1,1-11,12-1,1-21,10-1,1-01)$, parsing was harder but still better than chance. So not only global patterns, but also detailed information about prosody, are used in word perception.

$4: 10$

M14. Word juncture perception as a function of word-initial phonemes. Kathleen D. Dukes and Lloyd H. Nakatani (Bell Laboratories, Murray Hill, NJ 07974)

A previous study showed that allophonic variations in wordinitial phonemes were the primary cues for word juncture perception. This study examined juncture perception as a function of the word-initial phonemes occurring singly and in clusters. Pairs of two-word phrases contrasting in juncture location (e.g. , "he praises" and "heap raises") and representing a wide variety of word-initial vowels, consonants and consonant clusters were recorded in sentence contexts by four talkers. The phrases were excised from the sentences and played to listeners who indicated which phrase they heard. Juncture perception was good for word-initial voiceless stops, vowels, affricates, and nasals, and fair for fricatives and voiced stops. Juncture perception was poor for liquids and semivowels, but good when they occurred in a consonant cluster. Juncture perception for clusters beginning with /s/ was better than for $/ \mathrm{s} /$ alone. Juncture perception was poor when the same consonant occurred before and after the juncture (e.g. , "heap praises"); this suggests that segmental duration is not an important cue for juncture perception.

$$
4: 20
$$

M15. Perceptual determinants of phrase boundary placement. Lynn A. Streeter (Bell Laboratories, Murray Hill, NJ 07974)

In speech production many acoustic cues are correlated with the presence of a major syntactic boundary. Here the relative contribution of three acoustic cues, fundamental frequency contour, duration, and amplitude to perceptual segmentation process was evaluated. The stimuli were ambiguous algebraic expressions, such as " $A$ times $E$ plus $0, "$ in which the middle term $E$ could be a member of either the first or the second phrase. Two levels (appropriate and neutral) of each of the three cues were varied in a complete factorial design. The results indicated that while all three cues significantly influenced the placement of a phrase boundary, duration was more potent than either amplitude or the fundamental frequency contour.

\section{$4: 30$}

M16. How is the memory search of a two-clause complex sentence in immediate memory carried out? Leonard Shedletsky (Department of Speech, University of Connecticut, Stamford, CT 06903)

An item recognition task was performed with 32 native English speaking, adult, right-handed subjects who listened 
to eight two-clause complex sentences presented to the left ear, each immediately followed by a probe word presented to the right ear. The subjects indicated whether or not the probe word occurred in the sentence and their recognition latency was measured. An analysis of variance was performed on recognition latency as a function of the three independent variables: (a) the serial position of the target word, early or late, within (b) a main or subordinate clause, in (c) initial or final clause position. The findings of this experiment were: (a) a word in the final clause is recognized significantly faster than a word in the initial clause; (b) for subordinate clauses, subjects take longer to respond to a target word occurring late in the clause than to a target word occurring early in the clause; for main clauses, subjects take longer to respond to a target word occurring early in the clause than to a target word occurring late in the clause. Present storage models of sentence processing and memory search models are inadequate to account for all the data. A combined storage-search account was proposed. A serial self-terminating model of clause accessing, with final clause search oecurring prior to initial clause search, fit the data better tharı a simultaneous search of both clauses. Clauses are searched either in a primary or a secondary buffer, depending on clatise type (main or subordinate) and clause position (initial or final) in the sentence. To explain the difference in mode of search between main and subordinate clauses, it was suggested that main clauses exhibit a property of primacy over subordinate clauses.

\title{
Session N. Shock and Vibration II: Vibration and Signature Monitoring II: Analysis
}

\author{
Eric E. Ungar, Chairperson \\ Bolt Beranek and Newman, Inc., Cambridge, Massachusetts 02138
}

\section{Invited Papers}

\author{
$2: 00$
}

N1. Spectrum analysis - a diagnostic tool for high speed rotating machinery. Meherwan $P$. Boyce (Gas Turbine Laboratories Texas A\& M University, College Station, TX 77840)

The role of the spectrum analyzer as a tool in the location of problem areas is findirg greater acceptance in the field of high-speed rotating machinery. Its use as a diagnostic instrument has been limited due to an inadequate understanding of the various signals. To achieve a better understanding of the signed analysis of the various types of transducers and their effect on signature analysis is discussed. The development of data banks to enhance the understanding of various types of signals is described. This data bank is developed by isolating individual problems and recording their signature patterns. These signature patterns can be used both visually or programed for computer matching. In this manner the spectrum analyzer can be used as on line diagnostic system. Test patterns and actual field tests are described to show the impact that such a system would have when combined with performance data as a diagnostic tool.

$$
2: 30
$$

N2. Automated vibration signature analysis. J. L. Frarey (Product Development, Shaker Research Corporation, Ballston Lake, NY 12019)

Diagnosing the condition of a machine by its vibration signature has a great deal of appeal. The problems that have been encountered in its widespread use involve the availability of analysis equipment and the skill of the data analyst. Combining a real time analyzer with a computer can process a gr eat amount of data quickly and reduce the drudgery of analysis. Single-frequency Fourier component calculation may easily be made in the computer and can take the place of tracking filters and phase meters. This ability to process, store, ancl recall data quickly, coupled with the ability to program a computer to recognize basic problem symptoms, is making automated signature analysis for machine diagnostics possible, Hardware and software examples demonstrating this capability are presented to support the conclusions.

$$
3: 00
$$

N3. Use of time waveform characteristics and phase measurements for vibration signa.l analysis. J. B. Catlin (IRD Mechanalysis, Inc., 6150 Huntley Rd., Columbus, OH 432 49 )

Comparisons are made betwen amplitude versus frequency and amplitude versus time analyses for typical machinery generated vibrations such as unbalance, misalignment, oil whirl, and aerodynamic/hydraulic pulsations. The information provided in each case shows the extent to which the amplitude versus time supplements the amplitude versus frequency 
analysis. Transient and non-steady-state vibrations are shown to be particularly well suited to amplitude versus time analysis; providing considerably more information than amplitude versus frequency. In particular, examples include machine criticals, beat frequencies, and electrical unbalance. Various combinations of basic vibration waveforms are used to suggest standardized techniques which can aid in simplifying interpretation of actual vibration data. The use of phase measurements, as obtained from time wave forms and as a direct measurement, is described for balancing and detection of machine defects.

N4. Feature enhancement of vibration signatures utilizing synchronous spectrum averaging. D. J. Bozich and A.C. Keller (Spectral Dynamics Corp. of San Diego, San Diego, CA 92112)

Vibration response signals containing periodic components such as obtained from rotating equipment, gears, etc., can be effectively analyzed in the frequency domain. The averaging of spectra wherein the Fourier transform operation is synchronized with a particular periodic component, will reinforce that frequency component and all of its harmonics, while all nonsynchronous data will tend to be nonreinforced, thereby enhancing the synchronous spectral components. Features such as gear teeth and turbine blade passing frequencies, etc., and their related sidebands, can be extracted as signatures for baseline comparison and fault evaluation. This paper will discuss signatures obtained from averaged spectra and the related set of synchronous spectra; i. e., a synchronous average spectrum for each periodic component of interest.

N5. Parameters to be considered in machinery vibration monitoring systems. R. M. Gorman and K. A. Bowen (Electric Boat Division of General Dynamics, Groton, CT 06340)

Vibration monitoring systems are being used in many applications to aid in predicting incipient machinery failure. This presentation addresses the significant choices which have to be made in the selection of such a system. The number of vibration transducers to be. monitored and their locations on particular machines are discussed. A comparison is made of various frequency ranges and filter bandwidths which can be used in analysis of the monitored signals. Other areas discussed are the determination of the time interval between measurements, the allowable increases in vibration levels before repair is required, and the applications of trend analysis in monitoring systems.

\title{
Session O. Physical Acoustics III: Atmospheric Acoustics II
}

\author{
T. F. W. Embleton, Chairperson
}

National Research Council, Montreal Road, Ottawa, Ontario K1A 051, Canada

\section{Invited Papers}

\author{
$8: 30$
}

01. Interpretation of sodar measurements: relevant properties of the atmosphere's planetary boundary layer. D. W. Thomson (Department of Meteorology, The Pennsylvania State University, University Park, PA 16802)

Assuming a Kolmogorov spectrum of turbulence, the acoustic scattering cross section may be written as a function of incident wavelength, scattering angle, and the magnitude of the root-mean-square turbulent temperature and velocity fluctuations. In the "surface" layer SL ( $\max \approx 3-30 \mathrm{~m}$ height) of the atmosphere the statistical properties of turbulent velocity, temperature, humidity, and pressure fluctuations above many surface types are well understood for a wide variety of meteorological conditions. Hence, it is possible using sodar to derive unique, quantitative measurements of SL atmospheric turbulence. Detailed information about planetary boundary layer PBL $(\max \simeq 300 \mathrm{~m}-2 \mathrm{~km}$ height) turbulence is not required in order to make continuous sodar measurements of vertical profiles of turbulencerelated atmospheric structure and winds (from signal Doppler shifts). Sodar is, thus, now routinely used, e.g. , for air pollution related, micrometeorological research, and wind-shear detection measurements. Our perception of many complicated PBL structural features and processes has been greatly expanded by the now familiar vertical-time section sodar records of various "layers" and thermal convection. However, except for "unstable" 
conditions of free convection, SL turbulence models are not generally applicable for the quantitative interpretation of PBL sodar data. In order to realize the full indirect sounding potential of sodar during "stable" atmospheric conditions, it is necessary to determine the relationship(s) between observed "macroscale" features such as multiple layers (with ind without waves), intermittant "patches" of turbulence and "bursts" of vertical momentun or heat transport and the space-time dependent properties of the turbulence in such situations. This paper first reviews the application of contemporary SL and PBL models to the interpretation of sodar measurements. Functional relationships between sodar observed and m.xdel Implicit parameters are summarized. Finally, the application of sodar for development and verification of selected atmospheric models is discussed. [Research supported by EPA Grant R800:397.1

\section{Contributed Papers}

$$
\text { 9:00 }
$$

O2. Measurement of the rate of dissipation of turbulent kinetic energy in convective conditions by acoustic sounding. S.J. Caughey and B. A. Crease (Meteorological Research Unit, RAF, Cardington, Bedford, MA), D. N. Asimakopoulos and R.S. Cole (Department of Electronic and Electrical Engineering, University College, London, ENG)

In the bistatic configuration acoustic sounding offers a remote method for determining $C_{V}^{2}$, the structure parameter of the wind field and hence (assuming a value for the inertial subrange constant $\alpha$ of 0.5 ) for estimating the rate of dissipation of turbulent kinetic energy $\epsilon$, since (see, e.g., Kaimal Boundary Layer Meteorol. 4, 298-309 (1973)] $C_{V}^{2}=4 \alpha \epsilon^{2 / 3}$ $\approx 2 \epsilon^{2 / 3}$. Direct estimates of $\epsilon$ and $C_{T}^{2}$ (the structure parameter for temperature) were made using turbulence probes Isee Readings and Butler, Meteorol. Mag. 100, 286-298 (1972)] attached to the tethering cable of a large $\left(1300 \mathrm{~m}^{3}\right)$ balloon. Sounder derived values were obtained using two carefully calibrated acoustic arrays. The second array was oriented at $45^{\circ}$ to the vertical and positioned to record the off-axis $\left(135^{\circ}\right)$ scattered sound from the transmission of the first at $100 \mathrm{~m}$ height. In applying the bistatic radar equation the term involving $C_{T}^{2}$ was found negligible and excellent agreement was obtained between the sounder and directly measured $\epsilon$ values.

\section{9:15}

03. Atmospheric turbulence as observed by in situ measurements and Doppler Sodar, A. Weill and F. Baudin (CNET, CRPE, 38-40 rue du Général Leclerc, 92131-Issy-lesMoulineaux, France)

The rate of dissipation by turbulent fluctuations and eddy flux of momentum are measured in the atmospheric boundary layer with the Doppler Sodar of the CNET and compared with hot-wire anemometer measurements. Different hypothesis and methods to calculate turbulence parameters will be first discussed with special emphasis on the possibilities offered by a triple Doppler Sodar. Then typical observations of eddy parameters and of their evolution will be presented. [Work supported by CNET/CR PE/CNRS. I

$$
9: 30
$$

04. Pibal/acoustic radar examination of Arctic boundarylayer winds. F.D. Carsey LArctic Ice Dynamics Joint Experiment (ADDJEX), University of Washington, 4059 Roosevelt Way N. E. , Seattle, WA 98195]

Pibal balloon wind data taken over Arctic pack ice is normalized using the acoustic radar derived boundary layer thickness. The resulting winds can be averaged to yield a well behaved profile reflective of seasonal stability and heat flux. Examination of the original and the constructed mean profiles gives information on the character of the transport and the nature of the eddies in the planetary boundary layer. In particular, regimes of constant stress and of secondary flow are identifiahle. A technique for using acoustic radar derived heights and synoptic meteorological data to obtain air stress profiles is developed. [Work supported by NSF and NOAA.]
$9: 45$

O5. Experimental study of an echosonde interferometer for remote sensing of atmospheric temperatures. Edmund $\mathrm{H}$. Brown (Wave Propagation Laboratory. NOAA, Boulder, CO 80302) and Wayne M. Wright* (Cooperative Institute for Research in Environmental Sciences, Boulder, CO 80302)

We have constructed a preliminary version of the oombined echosonde interferometer suggested by Brown and Little [E.H. Brown and C.G. Little, Nature (to appear)]. Here, we give a description of this device (which for brevity we call an "echoferometer") and present some of: the first results obtained with it. The fact that the formation of an acoustic interference pattern from a pair of coherent sources can be combined with the scattering of sound to a receiver ky turbulent refractivity fluctuations (as in an echosonde) is of interest in itself. Moreover, application of the effect to remcte sensing of atmospheric temperature profiles would add an axtremely valuable tool to meteorological research if sufficient sensitivity could be achieved. We include some possible schemes, such as correlation detection, for progress towards, such a goal, together with a sketch of an engineering feasibility study.

*Permanent address: Kalamazoo College, Kalamazoo, MI 49007.

\section{0:00}

O6. Vibrational energy transition rates from recent sound absorption measurements in moist air and in moist nitrogen. H. E. Bass and F.D. Shields (Department of Physics and Astronomy, University of Mississippi, University, MS 38677)

Recent measurements of sound abscrption in moist air at temperatures between $0^{\circ} \mathrm{F}$ and $100^{\circ} \mathrm{F}$ have been analyzed to extract that part of the absorption due to the vibrational relaxation of oxygen. The measurements extend to higher water-vapor concentrations than most previous measurements and show the shift in frequency of maximum relaxation absorption per wavelength with percent water vapor to be less than that extrapolated from previous rneasurements. The $f_{\max }$ versus percent- $\mathrm{H}_{2} \mathrm{O}$ curve has been analyzed in conjunction with previous measurements in binary mixtures of $\mathrm{H}_{2} \mathrm{O}$ and $\mathrm{O}_{2}$ to give more accurate values for the rate of $\mathrm{v}-\mathrm{v}$ exchange between $\mathrm{H}_{2} \mathrm{O}$ and $\mathrm{O}_{2}$ and for the $\mathrm{v}-\mathrm{R}$, $t$ rate in $\mathrm{H}_{2} \mathrm{O}-$ $\mathrm{H}_{2} \mathrm{O}$ collisions. Measurements have also been made of the vibrational relaxation absorption in moist nitrogen at 100 , 200 , and $300^{\circ} \mathrm{F}$. In these measuremerts $f_{\max }$ was found to increase linearly with $\mathrm{H}_{2} \mathrm{O}$ concentration and a more accurate value for the $v-R, t$ (or $v$ ) rate for $\mathrm{H}_{2}$ ) deexciting $\mathrm{N}_{2}$ vibration has been determined at each temLerature. [Work supported NASA-Langley Research Center and NASA-Lewis Research Center.]

07. Laboratory measurements of atmospheric absorption in air. M. C. Lee and L.C. Sutherland (Wyle Research, El Segundo, CA 90245)

Laboratory measurements of atmospheric absorption in moist air are reported at frequencies from about 4 to $400 \mathrm{~Hz} \gamma^{\prime}$ atm. These measurements provided data for frequencies below the range of any previously publ ished results. They 
were observed in a resonance tube apparatus at pressures up to $100 \mathrm{~atm}$. Initial measurements of boundary losses in the tube, measured with argon, agreed closely with predicted values based on current theory. This accounted for finite acoustic propagation through the tube wall and "real gas" effects, as well as viscous and thermal losses. The measured values of absorption in air and the corresponding molecular relaxation frequencies provided new validation at very low frequencies for a standard prediction model on air absorption in preparation by the S1-57 Committee on Sound Propagation. [The research was supported in part by the U.S. Army Reresearch Office, Research Triangle Park, NC.]

\section{0:30-BREAK}

$10: 45$

08. Application of acoustic sounding to estimating fusion in an atmospheric boundary layer. Bryan R. Kerman (Boundary Layer Research Division, Atmospheric Environment Service, 4905 Dufferin St. , Downsview, Canada M3H 5T4)

An acoustic sounder system can be configured to provide estimates of the vertical profile of velocity and dissipation rate of kinetic and potential energy. A method based on the concepts of boundary-layer similarity theory is outlined by which the characteristic velocity in a freely convective boundary layer is deduced from the inversion height and the asymptotic value of the dissipation rate of kinetic energy near the inversion. These values, in conjunction with the mean velocity, are used to establish the normalized heights, downwind and cross-wind distances, and cross-wind integrated concentrations. The normalized lateral and vertical standard deviations are then deduced from empirically established laboratory relationships for nonbuoyant particulates. Preliminary analysis indicate a similar methodology may be applicable to the stable boundary layer.

\section{1:00}

O9. Sodar-derived $C_{T}^{2}$ and $C_{V}^{2}$ compared with simultaneous, in situ aircraft measurements: nighttime and daytime case studies. D.W. Thomson, R. L. Coulter, and Z. Warhaft (Department of Meteorology, The Pennsylvania State University, University Park, PA 16802)

Simultaneous measurements of $C_{T}^{2}$ and $C_{V}^{2}$ have been made using the Penn State Doppler sodar system and comprehensively instrumented research aircraft. In each experiment the aircraft was vectored using air-ground ratio to the altitude (ranging from $\simeq 150$ to $600 \mathrm{~m}$ ) zone of interest on the basis of the received sodar signals. Measurements have been made in both "layers" (with and without detectable turbulence and "waves"), and in convective "plumes". $C_{T}^{2}$ and $C_{V}^{2}$ have been derived both from the calibrated monostatic-bistatic Doppler sodar and airborne sensors. In addition to the normal complement of sensors for state parameters, winds, and position, the aircraft was equipped with special, spaced (at sodar $\lambda / 2$ ) hot-wire probes capable of measuring turbulent temperature and velocity fluctuations to about $2.5-\mathrm{cm}$ resolution. Using the combined sodar, turbulence, and meteorological measurements both the turbulent and larger-scale characteristics of the atmosphere in the sounder volume were well defined. Discussion of the results includes suggestions for further experiments involving remote sodar and in situ aircraft measurements. [Research supported by EPA Grant R800397 and ERDA Grant E(11-1)-2463. ]

\section{$11: 15$}

O10. Applications of digital color display techniques for sodar. D. W. Thomson and J. P. Sheib (Department of Meteorology, The Pennsylvania State University, University Park, PA 16802)

The monostatic-bistatic Doppler Sodar at Penn State University has been equipped with a digital color display unit for presenting signal amplitude and Doppler information. Ef- fective system performance for measurements of planetary boundary layer structure and dynamics has been markedly improved. The color technique greatly enhances effective grey-scale resolution in vertical-time section displays of received signal amplitude. Color presentation of signal Doppler information facilitates analysis of wave phenomena and thermal convection in the planetary boundary layer. The system is in use for a variety of field measurements. In some, a research aircraft is radio directed into the region of meteorological interest. Other experiments involve the development of user-oriented color display techniques for ready interpretation of air pollution related parameters. [Research supported by EPA Grant R800397. I

\section{$11: 30$}

011. Processing of airborne acoustic noise sources using passive range determination techniques. G. W. Brooks and N. P. Huffnagle (Martin Marietta Aerospace, P.O. Box 5837, MP-539, Orlando, FI 32805)

This paper presents a continuation of the passive range measurement efforts reported at the 90th conference. Previous work included the modeling of passive range determination using a single point receiver based on moving source observables. The outcome as reported last year was a parameterization of a hypothetical system that had the capability to determine range to source passively. This year, we have expanded the work to include building a fully operational system and field testing it against various moving sources. In addition, a pseudo-Fourier analysis section has been incorporated to accurately and correctly sort out and classify moving sources. This, coupled with $a$ priori selection information coded into the system, enables it to perform those required functions against preselected sources while ignoring nondesired signals. We have concentrated this year on enlarging our data base of acoustic signatures with an eye to determining the points of commonality between major source classes. This has led to vastly simplified signal processing techniques compared with previously tried methods. Specifically, we have found that within a source class, certain fundamental signature components cannot be masked by purely physical means without materially degrading the operation of the source. Masking efforts employing jamming techniques are not as effective as desired, since jamming means that the total acoustic output has been raised yielding a more visible source. Baffling of noise sources, while effective for highfrequency noise components, tends to be less than effective when used to suppress the lower components without an attendant loss in source mobility performance.

\section{1:45}

012. Calculations of the slant visual range (SVR) based on acoustic radar records. Ch. Werner (Institute of Atmospheric Physics (DFVLR), Oberpfaffenhofen, Federal Republic of Germany, D-8031)

A careful measurement of slant visibility becomes every day more important for safe and regular aircraft landing operations. The use of an optical, single-ended radar (lidar) is restricted by safety rules and increasing costs. Monostatic acoustic radars are used by air pollution engineers for control and forecasting. Under the assumption that the interested visibility conditions are given by the temperature and humidity structure of the lowest few hundred meters of the troposphere, the acoustic radar in combination with groundbased visibility meters can be used as a practicable compromise to detect SVR. The monostatic acoustic radar gives information about the inversion layer and the top of low clouds. Calculations based on the measured visibility near the ground and the measured vertical-temperature structure by sodar in addition with the known behavior of the aerosol particles with the relative humidity show that in most cases it would be possible to detect SVR. Some examples of a field experiment are given. 


\title{
Session P. Underwater Acoustics III: Propagation and Fluctuations. Precis Poster Session
}

\author{
Halcyon Morris, Chairperson
}

Naval Undersea Center, San Diego, California 92132

\section{Poster Session}

$8: 30$

P1. Theory of pulse spreading in dispersive random sound channels. F.D. Tappert and L.B. Dozier (New York University, Courant Institute, 251 Mercer St., New York, NY 10012)

The information-carrying capacity of underwater sound channels using a normal-mode, pulse-coded modulation scheme has been theoretically calculated. The effects of random internal wave fluctuations on signal propagation are taken into account. A pulse compression scheme is proposed to intensify received signals. The limiting factor is found to be random coupling between the acoustic normal modes due to seattering from internal wave modes. (Work supported by ONR. 1

$$
8: 34
$$

P2. Coupling coefficients between acoustic wave normal modes and internal wave normal modes. L. B. Dozier and F.D. Tappert (New York University, Courant Institute, 251 Mercer St., New York, NY 10012)

Theories of acoustic signal propagation in randomly fluctuating oceans using normal modes as a basis require knowledge of the coupling coefficients, or matrix elements, that connect the acoustic wave normal modes and internal wave normal modes. We have computed these coupling coefficients numerically using programs that generate both sets of normal modes from model sound velocity profiles and Brunt-Vaisala frequency profiles. Statistical properties of these random coupling coefficients have also been obtained. [Work supported by ONR. $]$

$$
8: 38
$$

P3. Underwater sound wave propagation in the presence of a randomly perturbed profile. I. M. Besier is and W. E. Kohler (Virginia Polytechnic Institute and State University, Blacksburg, VA 24061)

The propagation of acoustic signals over long ranges in the deep ocean is influenced significantly by the following factors: (1) a deterministic sound speed profile possessing a "sound channel"; (b) highly anisotropic spatial random fluctuations; (3) temporal fluctuations due primarily to internal waves. The wave equation, with a randomly perturbed soundspeed profile, is initially considered, and a scaling is introduced which is typical of the ocean environment. A spacetime parabolic equation is then obtained for the random product of complex pressures at two spatial and temporal points. Finally, a transport equation is derived for a suitably chosen transform of the pressure correlation function. This transport equation is general in the sense that it can model the decorrelation of acoustic signals due to offsets in range, azimuth, depth, and time. [Work supported by ONR.]

$$
8: 42
$$

P4. Coherent ray propagation through a Gulf Stream Ring. N. L. Weinberg and X. Zabalgogeazcoa (Institute for Acoustical Research, Miami Div., Palisades Geophysical Institute, 615 S. W. 2nd Ave., Miami, FL 33130)

Coherent ray-tracing techniques were applied to describe acoustic propagation through a Gulf Stream Ring $320 \mathrm{~km}$ in diameter as measured in 1973. Rays were traced from a fixed source to a fixed receiver $445 \mathrm{~km}$ distant incorporating the profile variations of the Ring which had a forward movement of $4 \mathrm{~km}$ a day. This anomaly shifted the deep sound channel axis about $400 \mathrm{~m}$ and also decreased the minimum sound velocity. These profile variations caused significant changes in the arrival pattern of the ray types and number of cycles. A time history is presented of the relationships between the received signal variations and the passage of the Ring including the effects of the changes in multipath structure.

\section{$8: 46$}

P5. Stochastic Green's function approach to acoustic propagation through a fluctuating medium. David P. Vasholz (Code 792 , Naval Coastal Systems Laboratcry, Panama City, FL 32401)

A general, Green's function-based approach to the problem of acoustic wave propagation through a random medium is presented. Starting with the idea that stationarity should be preserved in the propagation from source to field point, the hypothes is that the moments of the stuchastic Green's function $G$ are invariant under arbitrary uniform translation of all time variables is proposed. Proceeding by analogy with quantum field theory the quantities $\mathcal{G}$ and $M$, related to the first and second moments of $G$, are defined. Various properties of $\mathcal{G}$ and $M$ are discussed, and it is shown that they may be conveniently used to describe the principal physical effects induced by a fluctuating medium. An exactly soluble model which illustrates the general results is presented.

$$
8: 50
$$

P6. Applications of a full diffraction theory numerical simulation of sound propagation in the ocean. G. Fain and L.E. Estes (Department of Electrical Engineering, Southeastern Massachusetts University, North Dartmouth, MA 02747)

A review of this numerical technique and its physical basis is presented. A comparison of the technique and the parabolic approximation is made with particular attention paid to broad beam errors in the two approaches. The propagation is simulated in stepwise fashion with the range step sizes being limited by the Rayleigh length. The Rayleigh length is defined for a variety of different sound velocity profiles. The sensitivity of the technique to step size ancl sampling rates is examined by varying these parameters. The theory is extended to include smooth boundaries, sloping: boundaries, and Snell's law bending in range (as well as depth). SOFAR channel, surface, duct, split beam shadow zone and sloping bottom are among the examples presented. [Work partially supported by the Office of Naval Research.]

$$
8: 54
$$

P7. Exact relation between the solutions of the Helmholtz and parabolic equations. J. A. DeSanto (Naval Research Laboratory, Washington, DC 20375)

Given that the velocity potential $\Psi$ satisfies the Helmholtz equation describing sound propagatior. in a waveguide with a depth and range dependent sound speed. Given that the function $p$ satisfies a parabolic partial differential equation (which can be "derived" from the $\Psi$ equation). Wat is the relation between $\Psi$ and $p$ ? It is shown that $\Psi$ can be expressed as an integral transform in range of $p$. For sound speeds which are 
only a function of depth, the integral transform is exact. This is explicitly shown using the normal mode expansion. For sound speeds which depend on both depth and range, the transform involves the solution of an auxiliary partial differential equation. In either case, a stationary phase approximation of the integral transform yields the usual parabolic approximation. Corrections to this stationary phase approximation will be discussed.

$$
8: 58
$$

P8. Effect of Doppler on long-range sound propagation. Jerome A. Neubert (Naval Undersea Center, San Diego, CA 92132)

In this sound propagation study of the effect of Doppler on long-range acoustic tracking, a method of modeling the effects of source and/or receiver motion has been developed. It is based on modilying the inputs to existing normal mode software so that the source and/or receiver trajectory and consequent Doppler effects of the given ocean experiment are properly modeled without requiring direct changes in the static software. From this the effects of the received signal after long range, multipath sound propagation from a moving source can be modeled and interpreted.

9:02

P9. Initial field for the parabolic equation method. D. R, Palmer (Code 8176 , Naval Research Laboratory, Washington, DC 20375)

One of the practical problems which anyone interested in using the parabolic equation method must face is the proper choice for an initial field. In this paper we propose the use of an initial field which is more physical than a Gaussian, far easier to construct numerically than a coherent sum of normal modes, and for which a detailed error analysis is possible.

9:06

P10. Future time prediction of transient signals from limited time measurements using the Prony method. L.G. Beatty and A. Z. Robinson (Naval Research Laboratory, Underwater Sotnd Reference Division, P.O. Box 8337, Orlando, FL 32806)

Reflection interference associated with the reception of acoustic signals imposes low frequency limitations on the information which can be derived from the spectrum of the direct signal if the usual steady-state or Fourier-type processing is employed. To eliminate this interference it would be desirable to be able to predict the future of the direct signal from the measurement of a small number of time samples taken before the arrival of the unwanted echo. One approach to this problem is to seek a closed form solution of the transient wave form in terms of the poles and zeros of its Laplace transform from the time sample measurements. This procedure which is tantamount to expanding the waveform in a series of complex exponentials is called the Prony method. 'Theoretically this expansion should be possible for signals whose transforms have a finite number of poles and żeros. Results of a computer simulation of this process and its implication for underwater sound calibration are discussed. [Work supported in part by the Naval Electronic Systems Command. ]

\section{9:10}

P11. Analysis of shallow water sound propagation by normal mode theory. D. White and D. F. Gordon (Naval Undersea Center, Code 3041, San Diego, CA 92132)

Normal-mode computations agree well with experimental sound propagation data observed in shallow water in the South China Sea. The sound-speed profile has negative gradients in the water over a structured silt bottom. Determining a suffi- cient set of modes can be aided by the relationships between certain fundamental parameters of ray and mode theory. Interference distance between adjacent modes and ray theory loop length can be equated through their phase velocity. The ray theory accuracy can be improved by a frequency correction IE. L. Murphy and J. A. Davis, J. Acoust. Soc. Am. 56, $1747-1760$ (1974) 1. Mode attenuation loss over one loop Iength can be attributed to the loss at one bottom reflection. A bottom reflected phase shift can also be derived from mode theory. Optimum propagation occurs at the lowest frequency that permits a mode to attain maximum loop length.

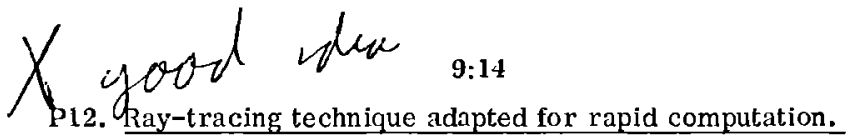
D.w. Princehouse and C. E. Lacy (Applied Physics Laboratory, 1013 N. E. 40th St., Seattle, WA 98105)

REVGEN provides a computerized ocean acoustic environment for the development of a modern active sonar system. [D, W. Princehouse, "Real-time reverberation generator," J. Acoust. Soc. Am. 57, S68(A) (1975). l During an exercise, it is important that it run in as near to real time as possible. We have developed a method whereby the numerically difficult and time-consuming portion of ray tracing can be performed before the exercise starts, and the results used rapidly when needed. We work in the limit of geometrical acoustics and assume that the sound velocity profile depends only on depth, not range or time. While many ray-tracing techniques can be adapted, we have used those of NISSM $\amalg$. [Henry Weinberg. Naval Underwater Systems Center, New London, CT 06320. NUSC Technical Report 4527. I A function of two variables is defined, range versus launch depth and vertex velocity. Knowledge of this function allows ray tracing to be performed. This function may be computed prior to its need, a lengthy process. By encoding it in an efficient fashion, however, it may be stored in computer memory for rapid access when needed. Examples of ray tracing, caustic detection, and multipath propagation will be shown.

\section{9:18}

P13. Separation of individual multipath arrivals - an experiment. T.E. Ewart and J.E. Ehrenberg (Applied Physics Laboratory, University of Washington, $1013 \mathrm{NE} 40$ th St. , Seattle, WA 98195)

During June 1976 an experiment was conducted in which acoustic pulses were transmitted from a fixed transmitter to a fixed receiver 1100 meters distant. A 2-msec pulsed tone and 4-msec Taylor weighted FM slide signals were sent at $2,4,8$, and $16 \mathrm{kHz}$ and the received signals were sampled every $15 \mathrm{msec}$. The sound velocity profile was such that two wholly refracted paths with a travel-time differ ence of about $0.5 \mathrm{msec}$ were received. Analysis of the data shows distinctly different behavior in the phases and amplitudes of the two arrivals. The vertical arrival angle of each ray has been computed from the data received at two vertically spaced transducers and the fluctuations in the angle are discussed. The signal-processing techniques used to obtain the phase and amplitude estimates from the data are presented. The implication of this experiment toward understanding the relationship of multipath signals to the oceanographic environment (e.g., internal waves and fine structure) is discussed.

\section{$9: 22$}

P14. Acoustic phase fluctuations induced by internal waves in the ocean. Yves J.F. Desaubies (Applied Physies Laboratory, University of Washington, 1013 N.E. 40th St., Seattle, WA 98195)

A coustic phase fluctuations induced by internal waves in the ocean are calculated in the geometric approximation. The results compare favorably with available experimental data. 
$9: 26$

P15. Amplitude fluctuations of signals received from $\mathrm{cw}$ sources towed at long ranges in the deep ocean. D.J. Ramsdale, K. D. Flowers, G. V. Frisk, and G.R. Giellis (Naval Research Laboratory, Washington, DC 20375)

In April 1974, three $\mathrm{cw}$ sources (9.807, 110, and $262 \mathrm{~Hz}$ ) were towed for 16 days in the Atlantic along a track from Antigua to the Grand Banks of Newfoundland. Data from these sources were recorded near Bermuda, Puerto Rico, and Antigua on both bottom and suspended hydrophones. A 3-h running average was subtracted from the amplitude data in order to remove long-term deterministic trends. The remaining fluctuations were analyzed in fixed time segments to determine the correlation time, the density function and the associated first four moments. The variate difference technique was used to estimate the degree of randomness of the fluctuations. Finally, the effects of range, range-rate, source frequency, and environment on the fluctuation statisties are discussed.

$$
\text { 9:30 }
$$

P16. Probability density functions for the amplitude of signals received from $\mathrm{cw}$ sources tow'ed at long ranges in the deep ocean. G. V. Frisk, G. R. Giellis, D.J. Ramsdale, and K. D. Flowers (Naval Research Laboratory, Washington, DC 20375)

The characteristies of the probability density functions for the $\mathrm{cw}$ tow data of the previous paper are discussed. Theoretical density functions which best fit the data and their physical interpretation are presented. The properties of the corresponding log transformed density functions are also discussed.

P17. Low-frequency shallow water propagation in Barrow Strait, N.W.T., Canada. R. F. MacKinmon and J. M. Ozard (Defence Research Establishment Pacific, F.M.O., Victoria, BC, Canada)

A study is made of wave dispersion from explosive charges over ranges of $7-15 \mathrm{~km}$ in water of $150-\mathrm{m}$ depth at a location in the Canadian Arctic archipelago. At frequencies below 40 $\mathrm{Hz}$, speeds are found to be as high as $7.4 \mathrm{~km} / \mathrm{sec}$ and lower than $0.8 \mathrm{~km} / \mathrm{sec}$ with a particularly large arrival occurring at a speed of $1.2 \mathrm{~km} / \mathrm{sec}$. At higher frequencies most energy travels at speeds near the speed of sound in water. Independent seismic surveys have shown the area of interest to be characterized by nearly horizontal strata in the bottom consisting of an extremely thin cover of unconsolidated sediment over layers of Paleozoic carbonates. General features of the observed wave trains are discussed on the basis of a theoretical three-layer model with a bottom consisting of two elastic layers. Attenuation is observed to be severe generally and as a function of frequency possesses a maximum near $30 \mathrm{~Hz}$.

$$
\text { 9:38 }
$$

P18. Sound transmission through the sea surface. J.E. Barger, D. A. Sachs, and J.R. Nitsche (Bolt Beranek and Newman Inc., 50 Moulton St., Cambridge, MA 02138)

Under conditions of sea state $2.5-3$ with significant swell, cw-tone bursts from a helicopter-borne calibrated acoustic horn were received by a vertical string of hydrophones, and the effect of the rough air-water interface on the acoustic transmission was measured. The experiments covered a range of parameters comparable to those previously reported at the 90th Meeting of the Acoustical Society for experiments with scaled sea surfaces [J. Acoust. Soc. Am. 58, S86(A) (1975)]; namely : acoustic source frequencies of 220,450 , and $1000 \mathrm{~Hz}$, grazing angles from $\sim 3^{\circ}-5^{\circ}$ to $\sim 20^{\circ}$, and source heights from 50 to $4000 \mathrm{ft}$. The sound path was oriented parallel and antiparallel to the sea direction. Transmission losses relative to smooth surface transmission from 2 to $8 \mathrm{~dB}$ were observed at 220 and $450 \mathrm{~Hz}$ at heights up to $1000 \mathrm{ft}$. At $1000 \mathrm{~Hz}$, transmission was generally close to or better than flat surface for source altitudes below $4000 \mathrm{ft}$. The transmission at all frequencies generally improved at the highest altitude. The data were found to compare well with the results of the scaled experiments under similar conditions. [Work supported by DARPA and monitored by ONR.

$$
\text { 9:42 }
$$

P19. Sound attenuation by conglomerites of bubbles at low frequencies. K.J. Diercks and W. B. Huckabay (Applied Research Laboratories, The University of Texas at Austin, P. O. Box 8029, Austin, TX 78712)

Experimental measurements of soind attenuation in the frequency range from 0.5 to $10.0 \mathrm{kHz}$ are described. Small plastic bubbles simulating in volume that of an adult anchovy swimbladder were used. Both signal enhancements and attenuations ranging from +5 to $-25 \mathrm{~dB}$, respectively, we re observed. Effects of bubble location and population density upon the frequency and magnitude of the maximum enhancement or attenuation are described and a simple mathematical model relating these parameters is presented. [Work supported by NAVSEA.]

\section{$9: 46$}

P20. Volume attenuation in the Labrador Basin. I. A. Fraser and J.B. Franklin (Defence Research Establishment Atlantic, P.O. Box 1012, Dartmouth, Nova Sectia, Canada B2Y 3Z7)

Volume attenuation coefficients for one-third octave frequency bands up to $400 \mathrm{~Hz}$ have been determined as part of an underwater sound propagation study conducted in the Labrador Basin in cooperation with the U.S. Naval Research Laboratory. Charge detonation depths, recelver depth and the sound channel axis depth were all roughly $2100 \mathrm{~m}$ along the $1300-\mathrm{km}$ track. A late summer maximum in the near-surface sound velocity and a water depth greater then $3000 \mathrm{~m}$ also helped to ensure nearly ideal measurement conditions. The deduced volume attenuation coefficients were greater than the Thorp predictions [W.H. Thorp, J. Acoust. Soc. Am. 42, 270 (1967)], with attenuation increasing toward both higher and lower frequencies from a minimum $(0.002 \mathrm{~dB} / \mathrm{km})$ near $60 \mathrm{~Hz}$. Comparisons with results from other areas will be made. Results on the low frequency dependence of propagation loss will also be discussed.

$$
\text { 9:50 }
$$

P21. Low-frequency attenuation in the North Atlantic Ocean. A. C. Kibblewhite (Department of Phy sics, The University of Auckland, Auckland, New Zealand) and N. R. Bedford and S. K. Mitchell (Applied Research Laboratories, The University of Texas at Austin, Austin, TX 78712)

Several Pacific Ocean experiments have been reported in which the attenuation of underwater sciund at low frequencies is apparently regionally dependent. An experiment conducted in the North Atlantic Ocean in 1973 has provided data for use in determining attenuation coefficients for frequencies below 300 $\mathrm{Hz}$ for that region. The methods used to obtain these coefficients, their values, and their relationship to previously reported data from other regions will be presented.

$$
\text { 9:54 }
$$

P22. Excess attenuation in a stochastic environment: theory, model, and experiment, H. G. Schneider (Forschungsanstalt der Bundeswahr fur Wasserschall und Geophysik, Kiel, West Germany)

A general scheme to explain the excess propagation loss in sound channels is outlined, in which the stochastic variability of the environment is combined with the usual deterministic effects on propagation loss. Then a method, previously reported by the author in Acustica [35 (1976)] to handle stochastic sound speed variations in ray tracing routines is applied to model the excess attenuation for the Hudson Bay data. Finally, results will be presented indicating that the excess attenuation in the sound channel is a function of both the variability of the environment and the bottom loss. [Wor:k supported by NAVSEA. 1 
6.ood

9:58

P23. Variability of low-frequency sound absorption in the ocean: $\mathrm{pH}$ dependence. R. H. Mellen and D. G. Browning (Naval Underwater Systems Center, New London Laboratory, New London, CT 06320)

Measured values of absorption coefficients in sea water below $1 \mathrm{kHz}$ show a marked regional dependence; for example, the value in the North Atlantic is found to be about twice that in the North Pacific. Recent laboratory measurements have demonstrated that the absorption mechanism is a boric acidborate relaxation. Since this boric acid reaction acts as a buffer in sea water, the absorption coefficient is strongly $\mathrm{pH}$ dependent. Over the range of $\mathrm{pH}$ values encountered in the oceans (7.5-8.2), the absorption is a monotonically increasing function of $\mathrm{pH}$ and has a range of values of roughly $4: 1$. Comparison of experimental absorption coefficients and predictions based on reported local pH values show reasonably good agreement. [Work supported by NUSC.]
10:02

P24. Source-speed effect on fluctuation time scale. I. J. Rosenbaum (Acoustics Branch, Naval Surface Weapons Center, White Oak Laboratory, Silver Spring, MD 20910)

The time scale of acoustic fluctuations has been found to depend on source speed for a shallow-receiver shallow-sound source configuration in deep water off the coast of St. Croix, U.S. Virgin Islands Measurements were made of a $135-\mathrm{Hz}$ cw source towed at different speeds on a tangential track through a point 7.5 miles from the receiving hydrophones. Higher source speeds consistently gave rise to shorter correlation decay times. As the data integration period was increased from 5 to $100 \mathrm{sec}$, the measured correlation decay times increased as well, covering the range of $4-462 \mathrm{sec}$ for various source-speed-receiver-depth combinations. The position of the receiver with respect to the thermal layer depth also plays a significant role.

\title{
íngy cate \\ Demonstration Paper \\ P25. Approximate ray angle diagram. Henry Cox (Defense Advanced Research Projects Agency)
}

The Ray Angle Diagram presents loci as a function of depth of the angles which selected rays make with the horizontal as sound propagates in the ocean. It provides useful qualitative information about deep ocean propagation and the vertical distribution of signal and ambient noise power. The loci are based on Snell's law. It is shown that an Approximate Ray Angle Diagram (ARAD) can be easily constructed directly from the Sound Velocity Profile since on an appropriate scale the loci of one minus the cosine of the ray angle versus depth are approximately mirror images of the Sound Velocity Profile. The approximations are shown to involve negligible errors for cases of interest in underwater acoustics. The use and construction of the ARAD are illustrated with examples. A simple graphical technique is presented for annotating the ARAD with range information from which rays may be plotted without the use of a computer.

WEDNESDAY, 17 NOVEMBER 1976

COUNCIL/CHAMBER ROOM, 8:30 A.M.

\section{Session Q. Noise III: Airborne Noise Control in Maritime Structures}

\author{
Curtis I. Holmer, Chairperson
}

National Bureau of Standards, Washington, D.C. 20234

\section{Contributed Papers}

$8: 30$

Q1. Noise on dredging ships and auxiliary vessels, R.W. Heymann and F.Z. Sachs (U.S. Army Environmental Hygiene Agency, Aberdeen Proving Ground, MD 21010) and E. P. Fortino (Marine Design Division, U.S. Army Corps of Engineers, Custom House -2d and Chestnut St. , Philadelphia, PA 19106)

High noise aboard dredges and auxiliary vessels is for the most part attributable to diesel engines. Diesels are used in dredges for propulsion, dredging pump operation, and electrical generation. High diesel noise is as a rule confined to the diesel compartments. Other potentially high-noise sources are the ventilation system; and various mechanical equipment such as conventional and hydraulic pumps, motors, and compressors. Sources other than diesel and ventilation systems produce high noise only in their immediate vicinity. With personnel confined to dredges, hearing conservation criteria must account for 24-hour-per-day exposure as well as an 8-hour-per-day workspace exposure. Approaches for noise control depend on whether a ship is being retrofitted or newly designed. Costs are important. For new designs, isolation of diesels and personnel within separate compartments and judicious location of noise-producing equipment with respect to quarters are effective control methods. For existing dredges, personnel boothes are an effective solution. Ventilation noise is reduced with conventional building industry approaches of duct linings and silencers. Sealed passenger compartments and acoustically treated engine compartments are utilized on launches and auxiliary vessels.

$$
8: 40
$$

Q2. Development of the Proposed Canadian Noise-Level Regulations For Vessels Engaged in Towing, K. D. Harford and C.W. Wakefield (Acoustical Englneering, 1727 West 2nd Ave. , Vancouver, BC, Canada V6J. 1H8)

The paper will describe the towboat noise-control experiments, noise-level survey, and general rationale behind the 
Proposed Canadian Noise-Level Regulations for Vessels Engaged in Towing (1975). The proposed regulations are based on what was determined to be the technically feasible noise levels for the large majority of British Columbia vessels at the time of the study with provisions for reduced levels for vessels constructed in the future when marine-noise-control practices will have advanced and become more widely adopted. The relative importance of airborne and structure-borne noise transmission aboard towboats and the significance of this relation to the general philosophy of towboat noise control will be discussed.

$$
8: 50
$$

Q3. Recommended maximum noise and vibration levels on ships. A Schwartz (Dr. A. Schwartz \& Co., Acoustical Consultants, P.O. Box 70:37, Haifa, Israel), P. Munch (Ministry of Transport), and L. Naim (Ministry of Labour)
With the intention to ineorporate it in a revised version of the "Regulations for the Prevention of Harmful Noise on Ships (1972)" a special "Notice to Shipowners and Shipbuilders" (SHN 4/76) was prepared by a technical committee ind issued by the Ministry of Transport, Department of Shipping and Ports. The recommended maximum Noise Rating Levels (NI) for existing ships and for new ships, respectively, are navigating bridge and radio room $65 / 60$, crew cabins $65 / 55$, mess and recreation room $75 / 65$, general machinery spaces $85 / 85$, engine control room $75 / 70$, and engire workshop $80 / 75$. The maximum reconmended acceleration $\mathrm{m} / \mathrm{sec}^{2}$-overall values from 2 to $8000 \mathrm{~Hz}$ ), for vertical and horizontal acceleration. respectively, are: crew cabins, mests and recreation rooms $0.4 / 0.2$, general machinery spaces $\delta .0 / 1.0$ and engine control room $0.6 / 0.3$.

\section{Invited Papers}

9:00

Q4. Airbolne noise control in maritime structures-an overview。 D. L. Nelson (Marine Sciences Department, Bolt Beranek and Newman Inc., Cambridge, MA 02138) and D. ?. Lewis (Naval Ship Engineering Center, Hyattsville, MD 20852)

Similar ities and differ ences of shipboard airborne noise problems and problems of noise in buildings, industrial plants, and transportation systems are discussed. Various types of shipboard environments are considered: these include machinery spaces, living compartments, office, command/control rooms, and deck stations. Criteria reflecting the dif.erent operational requirements are presented. Some special problems unique to ships are uausual noise sources such as active sonar ping and cavitating propellers, close proximity of personnel to major machinery, and ship structure as a sIgnificant sound-transmission pa:h. Methods of noise control are subject to constraints not common to other branches of ayoustics. These include space and weight limitations as related to craft performance, machinery operation, and maintenance. Materials and constructions used in land-based applications may be inappropriate in the maritime environment, because of fire hazards and contamination by oils. fuels, or salt water. The range of current activities in shipboard airborne noise control is outlined and the major deficiencies in the state of the art are discussed.

\section{$9: 25$}

Q5. Analytic techniques for shipboard structure-borne noise control. D. Feit (Ship Ac sustics Department, David W. Taylor Naval Ship Research and Development Center, Carderock, $\mathrm{MD}$ 20084)

Ships like many other modern forms of transportation have become noisier through the years. This is in no small part due to propulsion equipment working at higher horsepowers and pressures and oftentimes packaged more densely into lighter, more efficient structures which can unfortunately be acoustically more transparent. The primary path of sound transmission through ships is via structural waves which propagate throughout the ship's structure. These waves are first generated by the acoustic energy of the machinery source coupling with the adjacent structure or directly via vibrations generated by forces transm tted through the machinery bearings. The ship's structure then provides an efficient transmission medium for sound energy to propagate from one part of the ship to another. The present paper describes the analytical tools that are available to the shipboard noise-control eagineer to deal with the structure-borne noise and vibration isolation design problems.

\section{$9: 50$}

Q6. Overview of recent research-summary of the 1976 International Symposium on Shipboard Acoustics. Miguel C. Junger (Cambridge Acoustical Assoc. , Inc. , 1033 Massachusetts Ave., Cambridge, MA 02138)

Recent Legislation in Europe on permissible noise levels in commereial shipping has heightened concern and increased research efforts to solve noise-control problems. Technisch Physische Dienst TNO-TH, Delft, Netherlands, has organized an international s.. posium of invited speakers on this topic in order to encourage dissemnation of new research results. In this summary of the conference by this participant, some of the more significant research results will be discussed. Results will be selected with regard to their expected significance for influencing the design of new commercial ships or modifications of existing ships. 
Q7. Correlation of predicted versus measured sound levels in naval surface ships. Samuel Feldman (NKF Engineering Association, Inc., 8720 Georgia Ave., Silver Spring, MD 20910)

The U.S. Navy and U.S. Coast Guard have jointly sponsored the development of an airborne noise prediction procedure for military surface ships, which was published in a report entitled "Handbook for Shipboard Airborne Noise Control" dated 13 February 1974. This paper is based on a study aimed at verifying the "Handbook" prediction procedure by comparison with full-scale, shipboard measurements. The U.S. Navy Spruance Class Destroyer (DD 963) offered an excellent opportunity for making such comparison. During the design phase, predictive calculations of sound pressure levels were made for all manned spaces. During the trials period airborne noise measurements were made in these spaces. The paper compares predicted versus measured sound levels in a limited number of spaces and also examines the source sound power level prediction formulae against measured power levels for various types of machinery. On the basis of this one ship sampling, reasonably good correlation was demonstrated between the predicted and measured sound pressure levels.

$$
10: 40
$$

Q8. Noise control in boats and small ships, J. I, Smullin (Marine Sciences Department, Bolt Beranek and Newman Inc., Cambridge, MA)

Noise-control problems in boats and small ships are shown to differ from those of other ship classes due to geometrical scaling laws for person occupied spaces, and by high power to displacement ratios common to them. For the fastest boats, noise sources from propeller cavitation, flow, and spray noise have pronounced importance. Obstacles to noise-reduction techniques outlined are sensitivity of boat performance to weight, expected seaway induced acceleration loads, and corrosive effects of salt environment, Criteria for noise control are presented based on ship function: pleasure/work and continuous running/intermittent operation. Examples illustrating the general problem include measured machinery foundation impedance and vibration mount effectiveness, measured transfer functions for structureborne noise. Examples of noise-control techniques include measured results with lightweight joiner-work partitions, isolation of inter ior trim from hull shell, and successful use of structural damping materials.

$$
11: 05
$$

Q9. Noise-control design problems of air cushion vehicles (ACV) and surface effect ships (SES), M. E. Dvornak (Bell Aerospace Textron, P. O, Box 29:307, New Orleans, LA 70189)

A description of SES and ACV design features which make these craft/ships unique from conventional displacement type ships are presented. The uniqueness of the SES/ACV designs are shown to present new problems relative to ship acoustical design. The impact of the SES/ACV design features on the source-receiver acoustic environment are defined as well as the airborne and structure-borne noise paths between the noise sources and receivers. Examples of test measurements made on SES/ACV type craft are presented to show the contribution of the various noise sources to the total noise at a particular location. The primary noise problem on SES/ACV type craft are shown to be predominately in the lowfrequency region. The noise criteria utilized in the design of SES/ACV's are also examined. Noise-control design approaches which address the various noise sources on SES/ACV'S are presented relative to meeting the established noise criteria. Lastly, SES/ACV noise-control design approaches are compared to those utilized for land-based applications.

$$
11: 30
$$

Q10. Noise exposure and control on fixed marine structures, S.H. Judd (Standard Oil Company of California, 225 Bush St., San Francisco, CA 94104)

Machinery noise sources on drilling and producing platforms include engines, turbines, gears, generators, pumps, and compressors. Noise transmission is both structure borne and airborne. Exposure evaluation requires consideration of work shifts ranging up to 12 hours per day, seven days in a row, as contrasted to the typical on-shore 40 -hour work week. Exposure time is not limited to the work shift for those who must live on the structure. The design problem is to avoid or eliminate excessive noise levels. If this is not feasible, noise levels are minimized both as to intensity and the physical area affected by use of quiet machinery. Enclosure and/or other acoustical treatment is then used to bring exposure within acceptable limits. Off-duty areas and crew quarters are placed in the quietest available location, and isolated from structure-borne and airborne noise. Examples of noise sources and control measures are illustrated by case histories. 


\title{
Session R. Psychological and Physiological Acoustics III: Cochlear Models; Distortions
}

\author{
James T. Marsh, Chairperson \\ School of Medicine, University of California, Los Angeles, California 90024
}

\section{Contributed Papers}

9:00

R1. Permeability of fluid flow through outer hair cell cilia. G. H. Frommer and C.R. Steele (Department of Aeronautics and Astronautics, Stanford University, Stanford, CA 94305)

A $400 \times$ scale model of the reticular lamina with three rows of w-shaped obstacles simulating the outer hair cell cilia bundles was constructed. The pressure required to produce a given low rate of fluid volume flow through the lamina was measured. The results are highly repeatable and show a nonlinear effect with the following properties: (1) A sharply defined threshold, (2) increasing permeability for flow rate from threshold to about $40 \mathrm{~dB}$ over threshold, and (3) asymmetry in permeability. For the 40- $\mathrm{dB}$ range of flow rate above the threshold of nonlinearity, the outward flow from the spiral sulcus to the scala media encounters some $15 \%$ less resistance than the inward flow. Although such a nonlinearity is intriguing, a tentative calculation with physiological parameters places the nonlinear ity threshold at about $80 \mathrm{~dB}$ SPL. So at present, it seems unlikely that the phenomenon is related to any significant cochlear mechanism. [Work supported by NTH. I

\section{$9: 15$}

R2. Comparison of one- and two-dimensional cochlear models. Man Mohan Sondhi (Acoustics Research Department, Bell Laboratories, Murray Hill, NJ 07974)

A technique will be described for solving a two-dimensional cochlear madel proposed by Lesser and Berkley [J. Fluid Mech. 51, 497-512 (1972) $]$ and developed further by Allen [J. Acoust. Soc. Am. 59, S3i (A) (1976)]. It will be shown that the two-dimensional model is well represented by a palr of second-order differential equations. Several functions (e. g. , basilar membrane velocity versus frequency and distance, driving point impedance at the stapes versus frequency, etc.) will be derived from the two-dimensional model and compared to the corresponding funetions derived from the one-dimensional model.

$$
9: 30
$$

R3. Cochlear wave mode transformations, John W. Haggerty (WIBAR Enterprises, 252 South Oxford Ave., Los Angeles, CA 90004)

A three-dimensional "first filter" model, describing the transition from long to short cochlear traveling waves, contains no resonance. Amplitude maxima of middle- and lowfrequency wave envelopes are calculated by this model to occur at the place where longitudinal wavelength equals $4 \pi k b(x)$, where $b(x)$ is the local width of the basilar membrane and $k$ is the local (near unity) ratio of the longitudinal and lateral (dissipative) traveling-wave propagation velocities. These two wave modes share the same potential function and are thus tightly coupled; their phase relationship is established by both the curvature of the cochlea and the stiffening effects of the Arches of Corti near inner radius. The velocity-versus-boundary dimension methods used in constructing the above modeling are known collectively as normal-mode analysis. Normal-mode analysis also suggests many reasonable "second-filter" modes of vibration possibly operating within the Organ of Corti (which gelled material supports the bases of all hair cells). At high frequeney, a vibration-absorber wave mode (squeeze of the Organ of Corti) can amplify the displacement of the traveling wave, especially at the inner hair cells, while simultaneously extinguishing (by well known force-balance processes) that traveling wave on the basilar membrane. At lower frequencies, where the Organ of Corti spans relatively less of the basilar membrane width, some constrained-sloshing wave modes in the gelled material also preferentially excite inner hair cells. Close agreenent wil.h published hylrodynamic cochlear wave observations is shown. Confirmation of any of these distinct (but theor etical) wave modes within the Organ of Corti awaits measurement of directional propagation velocities and elastic properties throngh the gelled material as it lies flooded and attached to the basilar membrane.

\section{$9: 45$}

R4. Cochlear nonlinearity and second filter: psychophysical estimation of model parameters. H. Duifhuis (Institute for Perception Research IPO, Den Dolech 2, Eindhoven, The Netherlands)

Recently we proposed a theoretical model for cochlear nonlinearity and second filter [J. Acoust. Soc. Am. 59, 408-42;3 (1971)l. From psychophysical two-tone suppression data we attempted to estimate characteristics of the two filters and of the compressive nonlinearity $y=x^{\nu}$. In our first exper iments probe frequency (suppressee as well as test probe) was $1 \mathrm{kHz}$. Masker (suppressor) frequency was a parameter. We used the pulsation-threshold method, where misker plus suppressee and test probe alternated. The pulsation threshold of the test probe was measured as a function of masker level, suppressee level being a fixed parameter. Data curves typically are characterized by a horizontal part, a descending part, and an ascending part. The descending part, reflecting suppression contains the wanted information. Insofar as our interpretation of the data is valid, we estimate for $\nu$ a value from 0.25 to 0.8 , depending on masker frequency. The low-frequency par1 of the second-filter characteristic levels off at approximately 10- $\mathrm{dB}$ attenuation. The first-filter character istic depends on level. Thus, the data show more nonlinear ity than the model accounts for. Possible modifications will be discussed.

\section{0:00}

R5. The critical band; its relation to cochlear nonlinear ity and second filter. H. Duifhuis and W. F. Simons (Institute for Perception Research IPO, Den Dolech 2, Eindhoven, The Netherlands)

The measurement of the effect of the bandwidth of a noise masker which masks a centrally located tonal probe, provides a possibility to determine the critical bandwidth. The result of such a measurement typically shows two types of asymptotic behavior: For narrow bands the masking effect is determined by the power in the band, for wide bands, hovever, by the power density. The intersection of the asymptotes defines the critical band. If we assume that the masking effect is determined by the signal-to-noise ratio of activity in the probe channel the above results can be predicted by the model on cochle:r nonlinearity and second filter [H. Duifhuis, J. Acoust. Soc. Am. 59, 408-423 (1976) ]. The predicled critical bandwidth then depends relatively strongly on the tuning disparity between first and seoond filter and relatively weakly on filter shapes. Also we predict certain observed differences between 
nonsimultaneous and simultaneous masking for the above stimuli.

$$
10: 15
$$

R6. Model study of nonlinear response to palred clicks. J. L. Hall (Bell Laboratories, Murray Hill, NJ) and M. R. Schroeder (Drittes Physikalisches Institut, Universität Göttingen. Federal Republic of Germany and Bell Laboratories, Murray Hill, NJ)

T. J. Goblick, Jr. and R. R. Pfeiffer [J. Acoust. Soc. Am. 46, 924-938 (1969)] found various nonlinear effects in auditorynerve responses of cats to paired clicks. We have been able to reproduce these effects in a nonlinear model of the basilar membrane [M. R. Schroeder, Proc. IEEE 63 (9) , 1332-1350 (1975) ; J. L. Hall, J. Acoust. Soc. Am. 56, 1818-1828 (1974)] in greater detail than has been possible in previous studies [D. O. Kim, C.E. Molnar, and R. R. Pfeiffer, J. Acoust. Soc. Am. 54, 1517-1524 (1973)]. To investigate the origin of these nonlinear effects, selected portions of the membrane model were made linear. In this manner it was found that the effects arise in two regions of the membrane model. The strongest contribution occurs just basal to the place of observation (characteristic frequency $f=f d$ ). A weaker contribution occurs just basal to the place with characteristic frequency $f=2 f_{c}$.

\section{$10: 30$}

R7. Nonexponential integrator in an energy-detection model for auditory perception. J. L. Hall and Man Mohan Sondhi* (Acoustics Research Department, Bell Laboratories, Murray Hill, NJ 07974)

In a continuation of a study described at the 88th Meeting of the Acoustical Society of America [M. M. Sondhi and J. L. Hall, J. Acoust. Soc. Am. 56, S37 (1974) l, the absolute threshold for two-component tone bursts of $500-\mathrm{msec}$ and 1sec duration was measured as a function of the frequency spacing between the two components. The average frequency of the two components was $700 \mathrm{~Hz}$, and the frequency difference between the two components ranged from 0 to $8 \mathrm{~Hz}$. When interpreted in terms of an energy-detection model (peripheral filter followed by squarer, integrator, and threshold detector), the measurements give an estimate of the window shape of the integrator. Experimental results are fit poorly with an exponential window. A better fit is obtained with a window decreasing initially more rapidly than exponentially. A nonexponential window is also suggested by psychophysical data on detection of pulse trains [J. Zwislocki, J. Acoust. Soc. Am. 32, 1046-1060 (1960)]. *The order of names was decided by tossing a coin.

\section{$10: 45$}

R8. Recursively generated combination tones. Shelley J. Russek and Donald I. A. MacLeod (Department of Psychology, University of California at San Diego, La Jolla, CA 92093)

When a presented stlmulus includes three pure-tone primaries of frequencies $f_{1}, f_{2}$, and $f_{3}$, a combination tone generated by two of the primaries may combine with the third primary in generating further combination tones: for instance, $2 f_{1}-\left(2 f_{2}-f_{3}\right)=2 f_{1}-2 f_{2}+f_{3}$. If the parent combination tone (in this case, $2 f_{2}-f_{3}$ ) is eliminated by canceling it with a pure tone of appropriate frequency, the "second-generation" tone $\left(2 f_{1}-f_{2}+f_{3}\right)$ disappears along with it. The process of combination-tone generation thus appears to be a recursive one in which a combination tone, once generated, may particlpate in the generation of other combination tones. This suggests that distortion products in the ear are coupled back to the input of the distorting stage and themselves undergo distortion. Similar experiments using only two primaries suggest that all combination tones of odd order arise mainly from the recursive generation of cubic distortion products. The combination tone $2 f_{2}-f_{1}$, as well as the tone $2 f_{1}-f_{2}$ may be an important contributor to higher-order tones.

\section{$11: 00$}

R9. Masking effects at cubic intermodulation product frequencies, John Erdreich (Department of Otorhinolaryngology, The University of Oklahoma Health Sciences Center, OkIahoma City, OK 73190)

When in the presence of two monaural phase synchronized tones $f_{h}$ and $f_{1}$ a third tone of frequency $2 r_{1}+f_{h}$ or $2 f_{1}-f_{h}$ (a pair of intermodulation distortion products) is varied in phase relative to itself, the amplitude to which the tone must be raised to exceed threshold changes. These phase-related masked threshold shifts may be attributable to the time structure of the tones, to interaction between nonlinear distortion products and the maskee tone, or to changes in the detection criterion for the maskee. The form of this monaural phase effect may be either sinusoidal and of a single period, or it may exhibit a double peak. The single-peak monaural phase effect is produced with a maskee of $2 f_{1}-f_{h}$ if the distortion product is made inaudible by either narrow-band masking centered at $2 f_{1}-f_{h}$ or by reducing the level of the primaries and therefore the level of the intermodulation product. With a maskee of $2 f_{1}+f_{h}$ the phase effect is sinusoidal until the distortion product exceeds threshold levels. This suggests that the double-peak form of the monaural phase effect is the result of suprathreshold ambiguities of vector summation between maskee and the intermodulation distortion product. Furthermore, the demonstration of phase effects with added narrow-band noise indicates that the sinusoidal threshold effects with long-duration (greater than the maskee period) maskee tones is not a function of fine time structure [E. Zwicker, Acustica 34, 138-146 (1976) ] but of vector summation between distortion product and maskee.

\section{$11: 15$}

R10. Nonlinear distortion in listeners with sensorineural hearing loss. R. Lindstrom and B. Leshowitz (Department of Psychology, Arizona State University, Tempe, AZ 85281)

Evidence suggesting that combination tones are not a result of middle-ear or basilar-membrane mechanics has been presented in the literature. It has been proposed that the sensory cells in the cochlea are the site of the site of the nonlinearity. However, the precise location of the sensory region responsible for the generation of the combination tones is not known. This important theoretical problem was explored using a tonal masking paradigm with listeners having a localized region of sensor ineural hearing loss. Psychoacoustical tuning curves were measured in order to delineate the frequency region of combination tone audibility. By manipulating the frequency of the flxed probe with respect to the listener's hearing loss, the sensory region over which the nonlinear distortion product is generated was determined. Results of this study showed that when the fixed probe was just lower in frequency than the region of the threshold elevation and $F_{m}<F_{p}$, combination tones were not detected. However, with the probe just higher in frequency than the region of threshold elevation, audible combination tones were produced. The conclusion was drawn that the nonlinearity resides in the cochlear region basal to that of the primaries.

\section{$11: 30$}

R11. Time dependence of pitch perception-pitch step experiment. W. M. Hartmann and B. J. Blumenstock (Physics Department, Michigan State Univergity, E. Lansing, MI 48824)

In the pitch step experiment subjects tune an oscillator in three successive adjustments to match the pitch of a $15-\mathrm{msec}$ sine-wave tone of frequency $f_{2}$ that is immediately preceded by a 4-sec sine-wave tone with frequency $f_{1}\left(900<f_{1}<1000 \mathrm{~Hz}\right)$ of the same intensity $(92 \mathrm{~dB})$. We find that for upward steps $\left(f_{2}>f_{1}\right)$ the matched tone frequency $p_{2}$ tends to be greater than $f_{2}$. This effect, whereby the pitch of tone 1 repels the pitch of tone 2 increases for decreasing $\left|f_{2}-f_{1}\right|$. The effect is reminiscent of the repulsion of a tone by a narrow noise band. [J. C. Webster and D. R. Muerdter, J. Acoust. Soc. Am. 37, 382- 
383 (1965) 1. Therefore, a plot of $p_{2}-f_{1}$ versus $f_{2}-f_{1}$ is nonlinear (and possibly discontinuous) near the origin. If $p_{2}$ is regarded as a description of the state of a pitch perceptor 15 msec after the step then the nonlinearity guarantees that no linear differential equation can describe the time-dependent response of the pitch perceptor. Dichotic experiments with tones 1 and 2 in different ears suggest: that a repulsive-type nonlinear ity is still present, though reduced in size. For downward pitch steps two of our subjects exhibit a repulsive effect $\left(p_{2}<f_{2}\right)$ and two subjects produce anomalously high $\rho_{2}$ tunings.

\title{
Session S. Musical Acoustics I: Music Perception and Production
}

\author{
P. L. Divenyi, Chairperson \\ Veterans Administration Hospital, Martinez, California 94553
}

Contributed Papers

9:00

S1. Subdivision of the beat: Estimation and production of time ratio by skilled musicians. Ronald L. Knoll and Saul Sternberg (Bell Laboratories, Murray Hill, NJ 07974) and Paul Zukofsky (Center for Contemporary Performance Practice, Port Jefferson, NY 11777)

Professional musicians served as subjects in experiments on time estimation and production. In one procedure, equally spaced high-frequency elicks defined rhythmic beats. Intervening lower-frequency marker clicks indicated a fraction of the interval between beats. Subjects estimated the fraction. In a second procedure, equally spaced clicks were presented without the intervening marker clicks. Subjects attempted to produce a specified fraction of the interral between beats by responding with a finger tap after each click. If produced fractions are adjusted by estimating the time between elick occurrence and response feedback, then any biases in the two tasks should be equal in magnitude and opposite in direction. For large fractions, estimated and produced fractions were unbiased. However, for small fractions, both estimated and produced fractions were too large. With respect to a feedback theory of production, the common direction of the two biases is paradoxical. [Work partially supported by IRCAM and Rockefeller Foundation. J

\section{9:15}

S2. Octave convergence in melodic perception. Wendy $\mathbf{L}$. Idson and Dominic W. Massaro (Department of Psychology, University of Wisconsin, Madison, WI 53706)

Previous research indicates that the pitch of a single tone can be conceptualized as a bidimensional quantity, reflecting the overall pitch level of the tone (tone height) and its position in the octave (tone chroma). It has been suggested that the dimension of tone chroma is irrelevant to perception of the component tones of a melody. Supporting this argument, perception of a melody is disrupted when its tones are displaced by octave multiples. This has been interpreted as showing that melodic perception is based upon the magnitude of successive pitch intervals. However, octave displacement violates both interval magnitude and direction. In the current study, melodies were subjected to structural transformations in which the tones were displaced by octave multiples, either preserving or violating the direction of successive pitch intervals (melodic contour). Replicating previous research, when melodic contour was violated performance was severely disrupted. When contour was preserved, however, the melodies were identified as accurately when the tones were displaced by octave multiples as when the melodies were untransformed. These results demonstrate that the bidimensional mcdel of pitch perception applies to melodies, and that contour plays a significant role in melodic perception. [Work supported by NIMH.]

$$
9: 30
$$

S3. Dis ruption of categorical processing in music. J.A. Siegel (Department of Psychology, University' of Western Ontario, London, Ontario, Canada N6A 3K7)

Musicians judged 13 tonal intervals ander magnitude estimation instructions. The intervals were spaced in 20 -cent steps and covered a range of three semitone categories. In experiment I, the subjects were naive with respect to the exact nature of the stimulus set, and under these conditions, evidence for categorical perception was obtained. The psychometric functions were well predicted by these subjects' identification of the same stimuli. Stimuli ir: different categories were judged as perceptually distinct, tut the judgment of intonation within a eategory was poor. That is, these subjects were unable to distinguish among shar], in-tune, and flat stimuli with the same interval name. In Experiment II, subjects were given specific information concerning the nature of the stimuli. Their judgment of within-category differences failed to improve, and their assignmert of the stimuli to the three interval categories became mort variable. It appears that musicians are poor at judging witt in-category differences in intonation, and that attempting to do so disrupts the processing of categorical information.

9:45

S4. Temperament of the musical seale in fixed pitch instruments: A contemporary psychological śdjustment to environment. George W. Mulder (Northwestern Michigan College, Traverse City, MI 49684)

The relation between just intonation and equal temperament has been a subject of concern for both musician and physicist. The Stevin monochord in 1596 seems to have been the first solution in Western thought. However, Tsai-yu's Monochord in 1595 warrants attention in Eastern thought. As with Vicentino's solution in 1555, aesthetic considerations, tentative though they may be must be regarded. Through measurement and examined response, psychological adjustments to temperament are observed and recorded. The :esults of these perceptions qualifies the listener's attitude toward temperament of 
the musical scale. Environment and the status quo of aesthetic standard is observed the paramount determinant.

10:00

S5. Perception of musical-interval tuning. Donald E. Hall (Physics Department, California State University, Sacramento, CA 95819)

When two musical tones are heard together, there is a perception of whether or not they are "appropriately" spaced in pitch. This judgment is dependent on musical Lraining and/or aptitude, as well as on several physical variables. Terminology must be used with care, because such words as "consonant," "dissonant," or "fused" already have other meanings. It would be of real interest to musicians to establish more quantitative information about tuning perception. Some experiments currently in progress will be briefly deseribed.

\section{0:15}

S6. The paradox of pitch categories. W. Siegel (Department of Psychology, University of Western Ontario, London, Ontario, Canada N6A 3K7)

The categorical perception of pitch by musicians [e.g., W. Siegel and R. Sopo, J. Acoust. Soc. Am. 57, 511 (1975) j raises an interesting question: How can they tune their instruments when they cannot tell sharp from flat? Here, the results of two experiments on ELP, a musician with very fine absolute pitch, suggest a resolution of the categorization paradox. In a production task, which emulates tuning an instrument, he was very accurate at adjusting the frequency of an oscillator to a value corresponding to four musical notes, $\mathrm{g} \#$ $(415 \mathrm{~Hz}), \mathrm{a}(440), c(523)$, and $d \#(622)$. However, in an identification task involving listening to single brief notes, he show'ed striking evidence of categorical perception. That is, he judged $400-, 500-$, and $600-\mathrm{Hz}$ tones to be accurate examples of the notes $g \#$ (415), $c(523)$, and $d \#$ (622) on most of the trials.

S7. Recognition of musical triad quality. Rosemary N. Killam (Department of Nusic, University of Delaware, Newark, DE 19711) and Paul V. Lorton and Earl D. Schubert (Stanford University, Stanford, CA 94305)

Certain patterns of pitch, such as chords and scales, are central to the organization of traditional music. These patterns are identified by standardized nomenclature, and form a basis for analytical listening to music. The auditory processes involved in recognition may be revealed by comparing the ability of trained subjects to categorize these standard patterns. Perceptual confusion among standard, three-tone stimuli was studied in 19 musically trained subjects. A solid-state organ, interfaced to a digital computer, was used to present the stimuli; a terminal connected to the same computer was used to collect the responses. All subjects received the same stimuli, randomly accessed by the computer program. The subject indicated whether the signal presented was major, minor, augmented, or diminished. The three-note signals were presented in three manners: simultaneously, ascending (in pitch), and descending. All stimuli were presented at both 0. 2- and 0.1-sec note duration. Accuracy was least for shorter duration and for simultaneous presentation. Different triad qualitites maintained essentially the same performance rank through the different modes of presentation. Patterns of confusion and response bias suggest the subjects' use of preexistent auditory images.

\section{0:45}

S8. Time dependence of pitch perception-vibrato experiment. W. M. Hartmann and K. A. Long (Physics Department, Michigan State University, East Lansing, MI 48824)

Subjects listened to diotic sine-wave tones $(72 \mathrm{~dB})$ with center frequency $f_{0}\left(700 \leq f_{0}<900 \mathrm{~Hz}\right)$ frequency modulated at subaudible rate $v(0.5<v 10 \mathrm{~Hz})$. In a 2 IFC experiment subjects decided whether the first or second tone of a pair of tones had the wider vibrato. One tone of the pair was made with sinewave FM waveform; the other wias made with triangle FM waveform. The sine-wave modulation excursion was fixed at $\Delta f_{s}= \pm 20 \mathrm{~Hz}$; the triangle wave excursion varied amongst the trials $0.8 \Delta f_{s}<\Delta f_{T}<1.5 \Delta f_{s}$. (In this range of $v$ and $\Delta f$ it is impossible to identify the FM waveform.) The subject judgments were analyzed by a least-squares procedure to determine a locus of subjective equality, $\Delta f_{T}\left(v^{\prime}\right)$, for which vibrato widths due to the different modulations seem to be equal. The resulting locus unambiguously show's that pitch averaging takes place for such stimuli. For $v<7$ IIz the locus of subjective equality can be reproduced by a model of pitch averaging with averaging-time constant of $130 \mathrm{msec}$, a factor of 10 greater than pitch recognition times. For $v>7 \mathrm{~Hz}$ a different averaging process appears to proceed. This simple model was tested by additional identical experiments $w$ ith $\Delta f_{s}- \pm 40 \mathrm{~Hz}$ and $\Delta f_{s}$ $= \pm 10 \mathrm{~Hz}$. The model may survive these tests, but in the small $v$ regime we find that the acernging time is approximately inversely proportional to $\Delta f_{s}$.

\section{1:00}

S9. Scaling the perceived $u$ 'idth of vibrato tones. John I. Shonle (Departments of Physies and Psychology, University of Colorado at Denver, Denver, CO 80202) and William R. Simmons (Department of Physics, Lniversity of Colorado at Denver, Denver, CO 80202)

We have investigated the psychological magnitude of the width of vibrato tones. In the experiments reported here, the signal was a modulated $440-\mathrm{Hz}$ sine wave. The method of magnitude estimation was used with 14 subjects, who heard the vibrato tones produced by a speaker in a room, resulting in a power-law exponent of 0.80 . Then eight subjects using the method of magnitude production and listening with headphones set widths for both "twice as wide" and " half as wide," yielding an exponent of 1.85 . The third experiment investigated whether the difference was attributable to differences in psychophysical method or in method of presentation. Five subjects performed magnitude estimation listening with headphones, with a resultant exponent of 1.70 . An experiment on the detection of the presence of vibrato confirmed that there is a significant difference between perceiving vibrato with headphones and from a speaker in a room. Sixteen subjects in a room were performing in a $2 \mathrm{AFC}$ task at the $83 \%$ correct level at a width of \pm 2 cent, whereas ten subjects with headphones were able to score only $68 \%$ correct at a width of \pm 10 cent.

\section{1:15}

510. Perception and production of double stops in violin playing. Paul Zukofsky (Center for Contemporary Performance Practice, Port Jefferson, NY 11777) and Joan E. Miller and J. Kohut (Bell Laboratories, Murray Hill, MJ 07974)

The playing of double stops (two notes produced simuItaneously) was investigated to determine differences in reaction times and to consider the implications regarding the player's organization of information. Using a battery powered instrument equipped with metal strips placed at half-step intervals along the finger board, the violinist's performance was monitored by computer. Signals resulting from contact of the strings with the strips were sampled every $2 \mu$ sec to determine when which pitches were played. The player responded to stimuli consisting of double stops, single pairs of double stops and alternating pairs presented on a cathode-ray tube in conventional music notation. Reaction times were used as measures of ease in playing, for estimating perception versus motor times, and for examining the additivity of the processes involved. Preliminary findings suggest that (1) timings for single pairs depend upon the order of the members of the pair, and (2) the rank order of timings for single pairs correlates poorly with that of average speed for alternating pairs. The pedagogical significance will be discussed. [Work partially supported by IRCAM and the Rockefeller Foundation.] 
$11: 30$

S11. Synthesis of chime tones. Harvey Fletcher and Irvin G. Bassett (Department of Physies and Astronomy, Brigham Young University, Provo, UT 84602)

Tones from three chimes were recorded and analyzed using a fast-Fourier-transform program. Three-dimensional, perspective plots were made of the sounds in order to determine the salient features. The analysis agrees well with that done previously by other investigators. Synthetic tones were produced by adding a series of sine waves with frequencies, amplitudes, and decay rates taken from the analysis. Listening tests were performed to evaluate the synthesis. Twenty subjects were presented a series of 30 tones consisting of three real and three synthetic sounds repeated five times in a random sequence. The subjects were asked to decide whether each tone seemed real or synthetic. Overall results for the 20 subjects were $55 \%$ right, just slightly above the level of guessing.

$11: 45$

S12. Preliminary analysis of vocal chant. John Large (Department of Music, University of California, San Diego, CA 92161) and Thomas Murry (Veteran's Administration Hospital, San Diego, CA 92161)

The use of chanting as a mode of voice production has been gencrally limited to religious ceremonies, theatrical performances, or classroom exercises such as memorizing the multiplication tables. It has been suggested that chanting of the type done by Tibetian lamas resembles the production of vocal fry. The purpose of this study was to compare vocal chant, vocal fry, and normal phonation on the parameters of mean air-flow rate, fundamental frequency of spectrum. Three males and two females produced samples of vocal chant, voeal fry, and normal phonation. The samples were produced in pairs (i.e., chant normal) with no break between the pairs. The subjects produced two sets of samples, one in a soundtreated room and one in a respiratory mask. The flrst set was used to obtain the acoustic measures; the second to obtain the air-flow values. The results indicate that vocal chant, vocal fry, and normal phonation are produced at three different ranges of air-flow rate. It was also possible to differentiate vocal fry and voeal chant on the basis of voice fundamental frequency.

\title{
Session T. Speech Communication III: Linguistic Phonetics; Vowels; Tone; Miscellaneous
}

\author{
Ilse Lehiste, Chairperson
}

Department of Linguistics, Ohio State University, Columbus, Ohio 43210

\section{Contributed Papers}

9:00

T1. Speech development in infants-vowel production. $P$. Lieberman, R. Buhr, P. Keating, S. V. Hamby, and K. H. Landahl (Department of Linguistics, Box E, Brown University, Providence, RI 02912)

Five infants ranging in age from 12 to 66 weeks at the start of the project have been recorded at biweekly intervals during half-hour play sessions with their mothers. Fundamental and formant frequencies were derived from sound spectrograms, Fourier and Linear Predictive analysis. The data reveal differences in the infants' phonetic development as they gradually acquire the vowels of English. Their formant frequency patterns are outside the range reported by Peterson and Barney (1952) for older children but are consistent with the shorter supralaryngeal vocal tracts of these infants. The formant patterns that specify particular vowels change as the infants mature. $F_{1}$ of $/ \mathrm{ae} /$, for example, changes from 1.0 to 0.7 $\mathrm{kHz}$ for one infant between 37 and 56 weeks. The infants' vowel production and their mothers' responses are consistent with the presence of an innate vocal tract length normalization perceptual mechanism that enters into the development of speech procuction.

$$
\text { 9:10 }
$$

T2. Vowel utterances of young infants. R. E. Stark, J.M. Heinz, and C. Wright-Wilson (Department of Otolaryngology, Johns Hopkins School of Medicine, Baltimore, MD)

Syllable-like utterances produced by 14 different infants aged 1-40 weeks were presented to two trained listeners. The listeners first transcribed a portion of the data phonetically. They then compared transcriptions, at'empted to resolve disagreements and decided upon conventicns to be used. One hundred and twenty-two additional segrients were then transcribed by the listeners independently. On $71 \%$ of these utterances the listeners disagreed in their transcriptions of syllabic nuclei. The remaining $29 \%$, upon which they agreed, were all transcribed as mid or low front. Their judgments, where they disagreed, were always vowels adjacent to one another within the vowel triangle and were also predoninantly heard as mid or low front vowels. The vowels at the extremes of the triangle, $/ \mathrm{a} / \mathrm{,} / \mathrm{u} /$, and $/ \mathrm{i} /$ were not judged to be present at all. Ten vowels upon which the judges agreed and ten upon which they disagreed were analyzed by conventional spectrographic and by computer-assisted spectral teckniques. The results of these analyses will be discussed. [Work supported by NINCDS.]

$$
9: 20
$$

T3. Imitation of synthesized vowels by children and adults. R. D. Kent (Department of Communicative Disorders, University of Wisconsin, Madison, WI 53706)

Imitations of 15 synthesized vowels, some English vowels and some not, were obtained from ten adults (five men and five women) and ten six-year-old children (five boys and five girls). Estimates of the first three formant frequencies, F1, $F 2$, and F3, were made from spectrograms of the vowel imitations. The reliability of reproduction was assessed by calculating standard deviations for five imitations each of ten of the synthesized vowels. Generally, koth the intra- and intersubject variabilities of the formant: frequencies were 
-for children than adults, but the differences in intravariability were not much greater than the differentes in measurement error associated with different fundamental frequencies. Subjects tended to reproduce non-English vowels less reliably than English vowels, although adults wereinflutenced less by phonetic familiarity than were the chifdren. Vowel familiarity appeared to be especially important for reliable reproduction of the $\mathrm{F} 2$ frequency. Plotting of the data for English vowels in a F1-F2 plane with linear dimensions revealed an almost linear clustering for the four subject groups, but the group clustering was not as linear for the nonEnglish vowels. [Work supported by NIH. I

$$
9: 30
$$

T4. Vowel spaces in two Kwa languages. Mona Lindau, Patricia Wood, Philippe LaFage (Phonetics Laboratory, Linguistics Department, University of California, Los Angeles, CA 90024)

The acoustic spaces of two languages with seven vowel systems were compared. The languages were Yoruba and Ewe, both of the Kwa group of Niger-Congo languages. The vowels were/ief aoou/. The formant spaces were based on the production of several token of each vowel by four speakers of each language. While the seven vowels of Ewe are distributed in a highly regular fashion around thervowel space, this is not found in Yoruba, where the upper mid /e/ and /o/ are very close to the high $/ \mathrm{i} /$ and $/ \mathrm{u}$, respectively: The phonetic differences between the two $\mathrm{q}$ af gltages cannot be accounted for by any current theory of the utilization of the vowel space. The results indicate that the historical development of vowel systems is not only influenced by principles of maximal perceptual distance, or "base of articulation," but may also be related to different phonological processes in different languages.

$$
9: 40
$$

T5. Effects ofĩcoarticulation, syllable type, and stress on acoustic French vowel targets. G. F. Chollet (Phonetics Research Facility, University of California, Santa Barbara, CA 93106)

Modern computer speech-signal analy \&its techniques are applied to the study of the acoustic characteristics of surreptitiously recorded conversational speech. Vowel formant values are found to vary considerably more than previous studies have led us to believe. Several parameters that could be causing these fluctuations are dealt with here. The phonemic context appears to be an important cause of variation. A tendency to produce an open vowel in closed syllables would appear to be true for all French yowels, although slightly more pronounced for midvowels. Vowels in the last syllable of a breath group (the stressed syllable in French) are generally more stable than those occurring in unstressed ones. The phenomenon of vowel reduction in French, although less pronounced than in English, is nevertheless quite noticeable. Other possible causes of variation are discussed as well.

\section{$9: 50$}

T6. Control of vocal tract length in speech. Carol Riordan (Phonology Laboratory, University of California, Berkeley, CA 94720)

Accurate control of vocal tract length is essential for the correct production of vowels. Vertical larynx movement and lip spreading/protrusion, often acting together, are important determinants of vocal tract length. The present study was designed to determine whether constraining lip spreading/ protrusion will induce compensatory vertical larynx displacements, particularly on rounded vowels. Upper lip and larynx movement were monitored photoelectrically while French and Mandarin native speakers produced the vowels / $, y, u$ / first under normal speech conditions and then with lip activity constrained. In agreement with the findings of Sundberg (1969), Benguerel (1974), and Ewan (1976), there were significant differences during normal speech in upper lip protrusion and larynx position as a function of the vowel and tone uttered, in particular a difference in lip protrusion between $/ u /$ and $/ y /$. Results further reveal that the generally low Iarynx position of rounded vowels becomes even lower than lip protrusion is constrained. This data has implications for the role of a contrast-preserving strategy in speech production. [Supported in part by the National Science Foundation. I A1so, Department of Langu ges and Linguistics, University df Essex, Colchester, England.

\section{0:00}

T7. Explaining the intrinsic pitch of vowels. John J. Ohala (Phonology Laboratory, Department of Linguistics, University of California, Berkeley, CA 94720) and Brian W. Euldis: (Computer Center, University of Texas, Austin TX 787i2)

The reason why, other things being equal, the pitch of vowels correlates with vowel "height," has long been a subject of speculation. The effect is usually attributed either to acoustic coupling between vocal tract and vocal cords or to the pull of the tongue on the vocal cords. To determine which of these hypothesized factors responsible we observed the effect on the pitch of vowels then ne of these factors (impe-

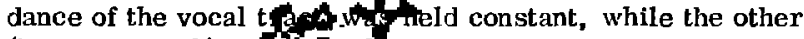
(tongue stretch) wastratied over an exaggerated range, viz., by having eight speakers ther special test utterances when their jaw was propped open by small wooden blocks $\leqslant 10 \mathrm{~mm}$ thick. (Lindblom and Sundberg have shown that under such conditions apeakers can achieve acoustically normal vowels by increasing the distortion-and presumably the stretch-of the tongue body with respect to the jaw and larynx-hyoid complex.) We found a small but consistent increase in the pitch interval between high ald low vowels 4 hen subjects spoke with proppedopen jaw, thus su porting the "tongue-pull" hypothesis. [Supported mosp.]

\section{0:10}

T8. Intensity and duration as correlates of $F_{0}$. Eric Zee and Jean-Marie Hombert (Phonetics Laboratory, Linguistics Department, University of California at Los Angeles, CA 90024)

An experiment was conducted to determine whether intensity and duration are correlated with tonal differences. Taiwanese was chosen because it has five contrastive tones, which are high, mid, low, rising, and falling. In this dialect the syllable [si] occurs on all five tones. Preliminary results reveal that (1) there is a direct correlation between $F_{0}$ and both duration and intensity; (2) falling tone has higher intensity and shorter duration than rising tone; (3) the rate of change of $F_{0}$ is greater in falling tone than in rising tone; and (4) both in falling and rising tones, the rate of change of $F_{0}$ is greater in the second balf $f^{+}$of the entire duration than in the first half. These results are discussed in terms of possible physiological explanations, such as the use of a higher subglottal pressure to produce a higher $F_{0}$. [Research supported by NSF.]

\section{0:20}

T9. Difficulty of producing different $F_{0}$ in speech. Jean-Marie Hombert (Phonetics Laboratory, Linguistics Department, University of California, Los Angeles, CA 90024)

Two studies (Ohala and Ewan, 1973; Sundberg, 1973) have revealed interesting articulatory constraints on the production of certain $F_{0}$ contours. One finding is that it takes longer to produce a rising $F_{0}$ contour than a falling contour covering the same frequency range. The purpose of this paper is to investigate in more detail the articulatory constraints involved in the production of some typical $F_{0}$ patterns found in tone languages. Ten subjects (five speakers of tone languages and five speakers of nontonal languages) were asked to imitate a total of nine fundamental frequency patterns: three levels $(110 \mathrm{~Hz}$, $130 \mathrm{~Hz}$, and $150 \mathrm{~Hz})$ and six contours $(110-130 \mathrm{~Hz}, 130-150$ $\mathrm{Hz}, 110-150 \mathrm{~Hz}, 150-1 \mathrm{~B} 0 \mathrm{~Hz}, 130-110 \mathrm{~Hz}$, and $150-110 \mathrm{~Hz}$ ). In order to control for the effect of vowel quality, three vowels were used: [i], [a], and [u]. Subject's accuracy at matching the various stimuli (in $F_{0}$ and in duration) was taken 
as reflecting the difficulty involved in the imitation of these $F_{0}$ patterns. The results suggest a greater difficulty for rising (versus fallthg) and for higher (versus lower) $F_{0}$ patterns. [Research supported by NSF.]

\section{$10: 30$}

T10. Some perceptual experiments on the Mandarin tones. Victor W. Zue (Research Laboratory of Electronics, Massachusetts Institute of Technology, Cambridge, Mass. 02139)

A series of experiments, using both natural and synthetic speech materials, were conducted to investigate the perception of the four different tones in Standard Peking Mandarin. In the initial experiment, all stimuli were of the same duration and differ only in the fundamental frequency $\left(F_{0}\right)$ contour. It was found that the "averaged $F_{0}$ contours of these tones possess some essential features for absolute identification. It was also found that the frequency range spanned by the four $F_{0}$ contours can be reduced by an order of magnitude without seriously impairing performance. In an extention of the above experiment, the intrinsic differences in duration among the four tones were taken into account, and native as well as naive subjects were used. The remarkable performance of these experiments has led us into an investigation of the categorical boundaries in the perception of some simple $F_{0}$ contours. Some preliminary results of these experiments $w$ ill also be presented. [Work supported by NINCDS. I

\section{$10: 40$}

T11. Tone spreading and perception. Ian Maddieson (Phonetics Laboratory, Linguisties Department, University of California, Los Angeles, CA 90024)

An experiment was designed to determined if perceptual processes contribute to the explanation of asymmetries in "tone spreading" in tone languages. The basic stimulus was a synthesized trisyllabic utterance [wawawal, whose fundamental frequency varied in the following manner. Each stimulus type contained two sections, one with a higher and the other with a lower $F_{0}$. An abrupt change was located at 16 points 15 msec apart ranging from the beginning to the end of the middle syllable. In one series, $\mathrm{H}-\mathrm{V}-\mathrm{L}$, the higher section occurred first, in the other, $\mathrm{L}-\mathrm{V}-\mathrm{H}$, the lower section occurred first. Subjects were presented a randomized sequence of the stimuli from both series and asked to respond "first" or "last" according to whether the middle syllable sounded the same in pitch as the first or the last syllable. To elicit a "first" response, the portion of the middle syllable identical in $F_{0}$ to the first syllable needed to be shorter for the H-V-L series than for the L-V-H series. This result demonstrates a perceptual phenomenon which possibly serves as the basis for the observation that high tones are more likely to spread than low tones. [Research supported by NSF.]

\section{0:50}

T12. Auditory basis of progressive tone spreading. Hector Javkin (Phonology Laboratory, Department of Linguistics, University California, Berkeley, CA 94720)

Relatively low frequencies (which include the $F_{0}$ of human speakers) are believed by many investigators to be processed by a different mechanism than most of the frequencies involved in speech segments. L. Hyman and R. Schuh ["Universals of tone rules, "Linguistic Inquiry 5, 81-115 (1974)] noted that tone spreading, in which a linguistic tone spreads its domain in time, is always perseverative, never anticipatory. I hypothesized that the mechanism processing $F_{0}$ might take more time than the mechanism processing segments, creating an auditory image in which $F_{0}$ would seem to "lag behind" segments. The results of a psychoacoustic experiment involving 21 subjects support this: an $\boldsymbol{F}_{\mathbf{0}}$ change and a formant change were judged to occur simultaneously most frequently when the tone change occurred $10 \mathrm{msec}$ before the center of the formant change. The apparent lag seems to cause listeners to perceive (and thus produce) tones as spreading into following segments.
The explanation should be approached cautiously: although the perceptual facts seem clear, it nevertheless remains to be seen whether a $10-\mathrm{msec}$ lag can lead to sound change. [Research supported by NSF.]

11:00

T13. Acoustic "before" and "after" of tonogenesis. E. Purcell, G. Villegas, and S. Young (Department of Linguistics, University of Southern California, Los A igeles, CA 90007)

Various investigators have examined the phonological details and implications of tonogenesis in a variety of languages. Fe'w have presented extensive phonetic dati on the development of tone in a language. We examined two related languages Panjabi and Hindi, the former having a three tone system, the latter not having a tone system. It has been hypothesized that the development of Panjabi tone is correlated with the simplication of an earlier system of consonantal oppositions, from a breathy voiced/voiced/roicele:ss system to a voiced/ voiceless system. Three native speakers of Panjabi and three native speakers of Hindi recorded samples of words which are cognates in the two languages. Ten measurements of fundamental frequency were made on each rowel of the test words. In all there were 4500 measurements of fundamental frequency. A comparison was made of average fundamental frequency for comparable word types in the Hindi and Panjabi data. The average data show that the Panjabi toral contours correlate well with Hindi fundamental frequency contours which vary according to the consonantal types adjoining vowels. In other words, Hindi consonantal types correlate with Panjabi tones.

\section{1: 10}

T14. Trill seeking. Sandra Ferrari Disner and Peter Ladefoged (Phoneties Laboratory, Linguistics Department, University of California, Los Angeles, CA 90024)

A comparative study was made of the rate and duration of trills produced with each of the three nonfixed articulators: the lips, the tongue tip, and the uvula. Recordings were made of initial, medial, and final trills produced by speakers of the New Guinea languages Kele and Titan 'bilabial trills); of Northeastern Italian, Ecuadorean and Columbian Spanish, Hausa, Kele, and Titan (apical trills); and of Southern Swedish, Northwestern Italian, and Southern French 'uvular trills). Instrumental analysis of these recordings revealed that the mean rate of trill ranges from 26 to 32 closures per second, sustained over a mean of $2-3$ closure.s. There is a great similarity between all these trills in the rate of vibration regardless of the mass of the articulator. Their auditory similarity will be demonstrated by means of a tape recording. [Research supported by NSF.]

\section{$11: 20$}

T15. Korean apical stop production: a VOT analysis. Barbara J. Moslin and John S. Knapp (Department of Linguisties, Box E, Brown University, Providence, RI 02912)

An investigation was made of the role of voice onset time (VOT) in distinguishing among Korean apical stop consonants. Measurements were made of VOT values for word-initial apical stops in the speech of four native Korean speakers. Words in citation form, in test sentences, in conversations among Korean adults, and in mothers' speech to children were used. Our results are not in accord with other experimenters who found complete separation of the aspirated from the strong and weak categories of Korean stops. VOT values for wordinitial apical stops in the speech of all four speakers showed considerable overlap of the weak and aspirated categories. The strong and weak categories were also found to overlap, but the overlap in the weak and aspirated cate zories was greater. Our data suggest that although VOT is sufficient to distinguish the strong from the aspirated stops, it carnot effectively distinguish either of these from the weak stops. Our findings support Kim (1965), who proposes a featu re of "tensity," independent of VOT, to distinguish weak stops from the strong and aspirated stops of Korean. 
11:30

T16. Rate estimates of sound-silence sequences in speech. Stanley Feldstein (Department of Psychology, University of Maryland Baltimore County, Baltimore, MD 21228)

Speech rate, a variable used frequently in psychological research, is usually indexed by number of words (spoken) per minute (WPM), the estimation of which is exceedingly time consuming. However, an automated temporal analysis of speech yields not words but a sequence of sounds and siliences. This study searched for a sequence paramoter to index rate as well as does WPM. Eighteen women were each asked to tell a different 3-min story in each of two conditions: in one she was to talk rapidly, in the other, to talk slowly. The automated analysis yielded four measures: average duratuions of vocalizations and pauses $(V, P)$ and proportionality constants of vocalizations and pauses $\left(\mathrm{PC}_{y}, \mathrm{PC}_{\emptyset}\right)$. The $\mathrm{PC}$ 's index the probabilities of continuing to vocalize or to remain silent within each successive time period sampled. Also, $\mathrm{PC}_{y} / \mathrm{PC}_{\phi}$ was calculated and WPM were obtained. After within-subject variance was accounted for, the sequence-derived parameter most highly related to WPM was $\mathrm{PC}_{v} / \mathrm{PC}_{p}(r=0.93)$. Moreover, $\mathrm{PC}_{v} / \mathrm{PC}_{p}$ distinguished between the conditions about as well as WPM. Relations between the sequence parameters and perceived sequence rates are now being examined.

\section{1:40}

T17. Voice fundamental frequency in saturation diving. H. Hollien and J. W. Hicks, Jr. (Institute for Advanced Study of the Communication Processes, University of Florida, Gainesville, FL 32601) and W. Shearer (Department of Speech and Hearing, Northern Illinois University, Dekalb, IL 60115)

Divers under hyperbaric conditions experience a marked deterioration in speech intelligibility. Included among the possible features that contribute to speech degradation is speaking fundamental frequency (SFF). Based on the physics of the environment and the physiology of the diver, it would not be expected that SFF would vary due to $\mathrm{HeO}_{2} / \mathrm{P}$ conditions. However, in an earlier pilot study, increase in SFF was found. In order to test the cited hypolhesis as well as expand the previous limited findings, a large group of U.S. Navy divers were studied. The diver/subjects produced speech at the surface and at depths of 200,450 , and $600 \mathrm{ft}$ in helium-rich environments. Of the original pool of 46 divers, 28 were studied at $200 \mathrm{ft}, 22$ at 450 and 9 at $600 \mathrm{ft}$. The speech material used was the first paragraph of the Rainbow Passage; SFF was obtained by processing this passage by FFI-6. The raw data revealed a significant rise in fundamental frequency to $450 \mathrm{ft}$ and a subsequent decrease at $600 \mathrm{ft}$, but subsequent analysis (based on the divers' SFF at the surface) showed an almost linear increase in SFF with increases in helium concentrate and ambient pressure. These results are discussed; it is believed that divers' attempts to speak "more intelligibly" (rather than the $\mathrm{HeO}_{2}$ environment) were the cause of this shift. [Work supported by ONR. ]

\section{$11 \cdot 50$}

T18. ChoraI speech and phonetic invariance. J. R. Cohen and J. J. Kupin (Department of Linguistics, University of Connecticut, Storrs, CT 06268)

In rhythmic choral speech, all speakers produce the same phonetic message at the same rate. A recording of such speech can be viewed as an algebraic summation in which speaker dependent variability is essentially reduced to the level of background noise. Intelligibility tests and acoustic analyses will be done on recordings of a large group of speakers producing short phrases containing stressed vowels in CVC syllables. The results will be considered in light of two competing theories of perceptual normalization; Kuhn [J. Acoust. Soc. Am. $58,428-433$ (1975)] which suggests that front cavity information is invariant across speakers; and the standard formant ratio hypothesis. Preliminary data from a mixed chorus of $\mathbf{3 0}$ members indicates that intelligibility is maintained for phrase length utterances, and further that several consonants and most vowels are readily perceptible, even in excised syllables.

WEDNESDAY, 17 NOVEMBER 1976

CABINET/FORUM ROOM,9:30 A.M.

\title{
Session U. Engineering Acoustics II: Polymer Transducers
}

\author{
James E. West, Chairperson \\ Bell Laboratories, Murray Hill, New Jersey 07974
}

Invited Papers

9:30

U1. Review of polymer transducers. G. M. Sessler (Technische Hockschule, Darmstadt, W. Germany)

Abstract not available.

$10: 00$

U2. Application of piezoelectric polymers to audio transducers. Takeo Yamamoto and Masahiko Tamura (Acoustical Engineering Research Laboratory, Pioneer Electronic Corp. , 4-2610 Hanazono, Tokorozawa 359, Japan)

Recently, piezoelectricity and pyroelectricity of polymer materials have attracted attention to the possibility of their application as new transducer materiaIs. The first study of the application of piezoelectric biological polymer materials, such as whale bones and tendons, was done by Fukada in phonograph cartridges. Later, synthetic polymer films, poly $(\gamma$-methyl $L$-gultamate), were used as transducing elements of experimental micro- 
phones and headphones; however, these transducers were not commercialized, because of their low sensitivity. In 1969, Kawai had found large piezoelectric effects in stretched and polarized poly (vinylidene fluoride) films. The stretched poly(vinylidene fluoride) films exhibit hysteresis characteristics similar to those of ferroelectric crystals under high alternating electric fields of $50 \mathrm{~Hz}$. After being subjected to a polarizing static field of about $600 \mathrm{kV} / \mathrm{cm}$ at $100^{\circ} \mathrm{C}$ for $40-60 \mathrm{~min}$, the film possesses large piezoelectric and py roelectric constants; for example, in the case of the transverse effect, the $d$ constant of the film is more than ten times larger than that of quartz, namely, $8 \times 10^{-7} \mathrm{cgs}$ esu. Reversible changes in infrared spectra before and after the poling process imply that the dipoles in the $\beta$ crystals of poly (vinylidene fluoride) are aligned along the direction of the applied electric field. The alignment is strongly affected by the properties of the amorphous region in the film. The coersive field in the $P-E$ hysteresis curve increases rapidly near the glass transition temperature. Not all of the dipoles in the $\beta$ crystals, however, can orient themselves along the poling field. Furthermore, this orientation is influenced by the crystal size and the degree of the crystallinity. This soft, thin piezoelectric polymer film has been applied to the design of various transducers, such as stereophonic headphones, direct radiator high frequency loudspeakers, microphones, phonograph cartridges, and very small accelerometers. These transducers have very simple structures because the diaphragms are piezoelectric filrns and possess the transducing function. Particularly, in the case of the loudspeakers, a pulsating cylinder may be easily obtained which has omnidirectional patterns up to $20 \mathrm{kHz}$. Some of these transducers have been commercialized already.

\section{0: 30}

\section{U3. Flexible electret transducers. G. K. Miller (GTE Sylvania, Mountain View, CA 94042)}

The availability of polymer electrets that can take on a wide variety of configuration: has made possible many new applications for electrostatic transducers. Electret materials, poling techniques, and basic transduction schemes are reviewed. Several specific line transducers are then examined both analytically and experimentally. A coaxial cable electret transducer is shown to be useable underwater as a hydrophone, underground as a stram detector, and on a fence as a vibration detector. A long multilayered tape with an electret layer is shown to be a line transducer that can be used in an active ultrasonic perimeter intrusion detection system. [Supported in part by ONR and by RADC.]

\section{Contributed Papers}

\section{1:00}

U4. Theory of vibrations of coated, thermopiezoelectric laminae. M. Cengiz Dökmeci (Massachusetts Institute of Technology, Room 5-207, Cambridge, MA 02139)

In this paper, we aim to construct a theory of vibrations of coated laminae in which there is coupling between elastic and electric as well as thermal fields. The laminae coated completely with perfectly conducting electrodes on both faces may comprise an arbitrary number of bonded layers, each with a distinct but uniform thickness, density and electromechanical properties. Within the framework of 3-D thermopiezoelectricity, we first develop a generalized variational theorem [cf. M.C. Dökmeci, L. Al Nuovo Cimento 7, 449-454 (1973)]. Next, following Mindlin [R.D. Mindlin, Int. J. Solids Struct. 10, 625-637 (1974)] and using this theorem, we construct a system of 2-D approximate governing equations of the coated laminae for the case when the mechanical displacement, electric potential and thermal field vary linearly across the laminae thickness. The effects of elastic stiffnesses of, and the interactions between, layers of the laminae and its electrodes are all taken into account. Lastly, we present theorems of uniqueness in the governing equations of the theory. And we examine special cases of interest. [Work supported by the Scientific and Technical Research Councll of Turkey. ]

\section{1:15}

U5. Flexural disk peizoelectric polymer hydrophones. J. M. Powers and T. D. Sullivan (Naval Underwater Systems Center, New London, CT 06320)

Piezoelectric polymer (poled polyvinylidene fluoride), a Japanese development recentiy introduced into high fidelity technology, shows promise as a hydrophone material because of its flexibility, ruggedness, low density, and potentially low cost. A family of lightweight flexural disk hydrophones which use piezoelectric polymer are described. Measurements on a 3.8-cm-diam unit gave a sensitivity of $-199 \mathrm{~dB}$ re $1 \mathrm{~V} /$ $\mu \mathrm{Pa}$ with good stability over ranges of $2 \mathrm{~Hz}-1 \mathrm{kHz}, 0^{\circ}-22^{\circ} \mathrm{C}$, and $0-4 \mathrm{MPa}$ static pressure. This perticular unit uses two air-backed flexural disks with a stack of four pieces of $30-\mu \mathrm{m}$ polymer film glued to each disk, resulting in a combined capacitance of $1 \mathrm{nFd}$. This hydrophone has a mass of $24 \mathrm{~g}$ in air and is neutrally buoyant in water. A theoretical analysis which shows the variation of hydrophone periormace as a function of design parameters is presented, and the material properties of piezoelectric polymer and piezoelectric ceramic are compared. [Work supported by NAVSEA and NAVELEX.]

$$
\text { 11: } 30
$$

U6. Effect of radiation loading on the equivalent circuit for a piezoelectric polymer transducer. A. 1 . Hudimac (Scientific Research Associates, Inc., 12100 Devilwood Dr., Rockville, MD 20854)

The equivalent circuit for a transducer using distended piezoelectric polymer sheets has been shown to be similar to that using a thin spherical shell, except that the mechanical impedance is given by a summation, in parallel, of impedances associated with a normal-mode expansion. The modal impedances are determined by the shape of the sheet boundary, the magnitude of the distension, and the nature of the radiation interaction between modes. The case of a distended circular sheet, fastened at the outer edge, radiating into a circular tube with rigid walls has been analyzed in detail. This can be used to approximate the behavior of one transducer in a infinite hexagonal array. The behavior of distended rectangular sheets in an infinite rectangular array in an infinite baffle has also been studied. In both cases, the radiation field is given. [Work supported by ONR. 1 


\title{
Session V. Psychological and Physiological Acoustics IV: Temporal Effects and Pattern Perception
}

\author{
Barry Leshowitz, Chairperson \\ Department of Psychology, Arizona State University, Tempe, Arizona 85281
}

Contributed Papers

$1: 30$

V1. Temporal integration of tone glides. M. J. Collins* and J. K. Cullen, Jr. (Kresge Hearing Research Laboratory. Department of Otorhinolaryngology, Louisiana State University Medical Center, New Orleans, LA 70119)

Temporal integration of fixed-frequency signals is a wellestablished phenomenon. However. integration characteristics for speech-like sounds are not known. The present study investigates temporal integration, in a background of noise, for fixed-frequency signals and rising and falling frequency contours in the ranges $200-700 \mathrm{~Hz}$ and $1200-1700 \mathrm{~Hz}$. These ranges were chosen because of their correspondence to firstand second-formant regions for speech sounds. Tone glides changed frequency linearly during the burst durations of 5,10 , $35,50,90$, and 120 msec. Reference fixed-frequency signals were selected at the extremes and arithmetic centers of the glide ranges (i.e., 200, 450, 700, 1200, 1450, and $1700 \mathrm{~Hz}$ ). The results for fixed-frequency signals are in agreement with previous investigations. The integration rate of the falling higher-frequency tone glide is similar to that of the fixedfrequency signals. However, other tone glides indicate a slower rate of integration than for fixed-frequency signals. The results are interpreted regarding time and frequency limitations on the development of the critical band, and possible relevancy to speech perception. [Work supported in part by NIH. 1

*On leave from Dept. of Communication, Tennessee State University, Nashville, TN 37203.

\section{$1: 45$}

V2. Masking of tone glides II. Igor V. Nábēlek (Department of Audiology and Speech Pathology, College of Liberal Arts, University of Tennessee, Knoxville, TN 37916)

Previous results for downward glides [J. Acoust. Soc. Am. 58, S34 (1975), Hearing and Davis (CID, St. Louis, 1976)] have shown that their masked threshoIds were higher than of constant tones. Present measurements with a larger range of stimuli confirmed the previous results and added new ones. Both directions of glides (between 2000 and 1800, 1000, 500. and $250 \mathrm{~Hz}$, respectively) were used. The burst duration was between 0.5 and $5000 \mathrm{msec}$, the sound pressure level of the masking white noise was $70 \mathrm{~dB}$. Listeners had normal hearing. Below approximately 10-msec duration the upward glides had lower thresholds than constant tones, the downward glides had higher thresholds. In a range between approximately 10 and $400 \mathrm{msec}$ both glides had higher thresholds than constant tones. The thresholds of downward glides below 200-msec duration were consistantly higher than of the upward glides, above 200 msec they were similar. Between 500 and $2000 \mathrm{msec}$ the glide thresholds were lower than the thresholds for the highest frequency and higher than the thresholds for the lowest frequeney of the glide. Generally, constant tone thresholds leveled off between 500 and $1000 \mathrm{msec}$, and glide thresholds at around $5000 \mathrm{msec}$.

$$
\text { 2:00 }
$$

v3. Subjective loudness of short duration signals. Michael Pavel (Bell Laboratories, Holmdel, NJ 07733)
It is known that the loudness of short signals with rectangular envelopes is proportional to the product of their power and their duration (i. e., their energy). This is consistent with the properties of a leaky integrator followed by a peak detector. As a result, it has been proped that this combination be used as a basis for measurement of loudness. The present study was conducted to determine the effect of nonrectangular envelope shapes on loudness judgments. The test stimuli, whose overall bandwidth was within a single critical band, consisted of a pure tone (1000 $\mathrm{Hz}$ ) whose amplitude was modulated by varlous slowly varying functions. The tested envelopes included combinations of rectangles, decay ing exponentials and growing exponentials. The durations ranged from 25 to 2000 msec. The test stimuli were presented binaurally and were compared in loudness to rectangular standard tones of the same frequency and duration. The points of subjective equality were determined by an adaptive 2IFC method. As expected, the shape of the envelopes had little effect on the loudness of the very short stimuli $(25 \mathrm{msec}$ ). Results for longer duration signals will be compared to predictions using an exponential integrator and a model proposed by Zwislocki. [J. Acoust. Soc. Am. 46, 431-441 (1969)].

$$
: \quad 2: 15
$$

V4. Perception of duration: Effect of a second signal nearby in time. R. Pokorny, R. Elmasian, and R. Galambos (Departments of Psychology and Neurosciences, University of California at San Diego, La Jolla, CA 92093)

Pairs of noisebursts ( $75 \mathrm{~dB}$ SPL, $150 \mathrm{msec}$ apart) were presented to subjects who were instructed to judge the duration of one of them (the target) and ignore the other (the conditioner). The two noisebursts could be presented to the same ear (monaural) or to opposite ears (dichotic), and the conditioner could precede or follow the target (forward and backward paradigm, respectively). Subjects estimated target duration by changing the duration of a third noiseburst (also $75 \mathrm{~dB}$ SPL) presented $1500 \mathrm{msec}$ after the pair. Five conditioner $(5,10,20,40$, and $80 \mathrm{msec})$ and two target durations (20 and $25 \mathrm{msec}$ ) were used. In the forward monaural paradigm the average subject selected bursts of 17 and $26 \mathrm{~m} \mathrm{sec}$ as matching the $20 \mathrm{msec}$ target when the conditioner was set at 5 and 80 msec, respectively. The matches showed smaller and more variable shifts in the monaural backward, dichotic forward, and dichotic backward paradigms. These perceptual duration shifts resemble a variety of other auditory illusions, such as loudness enhancement and pitch shifts [R. Elmasian and R. Galambos, J. Acoust. Soc. Am. 58, 229-234 (1975); 58, S83 (1975)] and may be related to them by a common mechanism.

$$
\text { 2:30 }
$$

V5. Duration shift and loudness enhacement: Separate but similar phenomena. R. Elmasian, R. Pokorny, and R. Galambos (Departments of Psychology and Neuroseiences. University of California at San Diego, La Jolla CA 920\$dr.

In duration shift (see Pokorny et al., th issue) and in loudness enhancement [e.g., J. A coust Soc. Am. 58, 229234 (1975)] subjective perceptions of one signal (the target) move toward those of another signal (the conditioner) presented 
nearby in time. Because the loudness of short signals increases with their duration (loudness summation), however, a duration shift experiment might merely be a loudness enhancement experiment in which subjects, contrary to instructions, match target loudness instead of target duration. The present study addresses this issue. Both conditioner duration (10 or 40 msec) and conditioner intensity $(61,75$, or $89 \mathrm{~dB}$ SPL) were varied in the monaural forward paradigm while subjects matched $75 \mathrm{~dB}$ white-noise targets of 20- and 25-msec duration. When they matched target duration in these 12 different conditions, changes in conditioner duration produced the greater shifts and when they matched the same signals for target loudness, changes in conditioner intensity produced the greater shifts. Thus, while cluration shifts and loudness enhancement share many common characteristics, they are separate perceptual phenomena.

\section{$2: 45$}

V6. Backward pitch-recognition interference. D.W. Sparks (Psychoacoustics Laboratory, Speech and Hearing Sciences, University of Washington Seattle, WA 98195)

Recent controversy concerning backward pitch-recognition interference has led some investigators to propose that this phenomenon is probably sensory in its origins; others believe that a disruption in the processing of a preperceptual auditory trace is occurring. Still others argue that pitch recognition disruptions result from an interference to an image, held in short-term store, which may have undergone at least an initial categorical assignment. Several experiments have been performed in which brief tonal-target bursts centered around 820 $\mathrm{Hz}$ were preceded or followed by interference signals. These interference signals included tones and noise bursts having a variety of durations, intensities, and center frequencies. Interference signals were ipsilateral or contralateral to the ear receiving the tonal targets. Aggregate results from these experiments support the conclusion that these disruptions occur after at least a preliminary categorical assignment.

\section{$3: 00$}

V7. Temporal resolution of components of word-length tonal patterns. C.S. Watson, B. Espinoza-Varas, and W.J. Kelly (Central Institute for the Deaf and Washington University, St. Louis, MO-63110)

We have recently reported that frequency resolution, under conditions of high stimulus uncertainty, is much more accurate for late high-frequency components than for early, low'frequency components of word-length tonal sequences [Watson, Wroton, Kelly, and Benbassat, J. Acoust. Soc. Am. 57, 1175-1185 (1975)]. We have now replicated these experiments for the case of temporal resolution. Listeners were tested with high, medium, and minimal stimulus uncertainty until they performed at asymptotic levels of temporal resolution, defined by just-detectable proportional increments $(\Delta t / t)$ in the duration of single 40-msec components of 10-tone sequences. The duration of late, high-frequency components is also resolved more accurately than that of early, low-frequency elements, although the differences are considerably less than for frequency resolution. A symptotic just-detectable values of $\Delta t / t$ range from about 0.75 to 0.15 , from high to minimal levels of stimulus uncertainty, suggestive of a shift from detecting increments in tonal sequence duration to detecting increments in the duration of single test components. [Supported by Grant \#NS-03856 from NINCDS.]

\section{3:15}

V8. Selective attentional cueing in auditory pattern discrimination. C.S. Watson and M. F. Spiegel (Central Institute for the Deaf and Washington University, St. Louis, MO 63110)

We previously reported that listeners can learn to "hear out" single 40-msec components of 10-tone sequences, when they are trained under minimal-uncertainty conditions. Once the highly aceurate frequency discrimination suggestive of selective listening has been achieved, it could be maintained even when the test component was $40 \mathrm{~dB}$ below the level of the pattern [Watson and Spiegel, J. Acoust. Soc. Am. 58, S84 (1975)l. Only a single component was tested in each pattern (e.g. , the second component was always tested in the eighth patterm). Thus, each pattern could potentially serve as a cue to the position of its test component. Extensions of these experiments, with same-different and with single-stimulus (remembered-standard) methods, support the following conclusions: (1) Once selective listening has been achieved for a specific pattern, it is maintained even when the pattern is presented at random with others. (2) The process of recognizing a particular pattern and then sele stively listening to the appropriate component within that pattern is very rapid. Since listeners can selectively attend to even the earliest components in patterns presented in the single-st:mulus procedure, the mechanisms of identification at least operate simultaneously with, and may precede, those of dise cimination. [Supported by Grant \#GMO-1900 from NIGMS to ' $N$. U. and Grant \#NS-03856 from NINCDS to C.I.D. I

\section{$3: 30$}

V9. Sequential two-channel frequency difference limen. W. A. Ahroon* and R. E. Pastore (Psj'choacoustics Laboratory, Department of Psychology, State University of New York, Binghamton, NY 13901)

The ability of trained observers to .nonitor the sequential inputs to two earphone channels was explored. In the independent same-different frequency discrimination tasks, the two temporally discrete standard stimuli were followed by two temporally discrete comparison stimuli such that the stimulus off set in the first channel was contiguous with the stimulus onset in the second. As with our earlier experiments [Ahroon, Pastore, and Wolz, J. Acoust. Soc. Am. 56, S45 (1974)], one-monaural and two-dichotic (seleclive-attention and dividedattention) listening conditions, temporal separation of the standard and comparison stimuli facilitated performance in the trailing channel in selective attention tasks, but not in the divided attention tasks. However, performance under both dichotic conditions was significantly poorer than monaural performance, with backward interference more disruptive than forward interference. In addition, the data give no support for a model based on an analogue to a real-time computer-interrupt handling system in two-channel discrimination experiments. [Research supported by a grart from NINDS. I

*Current address: Parmly Hearing Institute, Loyola University of Chicago, 6525 North Sheridan Rd., Chieago, IL 60626.

\section{$3: 45$}

V10. Contralateral cueing effects on backward masking. J.S. Puleo and R. E. Pastore (State University of New York at Binghamton, Binghamton, NY 13901)

Two yes-no detection experiments were used to investigate the effects of a contralateral cue on the detection of a brief $20-\mathrm{msec}, 500-\mathrm{Hz}$ signal followed by a monotic masking noise. The masking stimulus, a $20 \mathrm{~dB}$ increment in an otherwise continuous (41.5 $\mathrm{dB} A$ ) broadband noise, was presented at various specified time delays ( $1 \leq \Delta t \leq 320 \mathrm{msac}$ ) following the observation interval. The signal and cue were narrow bandpass filtered noise bursts in one experiment, and sinusoids in the other. Both signal $(p=0.5)$ and cue $(p=0.1)$ were presented during the same observation interval under three different phase conditions: $0^{\circ}, 180^{\circ}$, and randon phase. Relative to the no cue condition, the amount of backward masking found in both experiments under all cue conditions was significantly reduced $(\sim 3-5 \mathrm{~dB})$ at short delays $(\Delta t<20 \mathrm{msec})$, and only slightly reduced at longer delays. Under all cue conditions, the reduction in the amount of simultaneous masking and the reduction in backward masking at long delays was approximately equivalent. [This research was supported by a grant from NINDS. $]$ 
$4: 00$

v11. Lateralization by frequency in dichotic tonal sequences as a function of interaural amplitude and time differences. Diana Deutsch (Center for Human Information Processing, University of California at San Diego, La Jolla, CA 92093)

When listeners are presented with a dichotic sequence of tones which alternate in pitch between 400 and $800 \mathrm{~Hz}$, such that when one ear receives $400 \mathrm{~Hz}$ the other receives $800 \mathrm{~Hz}$ (and vice versa) many hear a single high tone in one ear alternating with a single low tone in the other. This effect is based in part on a lateralization of each fused tonal percept towards the ear receiving the $800-\mathrm{Hz}$ signal. It was found that in four out of five subjects, lateralization towards the $800-\mathrm{Hz}$ signal occurred even when the $400-\mathrm{Hz}$ signal was substantially higher in amplitude, and clearly higher in apparent loudness. However, although this effect was prominent with sequences consisting of 20 dichotic tone pairs, it was weak with sequences consisting of two dichotic tone pairs. With long sequences, lateralization to the $800-\mathrm{Hz}$ signal was also prominent even when it lagged or led the $400-\mathrm{Hz}$ signal by several milliseconds.

$$
4: 15
$$

V12. Ear dominance in diehotic chords and frequency discrimination in the right and left ear. P.L. Divenyl, R. Efron, and E.W. Yund (Neurophy siology-Biophysics Laboratories, Veterans Administration Hospital, Martinez, CA 94553)

In a series of experiments frequency discrimination thresholds were determinined for pure tones presented either to the right or to the left ear of experienced listeners. In some conditions the stimulus was monaural, whereas in others a tone of different frequency was simultaneously present in the contralateral ear. The difference between the frequency of the target in the discrimination task and that of the contralateral tone was $4-6 \%$. Center frequencies of $1.2,1.7$, and $3.2 \mathrm{kHz}$ were investigated. Results indicate that there is a small but reliable discrepancy between just-noticeable frequency differences obtained for the right and for the left ear, both in the monaural and in the dichotic conditions. Furthermore, the direction and the degree of asymmetry with respect to the frequency resolving power of the two ears was found to be strongly correlated with the direction and the degree of ear dominance for the pitch of dichotic two-tone complexes [see Efron and Yund, J. Acoust. Soc. Am. 59, 889-906 (1976)l. In other words, for a given subject and in a given frequency region, the ear which was more efficient in the frequency discrimination task was al so the one which dominated the pitch percept of a dichotic tone pair in the same frequency region. Implications of the relationship between the two types of functional asymmetry of the auditory system are discussed in the paper.

\section{$4: 30$}

V13. Perception of dichotic chords by hemispherectomized subjects. R. Efron (Veterans Administration Hospital, Martinez, CA 94553), M. Dennis (Psychology Department, Hospital for Sick Children, Toronto, Ontario, Canadal, J. E. Bogen (Ross-Loos Medical Group, Los Angeles, CA), and E. W. Yund (Veterans Administration Hospital, Martinez, CA)

The phenomenon of ear dominance for the pitch of dichotically presented tones has been described in previous publications [Efron and Yund, Neuro-psychologia 12, 249-256 (1974); $13,137-150$ (1975); 13, 151-161 (1975); Brain and Language 3, 246-254 (1976); J. Acoust. Soc. Am. 59, 889-898 (1976)l. In these studies on more than 100 normal subjects, the pitch mixture of the perceived dichotic chord was strongly dominated by the frequency presented to the right ear in $10 \%$ and by the left ear frequency in another $10 \%$. The remaining subjects $(80 \%)$ had either a weak or a nonsignificant ear dominance. Subsequent studies on subjects with forebrain commissurotomy [J. Acoust. Soc. Am. 59, S53(A) (1976)] indicated that the pitch mixture of the dichotic chord was determined by a functional asymmetry in a subcortical pitch processor. The present study with three subjects hemispherectomized in infancy and one at age 13 reveal a strong ear dominance for the ear contralateral to the existing hemisphere in all four subjects. These results suggest that there is efferent control, from the cerebral cortex, on the hypothesized subcortical pitch processor.

\title{
Session W. Noise IV. Prediction and Reduction of Industrial Noise Sources
}

\author{
Norman J. Meyer, Chairperson \\ Wyle Laboratories, El Segundo, California 90245
}

Contributed Papers

$$
1: 30
$$

W1. Prediction of construction site noise. F.H. Brittain, J. C. Freytag, and H. C. Chang (Bechtel Corp., P. O. Box 3965, San Francisco, CA 94119)

Prediction of construction site noise has generally been made by adding the highest noise levels generated by each piece of construction equipment. Worst case noise levels so predicted will overestimate actual noise levels. A technique has been developed to statistically $\left(L_{n}\right)$ predict construction site noise from a list of construction equipment and an assumed duty cycle. The effect of finite source size is included. Measured construction site noise levels are compared with predicted noise levels.
$1: 45$

W2. Impact sound level contour analysis of industrial sites. W. E. Bradley, W.F. Strong, F.T. DiBlasi, and J. M. Hood (Stone \& Webster Engineering Corp, 245 Summer St., Boston, MA 02032)

The evaluation of industrial sites, such as for nuclear or fossil power stations, often requires detailed analysis of the existing sound levels and acoustic impact of the proposed facility at each site. Such a study was performed for five potential power station sites according to the guidelines set forth by a state licensing agency with extensive site study requirements. As required by the state, ambient sound level contours, plant construction, operation and maintenance con- 
tours, and sound level impact contours were prepared for each site for the 12 required sampling periods. A variety of land uses ranging from rural to industrial-urban were included in the study. This paper summarized the techniques involved in developing and analyzing the sound level contours and discusses the applicability of the contouring process to the various acoustic environments.

$$
2: 00
$$

W3. Environmental noise of a nuclear power plant and its substations. E. E. Dennison, R.E. Maier, J.W. McGaughey, and S.P. Ying (Gilbert/Commonwealth, Jackson, MI 49201)

In order to fulfill government requirements for construction of a nuclear power plant, an environmental noise impact asassessment has been conducted. Ambient noise was measured continuously for 24 hour periods at the plant and substation sites and in the vicinity. Week long surveys were performed in each of the four seasons. A statistical interpretation of construction noise accounts for variability of construction equipment usage, its location and range of sound pressure levels. Histograms of construction noise and measured ambient noise are added to obtain the statistical representation of the predicted construction ambient noise. Operational noise of the plant is similarly predicted. The predicted noise contours of both operation and construction noise are formulated on the topographic maps. Meteorologic and topographic effects on the noise data are discussed.

\section{2: 15}

W4. A true integrating noise monitor. Paul D. Schomer (US A rmy Construction Engineering Research Laboratory, Champaign, IL 61820)

The Construction Engineering Research Laboratory of the Army Corps of Engineers has been faced with many stringent noise monitoring requirements by both unique Army needs and by special measurements for the Federal EPA. Artillery, armor, demolition, small arms, and helicopter together form a set of sources which are difficult or impossible to measure using standard meters with their associated time constants. Moreover, the true definition of $L_{d n}$ is an integral. These monitors possess new detectors which employ digital integration in order to eliminate the detector time constant problem. Also, to monitor in accordance with the new recommended impulse criteria, the new monitors are capable of incorporating a low-amplitude cutoff and $C$ weighting. Extreme heat, cold, rain, and snow present no problem; mechanical equipment has been eliminated from the basic monitor and uninterruptable power supplies employed. By the use of a microprocessor, flexibility is enhanced and currently these units are being modified to keep track of time of day and to be self-calibrating.$$
2: 30
$$

W5. Measurement of the average noise level for impulsive noise sources. L.C. Sutherland, R. E. Burke, and M.C. Lee (Wyle Research, El Segundo, CA 90245)

A wide range of artificial impulsive-type signals was measured with an impulse sound level meter to determine the relationship between the known (energy) average signal level and the observed meter reading for various settings of the meter time constant and frequency weighting network. The artificial signal consisted of tone bursts superimposed on continuous background noise. The tone bursts lasted from 1 to $100 \mathrm{msec}$, were repeated in intervals from 2 to $2000 \mathrm{msec}$ and had an rms level $10-50 \mathrm{~dB}$ above that of the random background noise. The latter was either pink noise or was shaped to resemble typical community noise. For impulse repetition rates greater than $1 \mathrm{pulse} / \mathrm{sec}$, the impulse sound level meter reading on the average within $\pm 1.3 \mathrm{~dB}$ of the energy average signal level, provided the meter was set on $A$-weighting "slow," the upper or maximum meter deflection was used, and this meter reading fell on the lower parts of the meter scale. Errors for lower pulse rates are also disucssed in this paper. [This work was supported by the U.S. Environment Protection Agency.]

\section{$2: 45$}

W6. Auger miner noise control. T.J. Retka (Donaldson Company, Inc., Minneapolis, MN 55440) and J. Alton Burks (U.S. Bureau of Mines, Bruceton, PA 15213)

Several auger miner machines were surveyed in underground coal mines to define operational noise levels at worker locations. Noise levels as high as $106 \mathrm{~dB} .4$ were recorded at worker positions with most noise levels exceeding $100 \mathrm{~dB} A$. Efforts were initiated to devise corrective measures to lower the noise exposure of operating personnel to $90 \mathrm{~dB} A$ or less. To perform a noise source identification study, an auger miner system was configured at a nonreverberatory aboveground test site which included provisions to operate in a coal-cutting mode. A comprehensive array of noise source diagnostic tests including (1) cover and expose noise testing, (2) vibration surveys, (3) impulse response (compliance) testing, (4) slowmotion movies, and (5) coherence testing were used to identify auger miner noise-producing components and mechanisms. The specific noise contributions of individual auger miner machine ry components were identified as a result of the source testing. During non-coal-cutting operation of the miner, frame radiation due to conveyor motion was found to be the most significant noise source. During coal-culting operation, radiation of augers, frame, and mine face were each found to be significant noise sources depending on worker locations. Noise reducing modifications for the auger miner have been devised, designed, and are being implemented. [Work supported by U.S. Bureau of Mines.]

\section{$3: 00$}

W7. Vibration damper for circular saws. C.H. Allen (The Clayton $\mathbf{H}$. Allen Coporation, 651 Concord Ave., Cambridge, MA 02138)

A new type of vibration damper for circular saws has been demonstrated by a preliminary working model that is applicable to conventional blades without decreasing the depth of cut. Early data show that the ringing, which usually lasts several seconds after an impact, dropped off in a fraction of a second, and the noise peak was reduced more than $10 \mathrm{~dB}$; as measured with the "fast" response of a sound level meter. The first operation of the damper while cutting a pine board (using a temporary arrangement) gave a $5-\mathrm{dB}$ reduction. When a proper installation is made, the noi se for the sawing operation is expected to be reduced as mucia as that for an impact. This paper will discuss the design and application of this new development and will present data from experiments now in progress.

\section{$3: 15$}

W8. Computer predictions of refinery noise levels. S. H. Judd and G. M. Walker (Standard Oil Company of California, 225 Bush St., San Francisco, CA 94104)

The predictions of a computer model [S. H. Judd, S. L. Dryden, and L. Tornheim, "Development of a community noise prediction model" and S. H. Judd and S. L. Dryden, "Application of a computer model for predicting remote noise levels", J. A coust. Soc. Am. 58, S16(A) (1975)] agree closely with the measured noise levels arouncl a major refinery. This is illustrated by octave band and $A$-weighted results. The construction of major new facilities which partially shield the existing plants has provided attenuaticn consistent with the algorithm used in the model as shown by before and after data. The model is being used to verify design predictions of source sound power levels and to assist in the selection of noise control measures.

\section{$3: 30$}

W9. Programming noise control in a small caliber munitions manufacturing plant. F.Z. Sachs and R.W. Heymann (U.S. Army Environmental Hygiene Agency, Aberdeen Proving Ground, MD 21010)

Program costs for the implementation of plant-wide noise control through retrofit of existing eqtipment were developed 
for a high-volume munitions manufacturing facility. Noise reduction costs were estimated by defining workable approaches for the control of all noise sources. Involved were over 1200 machines and some 37 different manufacturing operations. The principal noise sources were punch presses, parts hoppers, parts feed chutes, conveyor lines, tumblers, and other automatic metal-forming and transfer machines. The most prevalent generating mechanisms were the part impacts during transfer and part impact and surface vibration at the punch presses. The goal was to reduce the existing conditions, reaching $110 \mathrm{~dB} A$ in certa in locations, to below the U.S. Army's $85 \mathrm{~dB} A$ non-time-weighted hearing conservation criterion. The level of design detail of the noise control developed was limited to that required for cost estimation. Design detail for fabrication was not provided since the principal objective was to provide management with program cost data. This crucial information can now be factored into the capital equipment planning function along with traditional projections such as economic equipment life, future production requirements, and equipment modernization schedules.

$$
3: 45
$$

W10. Noise control for fan and vent shafts in subways. Peter Y.N. Lee (Wilson, Ihrig \& Associates, Inc., 5605 Ocean View Drive, Oakland, CA 94618)

Subway fan and vent shafts can be significant sources of noise impact to both the adjacent community and to patrons in the subway stations. For fan shafts, the transmission of airborne noise through the shafts and surface openings to adjacent buildings and the transmission of noise through the subways to station platform are intrusive. For vent shafts airborne noise transmissed from trains passing by in the subway below to surface openings ean be intrusive. The primary methods available for the reduction of fan and train noise propagated out shaft openings and fan noise propagated into the stations are (1) the use of acoustical absorption material on the interior surfaces of fan rooms, fan and vent shafts and tunnel walls and ceilings, (2) the use of sound attenuators [silencers] attached to the fans, (3) the use of specially constructed splitters in the fan and vent shafts or fan rooms, and (4) the use of acoustical louvers.

\section{4:00}

W11. Prediction of sound pressure levels in irregularly shaped factory spaces. J.H. Zinskie, L. D. Mitchell, and C.J. Hurst (Virginia Polytechnic Institute and State University, Department of Mechanical Engineering, Blacksburg, VA 24061)

The prediction of sound pressure levels in irregularly shaped factory spaces is approached through an analysis of acoustically coupled rooms. Previous work has indicated that room surface sound absorption characteristics create a theoretical interdependence between the sound pressure fields in each coupled volume. Whenever a barrier is interposed between a sound source and observer, the effects of diffraction must also be considered. A computer algorithm was developed to predict sound pressure levels in irregularly shaped rooms using a modified statistical acoustic theory. The program is based on a conversational technique and requires the user to enter information regarding room geometry, surface absorptions, receiver placements, and source sound power and placement information. Several output options are provided, among which is a plan view print-plot of sound pressure level contours in the analysis room. [Work supported by NSF.]

WEDNESDAY, 17 NOVEMBER 1976

SENATE/COMMITTEE ROOM, 2:00 P.M.

\title{
Session X. Physical Acoustics IV
}

\author{
Wayne M. Wright, Chairperson \\ Department of Physics, Kalamazoo College, Kalamazoo, Michigan 49007
}

\section{Contributed Papers}

\section{$2: 00$}

X1. Generalized theory of the photoacoustic effect. F.A. MeDonald and G.C. Wetsel, Jr. (Department of Physics, Southern Methodist University, Dallas, TX 75275)

The photoacoustic effect is the production of an acoustic signal when a sample in an enclosed cell is illuminated with chopped light. The absorbed light produces a periodic heat flow from the sample to the surrounding gas and backing material, causing pressure variations in the gas which are detected by a sensitive microphone. A theoretical treatment will be presented which involves simultaneous solution of thermaldiffusion equations for the sample and backing material and fluid-dynaraic equations for the gas. The resulting acoustic signal depends on the chopping frequency, the optical absorption coefficient and thermal properties of the sample, and on other material and system parameters. The conditions under which the approximate treatment of Rosencwaig and Gersho [J. Appl. Phys. 47, 64-69 (1976)] is valid will be discussed, Application of the theory to solid and liquid samples will be considered.

\section{$2: 15$}

X2. Experimental investigation of the photoacoustic effect in liquids and solids, G. C. Wetsel, Ir., and F.A. McDonald (Department of Physics, Southern Methodist University, Dallas, TX 75275)

The photoacoustic effect in liquids and solids has been experimentally investigated using light from helium-neon and argon-ion lasers. In an effort to evaluate the theor etical explanation of the effect (see preceding paper), the photoacoustic amplitude $Q$ has been measured as a function of chopping frequency $f$ for several liquids and solids with large values of the optical absorption coefficient $\beta$. The experimental results compare favorably with theor etical predictions over wide ranges of $f$ and $\beta$. Water solutions with varying concentrations of a common $\mathrm{pH}$ indicator were prepared to obtain a controlled evaluation of $Q(f, \beta)$. It was found that curve fitting the theory to the experimentally determined graph of $Q$ vs $f$ permits the determination of $\beta$ to at least two significant figures for a solute in water with a $\beta$ range of at least $50-5000 \mathrm{~cm}^{-1}$. This result indicates that the photoacoustic effect provides an 
important technique for determining the absolute optical absorption coefficient in highly absorbing liquids and solids.

$$
2: 30
$$

X3. Experimental meisurement of the modulation process involved in nonlinear interaction in water. C. R. Reeves, T. Go Goldberry, W. S. Olsen, and D. F. Rohde (Applied Research Laboratories, The University of Texas at Austin, P. O. Box 8029, Austin, TX 78712)

Nonlinear interaction between high and low frequency sound fields in water has long been modeled as phase modulation of the higher frequency by the lower frequency. For the modulation indices usually predicted, this implies the generation of side bands symmetric about the higher frequency having equal amplitudes but opposite phases. Simultaneous but independent measurements of the sidebands resulting from the interaction of a $65-\mathrm{kHz}$ signal with low frequency signals from $100 \mathrm{~Hz}$ to $1 \mathrm{kHz}$ now permit a direct comparison of the side bands for verification of the theory. Results are given for various frequencies, sound levels, and interaction geometries. [This research was supported by the Advanced Research Projects Agency of the Department of Defense and was monitored by the Naval Electronic Systems Command under Contract N00039$76-\mathrm{C}-0231.1$

$$
2: 45
$$

X4. First-order torques and spin velocities of solid bodies in intense sound fields. T. G. Wang and H. Kanber (Jet Propulsion Laboratory, Pasadena, CA 91103) and I. Rudnick (University of California, Los Angeles, CA 90024)

Time-average forces, torques, and velocities in sound fields have always been associated with second- or higher-order effects. We've identified, tor the first time so far as we know, phenomena which are first order in the acoustic particle velocity. In a rectangular box of square section the $(1,0,0)$ and $(0,1,0)$ modes are degenerate and when equally excited the particle motion is in general elliptical; it is circular if the phase difference of the modes is $\frac{1}{2} \pi$ and this is the case we'll have in mind. A long, small diameter, solid cylinder with its axis normal to the square cross section and at its center is then surrounded by a fluid whose particles are undergoing rotational motion (neglecting the scattering field of the cylinder). The cylinder experiences a torque due to viscosity and will rotate if free to do so. Moreover if the particle displacement is significantly greater than the viscous penetration depth the torque and velocity of rotation will be first order in the particle velocity. Measurements made on a one inch cylinder suspended by a wire, and in free rotation, in a $1.6 \mathrm{kHz}$ sound field, which support these conclusions, will be presented.

\section{$3: 00$}

X5. Acoustic diffraction by an impedance covered half plane. Robert P. Kendig (The Pennsylvania State University, Department of Engineering Seience and Mechanics, 227 Hammond Building, University Park, PA 16802)

Exact, closed form solutions for diffraction and backscattering by an edge whose surfaces exhibit arbitrary impedances were determined for the cases of a point source, a line source, and a plane wave. An integral method was devised that could account for the impedance boundary conditions. Since the solution is in a closed form, interpr etation of the influence of the impedance covered half plane on the diffraction field is greatly faciliated. Specifically, the effect of each surface on the acoustic field dominates in the half space in which it faces. Though the effect of each surface extends into the opposite half space behind the barrier, this influence is very small and diminishes even more as the observer moves further behind the barrier. [Work supported by NAVSEA.]

\section{$3: 15$}

X6. Wave interactions at plane and grating interfaces. M. A. Breazeale (Department of Physics, The University of

Tennessee, Knoxville, TN 37916)
Schlieren photography is used to show how an ultrasonic beam incident onto it liquid-solid interface couples in a resonant manner to a wave propagating laterally along the interface. At plane interfaces the lateral wave is a surface wave which produces a positive displacement of the reflected beam. At grating interfaces the lateral wave can result from a tangential diffraction order which can have a negative propagation vector. This leads to a negative displacement. The velocity of the lateral wave in this case is very nearly equal to the velocity of a compressional wave in the liquid. A motion picture shows the behavior of both types of interaction as the angle of incidence is changed. [Research supported by the L.S. Office of Navil Research. 1

\section{$3: 30$}

X7. Elastic wave scattering from ellipsoidal cavities, $B_{0} R$. Tittmann (Science Center, Rockwell International, P.O. Box 1085. Thousand Oaks, CA 91360)

Experimental results have been compared with theory for ellipsoidal and spherical cavities embedded in titanium alloy by the diffusion bonding process. The measurements comprised the cases of incident longitudinal and shear waves, including mode conversion. Whenever possible, comparisons were performed with the results of exact theory and those of the Born approximation. The Born approximation was found useful in the back seattering directions for low $k a$ values ( $k$ is the wave vector of the sound wave and $a$ is the radius of the scatterer). In the experiments, a reciprceity relation was discovered: The same angular dependence is obtained in mode conversion when the mode of the inciclent and scattered wave is interchanged. This result has now been corroborated by both the exact theory and the Born approximation. I'This work was partially supported by ARPA-AFML Contract No. F:3:3615$74-\mathrm{C}-5180$.

$$
3: 45
$$

$\mathrm{X}$. . Elastic properties of $\mathrm{Tb}_{0} \mathrm{Dy}_{0.7} \mathrm{Fe}_{2}$ measured ultrasonically. G. V. Blessing and A.E. Clark (Naval Surface Weapons Center, White Oak, Code WR-31, Silver Spring, MD 20910)

Ultrasonic velocity and absorption measurements have been made ats a function of magnetic field in both single crystal and polycrystalline $\mathrm{Tb}_{0.3} \mathrm{Dy}_{0_{0} 7} \mathrm{Fe}_{2}$. This highly magnetostrictive material exhibits a huge $\Delta E$ effect as previously reported at sonic frequencies. [A. E. Clark and H. T. Savage, IEEE Trans. Sonics Ultrason. SU-22, 50-52 (1975). $1 \Delta E$ is the difference in Young's modulus between the magretized and the unmagnetized state $E_{0 .}$. In a polycrystalline bar sample, we measured an increased extensional velocity as a function of applied field (to $6 \mathrm{KOe}$ ) which corr esponded to a $\Delta E / E_{0}$ of nearly $100 \%$. A peak in the low field relative attenuation, with a corresponding velocity minimum, has been observed. These measurements were made at room temperature over a frequency range of 0.05 to $0.25 \mathrm{MHz}$. Bulk shear and longitudinal velocity measurements have been made from 1 to $21 \mathrm{MHz}$ in both single crystal and polycrystalline samples. Measurements of sound propagation along $[110]$ show that the crystal is elastically isotropic. Taking the density to be $9.25 \mathrm{~g} / \mathrm{cm}^{2}$, at $12 \mathrm{MHz}$ we calculate $C_{11}=11.7, \frac{1}{2}\left(C_{11}-C_{12}\right)=3.84$, and $C_{44}=3.84\left(\times 10^{11}\right.$ $\mathrm{dyn} / \mathrm{cm}^{2}$ ). [Work supported by ONR.]

$$
4: 00
$$

X9. Microparticle detection with foctsed ultrasound, L. R. Abts, R. T. Beyer, P.D. Richardson, P. M. Galletti, and K. E. Karlson (Box D, Department of Engineering, Brown University, Providence, RI 02906)

A 5- $\mathrm{MHz}$ ultrasonic sensing device which detects and monitors microparticles in a moving fluid stream has been described, constructed, and calibrated. The ultrasonic transducer is so placed that a thin section of plexiglas exists between the transducer and the fluid stream. This section is of plano-concave shape and acts as a converging lens to focus the transducer at approximately $1.36 \mathrm{~cm}$ from the surface of the lens. The pulsed ultrasonic signal is transmitted into the fluid 
which flows through a calibrated and mapped sound radiation field. The calibration of the fleld for particle size was done through the use of carbonized microspheres, glass beads and air bubbles of known sizes. The device is calibrated to detect and monitor microparticles in the range of $7-400 \mu \mathrm{m}$ in diameter. A formula was derived from these calibration studies which relates reflected amplitude to particle size. The radiation field was mapped using a target ball of $0.635 \mathrm{~cm}$ in diameter. The apparatus has been used successfully during in vivo extracorporeal studies done on sheep, and has recorded microparticles during these procedures. [Work supported by Rhode Island Heart Association. I

X10. Reflection from absorbing cylinders. L. S, Schuetz and W. G. Neubauer (Naval Research Laboratory, Washington, DC 20375)

The theoretical solution for reflection of an acoustic plane wave by an elastic cylinder of infinite length, which may absorb energy, is formulated. The sensitivity of computations to the variation of physical parameters is discussed. Reflections as a function of frequency and angle, are compared with experimental data taken using finite cylinders immersed in water.

\section{$4: 30$}

X11. Effect of variations in properties of silicon rubbers on the scattering of sound, L. Flax and C.M. Davis (Naval Research Laboratory, Washington, DC 20375)

The scattering of plane waves by absorbing silicon rubber spheres and cylinders is investigated theor etically. The bodies are immersed in water. The effect of inclusion and exclusion of shear losses are examined. The scattering solution is calculated for a range of densities, velocities, and absorptions characteristic of many silicon rubbers.
$4: 45$

X12, Reflection of waves by a cylindrical cavity in an absorptive medium, L, Flax and W. G. Neubauer (Naval Research Laboratory, Washington, DC 20375)

Previous calculations of elastic wave reflections by cavities in solid materials have not included the effects of wave attenuation even for materials known to exhibit significant losses. Calculation without losses indicate the asymptotic approach of the scattering to a limiting value at some $k a$. The inclusion of losses modifies this behavior. Cases will be shown for reflections below $k a v 10$ and for materials with small and large losses,

\section{5:00}

X13. Monostatic cross section of a coated submerged hollow cylinder, G. C, Gaunaurd (Naval Surface Weapons Center, White Oak Laboratory, Silver Spring, MD 20910)

An elastic hollow cylinder in water is coated with a viscoelastic and sound-absorbing material layer, We previously found [Proc. III IIS/FRG Hydroacoustics Symposium, Munich, May 1975. Vol. 1, Pt. 2, 4-31, and J. Acoust. Soc. Am. 58, S101 (A) (1975)] the classical Rayleigh series for the pressure field in the water for the case of plane-wave insonification at beam aspect, from which a series expression for the backscattering cross section was derived. We now present the numerical evaluation of these results al relatively low frequencies $(k a \leq 20)$ in a lengthy and computerized parametric study. U'sing two size cylinders of interest, we analyze the effect of varying the coating viscosity, and the relative shell and coating thicknesses. The way in which the peaks in the oscillating cross section are shifted in frequency or dampedout in amplitude by changing geometries or materials, is useful to compute the coating effectiveness in reducing the target strength of the bare structure, a question of interest for design purposes. The high-frequency calculations remain to be performed using the Watson-Sommerfeld method.

\title{
Session Y. Underwater Acoustics IV: Scattering and Bottom Interactions. Precis Poster Session
}

\author{
Herman Medwin, Chairperson
}

Naval Postgraduate School, Monterey, California 93940

\section{Poster Session}

$2: 00$

Y1. Seasonal variations in volume scattering strength levels. W. B. Anderson (Naval Torpedo Station, Code 7023, Keyport, WA 98345)

Volume reverberation measurements have been conducted in the Straits of Georgia $(49.317 \mathrm{~N}, 124 \mathrm{~W})$ over a two-year period. Data were acquired with a downward-looking sonar system operating at discrete frequencies over a two-octave band $\left(f_{0}=35 \mathrm{kHz}\right)$. Supporting zooplankton samples were acquired with a fine-mesh plankton net trawled vertically through the water column under test. Acoustic data shows considerable variation in volume scattering strength $\left(S_{v}\right)$ levels to exist between seasons, generally supported by biological samples. In the fall/winter season a definite downward vertical migra- tion of acoustic scatterers is observed to begin at sunrise. A similar upward migration is observed at sunset. During the day the upper $100 \mathrm{~m}$ of the water column is essentially void of acoustic scatterers with a significant increase observed between 100 and $200 \mathrm{~m}$. The migrating scatterers begin from and return to this stratum. Review of calculated $S_{v}$ levels acquired during that seasonal time period indicate no variation of level as a function of frequency over the test spectrum. During the spring/summer season the upper $100 \mathrm{~m}$ show an increase in $S_{v}$ levels and caught zooplankton. The vertical migration, while still evident, is not the dominate phenomenon. Acoustic data shows the calculated $S_{v}$ levels to have a noted increase as a function of increasing frequency. This phenomenon appears restricted to the upper $200 \mathrm{~m}$. 
$2: 04$

Y2. High-frequency backscattering from mar ine zooplankton. Charles F. Greenlaw (School of Oceanography, Or egon State University, Corvallis, OR 97331)

It is aften assumed that backscattering from marine zooplankton may be satisfactorily estimated from the Rayleigh fluid-sphere scattering model at frequencies such that $k a<1$. At higher frequencies the fluid sphere model of Anderson [J. Acoust. Soc. Am. 22, 426-43(1950)] bas been thought approximately valid at least for the more spherical plankton. A series of acoustical and physical measurements was made on preserved specimens of three common zooplankters (a copepod, Calanus marshallae; an euphausiid, Euphausia pacifica; and a penaeid shrimp, Sergestes similis Hansen). Target strengths of individual specimens were measured in a freshwater tank at eight frequencies between 220 and $1100 \mathrm{kHz}$. Densities, compressional wave sound speeds, and displacement volumes of the samples were measured to enable calculation of predicted target strengths from the Ander son model. Measured target strengths of the copepod agreed with predictions up to $k a=1$, but were increasingly higher than predicted up to the highest $k a$ obtained $(k a \sim 4)$. The euphasusiid and sergestid were found to highly directional scatterers; anterior aspect target strengths were 10-20 dB lower than side or dorsal aspect values. Surprisingly, the general levels and gross structure of the side/ dorsal aspect target strengths were in reasonable agr eement with the Anderson model up to $k a \sim 11$ (euphausiid) and $k a \sim 7$ (sergestid). Data in the range $k a \leq 1$ could not be obtained for these plankters because of equipment problems. [Supported by ONR.]

\section{$2: 08$}

Y3. New model of resonant acoustic scattering by swimbladder-bearing fish. Richard $H$. Love (Naval Ocean Research and Development Activity, Bay St. Louis, MI 39520)

A new model of a swimbladder-bearing fish has been developed in order to provide improved predictions of the resonant frequency and acoustic cross section of such a fish. The model consists of a small spherical shell in water, enclosing an air cavity which supports a surface tension. The shell is a viscous, heat-conducting Newtonian fluid, with the physical properties of fish flesh. A comparison of the results obtained with the new model to experimental data indicates that the new model constitutes a definite improvement over previous models. The new model can predict the high values of damping and elevated resonant frequencies that previous models could not. The model appears to be most accurate for fish in which tension in the swim-bladder wall has a minor effect on resonant scattering. This includes the fish which are of interest in studies of volume reverberation and therefore, the new model should be of considerable value in such studies.

\section{$2: 12$}

Y4. Abstract withdrawn.

\section{$2: 16$}

Y5. Sound-scattering properties of curved surfaces with complex impedance. Jacob George and H. Überall (Department of Physics, Catholic University, Washington, DC 20064)

Specular reflection which accounts for most of the reflected signal at farfield depend on both the curvature of the scatter- er and its acoustic impedance. As specific eases, we calculate the backscattering cross section for plane waves reflected from circular cylinders and spheres. Results using both the Kirchhoff approximation and the Luneburg-Kline (LK) method (J. B. Keller et al., Commun. Pure Appl. Math 9, 207-265 (1956) $]$ are presented and compared. The LK expression for cross section contains uj to $(k a)^{-2}$ terms for the cylinder and up to $(k a)^{-1}$ terms for the sphere. The cross sections for the limiting cases of rigid and soft spheres are compared with those obtained by retaining up to $(k a)^{-1}$ terms in an asymptotic expansion. It is found that the LK method gives better results in the presence of a complex impedance. Dependence of the cross section on relative magnitudes of real and imaginary parts of the impedance are studied. Variation of the LK cross section with the angle of reflection is discussed. [Supported in part by ONR.]

$$
2: 20
$$

Y6. Relation between creeping waves and normal modes of vibration of a curved body. $H$. Utberall (Catholic University, Washington, DC 20064) and L. R. Dr chonette and L. Flax (Naval Research Laboratory, Washington, DC 20375)

The natural way for a disturbance to propagate over the surface of a smoothly curved, fluid-loaded elastic body is in the form of a series of damped circuinferential (creeping) waves. Mathematically, the process is most conveniently described by a sum of normal modes, each characterized by a wavelength that fits the body's circumference an integer number of times. We demonstrate that any such mode will resonate at all those "eigenfrequencies" where the mode velocity coincides with the speed of one of the creeping waves. For an elastic cylinder, the $180^{\circ}$ sound-scatter ing amplitude is shown to possess marked minima at many of the eigenfrequencies, whose spacing over a sequence of modes thus determines the group velocities of circumferential pulsed signals. [Work supported by ONR.]

$$
2: 24
$$

Y7. Amplitudes of transmitted and circumferential waves in sound scattering from an elastic cylinder. J. W. Dickey (Naval Ship Research Development Center, Annapolis, MD 21402) and H. Uberall (Catholic University, Washington, DC 20064)

The phenomenon of sound scattering from a smoothly curved, submerged elastic body involves two different mechanisms, namely (a) geometrical reflection, refraction and multiple internal reflections and (b) diffracted fluid-type and elastic-type surface waves. These have previously been studied individually [(a) Brill and Utberall, J. Acoust. Soc. Am. 50, 921 (1971); (b) Frisk, Dickey, and Überall, J. Acoust. Soc. Am. 58, 996 (1975), and (in press) ] for the case of an aluminum cylinder in water, obtaining dispersion and absorption curves for the diffracted field. We now evaluate the amplitudes of both (a) and (b) and compare their relative strength for values of $k a$ ( $k$ is the wave number and $a$ is the cylinder radius) from 25 to 200 . While the lower-order geometrical contributions are generally larger than the diffracted ones by an order of magnitude, some of the latter may surpass the former, and the relative elastic-type (Rayleigh etc.) surface-wave amplitudes do not markedly decrease with ba while the fluid-type waves (Franz, Stoneley) do. We have searched for resonances in the surface-wave amplitudes, possibly related to mode resonances at their eigenfrequencies.

$$
2: 28
$$

Y8. Theory of scattering by rigid bocies with thin liquid coatings, T. J. Eisler (Department of Mechanical Engineering, Catholic University, Washington, DC 20064)

We consider a plane-wave incident on a rigid body which is partially or totally surrounded by a thin liquid coating whose (possibly variable) density and sound velocity differ from those of the ambient medium. The wavelength is assumed to be small compared with the dimensions of the body but large 
compared with the thickness of the coating. The scatter ed wave is expressed in terms of integrals similar to the Kirchhoff integral. These are derived by iteration from an integral formulation. The first iteration produces corrections to the uncoated solution which are linear in $k \delta$, where $k$ is the wave number of the incident wave and $\delta$ the thickness of the coating. We restrict our attention to these linear terms. The scattering cross section may be evaluated in terms of contributions from the specular point and from points on the edge of the coating at which the incident rays are perpendicular to the edge. In the absence of absorption it is found that the edge contribut $10 \mathrm{n} \mathrm{s}$ Technology Program, ONR.l Dominate

\section{$2: 32$}

Y9. Measurement of the acoustic field scattered at the liquidsolid interface of an echelette grating. S. K. Numrich (Naval Research Laboratory, Code 8123 , Washington, DC 20375)

Experimental measurements have been made of the acoustic field produced by sound scattered at the brass-water interface of an echelette grating. Theoretical calculation of the reflection coefficients for the various orders of scattered waves have been completed by J. DeSanto. Schlieren photographs are used to indicate the angular dependence of the scattered field and hydrophone measurements of the acoustic field are compared with the theoretical model.

$$
2: 36
$$

Y10. Measurements of underwater forward scattering of acoustic signals by the sea surface, T.D. Plemons and E. A. Pence, Jr. (Applied Physics Laboratory, University of Washington, 1013 N. E. 40th Street, Seattle, WA 98185)

Underwater forward scattering measurements of acoustic signals were made this year in Dabob Bay located on Hood Canal in Washington State. Frequencies of 15, 20, 25, and $30 \mathrm{kHz}$ were transmitted in 1.0 -msec pulses. Signals reflected from the wind-driven surface of Dabob Bay were measured in the specular direction with grazing angles varying from $5^{\circ}$ to $23^{\circ}$. For each grazing angle, surface roughness and transmitted frequency, 100 reflected signals were processed to estimate the average and the coefficient of variation of the scattered energy. The experimental probability density and distribution functions of the scattered energies were also constructed. The dependence of each of these statistical quantities on the surface roughness parameter $g$ is discussed. For an rms surface-wave height $\sigma$, grazing angle $\theta$ and acoustic wavelength $\lambda, g$ $=4 \pi \sigma \sin \theta / \lambda$.

\section{$2: 40$}

Y11. Goniometric measurement of power scattered from wind driven water surfaces, J. G. Zornig (Department of Engineering and Applied Science, Yale University, New Haven, CT 06520)

A recently constructed 11-ft, 3-axis, semiautomatic underwater goniometer has made possible precision measurement of acoustic power scattered from the statistically stationary wind driven water surface at Yale. Measurements have been made over $180^{\circ}$ of azimuth and at several grazing angles. These measurements have been corrected for beam patterns and pulse shape to give a scattering cross section for the surfaces and geometries studied. The results of these measurements indicate that current mathematical models of the scattering process are not able to predict spatial distribution of scattered power in all cases.

$$
2: 44
$$

Y12. Scattering function for underwater acoustic scatter channels with several bounces. Franz B. Tuteur (Department of Engineering and Applied Science, Yale University, New Haven, C'T 06520)
The scattering function, or range-Doppler plot for an underwater acoustic channel involving a single surface bounce was derived in a recent paper (J.F. McDonald and F.B. Tuteur, J. Acoust. Soc. Am. 67, 1025-1029 $(19 \times x) 1$ and was shown to have a characteristic horse-shoe shape when the surface waves have a well-defined direction. This shape indicates strong correlation between Doppler shifts and range delays. In practice underwater acoustic channels often involve several reflections, some from the surface and some from the bottom. A calculation of the scattering function for such channels has been performed using an extension of the Kirchhoff integral method described in [F. B. Tuteur, J. Acoust. Soc. Am, 60 (October 1976) ]. For a channel involving a single bottom bounce and surface bounce the reverberation time and the Doppler spread are found to be weighted averages of the respective quantities for single bounces, with weighting factors that depend on the geometry and on the statistics of both surface and bottom. The shape of the scattering function can ther efore be expected to be qualitatively similar to the one for the single surface bounce.

Y13. Stochastic characterization of propagation and target impulse-response measurements. Will iam D, Mark (Marine Sciences Department, Bolt Beranek and Newman Inc., Cambridge, MA 02138)

In many situations, propagation and target impulse-response measurements are most conveniently treated as stochastic transients. Ideally, stochastic characterizations of such impulse-response measurements should possess input-response relationships which permit combination of characterizations of one-way transmission and target impulse-response functions with sonar signals and receiver impulse responses to yield predictions of ensemble-averaged instantaneous (time-varying) squared receiver responses. Two spectral methods [W. D. Mark, J. Sound Vib. 22, 249-295 (1972), J. Acoust. Soc. Am. $59,1184-1194$ (1976) ] of characterization of stochastic impulse-response measurements will be described, and properties of series representations of ensemble-averaged squared sonar receiver responses, generated from these characterizations, will be discussed.

X

Y14. Range dependence of underwater echo from random rough surfaces. E. E. Mikeska and C. M. McKinney (Applied Research Laboratories, The University of Texas at Austin, Austin, $\mathrm{TX} 78712$ )

The spreading-loss portion of the total propagation loss of sound reflected or scattered from a rough ocean bottom is not firmly established in the literature. Many investigators of bottom backscattering assume, for non-normal incidence, spherical spreading both from the source to the bottom and for the return path; this procedure yields a spreading-loss correction term of $-40 \log R$ (where $R$ is the one-way range in yards). At normal incidence, the mirror-image solution is generally assumed for bottoms which are not too rough relative to the wavelength, resulting in a spreading loss term of $-20 \log (2 R)$. To clar ify the actual range dependence of the returning echo, experimental measurements were made in a laboratory tank on the echo from randomly rough model surfaces having known statistical properties. Data are available for normal incidence at $100 \mathrm{kHz}$. The echo from a plane surface was checked and confirmed to result in mirror-image behavior, with a spreading loss of $6 \mathrm{~dB}$ over the return path. The echo from a random rough surface having surface irregularities comparable to a wavelength fluctuated greatly with range and with small changes in the particular area of surface insonified, but an average of many range dependence curves shows an echo-amplitude decay rate of $13.5 \mathrm{~dB}$ for a tenfold increase in range, beginning about $2 \mathrm{ft}$ from the reflecting surface. 
$2: 56$

Y15. Shear waves in unconsolidated sediments. D. J. Shirley and A. L. Anderson (Applied Research Laboratories, The University of Texas at Austin, P. O. Box 8029, Austin, TX 78712)

The dynamic shear modulus of unconsolidated marine sediments is small but nonzero. Shear waves will propagate in the sediments, but only at very slow speeds. The compressional wave speed is increased as much as $5 \%$ by the nonzero modulus. As a suspension of clay particles settles and collects into a gel-like material, the dynamic shear modulus increases. This initial development of sediment frame structure has been observed by measuring shear wave speed. Several stages in the development process can be described from these results.

$$
3: 00
$$

Y16. Influence of surface waves on plane wave bottom reflection loss for realistic ocean sediments. Kenneth E. Hawker (Applied Research Laboratories, The University of Texas at Austin, P. O. Box 8029, Austin, TX 78712)

Plane-wave reflection coefficients have been computed for several cases of fluid sediments, containing a constant soundspeed gradient, overlying a homogeneous solid substrate. It is shown that for realistic sediment descriptions, a pronounced maximum in bottom loss can occur at low grazing angles (well below the shear-wave critical angle). This maxima is well defined in angle and can occur only for special combinations of sound-speed gradient, frequency, and sediment thickness. It is shown that this phenomena is due to Stoneley waves propagating in the neighborhood of the sediment-substrate interface. These surface waves can become excited only when their phase velocity equals the parallel component of the phase velocity equals the parallel component of the phase velocity of the incident plane waves. Calculations are presented showing excellent agreement between the location of the bottom loss maxima and the predictions of a simplied theory based upon the excitation of Stoneley waves. The frequency, layer thickness, and sound speed gradient dependence are all adequately accounted for.

\section{$3: 04$}

Y17. Acoustic reflection from sea bottom with linearly increasing sound speed. A. O. Williams, Jr. (Applied Research Laboratories, The University of Texas at Austin, Austin, TX 78712 , and Brown University, Providence, RI 02912) and Douglas R. MacAyeal (Brown University, Providence, RI 02912)

We calculate the acoustic reflection coefficient for plane waves, in a homogeneous fluid half space, incident at arbitrary angle upon an underlying half space of bottom sediments. The latter, treated here as a fluid of constant density, has a sound speed $c$ increasing with depth $z, d c / d z$ being constant (a behavior frequently encounter ed). An exact formal expression is found for the complex reflection coefficient $R$, in terms of a modified Bessel function. With no absorption, $|R|$ is unity for all angles, as expected from ray theory. Assuming bottom absorption, we derive an analytic approximation for $R$, valid at low acoustic frequencies (up to a few kilohertz, at least). Numerical results are described. Incident angles too near the normal must be excluded, but a different approximation serves for small angles, including normal incidence. A more realistic model having layers in the upper part of the bottom is examined. In effect, it now reduces to problems already solved. [Supported by Naval Electronics Systems Command, Department of the Navy, Washington, DC, under Contract N00039-76-C-0081. 1
$3: 08$

Y18. Determination of the acoustic properties of deep-ocean sediments from in situ profiles. D. J. Shirley (Applied Research Laboratories, The University of Texas at Austin, Austin, TX 78712)

The acoustic properties of ocean sediments are routinely measured in situ during coring using the ARL sound-speed profilometer. Measurements are made at $200 \mathrm{kHz}$ across the diameter of the core during penetration of the corer into the ocean bottom. Profiles measured on the Southwest Bermuda Rise, the West Antilles Outer Ridge, and the Nares Abyssal Plain in water depths greater than $5 \mathrm{~km}$ indicates that some of the acoustic properties associated with sediments of these areas are: (1) a sound speed at the top of the sediment that is $20-30 \mathrm{~m} / \mathrm{sec}$ slower than that of the overlying water, (2) a linear increase in the gross sound speed of the sediment of from 0.5 to $1.0 \mathrm{~m} / \mathrm{sec}$ per $\mathrm{m}$ depth, and (3) a fine structure of high and low speed layers impressed on the gross strueture. [This work was supported by ONR, Code 480 .]

\section{$3: 12$}

Y19. Ocean sub-bottom refraction. A significant propagation mechanism at low frequencies. R. K. Dullea, P. D. Herstein, and S. R. Santaniello (Naval Underwater Systems Center, New London, CT 06320)

Experimental studies by independent investigators have indicated that low-frequency acoustic propagation can be affected by ocean-bottom refracted energy. However, the total energy returned from the bottom is also comosed of water-sediment interface and sub-bottom reflected energy and it is often times difficult to determine the significance of the sub-bottom refracted energy. Deconvolution processing techniques applied to wide-band explosive-source experimental data have aided in time-isolation of the various bottom-interacted arrivals so that signal travel times could be determined. With the use of a raytracing model which incorporated a sediment sound-speed gradient, accurate identification of sub-bottom refracted arrivals has been achieved. Spectral analyses of these data have further revealed that sub-bottom refracted energy can exceed reflected energy by as much as $5-10 \mathrm{~dB}$ at frequencies below $500 \mathrm{~Hz}$. This effect has been observed for diverse geographical areas of the North Atlantic (shallow basins, abyssal plains) where sediment structures vary considerably. As a consequence, acoustic properties of the ocean bottom cannot be correctly inferred at low frequencies by a Rayleigh reflectivity model. [Work supported by NAVSEA.)

\section{$3: 16$}

Y20. Abstract withdrawn. 


\title{
Session Z. Architectural Acoustics III: Open Planning, Masking, Reverberant Rooms
}

\author{
David Lubman, Chairperson \\ David Lubman and Associates, Woodland Hills, California 91367
}

\section{Contributed Papers}

2:00

Z1. Acoustical environment in the open-plan office. D. A. Harris (Owens-Corning Fiberglas Corp., Fiberglas Tower, Toledo, OH 43659)

ASTM E-33.04C Committee on Environmental Acoustics, Applications Subcomittee, Task Force on Open-Office Acoustics, has prepared a publication entitled, "Acoustical Environment in the Open-Plan Office." The purpose was "... to assemble and explain recent references on the acoustical design and testing of open-plan offices. The principles contained may be used by the Committee to prepare applicable ASTM standards and recommended practices for the acoustical evaluation of open-plan office systems." The paper deals with the "... acoustics of the open-plan office in a way that clarifies the principles that designers and architects use to achieve an acceptable and flexible environment." It covers privacy criteria and theory, how open office components affect the acoustical environment, a discussion of appropriate testing procedures, and a summary of acoustical design parameters. A summary of the paper as published in ASTM Standardization News will be presented.

$$
2: 15
$$

Z2. Guide for users and designers of open plan classrooms. Bill Siekman (Leisure Time Enterprises, Hixton, WI 54635)

In 1972 the Acoustical Insulating Materials Association sponsored a program of research by Tracor, Inc., to develop guidelines for optimum design and use of open-plan classrooms, particularly in regard to the effective use of acoustical absorption. This program utilized literature study, visits to open-plan schools, and computer simulation of large rooms to develop performance criteria and procedures for evaluation. One very important conclusion from this study was that less than $10 \%$ of existing classrooms were considered acoustically excellent, and two-thirds were just tolerable or worse. Fortunately, the features which distinguished the good rooms were conspicuous and definable. In 1975, the highly technical report from Tracor was rewritten in different language for use by teachers, school administrators, and architects. Engineering data has been reduced to simple tables, charts, and worksheets. Recommendations for accomodation to existing classrooms have also been incorporated. The Guide is now ready for publication and distribution.

$$
2: 30
$$

Z3. Variable and adaptive masking sound systems. Robert $\mathbf{C}$. Chanaud (Dyna-Systems, Inc., 2709 West 27th Ave., Denver, CO 80211 )

Increased usage of masking sound systems in open plan offices has shown that certain additional features are both useful and desirable and these will be described. One such feature is the automatic control of both level and spectrum throughout the day, including automatic, but slow, shutoff to conserve energy. Another feature is multiple use of the loudspeakers. Both paging and music have been superimposed on the masking sound alr eady. In addition it is possible to have the masking sound controller respond to alarm signals, such as a smoke detector, activate a prerecorded taped message, and give emergency instructions over the sound system. Other uses, such as nighttime security monitoring, will be described. A number of masking sound spectra have been used in open offices but we have found that a modified PNC-40 contour yields minimum Articulation Index for a given $A$-weighted sound level.

$$
2: 45
$$

Z4. Vibration induced masking noise. Howard N. McGregor (Engineering Dynamics, Inc., $6651 \mathrm{~s}$. Wellington Ct. , Littleton, $\operatorname{CO} 80121$ )

Very high acoustical gradients negate acoustical privacy in buildings which normally have acceptable transmission loss between units. This is particularly true of mountain condominium complexes which have entertainment areas in the same building. The vibro-mask system reduces the acoustic gradient by introducing masking noise into the quieter areas such as sleeping quarters. Acoustical transducers induce structural vibration which is in turn transmitted throughout the building with a reasonable degree of uniformity. Equalization of the input power spectrum is accomplished by electronic networks to obtain the desired masking sound spectrum.

\section{$3: 15$}

Z5. Experimental investigation of the effect of a rotating diffuser upon the radiation impedance of a source in a reverberant room. C.H. Hansen and D. A. Bies (Department of Mechanical Engineering, University of Adelaide, Adelaide, South Australia 5001)

The radiation impedance of an aperture in the side wall of a $180 \mathrm{~m}^{3}$ reverberant room has been measured over the frequency range from 100 to $2000 \mathrm{~Hz}$ using a simple impedance tube and a high speed data acquisition system which allowed subsequent data reduction on a CDC6400 computer. The room contained a rotating diffuser of $18.71 \mathrm{~m}^{3}$ of swept volume which was capable of continuously variable rotational speed up to $50 \mathrm{rpm}$. Two apertures were investigated which were the open end of a 10-cm diameter impedance tube and the 43-cm diameter mouth of a conical horn. In the case of the horn the throat impedance was measured. Measurements at approximately two degree intervals of diffuser rotation showed that on average free-field values of radiation impedance obtained at all frequencies tested and for all rotational speeds of the diffuser. However a marked increase in the standard deviation about the mean was observed at frequencies below $900 \mathrm{~Hz}$ which decreased with increasing diffuser rotational speed up to approximately $30 \mathrm{rpm}$ after which no further decrease occurred.

$$
3: 30
$$

26. Experimental comparison between Mariner's approximation and an exact solution of equations describing the measurement of transmission loss of a test partition. D. A. Bies and J.M. Pickles (Department of Mechanical Engineering, University of Adelaide, Adelaide, South Australia 5001)

The American standard for the laboratory measurement of the transmission loss of a test partition, E90-75, recommends the use of a procedure developed by Thomas Mariner [J. Acoust. Soc. Am. 33, 1131-1139 (1961)] which seeks to minimize the effect of coupling between the source and receiving rooms during calibration of the receiving room by the rever- 
berant decay method. When Mariner's reverberation decay "tuning" is carried out his approximate solution may be used and the possible error in the measurement may be estimated using a procedure which he has outlined. In this paper a comparison is made between Mariner's approximation and an exact solution of the problem as originally outlined by Mariner but not considered by him. The comparison will include a brief outline of the solution as well as experimental work comparing the two methods. The exact solution does not require reverberation tuning but it does require measurements of reverberation in both rooms as well as noise reduction measurements in both directions.

\section{$3: 45$}

Z7. Effect of source position on sound power radiation in a reverberant chamber. Gary Mange (Wyle Laboratories, 128 Maryland St. , El Segundo, CA 90245) and Dr. Jiri Tichy (Applied Research Laboratory, P.O. Box 30, State College, PA 16801)

A model reverberant chamber was utilized to measured the variance of radiated sound power of a one inch dome loudspeaker. The chamber was excited by five uncorrelated pure tones at four source positions and the reverberant sound field was spatially sampled with two moving microphones. The spatial variances and the variance of the radiated sound power of the source were determined in five octave bands. The above procedure was repeated for four chamber conditionswith and without added absorption, and with and without a rotating diffuser operating. The experimental values of the variance of radiated sound power showed excellent agreement with previously published results [G. C. Maling, IBM J. Res. Dev. 11, 492-501 (1967)l and were also systematically lower than the theoretically predicted values based on a "next neighbor" Poisson distribution of modes. The added chamber absorption reduces the variance at low frequencies as predicted by theory, but the rotating diffuser had very little effect on this variance.

\section{$4: 00$}

Z8. Measurement of reverberation time in the National Bureau of Standards reverberation room. Thomas W. Bartel and Edward B. Magrab (Institute for Basic Standards, National Bureau of Standards, Washington, D.C. 20234)

The spatial variation of the reverberation time in the $1 / 3$ octave bands from 80 to $10000 \mathrm{~Hz}$ was studied as a function of: (1) the surface area of the absorbing material ranging from 0 to $13.4 \mathrm{~m}^{2}$; (2) the orientation and rotational speed (0-10 rpm) of the vanes; (3) loudspeaker placement and type; (4) discrete microphone versus high-speed multiplexed data acquisition; (5) the number of microphones; and (6) the number of decay times averaged at a given microphone location. For the case of the vanes rotating at $7.5 \mathrm{rpm}$ it was found that the standard deviation of spatial variation of the reverberation time from 12 microphones ranged from $1 \%$ to $12 \%$ of the mean value in the frequency bands below $400 \mathrm{~Hz}$, was less than $0.5 \%$ from 400 to $5000 \mathrm{~Hz}$ and increased to $1.5 \%$ at $10000 \mathrm{~Hz}$. The values were virtually independent of the surface area of the absorbing material placed in the room. With the vanes stationary these percentages increased by as much as a factor of 5 . In addition to the decrease in the spatial variance with the vanes rotating, the spatially-averaged reverberation time decreased by $2 \%$ to $15 \%$ in the frequency bands below $500 \mathrm{~Hz}$.

$$
4: 15
$$

Z9. Automatic measurement of reverberation times using a real-time analyzer and a computer. Roger Upton (Brüel \& Kjær, Linde Allé 23, 2850 Nærum, Danmark)

Attempts to measure reverberation times automatically using real-time analyzers with computers have failed in the past due to the available analyzers having averaging times that are too long or insufficient dynamic range. With the advent of the Brüel \& Kjar Digital Frequency Analyzer type 2131, however, this is no longer the case. A method of using this instrument with a computer or a desk top calculator is described, whereby reverberation times can be measured automatically in parallel in all octaves or $\frac{1}{3}$ octaves of interest, within the frequency range of the analyzer. Time clomain resolutions of up to approximately 22 points per second can be obtained, and the method can be used either with interrupted noise or impulsive noise as the excitation. Comparison is made between experi-mental results obtained using the method, and those obtained using traditional techniques.

$$
4: 30
$$

Z10. System for the measurement of acoustic energy density. Bruce L. Morton and Elmer L. Hixsion (Department of Electrical Engineering. The University o: Texas at Austin, Austin, TX 78712)

In the reverberation chamber method of measuring the sound power radiated from an acoust.c noise source, pressure microphones are used in arrays, trayersed over fixed paths or used singly with a rotating diffuser. The purpose is to accurately measure the potential energ. density in the sound field produced by the noise source. In general, accuracy is limited by the variance in the measurement of the potential energy density. When the total energy density (potential and kinetic) is measured, the variance should be cut in half. A system has been designed and built that measure; the total energy density in a three-dimensional sound field. Three sets of spaced pressure microphones are placed orthogonally. For a set the signals are averaged and squared to produce the potential energy at a point and the difference signal is squared to give the square of one component of the acsoustic velocity vector. By multiplexing the sum of the squares of the three components of the velocity vector the kinetic energy is produced. The three pressure squared signals are averaged to produce the potential energy, halved and added to half the kinetic energy to give total energy density. Calibration is accomplished with an acoustic calibrator or pistonphone and small plane wave tube. Measurements made in one dimensional and three dimensional sound fields will be presented.

\section{$4: 45$}

Z11. Equivalence of eigenmode and free-wave models of steady-state reverber ation in rectangular rooms. David Lubman (D. Lubman \& Associates, 2372' Crosson Drive, Woodland Hills, CA 91364)

The eigenmode and free-wave models of steady-state reverberant sound field in rooms are sometimes mistakenly believed to be unrelated representations. These two models are shown to coincide for the case of rectangular rooms, for which the axial, tangential, and oblique modes can be decomposed into two, four and eight travelling plane waves, respectively. (This was pointed out by Morse and Bolt [Rev. Mod. Phys. 16 (2), 85 (1944)].) Above the frequency of modal overlap, therefore, the reverberant sound field in a rectangular room may be viewed as an ensemble of many plane waves arriving from many directions in space. This provides justification for use of the free-wave model.

\section{5:00}

Z12. Spatial standard deviation of SPL in National Research Council's transmission loss laboratory. W. T. Chu (Division of Building Research, National Research Council, Ottawa, Ontario, Canada) and David Lubman (D. Lubman \& Associates, 23727 Crosson Drive, Woodland Hills, CA 91364)

The accuracy of transmission loss (TL) determinations depends in part upon the precision of measured noise reduction (NR). This is controlled by the spatial standard deviation of sound pressure level $(\sigma, \mathrm{dB})$ in both siource and receiving rooms. National Research Council is obtaining experimental results to test a theory proposed by Iubman and Bowers [J. Acoust. Soc. Am. 44, 359(A), 1968] according to which $\sigma$ is a joint property of the room and the source spectrum. A progress report is given, and measurements taken in one-third octave bands are shown to be in reasconable agreement with this theory. 


\title{
Session AA. Engineering Acoustics III: Underwater Transducers
}

\author{
Bart N. Locanthi, Chairperson \\ 2180 Pinecrest Drive, Altadena, California 91001
}

\section{Contributed Papers}

$2: 00$

AA1. Electro-optic hydrophone. S. Hanish (Naval Research Laboratory, Wasbington, DC 20375) and J. Chovan (General Electric Co. , Syracuse, NY)

The electro-optic hydrophone is a device that detects sound in water by scattering a laser beam off naturally occurring particles moving under the influence of acoustic signals. The theory of operation is the same as that of $\mathrm{cw}$ Doppler radar or (alternatively) of an optical heterodyne in which the laser beam is split into two beams to form a set of real (or virtual) fringes at the focal volume in the water [Rudd, J. Sci. Inst. 2, 55 (1969) ]. Moving particles traverse these fringes and are intermittently illuminated. A photodetector converts the scattered light intensity into an intermittent electric current which is processed in a power spectrum analyzer. A laboratory experiment is described in which an acoustic signal at $200 \mathrm{~Hz}$ and peak amplitude of $4.35 \times 10(-8) \mathrm{m}$ is detected by this principle. A discussion is then made of the minimum detectable signal of this device in the presence of noise from various origins. Methods are proposed to lower the threshold of detection from the level curr ently achieved.

$$
2: 15
$$

AA2. Rare earth-iron magnetostrictive underwater sound transducer. S.W. Meeks and R.W. Timme (Underwater Sound Reference Division, Naval Research Laboratory, P. O. Box 8337, Orlando, FL 32806)

A piston-type $k_{33}$-mode underwater sound transducer utilizing rods of $27 \%$-terbium- $73 \%$-dysprosium iron alloy as the transduction element is described. The broadband frequency operation permits intercomparison of the terbium-dysprosium iron and ceramic element, as well as comparison with predictions based on basic material property measurements. An equivalent circuit which successfully describes the transducer performance and data on free-field voltage sensitivity, transmitting current response, coupling coefficient, directivity, efficiency, linearity, and power drive are presented. Predictions based upon the equivalent circuit for the performance of a laminated transducer are discussed. Design problem areas resulting from the requirement of large magnetic fields, eddy current losses, and alloy brittleness are discussed and compared to design problems encountered with ceramic transduction materials.

$$
2: 30
$$

A A3. PHD-low-frequency transducer. C. S. Nichols (Code 3022, Naval Undersea Center, San Diego, CA 92132)

The area of low-frequency sound sources has received considerable emphasis in the past several years. Of particular interest is the development of small, lightweight low-frequency transducers that have broadband capability. One particular low-frequency transducer that fits into this category is the PHD (post hole digger). The PHD is cylindrical in shape with a diameter of $0.3048 \mathrm{~m}$, a length of $0.4572 \mathrm{~m}$ and has an in-air weight of $36.3 \mathrm{~kg}$. The driving mechanism for the PHD is a transverse variable reluctance magnetic motor. The unit must be pressure compensated and requires large input currents (15-30 amp) below $30 \mathrm{~Hz}$. The PHD has recently been tested at Lake Pend Oreille to a depth of $61 \mathrm{~m}$. The response of the unit was measured as a function of operating depth dur ing the Pend Oreille tests. At the $61 \mathrm{~m}$ depth, the maximum source level that it was possible to obtain was $168 \mathrm{~dB}$ re $1 \mu \mathrm{Pa}$ at $1 \mathrm{~m}$. This limit was imposed by the amplifiers used during the tests and is not a limit of the transducer. [Work supported by NAVSEA 06H1. ]

$$
2: 45
$$

AA4. Test report of Westinghouse LB 16 low-frequency flexural disk transducer at NUC Calibration Facility, Lake Pend O'Reille, Idaho. C.S. Nichols (Naval Undersea Center, San Diego, CA 92132) and F. Tighe, Jr. (Westinghouse Electric Corporation, Defense and Electronic Systems Center, P.O. Box 1897, Mail Stop 687, Baltimore, MD 21203)

Flexural disk transducers are used as underwater sources for low-frequency sound. The large displacements needed for low-frequency sound generation requires that the backing material for the radiating disks be very compliant. The radiating disks are quite compliant and are susceptible to breakage when a pressure differential exists between the radiating face and the backing face of the disks. Hence for underwater applications flexural disks are usually pressure compensated to keep the pressure in the backing material equal to the ambient hydrostatic pressure. a passive pressure compensation system has been developed and incorporated into a 16-in.-diam. doubletrilaminar flexural disk. The transducer system has been tested at Lake Pend O'Reille, Idaho, by Naval Undersea Center and Westinghouse Electric Corporation.

$$
3: 00
$$

AA5. Optimally wide-band lightweight flextensional transducer. G. Brigham (2543 Ames Ave., Anaheim, CA 92806)

An experimental Class IV Flextensional transducer consisting of three identical air-backed modules, built and tested in 1969 at the Autonetics Division of Rockwell International, generated a $40 \mathrm{~dB} / \mathrm{V}$ (re $1 \mu \mathrm{bar}$ at $1 \mathrm{yd}$ ) source level over a $3-\mathrm{dB}$ bandwidth of $1.7-6.5 \mathrm{kHz}$. From a detailed analysis of this type of transducer it appears the use of glass reinforced plastic oval shells to replace the aluminum shells, stagger tuned modules, and compliant coupling of ceramic bar to shell would result in an experimental transducer of lighter weight and much larger bandwidth than the previous unit. The acoustic effects of an oil filling used for pressure compensation to depths in excess of five hundred feet but less than about 2000 $\mathrm{ft}$ in the ocean can be at least partially offset by use of HY300 steel psuedocompliant tubes molded into the inner walls of the GRP shells. This paper presents the performance characteristics, weight, and geometry of the older unit and discusses the basic design features of this type of transducer.

$$
3: 15
$$

AA6. Development of a cost effective $65-\mathrm{kHz}$ tonpilz element. F. Tighe, Jr. and G. R. Douglas (Westinghouse Electric Corporation, Mail Stop 687, Baltimore, MD 21203)

A $65-\mathrm{kHz}$ tonpilz element was designed, constructed, and calibrated. The element is to be used as a pump array for an underwater parametric receiving system. To reduce the cost of the array, the component parts and $Q$ were minimized and the radiation-head size was maximized. In order to avoid head 
flexure, the size of square radiation heads to tonpilz elements are typically less than $0.7 \lambda$. The element in this report has a head $1.35 \lambda$ square, reducing the number of elements for a given aperture by a ratio of $3.7: 1$. The measured $3-\mathrm{dB}$ bandwidth was approximately $16 \mathrm{kHz}$, eentered at $65 \mathrm{kHz}$, representing a $Q$ of 4 .

$$
3: 30
$$

AA7. Design of high-efficiency transducer elements and arrays. M.W. Widener (Applied Research Laboratories, The University of Texas at Austin, P.O. Box 8029, Austin, TX 78712)

Parametrie sound reception has created a need for pump transdueers capable of continuous operation with intense sound fields. Reduction of internal losses and improved heat transfer become important design goals. A transducer structure has been developed in which the internal mechanical energy dissi- pation is reduced dramatically and the thermal coupling of the ceramic to the water is improved. The basic design consists of a quarter-wavelength mass directly immersed in the fluid medium with the ceramic element surrounded by an air cavity and driven in quarter-wavelength resonance. The assembly is suspended at the vibrational node by a metal flange formed integrally with the radiating mass. The resonant $Q$ of the unloaded assembly in air can be as high as $300-400$. Water loading diminishes the eircuit $Q$ to the order to $5-15$ which corresponds to an effective electrical-to-acoustic energy conversion efficiency in the range of $85-98 \%$. Practical elements exhibiting the same general quality of operation have been constructed over the frequency range from 7 to $400 \mathrm{kHz}$. [This research was supported by the Advanced Research Projects Agency of the Department of Defense and was monitored by the Naval Electronic Systems Command under Contract N0003976-C-0231.1

WEDNESDAY, 17 NOVEMBER 1976

\title{
Session BB. Shock and Vibration III: Shock in Ships
}

\author{
Harry L. Rich, Chairperson \\ 6765 Brigadoon Drive, Bethesda, Maryland 20034
}

Invited Papers

$2: 00$

BB1. History, development, and resources for ship shock hardening. Henry C. Pusey (Shoek and Vibration Information Center, Code 8404, Naval Research Laboratory, Washington, DC 20375)

This paper will cover the historical development of programs designed to increase the survivability of naval ships to shock generated by various sources such as eontact and noncontact underwater explosions, impact of projectiles and bombs, firing of the ships guas and other weapons, and air blast. The historical development will touch upon technical advances from World War I to the present in the areas of defining the shock threat, the evolution of design techniques, the methods and desirability of shock isolation, the changing specifications, and the improvements in acceptance methods involving both test and analysis. Finally, the presentation will include a eomprehensive description with problems involving the hardening of ship structures and equipment to shock from the various sources.

$$
2: 30
$$

BB2. Shipboard shock environment and its measurement. M. W. Oleson and R. O. Belsheim (Ocean Technology Division, Naval Research Laboratory, Washington, DC 20375)

The design and acceptance of machinery and equipment for shipboard use requires knowledge of the shock environment produced by noncontact underwater explosions. Structural and response considerations preclude accurate prediction of this environment without $\epsilon$ xperimental confirmation. The Navy has conducted many experiments to measure shipboard shock environment and its effect on internal items. This data and results from dynamic analyses yield a general understanding of the shipboard shock environment, but this understanding is restricted to ships resembling those for which experimental data exists. This paper discusses the varled types of shock environment which may be expected on small and large ships. An important factor for response studies is the dynamic interaction between a machine and the ship structure. The kinds of shock measurements and the means of interpreting; these data are outlined and the results of several underwater-explosion test series against operating ships are summarized. These data are discussed in terms of the types of shock environment and the interpretive analyses of the shock measurements. A concluding section reviews capabilities for predicting shock environment of present and variant ship designs. 
$3: 00$

BB3. Options and techniques for shock hardening of ship's equipment. Robert M, Mains (Department of Civil Engineering, Washington University, St. Louis. MO 631:31)

The problem of shock hardening ship's equipment is discussed from initial planning and strategy development through final action decision after design, analysis, and test. The planning operation is the one in which performance criteria are established, the design is anticipated, the analysis is laid out, and the designer is given a means for developing a preliminary design. With the preliminary design in hand, the static structural calculations are performed and the mass and stiffness matrices are assembled. With the normal modes and frequencies from an eigenvalue run, the system displacement responses to step-velocity shock are obtained. These responses can be converted to member forces and stresses or accelerations. The results are evaluated against the performance criteria, and the whole process is reexamined for validity before action decisions are made.

$$
3: 30
$$

BB4. Simulation of shipboard shock and use for experimental validation. B.C. Gartman and N. Elmore, Jr. (Structures Department of the David Taylor Naval Ship Research and Development Center, Bethesda, MD 20084)

Abstract not available.

$$
4: 00
$$

BB5. U。S. Navy shock hardening program and philosophy. Jerry Sullivan (NAVSEA Naval Ship Engineering Center, Hyattsville, MD)

Abstract not available.

$$
4: 30
$$

BB6. Dynamic considerations in the design of floating nuclear power plants (FNPP)。 $G_{a} G$. Amir (824 New Mark Espl. Rockville, MD 20850) and K. K. Kapur (U.S. Nuclear Regulatory Commission, Washington, DC)

Design criteria and their implementation in the FNPP when subjected to dynamic effects are discussed. The FNPP idea was proposed in the late $1960^{\prime}$ 's because of site flexibility, immediate abundance of water, possible decoupling from seismic shock, and because the concept lends itself to a high level of standardization. The FNPP, when located offshore, must be protected by a breakwater to which it is permanently fastened by a mooring system. The dynamic considerations affecting the design of the FNPP are due to natural phenomena, accidents, and man-made adverse conditions. Tornadoes, hurricanes, tsunami waves, and ice loading are considered for both safe operation and safe shutdown of the plant. Earthquakes, sub-marine slides, and underwater currents are considered for the breakwater design as well as for their effect on the FNPP through the mooring system. The above conditions are investigated by model test and analysis of the seabed-fluid-structure interaction. Accident conditions such as ship collisions with the breakwater, shipping accidents resulting in air blast, and aircraft crash are examined by statistical and probability methods and by structural testing and analysis. 


\title{
Session CC. Speech Communication IV: Speech Production: Anatomy and Physiology. Precis-Poster Session
}

\author{
Osamu Fujimura, Chairperson
}

Bell Laboratories, Murray Hill, New Jersey 07974

\section{Poster Session}

$2: 00$

CC1. The geniohyoid and the role of the strap muscles in pitch control. Donna M. Erickson (Haskins Laboratories, 270 Crown St., New Haven, CT 06510), Mark Y. Liberman (Bell Laboratories, Murray Hill NJ 07974), and Seiji Niimi (Haskins Laboratories, 270 Crown St., New Haven, CT 06510)

Many investigators have noted a relationship between strapmuscle activity and pitch lowering, but there does not seem to be any single generally accepted theory to account for this connection. The articularly effect of strap-muscle contraction will depend in part on what other forces are acting on the hyoid bone; therefore, in the context of a general EMG investigation of English intonation, we recorded from a supraphyoidal muscle, the geniohyoid. In our data, the three strap muscles show nearly identical patterns of activity; as a group, their activity shows a strong negative correlation with the activity of the geniohyoid. By comparing these patterns of activity for otherwise identical utterances with systematically varied pitch contours, we are able to sort out the effects resulting from tongue and jaw movements from the activity specifically related to pitch control, and to arrive at an hypothesis concerning the effects on vocal-cord tension of the forces applied, by these extrinsic muscles, to the hyoid bond and the thyroid cartilage.

\section{$2: 04$}

CC2. Lip larynx interaction models for intervocalic /p/ production. Anne H. B. Putnam (Speech Research Laboratory 1510, Veterans Administration Hospital, 4150 Clement St. , San . Francisco, CA 94121), E. Thomas Doherty (IASCP, University of Florida, Gainesville, FL 32611), and Thomas Shipp (Speech Research Veterans Administration Hospital, San Francisco, CA 94121)

Simultaneous recordings of labial and laryngeal EMG, subglottal and intraoral pressures, and voice were obtained from six talkers to study lip/larynx interaction during production of intervocalic $/ \mathrm{p} /$ in three talking conditions: subjects spoke normally, with the jaw fixed, and with a transoral pressurerelease device. Schematic models were used to categorize the temporal and magnitude details of the sequence of articulatory events for $/ p /$ in each condition. Multivar iate regression analyses isolated those differences between control and experimental conditions which implied a significant change in motor programming for $/ \mathrm{p} /$ when normal lip/larynx articulation was modified experimentally. Treatment of the data thus far reveals that when the jaw is fixed, significant readjustment for production of $/ p /$ occurs only in lip-muscle activity patterns. For the pressure-release-device condition, aerodynamic, and voicing patterns for $/ \mathrm{p} /$ are altered when intraoral pressure is unexpectedly released, but preliminary statistical analysis reveals no significant concomitant readjustment in laryngealmuscle activity. [Work supported by NII and VA Research Service. I

\section{$2: 08$}

CC3. EMG study of the coarticulation of lip rounding. F. Bell-Berti (Montclair State College, Upper Montclair, NJ 07043, and Haskins Laboratories, 270 Crown St. , New Haven, CT 06510) and K.S. Harris (The Graduate School, The City
University of New York, New York, NY 10036, and Haskins Laboratories, 270 Crown St., New Haven, CT 06510)

The extent of both anticipatory and sarryover coarticulation is of central importance to theories of speech organization. One articulatory gesture whose anticioation has been studied repeatedly is vowel lip rounding. Daniloff and Moll [J. Speech Hear. Res. 11, 707-721 (1968) ], reporting the results of a cinefluorographic study of lip roundins, describe vowel lip rounding as being initiated with the beginning of a preceding consonant string, for strings up to four segments long. Preliminary results of an EMG experiment in which /u/ was preceded by a one- or two-consonant string show activity for the rounding gesture beginning at the same time before the vowel onset for all conditions regardless of the position of the syllable boundary. Parallel results were obtained for carryover coarticulation.

\section{$2: 12$}

CC4. Vocal-tract responses to unexpected, suddenly induced decreases in intraoral air pressure. Joseph S. Perkell (Research Laboratory of Electronics, Masssachusetts Institute of Technology, Cambridge, MA 02139) and Thomas Gay (University of Connecticut Health Center, Farmington, C'T 06032 and Haskins Laboratories, New Haven, CT 06510)

In a pilot study [J.S. Perkell, J. Aizoust. Soc. Am. 58, S12 (A) (1975) ] a vacuum apparatus was used to create suddenly induced, unexpected decreases in intraoral air pressure (suction) during prestressed intervocalic / $\mathrm{p} /$ production. It was found that with labial anesthesia, VOT measured from the moment of consonant onset occurred $\mathrm{c} t \mathrm{n}$ average of $20 \mathrm{msec}$ later during suction trials than during normal intraoral air pressure trials. In order to explore the possible relationship of this effect to the function of feedback mechanisms, the exper iment is being elaborated. Electromyographic measure-ments are being made in conjunction with variation in the depth of labial anesthesia and timing of the onset and offset of the "suction" impulse. Preliminary results will be discussed. [Research supported by NIH grants.]

$$
2: 16
$$

CC 5. Laryngeal timing constraints in plosive-vowel and fricative-vowel syllables, or: Is VOT really the best measure of glottal-supraglottal timing? T.M. Klee, G. Weismer, and D. R. Ingrisano (Indiana University, Speech and Hearing Center, Bloomington, IN 47401)

Voice-onset time (VOT) is generally defined as the time interval between release of an occlusive consonant and the onset of vocal-cord vibration. This description, however, does not allow a simple comparison of glottal-isupraglottal timing between syllables initiated with stop and fricative manners of articulation, since the time of release associated with fricative is less well defined than that associated with stops. We compare glottal-supraglottal timing across consonant manner of articulation by normalizing the duration of selected target utterances spoken in a sentence frame by 15 subjects, and deriving a measure which expresses the timing of glottal-supraglottal events relative to the duration of a potential unit of articulatory encoding, viz., the syllable. Additional motivation for developing an alternative measure to VOT comes from 
preliminary data reported by Umeda and Coker (J. Phonetics $2,1-5(1974)]$, which suggests that stop closure duration and VOT are not independent in connected speech. We discuss our results relative to the aerodynamics of consonant production, and the potential limitations of the VOT measure as currently defined.

$$
2: 20
$$

CC6. Magnitude of perioral reflexes as a function of stimulus velocity and isometric lip force. Charles R. Larson, John W. Folkins, Mike McClean, and Eric M. Muller (Department of Speech and Hearing Sciences, University of Washington, Seattle, WA 98195)

A quantitative description of peripheral nervous-system function may enhance our ability to analy ze speech motor control. With respect to lip motor control, recent evidence [R. Netsell and J. Abbs, J. Acoust. Soc. Am. 68, S41 (1975)] suggests that perioral reflexes may be actively modulated throughout lip movement for speech. In the present study, factors explored to clarify the manner in which these reflexes may be modulated during control of lip movement. Previous studies of perioral reflexes have been handicapped by the inability to systematically vary the characteristics of the mechanical simulus. The present investigation utilized an electromagnet under displacement sensitive servo control, coupled to a force transduction system, which delivered mechanical stimuli of different velocities against the lip of normal adult subjects. Reflexes were recorded from hooked-wire EMG electrodes inserted into the orbicularis oris muscle while the lip was either passive or exerting an isometric force. Perioral reflexes were found to increase in magnitude as a function of both stimulus velocity and the level of isometric force. [Work supported by NIH.]

$$
2: 24
$$

CC7. Oscillatory movements of the velum: A factor of mandibular and/or intraoral air-pr essure variation. W.N. Williams (College of Dentistry, University of Florida, Gainesville FL 32611), W. S. Brown, Jr. (Department of Speech, University of Florida, Gainesville, FL 32611) and B. Hilderbrand (University of Texas, Dallas, TX 75230)

It is known that velar elevation in closure for speech correlates with specific sound productions. In addition, the velum has been observed to oscillate during repeated syllable or word utterances. The purpose of this study was to determine whether this oscillatory movement of the soft palate may be related to the build up and release of intraoral air pressure and/or to the opening and closing patterns of the mandible. Simultaneous cinefluorogr aphic films and air-pressure recordings were taken for three subjects during the repeated production of /apa/, /aba/, /asa/, and /aza/. From the cinefluorographic films, variations in velar and mandibular height were determined and correlated with variations of air pressure. Initial analyses Indicated that there was no clear relationship between either variation in air-pressure or vertical displacement of the mandible with oscillatory motions of the velum. These results will be discussed in terms of a theory/model of articulatory behavior for oral consonant production.

\section{$2: 28$}

CC8. Syllable-final nasal elements in English-an x-ray study of velum height. O. Fujimura and J. E. Miller (Bell Laboratories, Murray Hill, NJ 07974) and S. Kiritani (Faculty of Medicine, University of Tokyo, Hongo, Tokyo, Japan)

An English syllable can contain, in the final part, a nasal consonant either as the main consonant with place specification as in "den," "denned," etc. , or with another true consonant within the syllable core, as in "dent," etc. [o. Fujimura, J. Acoust. Soc. Am. 59, Z5 (A) (1976)]. A set of English monosyllabic words containing various syllable-final clusters were uttered by a native speaker of American English, and the movements of the velum as well as three selected points on the tongue, a lower tooth, and the lower lip were tracked by means of the computer-controlled x-ray microbeam sys- tem [S. Kiritani, K. Itoh, and O. Fujimura, J. Acoust. Soc. Am. 57, 1516-1520 (1975)]. The data contained about 300 such word utterances as well as several sentences, and have been examined with particular reference to the above-mentioned distinction in terms of exact articulatory characteristics. The results will be discussed also in comparison with previous findings about Japanese nasal consonants in different intrasyllabic positions [O. Fujimura, J.E. Miller, and S. Kiritani, J. Acoust. Soc. Am. 58, V5 (A) (1975) ].

$$
2: 32
$$

CC9. Stereo fiberscopic investigation of the larynx. Seiji Niimi and Thomas Baer (Haskins Laboratories, New Haven, CT 06510) and Osamu Fujimura (Bell Laboratories, Murray Hill, NJ 07974)

Present fiberoptic laryngoscopic techniques [Sawashima and Hirose, J. Acoust. Soc. Am. 43, 168-169 (1968) ] are limited because lens-to-object distance cannot be determined. To overcome this limitation, a laryngeal ster eo fiberscope, which can supply a side-by-side pair of images within each film frame, has been developed. The fiberscope comprises two independent fiberoptic systems joined to a common viewing and control unit. The two fiberoptic bundles are inserted to the level of the oral pharynx through a subject's two nostrils, then their tips are fixed to a rigid connector, and they are directed toward the larynx. The three-dimensional position of a point in the object field can be determined from measurements of its position in the two images. Measurements of several points on the larynx can be used to determine its vertical position, its orientation (tilt), and its actual dimensions. Measurement error in the vertical direction depends on several factors, but under typical conditions calibration has given a maximum error range of $\pm 5 \%$. Preliminary measurements of laryngeal movements during phonatory and respiratory gestures will be discussed.

\section{$2: 36$}

CC10. Stresses in vocal-fold tissues during normal phonation. Ingo R. Titze (Sensory Communication Research Laboratory, Gallaudet College, Washington, DC 20002)

In order to predict the occurrence of pathologic conditions in the vocal-fold tissues resulting from abnormal use and abuse, a theoretical study of various shear and compressional stresses under normal phonation has been attempted. Results are based on a continuum as well as a finite element of model of the vocal folds. These models differ from previous ones in that distributive mechanical properties, i.e. , shear elasticity and viscosity, are used to describe the dynamics. It is shown that near glottal closure the stresses at the medial boundary of the vocal folds resemble a doublet, i.e., a sharp negative stress resulting from the short range Bernoulli forces followed by an immediate positive stress after closure resulting from vocal fold collision. The peak to peak magnitude of this doublet can be of the order of $10^{5}-10^{6} \mathrm{dyn} / \mathrm{cm}^{2}$ and occurs in a time interval of the order of $10^{-4}$ sec. Such an impulse could easily contribute to permanent tissue modification, and the formation of pathologies such as contact ulcers and vocal nodules. Modes of phonation which may avoid such a high stress concentration are discussed.

$$
2: 40
$$

CC11. Oral pressure and laryngeal control. William G. Ewan (Department of Oral Blology, University of Connecticut Health Center, Farmington, CT 06032)

It is not clear from studies of larynx movement during stop production that the larynx is perturbed up for voiceless stops or down for voiced stops. One would expect it is the latter, however, since larynx lowering would provide a slight increase in oral cavity volume and would thus help to prolong the pressure difference across the glottis necessary for voicing. A test was made to see whether subjects during continuous phonation would lower the larynx in response to an artificially induced oral pressure buildup. Three subjects were used. 
Vertical larynx movement was recorded using the thyroumbrometer while a valving device inserted in the subject's mouth simulated an oral closure at random intervals. Valve closure resulted in a significant lowering for all three subjects. The fastest response times (the time between valve closure and the beginning of larynx lower ing) approached the lower limits of motor response times associated with voluntary movement. The results indicate the larynx can be lowered during stops in order to prolong phonation. However, the magnitude of this delay in effecting this is large enough to suggest that if a similar lowering of the larynx occurs on voiced stops during speech, it must be an active preprogrammed gesture, not a passive compensatory response to oral pressure build-up. IThis research was supported by a NSF grant to the Phonology Laboratory, University of California. Berkeley, and by a NIDR postdoctoral fellowship at the Department of Oral Biology, University of Connecticut Health Center, Farmington, CT.I

\section{$2: 44$}

CC12. Determination of glottal area based on digital image processing of high-speed motion pictures of the vocal folds. S. B. Davis (Speech Communications Research Laboratory, Ine., 800 A Miramonte Dr. , Santa Barbara, CA 93109)

The extraction of glottal-area data using frame-by-frame analysis of high-speed motion pictures of the vocal folds has previously been a time-consuming process requiring manual interaction. Completely automatic extraction of glottal-area data may be realized by two-dimensional digitization of sequential frames of high-speed film followed by computer algor ithms for edge detection and area calculation. In a recent feasibility study, six photographs ( 2.56 in. $\times 2.56$ in. each) depicting successive discrete movements of the vocal folds during the open phase of one pitch period were digitized (512 points $\times 512$ points $\times 256$ gray levels each) and stored. Brightness, contrast, and normalization algorithms were utilized to compare the observed detail in the digital representations with the original data. The glottal area of each frame was automatically calculated from the digitized images with no manual intervention, and the areas were compared with planimeter measurements for accuracy. The results indicate that it is possible to apply digital image processing techniques to accurately display physiological details and to automatically extract the glottal area from high-speed motion pictures of the vocal folds.

\section{$2: 48$}

CC13. The function of strap muscles in speech: pitch lowering or jaw opening. James E. Atkinson (Naval Underwater Systems Center, New London, CT 06320) and Donna Erickson (Haskins Laboratories, New Haven, CT 06510)

This paper reports on one aspect of a continuing study to determine the physiological correlates of the changes in fundamental voice frequency $\left(F_{0}\right)$. Severa! electromyographic (EMG) studies with speech have reported an association of strapmuscle activity, particularly the sternohyoid, with low $F_{0}$ and some of these studies suggest that the sternohyoid is actively involved in lowering $F_{0}$. It has also been suggested, however, that the sternohoid is involved with jaw opening, and that the reported pitch-lowering effects may actually be the result of jaw opening. To investigate this question an EMG experiment was conducted on one speaker of American English under normal and clenched jaw conditions. The normal utterances were of the form "Bev loves Bob" with emphasis on the various words. The clenched-jaw data were obtained while the subject held his jaw fixed by biting on a tongue depressor and intoned the corresponding intonation patterns with a fixed vowel carrier $/ a /$. The results indicate that the strap muscle activity for the normal utterances is very similar to the activity for the same intonation pattern with the jaw clenched. Strap-muscle activity thus seems to be more closely related to pitch effects than to jaw-opening effects.

\section{$2: 52$}

CC14. Effects of subglottal pressure changes on sustained phonation. Thomas Baer (Haskins Laboratories, 270 Crown St. , New Haven, CT 06510)

The intrinsic dependence of fundamental frequency of phonation on subglottal pressure has been studied in several investigations by producing involuntary changes in pressure. In some of these experiments, the pressure changes were produced by suddenly pressing at random intervals on the chest of a phonating subject. Nuscle activity was assumed constant in the first few pitch periods immediately following the beginning of pressure rise. In the present irvestigation, the chest-pushing paradigm was used while both subglottal pressure and the electromyographic activit $/$ of several laryngeal muscles were recorded. In a preliminc.ry experiment, fundamental frequeney was found to vary with subglottal pressure at average rates of $3-5 \mathrm{~Hz} / \mathrm{cm} \mathrm{H}_{2} \mathrm{O}$. However, electromyographic responses of some intrinsic laryngeal muscles were found to begin very shortly after the beginning of the pressure rise. Average delays for the vocalis muscle for three conditions ranged from 0 to $30 \mathrm{msec}$. Variations in vocal intens ity more closely paralleled the changes in pressure than did fundamental frequency. Implications of these results will be discussed.

$$
\text { 2: } 56
$$

CC15. Coordination of consonant and vowel articulations in Japanese. S. Kiritani, H. Hirose, and M. Sawashima (Research Institute of Logopedics and Phoniatrics, Tokyo University, Hongo 7-3-1, Tokyo, Japan)

By means of pellet-tracking technique using an x-ray microbeam system, observations of articulalory movements for a larget set of Japanese nonsense words containing various types of $\mathrm{CV}$ syllables, vowel sequences have been conducted. In particular, character istics of mutual influences of the vowel and consonant articulations upon each other were investigated. It was observed that (1) in $V_{1} C V_{2}$ articulations, relative timing of consonant articulations and $V_{1}-V_{2}$ transitions varied depending on the type of consonant; and (2) for the vowel articulation in $\mathrm{CVC}$, the degree of perturbation of tongue tip position for $/ \mathrm{t} /$, tongue body position for $/ \mathrm{k} /$, and jaw position for $/ \mathrm{p} /$ was approximately same, wher eas the perturbation of lip position for $/ p /$ was smaller than that of jaw po.sition. Coordinations of the movements of the velum, tongue, and lip were also investigated with special reference to the difference in the syllable initial and syllable-final nasal consonants in Japanese. Basic set of data on English is also being studied for comparing articulatory characteristics of English with those of Japanese.

\section{$3: 00$}

CC16. Repeatability of articulatory movements. T. Gay (Department of Oral Biology, University of Connecticut Health Center, Farmington, CT 06032) and Haskins Laboratories, New Haven, CT 06510) and S. Kir itani and H. Hirose (Research Institute of Logopedics and Phoniatrics, Faculty of Medicine, University of Tokyo, Tokyo, Japan)

In this experiment, we studied the repeatability of articulatory movements using the computer controlled $x-r a y$ microbeam system at the University of Tokyc. Small lead pellets were attached to the upper lip, lower lip, lower central incisor, tongue tip, tongue body, and a reference location on the upper central incisor of each of two adult male subjects, both native speakers of American English. Each speaker read a randomized list of six different nonsense syllables and two sentences containing the vowels, $/ i, a, u /$ and the consonants, $/ p, t, k /$ in various VCV combinations, 15 tImes at a controlled speaking rate. The movements of the pellets wer e tracked at a rate of 150 scans/sec and recorded in graphical form with the $X$ and $Y$ components plotted separately against time. Spectrograms were obtained from recordings of the corresponding acoustic signal. Both sets of data are used to explore the questions of articulatory and acoustic equivalence in speech production. 
3:04

CC17. Recruitment and discharge patterns of single motor units during speech production, H. M. Sussman, R. K. Powers . P. F. MacNeilage (Department of Linguistics, Univer sity of Texas, Austin, TX 78712)

Study of single motor unit (SMU) activity during rapid isotonic contractions, such as those characterizing speech. provide a closer look at the inacessible central mechanisms responsible for the encoding of the speech motor program. This study represents a quantification of the discharge and recruitment patterns of SMU's in the anterior belly of digastric, a muscle active in bringing about jaw lowering. Using a highly selective intramuscular electrode and computerized analysis procedures the firing rate, recruitment intervals, and various temporal intervals between SMU activity and articulatory events were obtained. Results to be discussed include: (1) greater isotonic versus isometric discharge rates for the same motor unit; (2) higher maximum discharge rates for later and larger recruited motor units; (3) relative invariance of recruitment order; (4) temporal sequence of SMU activation in relation to initiation of jaw lowering and speech audio signal: and (5) relationship of SMU activity to articulatory displacement and velocity. Implications of these results towards a further understanding of the encoding mechanisms of speech will be discussed.

\section{$3: 08$}

CC18. Persistence of learned motor patterns in speech. $S$. Hamlet, M. Stone, and T. MeCarthy (Department of Hearing and Speech Sciences, University of Maryland, College Park, MD 20742)

It has been noted that "spontaneous" compensations are made during speech for articulatory constraints such as clenching of the jaw. On the other hand, at least several days are required for speech adaptation to a dental prosthesis. A recording of speech and physiological data on jaw activity and tongue contacts was made at the time of initial insertion of an experimental dental prosthesis. Subjects wore the prothesis for two weeks of adaptation, after which a second recording was made, and then waited at least 1 month without experience in speaking with it. A follow-up recording was then made with the same protocol as when the prosthes is was first inserted. Speech sounded much less disturbed by the insertion of the prothesis after a lapse of a month than it had at initial insertion-knowledge of the compensatory adjustments required had evidently been retained over that period of time. Palatographic data showed a rapid progression toward the compensatory pattern within a few repetitions of the speech sample. [Work supported by NIH.]
$3: 12$

CC19. Voluntary reaction times for phonation initiation. Krzysztof Izdebski and Thomas Shipp (Speech Research Laboratory, Veterans Administration Hospital, 4150 Clement St. , Sạn Francisco, CA 94121)

Simple neuromechanical reaction times for voluntary initiation of phonation were investigated in 15 males and $15 \mathrm{fe}-$ males as a function of (1) stimulus type: either a $1-\mathrm{kHz}$ tone or a drop from $6 \mathrm{~cm} \mathrm{H} \mathrm{H}_{2} \mathrm{O}$ in intraoral air pressure to atmospheric pressure; (2) abducted, adducted, or uncontrolled prephonatory vocal-fold position and $(3) 75 \%, 50 \%$, and $25 \%$ of subject's lung volume. Auditory-manual responses were also obtained from these same subjects. All responses were to stimulus onset. The fastest neuromechanical reaction times were $120 \mathrm{msec}$; however, the average time for phonation initiation across subjects was $180 \mathrm{msec}$. Auditory-vocal reaction times were generally faster than somesthetic-vocal reaction times, but these differences were not significant. Faster auditory-vocal reaction times were obtained at mid than at lung volume extremes, as well as when phonation was initiated from abducted rather than adducted prephonatory vocal-fold positions. Manual reaction times were significantly faster than vocal reaction times,

$$
3: 16
$$

CC20. Language rhythms as assimilated rhythms in the nervous system. Warren Creel (Albany Medical College, Albany, NY 12208) and Paul C. Boomsliter (State University of NY at Albany, Albany, NY 12222)

Electronic studies of neural activity have shown that it is characteristic of brains to assimilate a rhythm from an incoming stimulus, and to maintain an assimilated rhythm through gaps. (E. R. John, Mechanisms of Memory (Academic, New York, 1967), especially pp. 294-343]. In music, a "rest" is a beat supplied by an assimilated rhythm. Rhythmic rests play a role in the typical structure of children's rhymes. A cross-linguistic study of children's rhymes in such unrelated languages as English, Chinese, Arabic, Nigerian, Southern California Indian, etc. , finds the same systematic pattern predominating in all, although cultural borrowing is excluded. This indicates they are shaped to eater to processes that come naturally to the nervous system. IRobbins Burling, "The metrics of children's ver ses : a cross-linguistic study," American Anthropologist, Vol. 68, No. 6, Pt. 2 (Dec. 1966) pp. 1418-1441]. Instances of rests and filled rests, shown by measurements of English verse, demonstrate that the nervous system makes a basic contribution to rhythm patterns, so that in analyzing them we should not think of the rhythm as continually driven by the stimulus. 


\title{
Session DD. Noise V: Response to Noise
}

\author{
William K. Connor, Chairperson \\ Tracor, Inc., Austin, Texas 78721
}

\section{Contributed Papers}

$$
\text { 4:15 }
$$

DD1. Individual differences in temporal integration weightings for the annoyance of sets of noises. A Ahumada, Jr. (Department of Aeronautics and Astronautics, Stanford University, Stanford, CA 94305) and David C. Nagel (National Aeronautics and Space Administration, Ames Research Center, Moffett Field, CA 94035)

Observers rated the overall annoyance of a group of three brief bursts of noise spaced $15 \mathrm{sec}$ apart. The levels of the bursts within each group were one of three values, 60,75 , and $90 \mathrm{~dB} A$, yielding 27 stimulus groups. Each stimulus was played and rated in the presence of three backgrounds-a noisy street, a playground, and a quiet park. A three mode factor analysis of the ratings was performed using the canonical decomposition option of the Carroll and Chang INDSCAL program. The analysis finds weights for observers, background and stimuli which give the best least squares fit to the ratings. This scaling method was used because it gives a configuration with uniquely determined dimension directions. The best solution has three dimensions. The dimension accounting for the most variance $(45 \%)$ represents loudness integrated independently of time. The second dimension represents loudness integrated with a sharply decaying time constant (so that the level of the most recent noise dominates). The third dimension accounts for variance introduced by changes in the background level. Observer weights on the dimensions indicate the manner in which individuals differ in arriving at the overall annoyance judgments. [Work supported by StanfordAmes Joint Institute for Aeronautics and Acoustics. ]

$$
\text { 4:30 }
$$

DD2. Hearing damage from forest firefighting operations. R.T. Harrison (USDA Forest Service, 444 E. Bonita Ave., San Dimas, CA 91773) and R.B. Chaney, Jr. (Department of
Communication Seience and Disorders, University of Montana, Missoula, MT 59801)

Audiological examination of Forest Service "Helitack" personnel (firefighters who are transported directly from their base to the fire scene by helicopters) reveals that a large percentage of them show significant hearing loss characteristic of noise-induced PTS. There are indications that this PTS is correlated with length of service on helicopters. However, measurements of the helico ter noise environment and of normally encountered exposure times indicate that OSHA noise exposure criteria are not exceeded. Also, for a limited sample of normal hearing helitack personnel, no TTS was observed after a 40-min flight. T'vo possible explanations for the unexpectedly high incidence of PTS are discussed in this paper. The first is that activities ather than helicopter operations cause the PTS. This is investigated by means of a noise-history questionnaire. The second is that OSHA criteria are not stringent enough for these particular operations. Finally, the program that the Forest service is implementing for helitack crews is briefly discussed.

$\checkmark$

\section{4:45}

DD3. Reaction of artic wildlife to gas pipeline related noise. J.S. Ruth (Engineering Dynamics, Inc., 6651 S. Wellington Ct. Littleton, CO 80121).

Several studies on the reaction of aretic animals to noise emissions from gas compressors and aircraft are reviewed. Wildlife species examined include caribou, Dall sheep, moose, grizzly bears, wolves, snow geese, Lapland longspurs, and bald eagles. Degree of reaction to noise disturbance was found to vary with group size, sex, season, activity engaged in prior to disturbance, previous exposure to noise source, and distance from noise source.

\author{
Presentation of the Silver Medal in Engineering Acoustics to Hugh S. Knowles
}

\author{
Presentation of the Silver Medal in Architectural Acoustics to Theodore J. Schultz
}




\title{
Session EE. Physical Acoustics V and Engineering Acoustics IV: Atmospheric Acoustics III
}

\author{
William L. Clink, Chairperson \\ Atmospheric Environmental Services, Downsview, Ontario M3H $5 T 4$ Canada
}

\section{Invited Papers}

\begin{abstract}
$8: 30$
EE1. A coustic sounding of the atmosphere after ten years. Freeman F. Hall, Jr. (Wave Propagation Laboratory, ERL/NOAA, Boulder, CO 80302)

It has now been ten years since the Weapons Research Establishment in Australia precipitated the modern era of atmospheric acoustic sounding with the operation of their $15-\mathrm{m}$ dishequipped sounder in November 1966 . The spectacular facsimile records of atmospheric structure resulting from this pioneering effort have caught the interest of a large segment of the atmospheric sciences community. The number of such sounders now operated around the world approaches 200 . From the outset, the utility of the devices for measuring the height of temperature inversions as the atmosphere passes through its diurnal cycles was recognized, and this ability is being exploited by many industrial and governmental groups concerned with air pollution problems. In addition, the ability to quantitatively measure the intensity of the scattered sound has led to a much improved understanding of the structure of atmosphere turbulence. Frequency analysis of the scattered signals allows accurate measurements of the three dimensional winds throughout the planetary boundary layer, and several prototype wind profiling systems are now in routine operation. The discipline still has many problems to be solved and questions to be answered: Can small scale turbulence measurements be extrapolated to predict pollutant diffusion? Can molecular and scattering attenuation coefficients be determined well enough to enable accurate measurements of turbulence throughout the planetary boundary layer? Can advantage be made of the coherence properties of the transmitted signal? Can temperature and humidity profiles be measured acoustically? Can acoustic Doppler wind sensors utilizing modern digital techniques be made inexpensive enough for urban networks to be established?
\end{abstract}

\section{Contributed Papers}

\section{9:00}

EE2. Comparison of Doppler sodar antenna configurations used for horizontal wind measurement. Robert $F$. Davey (A eroVironment Inc., 145 Vista Ave., Pasadena, CA 91107)

The performance of two antenna configurations is evaluated using a parametric optimization technique which predicts the relative accuracy of horizontal wind measurement. Consideration is given to the effect of atmospheric attenuation and the relation between the thermal and velocity structure functions as well as to the geometric effects. Using a baseline case with a maximum measuring altitude of $500 \mathrm{~m}$, optimum antenna orientations are determined for the familiar axisymmetric three-transmitter configuration and for a two-transmitter system using a tilted receiver antenna. The sensitivity of each system to small changes in the geometry and to modifications in the assumed attenuation and structure function ratio is examined. The two-transmitter system is found to generate velocity measurements with accuracy comparable to the three-transmitter geometry at the design point. However, the effect of the preferred orientation at lower heights results in poorer comparative performance.

\section{$9: 15$}

EE3. High-powered acoustic transmitter for atmospheric sounders. J. Tant Priestley (Wave Propagation Laboratory, NOAA Environmental Research Laboratories, Boulder, CO 80302)

A high-power narrow-beam acoustic transmitter for use in an atmospheric sounder has been designed and evaluated. The transmitter uses thirteen electroacoustic horn drivers manifolded to a $2-\mathrm{m}$ aperture horn-reflector antenna. The evaluation of the transmitter included measurements of beam pattern, total efficiency, efficiency of individual drivers, total power output, and finite amplitude distortion. In particular at a frequency of $1250 \mathrm{~Hz}$ the $3-\mathrm{dB}$ beamwidth was $9^{\circ}$, the total efficiency was $10 \%$, individual driver efficiency was approximately $17 \%$, and the total power output was approximately 50 acoustic watts. At this power power finite amplitude distortion was quite perceptible at the mouth of the horn.

$$
9: 30
$$

EE4. Three recent advances in acoustic sounding instrumentation and data handling at SRI. E. E. Uthe, P. B. Russell, and N.B. Nielsen (Atmospheric Sciences Laboratory, Stanford Research Institute, Menlo Park, CA 94025)

Three recent technology developments at SRI are being used to improve the quality of acoustic sounder measurements and to facilitate the storage and interpretation of acoustic data. These are (1) Dual digitization and television display of simultaneous acoustic sounder and lidar data using a minicomputer-based system [E. E. Uthe and R.J. Allen, Opt. Quant. Electron. 7, 121-129 (1975)]; (2) Substitution of a larger dish and enclosure for the standard Aerovi ronment model; and (3) Microfilm storage of acoustic records, with machine-assisted transfer of layer heights and transition times to computer cards or tape. Examples demonstrating the utility of each technique will be shown. 
$9: 45$

EE5. Influence of echo-carrier-frequencies and antennadimensions on the performance of echosonde antennas.

S. Adeniyi Adekola (NOAA/ERL/Wave Propagation Laboratory, $\mathrm{R} 45 \times 5$, Boulder, $\mathrm{CO} 80302$ )

Accurate computer-simulations of echosonde antennas can contribute toward (1) better understanding of acoustic remote sensing of atmospheric structure/properties and (2) timesaving and avoidance of unforeseen problems in fieldwork. However, computer-predicted patterns are at odds with measurements, particularly regarding phase variations across transmitting antenna apertures. The rapidly changing phase variations of previous computer simulations do not compare well with nearuniform phase of measurements. Modifications of antenna design which improve antenna-system performance, are considered. These are based on the results of a general antenna-integral-formulation [Adekola, J. Acoust. Soc. Am. $60,230-239$ (1976)l. By proper choices of echo-carrierfrequencies and antenna-dimensions, a quasiuniform phase distribution, in excellent agreement with modest phase shifts measured experimentally [Hall and Wescott, J. Acoust. Soc. Am. 56, 1376-1382 (1974)I, is obtained by computer-simulations. Results of computer-predicted patterns and comparisons between theory and recent pattern measurements [Wescott, private communications (1976)] are presented. A meusured 3-dB pattern beamwidth of $13^{\circ}$ (average of two measurements) compares well with computer-prediction of $12.8^{\circ}$. Severe sidelobes are major detriments in Acoustic Remote Sensing. Other results presented include (1) ground-level sidelobes versus echo-carrier-frequencies, (2) 3-dB beamwidth variations with echo-carrier frequencies and antenna dimensions, and (3) computer simulations of the antenna type employed in probing marine-atmosphere remolely from a moring ship. [Mandies and Owens, J. Appl. Meteorol. 14, 1110-1117 (1975)] during a cruise in the Pacific Ocean/ Caribbean Sea. IWork supported by NRC.I

\section{0:00}

EE6. Digitized acoustic radar signals. Ch. Werner (Institute of Atmospheric Physics (DFVLR), Oberpfaffenhofen, Federal Republic of Germany D-8031)

To study the atmospheric structure, a data acquisition system for the monostatic acoustic radar was developed. It allows the direct comparison to in situ and other remote measurements. The data acquisition system consists of a transient recorder, an interface and a magnetic tape recorder. The transient recorder digitizes and stores the signal. The interface, containing an adjustable calender and a real time digital clock is connected between the transient recorder and the magnetic tape recorder. The tape recorder writes data in an IBM compartible mode. A special computer program to get lines of the same signal amplitude is used. There is more detailed information as the normal display unit shows. Examples demonstrating the possibilities of this digitized system are given.

\section{0:15}

EE7. Use of a nearfield array for acoustic backscattering cross-section measurements. Ralph Fiorito and Walter Madigosky (Naval Surface Weapons Center, White Oak Laboratory, Silver Spring, MD 20910)

It is theoretically demonstrated that a large circular piston in an infinite rigid baffle operating at high frequency can be used as a nearfield array (NFA) to measure farfield backscattering (sonar) cross sections. Pulsed experiments are described which use the same piston both to insonify the object and receive the scattered wave. In transmitting, the piston produces a collimated plane wave within its Rayleigh zone. When the scatterer is placed within this zone, the scattered wave is received by the piston and integrated to reproduce a voltage response proportional to the scatterer's farfield amplitude function (NFA reciprocity principle). Measurements have been made on spheres and cylinders as well as model ships, insonified by a 4.75-in. circular bearn at a frequency of 15 MHz. The sonar cross sections (target strengths) obtained are in good agreement with those thec retically predicted by finite beam insonification scattering theory. Comparison of scaled model ship measurements with full-scale target strength values shows reasonable agreement as well. [Work supported by NAVSEA-034. I

\section{0:30-BREAK}

$10: 45$

EE8. Doppler velocity extraction from atmospheric acoustic echoes using a level crossing technique. H. Melling and R. List (Department of Physics, University of Toronto, Toronto, Canada, M5S 1A7)

The scattering of a narrow-band acoustic signal from atmospheric turbulence generates an echo of rapidly varying amplitude and frequency. The mean frequency of the echo power spectrum is related by a Doppler shift to the mean motion of the scatterers. In the level crossing technique this shift is deduced from measurements of the "instantameous" envelope amplitude and rate of zero crossing of one component of the complex echo waveform. Data interpretation is based on an analysis of the statistics of instantane.sus echo frequency and power which shows the probability density function of frequency to depend only on the first two momenis of the power spectrum [R.C. Srivastava and R. E. Carbone, Radio Sci. 4, 381-393 (1969)]. These moments may be determined from averages in the time domain without the need for Fourier transformation. The technique has been tested on an electronically simulated echo signal and found to yield statistics in good agreement with the theoretical predictions. Similar tests on atmospheric echoes shou' good agreement for the frequency statistics but not for those of power.

$11: 00$

EE9. Some zero-crossing statistics of a signal consisting of band-limited white Gaussian noise with a pure tone added to it. R.O. Rowlands (Applied Research Laboratory, The Pennsylvania State University, P.O. Box 30, State College, PA 16801)

The number of zero crossings per second of a band-limited white Gaussian noise signal $x(t)$ is slightly greater than twice the midband frequency of the signal. This may he explained by considering the complex signal $z(t)=\hat{x}(t)+j x(t)$ where $\hat{x}(t)$ is the Hilbert transform of $x(t)$. The vector $z(t)$ rotates at a mean angular frequency that is equal to its mid-band angular frequency, but it has a jitter superimposed upon it that; on occasion, causes it to rotate in the opposite direction. The extra crossings are produced by the vector rotating backwards just after it has crossed the real axis in the forward direction. It is the statistics of these backward crossings that have been studied when a pure signal has been added to the noise. [Work supported by ONR Code 436.

\section{$11: 15$.}

EE10. A coustic noise cancellation techniques. R. Jeffrey Keeler (National Oceanic and Atmospheric Administration, Wave Propagation Laboratory, Boulder, CO 80302)

The environment noise that plagues dcoustic echo sounder systems is described as a nonstationary, non-white and nonisotropic random process. Classical detection and estimation theory do not treat this type of noise and consequently ad hoc procedures have been developed which will reduce the detrimental effects of acoustic noise. For simple monostatic echosondes the noise limits the dynamic range of recelved echoes by masking low-level signals and for Loppler echosondes the noise generates spikes in the received power spectrum which cause the variance of the resulting wind estimates to be increased. Several alternative schemes are given for reducing the effect of noise in the spectrum of such power-spectrumbased Doppler processors. Results from the implementation of some of these methods are shown. A time domain adaptive 
noise cancellation $(\mathrm{ANC})$ technique is described which potentially of fers a 5-20-dB improvement in SNR over any power spectrum noise subtraction scheme. The ANC method uses an external reference noise to estimate the noise on the signal which is then subtracted from the primary signal input. Thus the reference noise that is correlated with the primary input noise is effectively cancelled. Being a time domain preprocessor ANC can be employed on any echosonde.

$$
11: 30
$$

EE11. Preprocessors for real-time spectrum analysis. M. Andrews (Department of Electrical Engineering, Colorado State University, Ft. Collins, CO 80523)

An adaptive nonrecursive (NR) filter with application to spectrum analysis is presented. The adaptive nature is implemented with a weight adjustment algorithm (LMS) on the filter that performs a steepest descent minimization of mean-square error. The difference between an "interference" or coherent representative of it and the raw signal under analysis via its spectral content is defined as the error. A priori knowledge of the disturbances consists of the spectral content of the disturbances without regard to amplitude or phase information. The algorithm is manifest with a digital arithmetic unit using high-speed logic in a robust $\mathbf{5 1 2}$ weight digital filter fabricated with current integrated circuit technology. The experimental configuration is described along with improvement s currently attainable with advancements in technology. Applications to spectrum analysis, whereby unknown disturbances degrade interpretation of spectrum signatures in bearing studies, acoustic phenomena, and sonar signal analysis, is described. Adaptive filter behavior with stationary as well as nonstationary input signals are presented. It is shown that for some narrowband as well as broadband disturbances the filter tracks and adaptively rejects disturbance in real time so as to present uncluttered renditions of the spectrum signatures or spectograms for analysis.

\section{$11: 45$}

EE12. Sonar velocity resolution with a linear period modulated pulse. Richard A. Altes (Science Applications, Inc., P. O. Box 2351, La Jolla, CA 92037)

For linear period modulated (LPM) signals, the phase of the echo from a moving point target contains information about range rate. When an LPM signal is transmitted, a Doppler insensitive sonar processor is obtained by following a matched filter with an envelope detector. Alternatively, the same echo can be used for accurate velocity measurements by implementing a phase-sensitive detector. A receiver that envelope detects matched filter responses and also estimates the phase of an echo can therefore be both Doppler tolerant and Doppler resolvent. The relative simplicity of Doppler tolerant receivers is again realized when Doppler-sensitive processors are implemented for LPM echoes; if LPM is used, only two matched filters are needed to implement a sequence of $M$ velocity hypotheses, rather than a bank of $M$ different filters. LPM is thus an excellent signal format not only for Doppler invariant range estimation and initial detection, but also for fine velocity resolution with minimum receiver complexity. These observations may imply some hitherto unsuspected echolocation capabilities for bats that use LPM waveforms. The existence of velocity induced phase transformations in LPM signals also leads to a new synthetic aperture sonar technique. Iwork supported by Naval Coastal Systems Laboratory. 1

\title{
Session FF. Underwater Acoustics V: Impulse Sources
}

\author{
Peter Rogers, Chairperson \\ Naval Research Laboratory, Orlando, Florida 32086
}

\section{Invited Papers}

$8: 30$

FF1. Survey of nonexplosive sources for acoustic propagation measurements. J.H. Cawley (Arthur D. Little, Inc., Cambridge, MA 02140)

Since the mid 1940's explosive charges have been widely used by off shore seismic exploration companies, by the U.S. Navy, and by other groups to generate high-intensity acoustic pulses for seismic and sonar-related activities. In the late 1950's offshore exploration groups began to develop alternate energy sources, and in the mid 1960's a number of nonexplosive impulse and cw marine sound sources were introduced and soon dominated the offshore exploration field. Despite their relatively low-energy levels, these sources have since been tailored to reprodueibly generate acoustic spectra concentrated in the cange below $100 \mathrm{~Hz}$ at efficiency levels that have allowed them to completely replace shallow-detonated explosives. This report briefly describes important characteristics of four classes of nonexplosive acoustic impulse sources developed for offshore seismic exploration purposes and compares their acoustic signatures with those of explosive SUS charges used in sonar propagation studies. The total energy levels of SUS and several nonexplosive impulse sources are compared and acoustic levels, bubble pulse, and the spectral characteristics of the signatures are discussed and methods for defining and controlling these characteristics are briefly deseribed. [Work supported by ONR/LRAPP.] 
FF2. Hydroacoustic impulse sound source. J. I. Bouyoucos (Hydroacoustics Inc., P.O. Box 3818, Rochester, NY 14610)

An impulse source concept is described in which the potential energy of the pressure head of an underwater environment is converted to kinetic energy of an imploding flow which, in turn, is converted to an acoustic impulse free of after-shot clutter. This energy conversion concept has been realized in various hardware forms under the designation HYDROSHCrK. (\$) It is characteristic of this class of source that considerable latitude exists in specifying the spectral level and spectral shape of the transmitted signal. The conversion mechanisms will be outlined, and the properties of several of the existing HYDROSHOCK ${ }^{\circledR}$ devices will be described. One recently developed source exhibits a peak source level of $255 \mathrm{~dB}$ re $1 \mu \mathrm{Pa}$ at $1 \mathrm{~m}$, a pulse duration of $40 \mu \mathrm{sec}$, and a useful spectrum extending from $20 \mathrm{~Hz}$ to $100 \mathrm{kfz}$. The trade off $s$ in source configuration between source level and bandwidth will be described, and comparisons will be made to spark sources, air guns, and various explosive devices. Applications for such impulse sources will also be reviewed. TThis research has been supported by the Office of Naval Research, Code 222.1

\section{9:20}

FF3. Airgun array design techniques. T. L. Teer (Geophysics Research Department, Shell Development Company, P.O. Box 481, Houston, TX 77001)

Since airguns were introduced to the oil exploration industry in the late 1960's, they have replaced dynamite as the primary acoustic source for marine seismic profiling. A rrays of airguns compare favorably in the seismic bandwidth to dynamite for depth of penetration and resolution. Multi-element arrays capable of peak-to-peak pressures exceeding 50 bars (at 1 meter) are presently being used in the industry. Independent control of chamber pressure and volume, operating depth and distance between airguns allows the design of airgun arrays which can meet particular resolution or depth of penetration objectives. Array design lechniques are reviewed and data from a Shell array is presented to illustrate the degree to which success in meeting the design objectives of a high peak pressure, a high primary-to-bubble ratio and broad bandwidth is achieved. Far-field signatures from the array are shown to possess the necessary shot-to-shot stability required of modern signal enhancement techniques when proper care is taken in deploying and towing the array. Boundary effects (ghosting) are shown to reduce shot stability. Some mechanical deployment and towing considerations are discussed.

$9: 45$

FF4. Underwater explosion sources. Ermine A. Christian (Naval Surface Weapons Center, White Oak, Silver Spring, MD 20910)

Explosions have been used as underwater acoustic sources for many years, sometimes at rates exceeding 50000 charges a year. During the past decade the types of studies undertaken with explosion sources have changed in character, and the requirements on charge levels have become more demanding. For example, an accuracy of $\pm 1 \mathrm{~dB}$ in narrow-band levels is now desired for propagation-loss measurements in the 25 to $300 \mathrm{~Hz}$ frequency range. Fixed explosion source levels of such accuracy can only be accomplished if the charge depth is closely controlled; but strict depth control severely limits the type of experiment that is feasible. Compromise techniques for effective utilization of explosion sources are being developed. Recent progress along two avenues of attack on the problem-improved methods of describing the explosion sound field, and of taking the source characteristics into account during farfield data analysis-will be reviewed.

10:10

FF5. A computer model of the explosive acoustic source. R. B. Lauer and L.C. Maples (Naval Underwater Systems Center, New London Laboratory, New London, CT 06320)

Explosives are widely used as broadband acoustic sources, but their spectrum characteristics are not adequately defined. A comprehensive experimental evaluation is not feasibie economically and the problem can be solved only by a model, supported by a limited critical experiment. A computer model of the source waveform, using only analytic functions, is at last available. The model, known as DIAL-A-SUS, readily provides the desired spectrum for any charge size and detonation depth by Fourier transform techniques. Certain amplitude and time characteristics of the waveform, which are defined in terms of the source we.ght and depth, are used to evaluate the parameters of the model's analytic functions. The model is ideally suited to study the sensitivity of the spectrum characteristics to variations in the critical parameters of the waveform and determine the important factors requiring furt her experimental evaluation. The results and significance of such studies are discussed, with experimental implications, and model predictions are compared with measurements. An exciting spinoff is the characterization of the explosive as a multifrequency, narrow-banc (e.g., $1 \mathrm{~Hz}$ ) source, with important potential applications. [Work supported by NUSC.] 


\section{Contributed Papers}

$10: 36$

FF6. Low-frequency underwater explsoive source levels. R. C. Hughes (Defence Research Establishment Atlantic, P.O. Box 1012, Dartmouth, Nova Scotia, Canada, B2Y 3Z7)

New experimental measurements of underwater source levels for explosive charges at depths ranging from 20 to $200 \mathrm{~m}$ are reported. The explosives used include $45 \mathrm{~g}(1.6 \mathrm{oz})$ TETRYL primer charges, $454 \mathrm{~g}(1.0 \mathrm{lb})$ bare TNT charges, $31 \mathrm{~g}(\mathbf{1 . 1}$ oz) TETRYL SUS charges and $816 \mathrm{~g}(1.8 \mathrm{lb})$ TNT SUS charges. Underwater pressure measurements were made at a nominal distance of $1500 \mathrm{~m}$. For frequencies below approximately twice the bubble frequency, the experimentally measured acoustic energy spectra show a remarkable correspondence when they are appropriately scaled for detonation depth and for charge weight. A universal model of the low frequency energy spectra of TNT and similar explosives, which can be scaled to different charge weights and different detonation depths, shows considerable promise.

\section{$10: 48$}

FF7. Source level measurements of $1.8 \mathrm{lb}$ and $1.1 \mathrm{oz}$ underwater sound signals. J.M. Thorleifson and P. D. Boyle (Defence Research Establishment Pacific, Forces Mail Office, Victoria, British Columbia, Canada, VOS 1BO)

The source levels of underwater sound signals (SUS), containing $1.8 \mathrm{lb}$ and $1.1 \mathrm{oz}$ of TNT detonated at 60 and $600 \mathrm{ft}$, were measured experimentally at Jervis Inlet in November 1974. The measurements were made by recording the source pressure-time waveforms received on hydrophones placed about $1000 \mathrm{ft}$ below the detonation depth. The measured source levels are compared with levels computed using a model based on Weston's analytical model [D. E. Weston, Proc. Phys. Soc. 76, 233-249 (1960)] and with Gaspin and Shuler's results [J.B. Gaspin and V.K. Shuler, Naval Ordnance Lab TR 71-160, Oct. 1971] for $1.8 \mathrm{lb}$ of TNT detonated at $60 \mathrm{ft}$. For the $1.8-\mathrm{lb}$ sources, the measured results are up to $5 \mathrm{~dB}$ different from the levels computed using the Weston model over the band $10-1600 \mathrm{~Hz}$. However, the measured levels are in good agreement with those of Gaspin and Shuler. For the 1.1-oz source the measured results are up to $20 \mathrm{~dB}$ lower than the levels computed using the Weston model. This large reduction in level is likely due to energy lost in breaking through the SUS container.

\section{$11: 00$}

FF8. Nearfield data from small and large explosions. I. Timedomain analysis. Jean A. Goertner and Ira M. Blatstein (Naval Surface Weapons Center, White Oak, Silver Spring, MD 20910)

In a joint sea test of the Naval Research Laboratory, the White Oak Laboratory has obtained nearfield explosion data from 8-, 48-, and 1000-lb TNT charges. Pressure-time measurements at constant range were made, with multigain recordings at two depths. Recordings were made using both a ceramic hydrophone and a tourmaline piezoelectric gage. The experiment will be described and experimental data presented. These shallow depth-constant range data validate previous results for underwater explosion similitude expressions for pressure, decay constant, etc., that were largely obtained from data where range and depth effects were intertwined. The data also extends to shallower depths the reduced parameter curves currently employed for predicting underwater explosion effects and for modeling acoustic source levels. Discussion will focus on how comparison of various records are useful for comparing different sensor outputs and for studying nonlinear effects-factors that influence source level determination. [This work was funded by DNA.]

11:12

FF9. Nearfield data from small and large explosions. II: Spectral analysis. Joel B. Gaspin and Jean A. Goertner (Naval Surface Weapons Center, White Oak, Silver Spring, MD 20910)

The problem of the definition of the spectral source energy levels for the joint NSWC-NRL experiment, discussed in the previous paper, will be treated. Since surface reflections occur in the pressure records, the removal of surface reflection effects is necessary in order to obtain the source spectra. The spectral modulation pattern due to the surface reflections are different at the two sensor depths. This makes these data well suited for the examination of different techniques for demodulating the spectrum. Both time and frequency domain deconvolution and various smoothing techniques are examined, and their effects on the spectral energy in $\frac{1}{3}$-octave bands are demonstrated. Certain computational difficulties arising in the frequency-domain deconvolution are avoided by use of timedomain deconvolution. In situations where the surface reflection is not acceptably coherent, or if cavitation occurs, simple deconvolution is not applicable, and special techniques must be used. The dependence of the source spectra on sensor type is also examined. The source energy levels derived from the $1000-\mathrm{lb}$-charge data are found in good agreement with the scaled-octave band source level curves of Christian [J. A coust. Soc. Am. 42, 905(L) (1967)], which were derived from much smaller charges. [This work was funded by DNA.]

$$
11: 24
$$

FF10. Weak-shock solution for explosive shock waves. Peter H. Rogers (Naval Research Laboratory, Underwater Sound Reference Division, P.O. Box 8337, Orlando, FL 32806)

The initial pressure wave measured at modest distances from an underwater explosion is often modeled as a spherical shock wave with an exponential decay. A closed-form analytical "weak-shock" solution for the subsequent propagation of such a wave has been obtained. Simple formulas expressing the peak pressure and initial decay constant as function of reduced range are presented. These allow the prediction of the amplitude and initial slope of the wave given only the amplitude and decay constant of the original exponential shock and the density, sound speed and parameter of nonlinearity of the water. The results are in good agreement with the KirkwoodBethe theory, measurements, and the widely used experimentally based semiempirical similarity formulas. An expression which gives a close approximation to the waveform as a function of distance is also derived. 


\title{
Session GG. Shock and Vibration IV
}

\author{
Clive Dym, Chairperson \\ Bolt Beranek and Newman, Inc., Cambridge, Massachusetts 02138
}

\section{Contributed Papers}

$8: 30$

GGl. Antisymmetric modes of vibration of a circular plate elastically restrained against rotation and subjected to a hydrostatic state of in-plane stress. P. A. A. Laura, L. E. Luisoni, and A. Arias (Institute of Applied Mechanics, Base Naval, Puerto Belgrano, A rgentina)

The analysis of flexural vibrations of plates with edges elastically restrained against rotation is of interest to the design engineer since ideal supports or clamps are difficult to obtain in practice. A survey of the literature reveals that several investigations have been performed in the past on (a) vibrating circular edges (without in-plane loading) and (b) vibrating simply supported and clamped circular plates subjected to hydrostatic in-plane loading. The present paper deals with the determination of very simple, approximate frequency equations which allow prediction of natural frequencies in the case of a vibrating circular plate which executes antisymmetric modes. It is shown that use of simple polynomial expression and a variational approach leads, in some cases, to numerical values which are more accurate than those available in the technical literature. The approach followed in the present investigation can be extended in a straightforward fashion to the case where the edge has translational flexibility.

\section{8:45}

GG2. Vibrations of prolate spheroidal shells of constant thickness. Courtney B. Burroughs (Bolt Beranek and Newman Inc., Cambridge, MA 02138) and Edward B. Magrab (Institute for Basic Standards, National Bureau of Standards, Washington, DC 20234)

The general displacement-equilibrium equations, which include the effects of transverse shear and rotary inertia, have been derived for a prolate spheroidal shell of constant thickness. The solution is formulated for a shell that is immersed in an inviscid fluid of infinite extent and subjected to an harmonically time-varying, arbitrarily spatially distributed force normal to the shell surface. The approximate formal solutions for the three displacements of the shell surface and the two rotations of the shell cross section are obtained using an extension of Galerkin's variational method developed by Chi and Magrab [Proceedings of the International Conference on Variational Methods in Engineering (University of Southampton, 1974)]. Numerical results are presented for the lowest seven axisymmetric natural frequencies of the shell in vacuo. Using 15-term solutions for both thick and thin shells, which have eceentricities that vary from 0.46 to 0.99 , the approximate natural frequencies are found to converge to within less than $1 \%$ their final value. Good agreement with other published results for the approximate natural frequencies of a thin prolate spheroidal shell and for the exact natural frequencies of a thick spherical shell is obtained. Additional results for the natural frequencies of moderately thick shells as a function of shell eccentricity, mode number, and shell thickness are presented.

$$
\text { 9:00 }
$$

GG3. Flexural waves generated in glass fibers by fracture. P. G. Simpkins (Bell Laboratories, Murray HIll, NJ 07974)

In 1948 Davies noted that flexural waves could be created in a rod by an unsymmetrical load applied to one end. Miklowitz applied this observation to the hypothe'sis that during tensile fracture a moment pulse is exerted or, the specimen which generates a flexural wave. He went or to suggest that this flexural wave could augment the stress induced in the rod by a reflected longitudinal wave when the two were coincident; there-by causing the secondary failures observed in brittle materials. The nature of the failure in fused silica fibers qualitatively suggests that it alters as the st::ength increases. While the low-strength fibers simply fracture at one point, the high strength fibers (UTS $>2.8 \mathrm{GN} / \mathrm{m}^{2}$ ) apprear to disintegrate into a particulate cloud. Recent photograplic studies show that a flexural wave emanates from the primary fracture. When the strength is sufficient this flexural wave causes secondary explosive failures at other points along the fiber. These result in a particulate cloud of debris as the fiker appears to be progressively pulverized. Observations of the flexural wave amplitude show it to be a strong function of fiber strength. The observed group velocity of the flexural waves compares favorably with the classical time harmonic theories and the first-mode Timoshenko approximation.

9:15

GG4. Time-average holographic study of a singing wine glass. R. Tonin and D. A. Bies (Department of Mechanical Engineering, University of Adelaide, Adelaide, South Australia 5001)

Previous attempts to determine the motion of a free edge vibrating cylinder using time average optical holography have been inconclusive. On the assumption of strictly radial motion attempts at the interpretation of the fringes produced in orthogonal views has lead to the contradiction that the implied motion motion was not single valued IS. D. Lism et al., J. Sound Vib. 29, 475-481 (1973)]. In the latter reference it was suggested that the problem lay with the physical location of the optical fringes in the reconstructed image. However, as already shown by Rayleigh the motion of the free edge of a vibrating cylinder has both radial and tangential components of displacement and this point had not been laken into account. In this paper it is shown how the theory of time-average optical holography may be extended to account for generalized elliptic motion of a vibrating surface under study and how the optical system may be arranged to unambiguously interpret the motion. It is shown that good argument is obtained between the predicted and observed motion for a singing wine glass and for a free edge rectangular cylinder vibrating in a Love mode.

GG5. Vibrations of two concentric cylindrical shells containing a viscous fluid. T.T. Yeh and S.S. Chen (Components Technology Division, Argonne National Laboratory, A rgonne, IL 60439)

This study was motivated by the need to design the thermal shield in reactor internals to avoid detrimental flow-induced vibrations. The system component is modeled as two coaxial shells separated by a viscous fluid. In the analysis, Flugge's shell equations of motion and linearized Navier-Stokes equa- tion for viscous fluid are employed. F.rst, a traveling-wavetype solution is taken for shells and fll id. Then, from the interface conditions between the shells and fluid, the solution for the fluid medium is expressed in terms of shell displace- 
ments. Finally, using the shell equations of motion given the frequency equation, from which the natural frequency mode shape, and modal damping ratio of coupled modes can be calculated. Some important conclusions are as follows: (1) There exist structural modes and acoustic modes. If the structural natural frequencies and mode shapes are of primary interest, the fluid may be considered inviseid and incompressible. (2) There exist out-of-phase and in-phase modes. The lowest natural frequency is always associated with the out-of-phase mode. (3) The lowest natural frequeney of coupled modes are lower than the uncoupled modes. (4) The fluid viscosity contributes significantly to the modal damping.

9:45

GG6. On the forced responses of damped cylindrical shells filled with pressurized liquid. Y.P. Lu (David W. Taylor Naval Ship Research and Development Center, Annapolis, MD 21402)

An analytical formulation for the forced vibratory responses of a pressurized liquid-filled cylindrical shell having a number of mass segments adhered to it by a viscoelastic material is presented. The mass segments are discretely distributed around the outer circumference at an arbitrary section of the shell, and the excitation is a concentrated vibratory radial force located on the surface of the shell. The end conditions of the shell are assumed simply supported. The liquid is considered as a compressible and inviscid fluid. The viscoelastic material is assumed incompressible. The interaction between the shell and the attached mass segments, and the interaction between the shell and the enclosed liquid are taken into consideration. The driving point mechanical impedances at a location midway between two mass segments and half the distance along the length for a given damped system with or without pressurized water are given. These solutions are compared and discussed, respectively, with those of an undamped shell. The responses of a discontinuously constrained damped ring configuration without liquid enclosed and the frequencies of an undamped shell with pressurized water filled, which are the special cases derived from the analysis presented, are, respectively, compared very well with the available experimental data.

\section{0:00}

GG7. Loss and coupling loss factors of two coupled dynamic sy stems. G. Maidanik and J. E. Brooks (David W. Taylor Naval Ship Research and Development Center, Bethesda, MD 20084)

The Statistical Energy Analysis (SEA) has been employed to define and estimate the response of complex dynamic systems. Often a complex dynamic system is conveniently divided into a number of subsystem-basic dynamic systems. The complex is then modeled in terms of a number of coupled (basic) dynamic systems. Within the format of SEA the deseritpion of the model is given in terms of the loss and coupling loss factors of the dynamic systems that compose the complex. Thus, to define the model, means must be devised to obtain the values of the Ioss and coupling loss factors. In this paper the experimental methods for obtaining these values are considered and scrutinized. For the most part considerations are limited to a complex consisting of two dynamic systems; the elements of an extension to higher forms of complexes are, however, included. It is shown that to obtain experimentally the loss and coupling loss factors one is required to conduct not only steady state but also transient experiments. The role of the reverberation time determined in the transient experiments is of particular interest.

\section{$10: 15$}

GG8. Use of the complex dynamic shear stiffness of a viscoelastic liquid to illustrate the origins of structural damping. R. A. Ely (Vought Corp., P.O. Box 5907, Dallas, TX 75222)

The same complex stiffness notation which causes damping of solids and structures to appear to be independent of fre- quency and dependent on amplitude is shown to have descriptions of frequency-dependent, velocity-dependent, viscous damping hidden within it. That revelation is consistent with recent papers which report that only a viscous component of structural damping need be measured or modelled mathematically. Those papers also report that viscous damping accounts for all of the energy loss from built-up structures (as elementary dimensional analysis shows it must). This paper draws upon measured values of the dissipative and elastic components of the complex dynamic viscosity and stiffness of a viscoelastic liquid to illustrate conceptual continuity from damping in liquids to damping in solids.

\section{0:30}

GG9. Response of nonclassically damped systems to random excitation. C. D. Michalopoulos (Department of Mechanical Engineering, University of Houston, Houston, TX 77004)

The response to stationary random excitation of multidegreeof-freedom sy stems which do not possess classical normal modes is obtained using a matrix perturbation technique. It is assumed that the damping matrix $\mid C l$ is such that the offdiagonal elements of the matrix $\left.[\Phi]^{T}[C] \mid \Phi\right]$, where $[\Phi]$ is the modal matrix associated with the undamped system, are small compared to its diagonal elements. General expressions for the correlation and spectral density matrices of the response are given. Examples of systems with two degrees of freedom are presented considering first and second orders of perturbation.

10:45

GG10. Identification of natural frequencies and modal damping ratios from response data. C. D. Michalopoulos (Department of Mechanical Engineering, University of Houston, Houston, TX 77004)

Response data are processed in the frequency domain to determine natural frequencies and modal damping ratios of a multidegree-of-freedom system. The excitation can be either deterministic or a random function of time. When the time history of the excitation is now known, the method requires its power spectral density to be flat. An iterative scheme is employed to minimize a mean-square error function between the theoretical and measured power spectral densities of the response. In processing random response data, special steps are taken to minimize the statistical error. (Work supported by NASA.I

$11: 00$

GG11. Errors in power spectral density estimates of vibration test data. M.E. Austin (Department of Electrical Engineering, University of Texas, El Paso, TX 79968)

Estimates of power spectral densities obtained using timecompression, swept-filter spectral analyzers may contain errors due to assumptions regarding normality, randomness and symmetry of the data being processed. This paper considers possible errors in these estimates caused by various effects. The first concern is for hidden periodic components in data records assumed to be entirely random. Expressions describing this error are developed. Also of concern are nonlinear effects in the instrumentation which may result in amplitude-limiting of data records. Error analysis is performed for several possible amplitude distributions. Finally errors due to asymmetrical data records are considered. Records which are not normally-distributed in amplitude exhibit some degree of asymmetry and thus the power spectral density estimates obtained from these records will be in error. Expressions for power spectral density estimates of amplitude distributions other than normal are developed. These are compared with estimates obtained under the normal assumption. Also, general relationships regarding power spectral density estimates as a function of the skewness and kurtosis of the data record are discussed. 
$11: 15$

GG12. Analytical simulation and experimental measurement of stress wave propagation in guided projectile structures. D. M. Anderson, J. G. Hanse, and T. N. Helvig (Honeywell, Inc., Government and Aeronautical Products Division, 2600 Ridgway Rd., MN17-1612, Minneapolis, MN 55112)

An analytical simulation and experimental measurement of stress wave propagation through a guided projectile structure was made and the results correlated through the use of fast Fourier transform (FFT) methods. Two guided projectile structures were simulated; one with and one without bolted joints such that signal attenuation across a joint could be evaluated. The analytical simulation consisted of a linearly elastic finite element structural model subjected to a timehistory force input and solved using SAP IV via direct integration of the coupled differential equations. The experimental measurements were made using a pulse-testing technique where in a structure was instrumented with accelerometers and impacted $w$ ith a force input and the accelerometer response and force input measured. The resulting time history response data, whether analytical or experimental, was then analyzed using FFT methods to generate a frequency domain representation of the response signal for comparison. Results indicate finite element structural simulations of elastic stress wave propagation are a valid design tool for studying the noise characteristics of a structure being impacted with low order force inputs. [Work sponsored by Honeywell Independent Development Funding. ]

$$
11: 30
$$

GG13. Synthesis methods for an antivibration filter that is required to support the source mass. C.B. Putnam and R.O.
Rowlands (Applied Research Laboratory, The Pennsylvania State University, P.O. Box. 30, State College, PA 16801)

Classical network synthesis theory, that was developed for the design of electrical filters terminated in a resistance, may be applied to the design of anti-vibration mechanical filters, except when the filter has to support the source mass. A modified design method has been developed to procuce a termination consisting of a damped spring. Two ceses are considered: (1) when the mass of the source is small, it is incorporated in the design as the first reactive arm of the filter, and (2) when the mass is large, the design is first performed assuming the source mass to be the infinite and then modified to take into account the finite value of the mass. [Work supported by Naval Sea Systems Command.]

\section{1:45}

GG14. Preliminary analysis of tracked vehicle dynamics. A. G. Galaitsis, P.J. Remington, and T. Norris (Bolt Beranek and Newman Inc., Cambridge, MA 021.38)

A simplified model has been adopted to describe the nonlinear track/sprocket and track/idler interaction of a tracked vehicle. The differential equations of motion were obtained through the Lagrangian formulation and the time history of displacements and forces were computed by finite difference techniques. The predicted forces and measured force-to-noise transfer functions were, subsequently, used to project the interior vehicle noise which compares favorably to date. [Work supported by U.S. Army Contract DAAD005-76-C0748.]

\title{
Session HH. Speech Communication V: Synthesis and Vocal Tract Modeling
}

\author{
Cecil Coker, Chairperson \\ Bell Laboratories, Murray Hill, New Jersey 07974
}

\section{Contributed Papers}

9:00

HH1. Synthesis of English monosyllables by demisyllable concatenation. J.B. Lovins and O. Fujimura (Bell Laboratories, Murray Hill, NJ 07974)

The use of demisyllables plus voice-assimilated phonetic affixes $(/-s,-z,-t /$, etc.), rather than segments or syllables, as the basic units in speech synthesis would have important advantages lO. Fujimura, J. Acoust. Soc. Am. 59, S55(A) (1976)]. We have tested the practicality of such a model for monosyllabic words of English, using an inventory of demisyllables with a variety of nonaffixed clusters and syllable nuclei. Each initial demisyllable is prepared so as to include a constant length of the transitional portion, and nucleus length variations dependent on the final consonant(s) are automatically included in the "vowel" portion of the final demisyllable. Syllable nucleus "coloration" by postvocalic nasals, $/ r /$, or $/ l /$ is also found to be automatically accounted for in this scheme. For example, the initial demisyllable exeised from CVC may be joined with - $V N$ to make a natural-sounding CVN syllable. These experiments used both a parallel-type formant analyzer-synthesizer (J. P. Olive and M.J. Macchi, J. Acoust. Soc. Am. 58, S23(A) (1975)I and an LPC processor, with some retouching, and with minimal smoothing across the concatenative boundary of the parameters.

\section{$9: 10$}

HH2. Syllable synthesis. Ignatius G. Mattingly (Haskins Laboratories, 270 Crown St. , New Haven, CT 06510, and Department of Linguistics, University of Connecticut, Storrs, CT 06268)

In an attempt to provide a more direct representation of coarticulatory effects on spectral and temporal patterns of speech than is possible with a segmentally organized algorithm for synthesis by rule, an algorithm now being developed is organized around the syllable. The input is a phonetic deseription of the utterance in terms of syllable features and a set of rules. The rules determine the translation of the feature description into a description in terms of articulatory influences, such as those of the current vowel, the following vowel, initial and final semivowels, inilial and final con- 
sonants. Each such influence is represented by a set of target values for formant frequencies and by an exponential function of time; and the targets, the rates of growth and decay of the influence functions, and their timing relative to one another, are specified in the rules. The influences are ordered, and a formant-frequency value at a given time is determined by iteratively computing the sum of the target value associated with the $n$th influence, weighted according to its influence function, and the value determined by the first $n-1$ influences. This algorithm is a part of a synthesis by rule program written in FORTRAN for the PDP11/45. (Work supported by NIH and VA. I

\section{9:20}

HH3. English intonation and computerized sound synthesis. A rvin Levine (Department of Linguistics and Institute for Mathematical Studies in the Social Sciences, Stanford University, Stanford CA 94305)

Sentential intonation has been approached through the paradigms of acoustical engineering and linguistics. This research stresses the latter, treating pitch in English intonation as similar to tone languages [W. Leben, Linguistic Analysis 2.1 (1976)], and to song [M. Liberman, MIT dissertation (1975)]. Declarative intonation results from underly ing melodies, consisting of a few discrete tones, interacting with a sentence's syntax and semantics. The melody for declaratives is $\mathrm{M}-\mathrm{H}-\mathrm{L}$ (a midtone followed by high and low tones). The syntactic structure determines how this melody is elaborated to fit the sentence. Typical questions have a different underlying melody, M-L-H, but the syntactic control of elaboration is the same. The semantics alters the elaboration and not the choice of an underlying melody so that changing the focus of a sentence alters the elaboration of the underlying melody and thus the perceived stress. We make concrete proposals for automatic generation of intonation contours: adding to written text the necessary pitch, duration and loudness parameters for computerized sound synthesis. Our intonation procedure has been incorporated in a computer-aided instruction program.

$$
\text { 9:30 }
$$

HH4. Rule-generated pitch contour algorithm based on Junction Grammar linguistic model. R. Millett, A Melby, W.J. Strong. and E. G. Lytle (Physics Department, Brigham Young University, Provo, UT 84602)

A computer algorithm to generate pitch contours for various readings of an utterance is described. A Junction Grammar model of language containing semantico-syntactic information represented in "junction trees" is used as the basis for the algorithm. Recent developments of this model [Lytle, "Junction Theory as a Base for Dynamic Phonological Representation," Reported of BYU Linguistics Symposium (Provo, UT, 1976)] have provided a means for going from "junction trees" to "articulation trees" which contain language-specific segmental and prosodic information. The se "articulation trees" were used to generate a pitch contour for various readings of a sentence using one set of LPC analysis data. From a varied corpus of 10 sentences ( $10 \mathrm{LPC}$ sets), 23 different readings were synthesized using the algorithm to generate the pitch (fundamental frequency) contour in each case. Current research shows favorable preliminary results in expanding the algorithm to also predict pause and duration parameters in a word concatenation speech synthesis system.

\section{$9: 40$}

HH5. Evaluation of rule-generated pitch contours, A. Melby, R. Millett, W.J. Strong, and E. G. Lytle (Physics Department, Brigham Young University, Provo, UT 84602)

Research in modifying pitch contours in order to cause various readings to be perceived for a sentence (reported on at the November 1975 Meeting of the Society) has led to a com.puter algorithm for generating pitch contours from a semantico-syntactic representation of a sentence. These rule- generated pitch contours were evaluated according to two criteria: naturalness of intonation and "intelligibility" of intonation. Subjects were asked to rate sentences for naturalness on a scale and further to select the "natural" sentences from pairs of sentences. In a test of "prosodic intelligibility" subjects were asked to select which version of a sentence they heard (where stress was indicated by underlining or where various possible contexts were suggested). Stimuli consisted of arrangements of 35 distinct sentence versions synthesized from 12 sets of LPC analysis data, vary ing only the pitch (fundamental frequency) specifications. Included in the stimuli were versions with natural, rule-generated, manually specified, and monotone contours. Composite naturalness scores from part of the test placed rule-generated contours at 3.6 and the natural contours at 4.1 on a scale from 1 to 5 . Further results will be presented, as well as a discussion of how the results suggest specific areas of further research.

\section{9:50}

HH6. Fundamental frequency by rule for a text-to-speech system. Douglas O'Shaughnessy (Research Laboratory of Electronies, Massachusetts Institute of Technology, Cambridge, MA 02139)

An algorithm for the generation of fundamental frequency $\left(F_{0}\right)$ in a speech synthesis-by-rule system is presented. It accepts as input: syntactic information (part of speech of each word, and a surface tree representing the syntactic structure of the sentence) and lexical information (number of syllables and location of the lexically stressed syllable in each word, and simple phonemic information, such as: vowel versus obstruent versus sonorant, unvoiced versus voiced). In the overall system, such information is provided by parsing, lexical decomposition, and letter-to-sound rules. The output of the $\boldsymbol{F}_{0}$ algorithm is a sequence of $F_{0}$ values, one every $10 \mathrm{msec}$, used in synthesizing the voiced portions of the output speech. The algorithm makes use of research findings of the relationship of $F_{0}$ movements to the linguistic content of sentences. Such factors as stress, syntactic phrases, and sentence-type have direct $\boldsymbol{F}_{0}$ results: parts of speech and syntactic boundaries affect the degree and direction of $F_{0}$ movements, respectively, and questions have rising terminal $F_{0}$ and high $F_{0}$ throughout.

10:00

HH7. Structure of a complete text-to-speech system. Jonathan Allen (Research Laboratory of Electronics, and Department of Electrical Engineering and Computer Science, Massachusetts Institute of Technology, Cambridge, MA 02139)

The structure of a complete text-to-speech system, including lexical lookup, morphological analysis, parts-of-speech determination, parsing, letter-to-sound rules, lexical and phrase-level stress, morphophonemic rules, pitch and timing rules, and phonemic synthesis is presented. The interrelation of these processes is stressed, and the current status of the algorithm is described in relation to the quality of the speech output. Practical implementations (e.g. , in computer terminals, or reading machines for the blind) are also presented, including special purpose hardware. The design of these specialized devices is shown to be related to the structure of the algorithms to be implemented.

HH8. Use of LPC synthesized speech in an instructional application. W. R. Sanders and R. Laddaga (Institute for Mathematical Studies in the Social Sciences, Ventura Hall, Stanford University, CA 94305)

Synthesized speech was recently introduced into Stanford University's "Introduction to Logic" course. For five years, the course has been taught entirely by computer using teletypes to individually present all lesson material and on which all exercises are worked. Last spring, 28 to 70 students took $30 \%$ of the course on CRT-display terminals with earphones. 
They heard recorded messages encoded using autocorrelation LPC techniques and synthesized by a computer built at the Institute. The computer has 48 output channels and can speak independently on 16 channels at a time [Sanders, Benbassat, and Smith, "Speech synthesis for computer assisted instruction: the MISS system and its applications," ACM SIGGUE Topics 2, 200-211 (1976)]. The results of a questionaire indicated that more than two thirds of the audio students preferred the audio version. All of them felt the sound was clear; most preferred the speech presented faster than the original recording. Examples of the audio messages will be presented. [Supported by NSF Grant EPP-74-15016.]

\section{0:20}

HH9. How to put one person's tongue inside another person's mouth. Peter Ladefoged (Phonetics Laboratory, Linguistics Department, University of California, Los Angeles, CA 90024)

An algorithm has been devided for recovering the shapes of the tongue involved in producing different vowels simply from a knowledge of the first three formant frequencies. The calculated tongue shapes may then be displayed inside a standard vocal tract shape. The algorithm has been tested by recovering the tongue shapes of a wide variety of vowels spoken by a number of different speakers and comparing them with the original $x$ rays. The exact shapes of low vowels, such as $\{a, \alpha]$ are not recovered as accurately as those of other vowels, but the reconstructed tongue shapes of most men can be fitted very appropriately into a standardized diagram of the organs of speech based on $x$ rays of the author (Peter Ladefoged). There is inevitably some distortion of the organs when a woman's tongue is put inside the author's mouth, because of the notable anatomical differences betw'een men and women. The algorithm gives good approximations even when applied to speakers of other languages, such a Russian, Japanese, and Ngwe.

\section{0:30}

HH10. Determination of articulatory parameters of the human vocal traet from acoustic measurements. W.J. Möller (Drittes Physikalisches Institute, Universität Göttingen, Federal Republic of Germany, and Bell Laboratories, Murray Hill, NJ 07974), B.S. Atal (Bell Laboratories, Murray Hill, NJ 07974), and M.R. Schroeder (Drittes Physikalisches Institut, Universität Göttingen, F. R. Germany, and Bell Laboratories, Murray Hill, NJ 07974)

Previous work [B.S. Atal, J. Acoust. Soc. Am. 55, 384(A) (1974)] established that the articulatory parameters of a vocal tract model can be calculated from appropriate parameters of its acoustic output (such as linear predictor coefficients). The method is based on a "nonlinear" regression analysis involving both the acoustic parameters and their products. The weighting coefficients for the regression were determined in a "training session" in which the vocal tract assumed, sucessively, several thousand different configurations. The method is now being applied to human speech using $x$-ray data obtained by Fujimura, Miller, and Kiritani [J. Acoust. Soc. Am. 58, 540 (A) (1975)]. Preliminary results on estimating the height of the velum will be reported.

\section{$10: 40$}

HH11. Computer-sorting procedure for inverting functions. M. V. Mathews, B. Atal, J.J. Chang, and J.W. Tukey (Bell Laboratories, Murray Hill, NJ 07974)

For, $\bar{y}=f(\bar{x}), \bar{x}$ and $\bar{y}$ being vectors, we assume $\bar{y}$ may be computed from $\bar{x}$, but $\bar{x}=f^{-1}(\bar{y})$ cannot be computed directly and $\bar{x}$ may be multivalued. An important example is the vocal tract function where $\bar{x}$ deseribes the articulation and $\bar{y}$ describes the resulting sound. Inversion by sorting consists of computing $\bar{y}$ for many values of $\bar{x}$ and sorting the resulting $\bar{y}, \bar{x}$ pairs into a convenient order according to $\bar{y} . \bar{x}$ corresponding to a given $\bar{y}$ is then obtained by looking up $\bar{y}$ in the sorted data. Multiple values of $\bar{x}$ will be adjacent in the sorted data. Subspaces in $\bar{x}$, denoted fibers, may map onto a single $\bar{y}$.
There may be forbidden regions in $\bar{y}$ which cannot be reached by any $\bar{x}$. The local nature of $f(\bar{x})$ including the fibers and forbidden regions are determined by linearization in a neighborhood. Larger regions are computationally explored by extending the linear neighborhoods in sroall steps.

\section{50}

HH12. A rticulatory compensation: A study of ambiguities in the acoustic-articulatory mapping. B Atal, J.J. Chang, M. V. Mathews, and J. W'. Tukey (Bell Laboratories, Murray Hill, NJ 07974)

We present a method of constructinis various vocal-tract shapes having identical acoustical characteristics. A 20-dimensional rocal tract model is used to compute the frequencies, amplitudes, and bandwidths of the first five formants. The dimensions consist of 20 uniformiy spaced cross-sectional areas of the vocal tract. The model ir.cludes losses due to finite wall impedance, glottal leakage, friction, heat conduction, and radiation. A rticulatory regions (fibers) are computed in which the frequencies and amplitudes of the first three formants remain constant. In general these fibers contain 14 dimensions, not all of which p:ovide physiologically reasonable area profiles. A theory fo: selecting among the dimensions within a fiber according tc maximum physical smoothness of the vocal tract is presented. Application of the theory to the 14-dimensional data indicate that 2 or 3 of the 14 dimensions correspond to physically realizable perturbations in the vocal tract and that along these dimensions changes in the vocal-tract shape may be made wi hout changing the three formant frequencies and amplitudes. Almost no audible change in the sound is produced by these articulatory variations. Examples of synthetic speech illust rat ing these results will be played.

\section{1:00}

HH13. Numerical inversion of the trarısformation from articulatory to acoustic parameters in the vocal tract. J.J. Chang, M. V. Mathews, B. Atal, and J.W'. Tukey (Bell Laboratories, Murray Hill, NJ 07974)

A method is presented for determin:ng the parameters of an articulatory model corresponding to a given set of formant frequencies. A four-parameter articulatcory model of the vocal tract was used to define the vocal-tract shape. The articulatory parameters were (a) location of constriction, (b) area of constriction, (c) area of mouth opening, and (d) length of the vocal tract. The model included losse: due to finite wall impedance, glottal leakage, friction, hect conduction and radiation. Frequencies, amplitudes, and br ndwidths of five formants were computed for each of 30720 vocal-tract shapes obtained by uniform sampling along each of the four articulatory dimensions. The data was sorted according to the first three formant frequencies. The articulator positions yielding given values of these formant frequencies can be looked up in the sorted data. One-dimensional fibe:s (curved lines) were computed along which the articulator rositions can vary without producing changes in the three formant frequencies. These fibers span large distances in the artivulatory space. Sounds produced by various articulations along a fiber were computed and were compared by informal listening tests.

\section{1: 10}

HH14. Generalization for damping in the acoustic tube model of speech production. A.H. Gray, Jr., J.D. Markel, and H. Wakita (Speech Communications Research Laboratory, Inc., 800A Miramonte Drive, Santa Barbara, CA 93109)

The widely used method of vocal tract modeling proposed by Wakita [IEEE AU-21 pp. 417-427] is it lossless model which places all possible damping at the glot:is. In addition, the lip radiation impedance for this model is, by definition, zero. Elementary recursion relationships al low unique transformations between the inverse filter $A(z)$ and the acoustic tube areat functions. In this paper, the acoustic tube model is generalized to allow for arbitrary real attenuation within each acoustic 
tube section and to allow for the lip radiation impedance form described by Flanagan ISpeech Analys is (Springer-Verlad, Berlin, 1972), 2nd ed. p. 36l. The approach suggested in Markel and Gray [Linear Prediction of Speech (SpringerVerlag, Berlin, 1976), p. 88] is pursued here and it is shown that a recursive expression for a new inverse filter can be obtained in terms of the area functions, the glottal termination, the internal losses, and a realistic lip radiation impedance. Theoretical and experimental results will be discussed.

$$
\text { 11:20 }
$$

HH15. A better LASS. D. Lloyd Rice (Phonetics Lab Linguistic Department, UCLA, Los Angeles, CA 90024) The first Line Analog Speech Synthesizer (LATSH used at UCLA modeled a vocal tract consisting of 18 sections, each with an area and an acoustic loss factor that can be specified independently. For computational reasons each section had to be the same length, which was obviousty a disadvantage. Another weakness was that the radiation impedance at the lips was modeled in a somewhat simplistic manner. Both these disadvantages have now been overcome, with thut losing the advantage of a model in which the losses can be specified at each point along the tract. A curve is interpolated between specified points along the tongue, converted to areas and smoothed to determine 18 evenly spaced sections. The tosses at the lips are nowịmodeled by a realistic resistive-induative load. The formants calculated using this model have been" compared with those calculated by the previous model, and with those calculated by a model described by Fant. There are still differences between calpolated and observed formant frequencies. We do not know how to decide whether the difficulty in reproducing observed formant frequeses is due to inaccurate estimates of the acoustic losses along the tract or errors in the conversion from sagittal dimensions to crosssectional areas, or some other factors.

$$
\text { 11:30 }
$$

HH16. A coustic effects of longitudinal displacement in vocal- Sord vibration. K. Ishizaka and J.L. Flanagan (AcoureSearch Department, Bell Laboratories, Murray ill, to
07974)

Previously we modelled vocal-cord vibration by a nonlinear mechanical oscillator composed of two stiffness-coupled masses. The oscillator is driven by a fundtion of intraglottal pressure and subglottal pressure, and the coupled mases are permitted one-dimensional displacement transvers to the direction of glottal flow. This formulation produces realistic self-oscillatory behavior, and duplicates characteristic phase-differences in cord motion and acoustic interactions with vq $\mathrm{l}$ thet and subglottal system. Through approximating calculations, we estimated that the longitudinal component of real cord motion (along the direction of glottal flow! has second-order influence upon glottal flow. In this paper we examine more rigorously this earlier estimate. We-modify the twomass model for an additional (longituding thogree of freedom in cord displacement. Oscillator and coupl hig parameters remain as before, but the oscillator is driven by functions of both transglott in pressure and intraglottal pressure. As a result of interaction with the subglottal systen, we observe a microstructure in subglottal pressure variation. The microstructure is excited mainly by vocal-cord closure, and is consistent with measurements on human phonation. Also, we compute dynamic two-dimensional trajectories for the colds (masses), and find that they correspond with observations made on natural larynxes. We conclude that the longitudinal component of displacement is not essential for realistic selfoscillation of the cord model. Finally, we synthesize voiced sounds with the model, and find that the glottal flow component deriving from longitudinal displacement is physically and perceptually of second-order importance.

\section{$11: 40$}

HH17. Study of variations in the male and female glottal wave. R. B. Monsen, A.M. Engebretson, and N.R. Vemula (Central Institute for the Deaf, St. Louis, MO 63110)

Observations of glottal sound wated generated by five mâle and five femalo adults were mad 4 ith a reflectionless tube. Speakers phonated a neutral vowèl in normal, loud, and soft voice and in linguistically relevant syllables: monosyllables with interrogative and declarative intonation and three-syllable words with primary stress on one syllable. Two types of variation of the glottal waveform are observed, depending upon the sex of the speaker, the mode of phonation, and the linguistic context in which phonation is produced. In one case, the harmonic relations of the glottal spectrum remain the same over changing $F_{0}$. In a different type of change, the harmonic relations decline more steeply with increasing $F_{0}$ and the distribution of energy tends to remain constant. These results are compared with glottal waves generated by the FlanaganIshizaka two-mass model of the vocal folds in which subglottal alr pressure and fold tension may be systematically varied. Comparison indicates that in declarative syllables, $F_{0}$ change may be due primarily to declining air pressure, whereas in interrogatiqu syllables, it appears due mainly to increased fold tension. [Supported by NINCDS Grant 03856. ] 


\title{
Session II. Psychological and Physiological Acoustics V: Precis Poster Session on Cochlear Anatomy and Chemistry; Psychophysics A
}

\author{
George Moushegian, Chairperson \\ Callier Center for Communications Disorders, Dallas, Texas 75235
}

Poster Session

$9: 00$

II1. Growth patterns of the peripheral axons of the spiral neurons in culture. H. M. Sobkowicz, J. E. Rose, and B. Bereman (Departments of Neurology and Neurophysiology, University of Wisconsin, Medical School, Madision, WI 53706)

Segments of the organ of Corti with its innervation preserved were excised from the newborn mouse and studied in culture. The following growth patterns were observed: (a) maintenance of the normal innervation pattern and growth along normal lines if the hair cells remain in their usual location, (2) growth by elongation of the fiber if the hair cells migrate into the outgrowth zone and if the fiber remains in contact with the receptor, and (3) free growth of the fiber if its connections with the hair cells are broken. Growth by elongation is confined to the area of hair cell displacement. The growth is well organized and the length of the fiber compensates strictly for the distance the respective hair cell has migrated. Free growth proceeds at a faster rate then does growth by elongation, is independent of the receptors, is un restricted in length, and uncontrolled in amount. The growth is disorganized and luxuriant for at least several weeks after explanation. (Work supported by $\mathrm{NIH}$ grants NS08626 and NS12732.]

\section{9:04}

I12. Horseradish peroxidase ototoxicity. M. Ross (Department of Anatomy, University of Michigan, Ann Arbor, MI 48170), A. Nutall (Kresge Hearing Research Institute, University of Michigan, Ann A rbor, MI 48170), and C. G. Wright (Callier Center for Communication Di sorders, University of Texas at Dallas, Dallas, TX 75235)

Following intracochlear perfusion of horseradish peroxidase (HRP) in artifical perilymph ( $1 \%$ for 10 min-4 $h ; 1-24$ survival), HRP was demonst rated histochemically in some hair cells and supporting cells, the basilar membrane, spiral limbus and stria vascularis of 18 anesthetized (DLAL) guinea pigs. No neuronal retrograde transport occurred. To test for possible HRP ototoxicity, cochlear microphonics (CM) were recorded during presentation of two pure tones $(4 \mathrm{kHz}$, $500 \mathrm{~Hz}$ ) in four HRP experiments and two controls perfused with artificial perilymph alone. Only six drops of perfusate were used in one HRP and one control animal. No significant variation in CM occurred during perfusion. At stop-flow, CM declined slightly but soon stabilized in both 6-drop animals; decline was greater upon stop flow of either perfusate at one hour, but was greatest after HRP. An animal was then perfused with $10 \%$ HRP for one hour; CM declined during perfusion and continued downward at stop flow. Our results indicate ototoxicity of HRP, especially when concentrated, perhaps due to an adverse effect on inner ear fluid balance. Studies which utilized HRP as a tracer in the cochlea should be reassessed.

\section{9:08}

II3. Permeability of cochlear partition to potassium and sodium ions. Teruzo Konishi, P.J. Walsh, P. E. Hamrick, and A.H. Yankwich (National Institute of Environmental Health Sciences, Research Triangle Park, NC 27709)
Permeabilities of guinea pig cochlear partitions were determined by perfusing scala vestibuli (SV) and/or scala tympani (ST) with artificial perilymph with or without ${ }^{22} \mathrm{Na}$ and ${ }^{42} \mathrm{~K}$ tracers. All concentrations of tracer:s were measured by gamma ray spectrometry. Perfusions ranged from 5 to $60 \mathrm{~min}$ at a rate of $2 \mu 1 / \mathrm{min}$. Under these conditions stimulus-related responses and endocochlear potentlal were not greatly suppressed. Perfusions were performed under the following conditions: perfusion of both SV and ST $w$ ith tracers, perfusion of SV with tracers and ST without tracers, and perfusion of SV without tracers and ST with tracers. Results were similar under all the above conditions with concentration of ${ }^{42} \mathrm{~K}$ in endolymph becoming greater than in rerilymph after 5 min of perfusion and becoming five times greater after $30 \mathrm{~min}$ of perfusion. On the other hand ${ }^{22} \mathrm{Na}$ concentration is endolymph was less than $2 \%$ of that in perilymph for all perfusion times. Perfusion of SV and ST with $10^{-4} \mathrm{M}$ ouabain or under anoxia resulted in a decrease of ${ }^{42} \mathrm{~K}$ and an increase of ${ }^{22} \mathrm{Na}$ concentration in endolymph. These findings suggest that active transport of $K$ into endolymph is through stria vascularis.

\section{9:12}

I14. Effects of putative transmitters on afferent cochlear transmission. R. P. Bobbin (Kresge Flearing Research Laboratory, Louisiana State University Medical Center, Department of Otorhinolaryngology, 1100 Florida Ave., Bldg. 164, New Orleans, LA 70119)

Putative transmitters and related sbstances were perfused through the guinea pig scala tympani while monitoring compound action potentials (AP) of the cochlear nerve and cochlear microphonics (CM). The following substances had no effect on the CM or AP when applied at $10 \mathrm{mM}$ : GABA, glycine, $L-$ alanine, $\beta$-alanine, histamine, norepinephrine, taurine, dopamine, and sodium acetate. Substances which had no effect on $\mathrm{CM}$ or AP at $1 \mathrm{~mW}$ but reduced the AP at $10 \mathrm{~mW}$ were glutamate, aspartate, citrate, 5-hydroxytryptamine, tyramine, and bicuculline methiodide. At $1 \mathrm{mM}$, the prostaglandins $E_{1}$, $E_{2}$, and $F_{2} \alpha$ reduced the AP with no effect on the CM while $F_{2} \beta$ and $A_{1}$ had no effect on the AP or CM. The mechanisms underly ing the action of these substan ses is unknown. However, the results seem to eliminate some substances as afferent transmitters and suggest others. [Supported by NIH, USPHS Grant NS-11647-02.]

II5. Abstract withdrawn. 


\section{9:20}

I16. Behavioral assessment of kanamycin-induced hearing loss in the guinea pig. M. R. Petersen, C.A. Prosen, D. B. Moody, W.C. Stebbins, and J. E. Hawkins, Jr. (Kresge Hearing Research Institute and the Departments of Psychology and Otorhinolaryngology, University of Michigan, Ann Arbor, MI 48109)

The effect of chronic kanamycin administration on absolute auditory thresholds measured with the operant conditioning procedure described in the previous paper was examined in four guinea pigs. The absolute threshold for five different frequencies was measured daily, thus permitting construction of an audiogram which ranged from $125 \mathrm{~Hz}$ to $34 \mathrm{kHz}$ within a two-day period. Following establishment of a predrug baseline the animals received $200^{\circ}-\mathrm{mg} / \mathrm{kg}$ injections of kanamycin daily for 15 to 30 consecutive days. In the course of drug treatment the animals suffered permanent hearing losses which progressed gradually from the higher to the lower frequencies. The maximum threshold shift observed at any of the test frequencies was approximately $50 \mathrm{~dB}$. In fact, shifts in absolute threshold at frequencies most severely affected by the drug were within a range of $35-50 \mathrm{~dB}$. The correspondence between the hearing impairments determined behaviorally and the cytocochleograms constructed from light microscopic examinations of surface preparations of the organ of Corti will be discussed. [Supported by Research Grants NS05077 and NS05065 and by Program Project Grant NS05785 from NINCDS. 1

\section{$9: 24$}

II7. Patterns of activity in single auditory nerve fibers of the goldfish. R. R. Fay (Department of Psychology, Loyola University of Chicago, 6525 N. Sheridan Rd., Chicago, IL 60626)

The response of single fibers of the goldfish saccular nerve were recorded extracellularly in response to tonal stimuli. For each unit studied, quantitative measures of phase-lockking (period histograms) were made in response to several tonal frequencies, each presented at a wide range of sound pressure levels. The sensitivity and responsiveness of each unit were plotted as functions of frequency in terms of both spike rate modulation, and phase-locking criteria. Fibers showing little adaptation, high spontaneous firing rates, and low-pass frequency response characteristics were designated type $\Pi$ fibers, while those showing less spontaneous activity, greater adaptation and high-pass frequency characteristics were designated type I fibers, to correspond to an earlier classification [T. Furukawa and Y. Ishii, J. Neurophysiol. 30, 1377-1403 (1967)]. Within each classification, sensitivity, spontaneous activity patterns, phase-physical data on frequency analysis suggest that it is in the temporal patterns of spike activity, and not in the spatial patterns of spike rates that most auditory information is conveyed to the brain. [Work supported by the NSF.]

\section{$9: 28$}

II8. Effects of temperature on the response properties of auditory nerve fibers in the American toad (Bufo americanus). A.J.M. Moffat and R. R. Capranica (Section of Neurobiology and Behavior, Cornell University, Ithaca, NY 14853)
While a number of studies have explored the effects of temperature on the electrical potentials in the inner ear, little is known about such effects on auditory nerve fibers. We therefore conducted a study of changes in the response properties of single auditory units in the VIIIth nerve of Bufo americanus as the animal's temperature was varied. Toads are opportune animals for such studies since they can tolerate a wide range of body temperature. While recording continuously from an individual fiber, the toad's temperature was varied by. a Peltier plate to which it was secured. A total of 27 units in 4 animals was studied. As the animal's temperature was lowered, the best excitatory frequencies (BEF) of fibers from the amphibian papilla shifted downwards while their thresholds increased. In addition, both spontaneous activity and maximum firing rate to a tone at a unit's BEF decreased although its dynamic range remained approximately $30 \mathrm{~dB}$ above threshold. Fibers from the basilar papilla also showed a diminution in spontaneous activity and maximum firing rate. However, their BEF's and thresholds changed very little with temperature. These results suggest that the basis for frequency analysis differs between the two organs.

\section{9:32}

II9. Temperature effects on single auditory nerve fiber responses. R. A. Eatock and G. A. Manley (Biology Department, McGill University, Montreal, Quebec, Canada H3C 3G1)

Single-fiber activity in the cochlear nerve of Gekko gecko was monitored while raising the body temperature. Pure tone thresholds were measured repeatedly as the temperature rose until contact with the fiber was lost. Temperatures were monitored orally with a small sensor next to the contralateral cochlear duct. Frequency-threshold tuning curves of all fibers consistently shifted towards higher frequencies at higher temperatures. Temperature increments as small as $1^{\circ} \mathrm{C}$ raised a fiber's characteristic frequency (CF) by 50 to $150 \mathrm{~Hz}$, and frequently augmented its sensitivity at CF by several $\mathrm{dB}$ SPL. The whole tuning curve shifted towards higher frequencies but did not significantly change shape. These changes are consistent with temperature effects on lizard cochlear microphonic [Y.L. Werner, J. Exp. Zool. 195, 319-352 (1976)], summating potential of mammals [J. A. Manley and B.M. Johnstone, J. Comp. Physiol. 88, 43-66 (1974)] and evoked potentials from lizard cochlear nuclei (H.W. Campbell, Physiol. Zool. $42,183-210(1969)]$. The significance of these data for singleunit work and basilar membrane motion measurements is discussed.

$$
9: 36
$$

I10. Call discrimination as a function of playback intensity in the green treefrog (Hyla cinerea). H. Carl Gerhardt (Division of Biological Science, University of Missouri, Columbia, MO 65201)

The intensity at which female green treefrogs (Hyla cinerea) detected spectral and fine-temporal changes in synthetic vocal signals varied. Given a simultaneous choice between a standard call consisting of three components $(0.9+2.7+3.0 \mathrm{kHz})$ of equal relative amplitude and a call in which the two high-frequency components were attenuated by $15 \mathrm{~dB}$, females consistently chose the standard call at 65,75 , and $85 \mathrm{~dB}$ but failed to discriminate at $60 \mathrm{~dB}$. The same results were obtained when females were given a choice between the standard call and one in which the $300 / \mathrm{sec}$ beating was lacking (i. e., $0.9+3.0 \mathrm{kHz}$ ). Females discriminated in favor of the standard call at 60 , 65 , and $75 \mathrm{~dB}$ when the low-frequency component of the alternative stimulus was attenuated by $10-\mathrm{dB}$ relative to the two high-frequency components. These results will be discussed in relation to response properties of the peripheral auditory system and to the ways in which intensity and spectral properties of natural and synthetic calls change as a function of distance, habitat, and elevation of the sound source. [Work supported by NSF. 1 


\section{9:40}

II1. Comparison of the response of the two-fiber populations in the auditory nerve of the alligator lizard. R. G. Turner and D. W. Nielsen (Otological Research Laboratory, Henry Ford Hospital, Detroit, MI 48202) and D.C. Teas (Institute for the Advanced Study of Communication Processes, University of Florida, Gainesville, FL 32611)

The basilar papilla of the alligator lizard (Gerrhonotus multicarinatus) contains two anatomically distinct hair cell populations. The apical population has unidirectional orientation and is associated with a tectorial membrane. The dorsal population has bidirectional orientation and free-standing eilia. On the basis of tuning curve properties, the primary auditory nerve fibers have been eategorized into low $(<0.9 \mathrm{kHz})$ and high $(>0.9 \mathrm{kHz}) \mathrm{CF}$ populations. These two populations have been associated with the apical and basal regions of the papilla, respectively (Weiss, Mulroy, Turner, and Pike, J. Acoust. Soc. Am. 55, S84(A) (1974)]. The objective of the present study was to determine if other measures of the physiological response of the fibers could distinguish the two fiber populations. Action potentials were recorded from single, primary auditory nerve fibers in response to click, tone burst, and noise burst stimuli. The data indicate significant differences in the response of the two-fiber populations to the same acoustic stimulus. It is proposed that these physiological differences result primarily from the anatomicil differences of the associated hair cell populations. IWork supported, in part, by NIH grants NS07287, NS06459, and NS05475, the Deafness Research Foundation, and Henry Ford Hospital. 1

$$
\text { 9:44 }
$$

II12. Estimation of the length of the traveling wave envelope from response areas of neurons in the cochlear complex of the cat. J. E. Hind, J. E. Rose, M. M. Gíbson, and L. M. Kitzes (Department of Neurophysiology, Univ. of Wisconsin, Madison, WI 53706)

Plots of discharge rate vs frequency at a number of intensities were determined for 163 neurons. Presumably the bandwidth of these response areas is determined by the shape of the traveling wave envelope on the basilar membrane. According to Greenwood, the distance from the peak to the apical foot of the wave is approximately constant [D. D. Greenwood, J. A coust. Soc. Am. 34, 1364-1369 (1962)]. Using his frequencydistance function for the eat, we have calculated for each response area the distance along the basilar membrane between the locations for the best frequency and the upper cutoff frequency. When this "critical distance" (c. d.) is plotted versus best frequency for all units, c. d. tends to increase as best frequency declines below about $2 \mathrm{kHz}$. In order to obtain relatively constant $c$. $d$. throughout the entire frequency range, it is necessary to revise Greenwood's function such that the distances for the lower octaves deviate even more from a logarithmic representation than suggested by his original curve. [Supported by NIH grants NS 06225 and NS 12732.]

\section{$9: 48$}

II13. Effects of stimulus frequency on two-tone suppression. P.J. Abbas (Department of Speech Pathology and Audiology, University of Iowa, Iow a City, IA 52242)

Effects of stimulus frequency on two-tone suppression were investigated in single auditory-nerve froers of anesthetized cats and compared with human psychophysical data. Discharge rate of single fibers was plotted as a function of stimulus level of a tone $\left(f_{1}\right)$ both alone and in the presence of a second tone $f_{2}$. $f_{2}$ was always well below the fiber's characteristic frequency and was held at a fixed sound pressure level appropriate to produce two-tone suppression. Rate functions for the two-tone stimulus were thus depressed or shifted horizontally with respect to the single-tone $\left(f_{1}\right)$ rate function. When $f_{1}$ was varied with $f_{2}$ fixed, suppression was maximum with $f_{1}$ at fiber $C F$. A psychophysical experiment was also conducted with stimulus parameters similar to those above. In this case, however, the two-tone stimulus was used in a forward-masking paradigm similar to that used by Shannon [J. Aroust. Soc. Am. 59, 1460 (1976)). The unmasking effect as a function of frequeney and level was compared to the single-fiber data. [Research supported by NIH Grant FR-07035. ]

\section{9: 52}

II14. Forward and simultaneous tonal suppression of singlefiber responses in the chinchilla auditory nerve. D. Harris, P. Dallos, and N. Kraus [Auditory Plyssiology Laboratory (Audiology), Northu'estern University, Evanston, IL 60201]

Response patterns (PST histograms) of auditory nerve fiber discharges with two-tone stimuli were obtained. The two tones either overlapped (simultaneous suppression) or were temporally separated (forward suppression). The response elicited by a probe tone at the fiber's CF decreases when immediately preceded by a masking tone. It was found that the frequency/ intensity combinations of effective maskers lie within the tuning curve of the fiber. In simultaneous two-tone suppression the frequency/intensity contours of the masker are broader than those observed in the forward suppression condition. The post stimulus suppression in firing below spontaneous rate, which is typically observed following cuaintained excitat ion, is suggested as the single-fiber substrate for forward suppression and masking effects observed in $\mathrm{AP}$ and psychophysics. [Supported by Grants from the NNCDS.]

\section{$9: 56$}

II15. Speech perception by the chinchilla: Categorical perception of synthetic alveolar plosive consonants. Patricia K. Kuhl (Central Institute for the Deaf, 818 South Euelid, St. Louis, MO 63110)

Kuhl and Miller [Science 190, 69-72 (1975)] recently demonstrated that in an identification task the chinchilla responds to ("labels") an alveolar synthetic-speech continuum as though an abrupt qualitative change occurs at the place where english speakers hear a change from $/ \mathrm{da} /$ to $/ \mathrm{ta} /$. Discrimination functions for these same stimuli have now been obtained. The animal's task is to detect a change from a repeating "standard" stimulus to a "comparison" stimulus and report that change by crossing a midline barrier to avoid shock. The standard stimuli are $0,+10,+20,+30, \div 40,+50,+60,+70$ and +80 msec VOT. The VOT value of the comparison stimulus is gradually changed until it approaches the VOT ralue of the standard. The animal's threshold (the minimum $\Delta t$ detected with $75 \%$ accuracy) is measured for each standard stimulus and is best when the standard is $+30 \mathrm{msec} V O T$, that is, at the animal's perceptual boundary as defined in the labeling experiments. This agreement between the labeling and discrimination data demonstrates categorical perception for a voiced-roiceless continuum by an animal listener. [Supported by NIH grant NS 03856 to CID. ]

II16. Intensity functions of cortical auditory neurons in the awake monkey. Thomas A. O'Connor (Department of Neurophysiology, 283 Medical Sciences Building, University of Wisconsin, Madison, WI 53706) and Bryan E. Pfingst and Josef M. Miller (Department of Otolaryngology and Regional Primate Research Center, University of Washington, Seattle, WA 98195)

The relation between stimulus intensity and single unit activity was examined in the auditory cortex of unanesthetized monkeys trained to perform in an auditory reaction time task. Data was collected primarily under performance conditions, but nonperformance data was also acquired. Subsequent histology indicated that most recorded units were located in koniocortex and the lateral and caudal parakoniocortices ID. N. Pandya and F. Sanides, Z. Anat. Entwickl.-Geseh. 139, $127-161(1973)]$. In $70 \%$ of the units examined, discharge rate was a nonmonotonic function of stimulus intensity at best frequency. Thirty percent of the units had monotonic intensity functions. This proportion did not vary substantially with cortical location. Monotonic functions tended to rise less steep- 
ly from threshold as intensity was increased than did nonmonotonic functions. Monotonic functions also exhibited a wide spread of dynamic ranges, approximately half of which exceeded $50 \mathrm{~dB}$. Dynamic ranges as great as $80 \mathrm{~dB}$ were seen in some monotonic units. Quantitative aspects of intensity functions, including slope, threshold, and discharge rate, are discussed as they relate to monotonic and nonmonotonic functions. Implications with respect to intensity coding in the cortex are also discussed. [Work supported by NIH Grants NS-08181 and GM-00260.I

\section{0:04}

II17. Latency changes for unit responses at rat inferior colliculus after early auditory deprivation. B.M. Clopton and M.S. Silverman (Department of Otolaryngology, School of Medicine, University of Washington, Seattle, WA 98195)

A decrease in the latency of neural responses is a commonly reported concomittant of development in the auditory system. We investigated the effect of sound deprivation on the developing auditory system of rats by observing latency changes in unit responses at the inferior colliculus following deprivation. Ligation of one external meatus at ten days after birth produced partial sound deprivation until the ligation was removed two to five months later. Units at both colliculi were studied by acute recording to determine characteristic frequency (CF) and initial spike latencies for clicks of different intensities. At $30 \mathrm{~dB}$ above click threshold most units with high CF's that were activated by the deprived ear had response latencies two to three times the latencies for units of comparable CF's driven by the undeprived ear. Units with CF's below $10 \mathrm{kHz}$ had essentially normal latencies. Observations at other sites indicate that neural, not mechanical changes are responsible for these findings. [Work supported by NIH Grant NS1.3052.1

\section{0:08}

II18. Critical period for the development and maintenance of binau ral interaction. M.S. Silverman and B. M. Clopton (Department of Otolaryngology, School of Medicine, University of Washington, Seattle, WA 98195)

A determination of the critical period for the development and maintenance of binaural interaction was made by unit recording at the inferior colliculus of neonatal and sound deprived rats. Collicular un its are activated by clicks delivered to the contralateral ear. An indication of binaural interaction in normal adult rats is the suppression of this activity by ipsilateral clicks that are of equal or greater intensity. Such binaural interaction was evident in the unit activity of young rats during the first week after the onset of hearing. Monaural deprivation beginning at 10 days after birth (DAB) and continued for three to five months abolished binaural interaction. The same duration of deprivation had much less effect if begun at 30 days and no effect if initiated at 60 days. The result indicate that the essential connections for binaural interaction are genetically predetermined and present at the onset of hearing at $10 \mathrm{DAB}$. The critical period for experiential validation of binaural interaction begins at the onset of hearing and extends up to $60 \mathrm{DAB}$. These conclusions should be considered specific to the rat. [Work supported by NIH Grant NS-13052.]

\section{$10: 12$}

II19. Developmental changes of susceptibility to temporary threshold shift in hamsters. G. R. Bock (Department of Otorhinolaryngology and Human Communication, University of Pennsylvania, Philadelphia, PA 19174)

The magnitude of temporary threshold shift (TTS) and the time course of recovery of TTS were studied in young hamsters. Animals aged 15, 28, 40, 55, or 85 days were anesthetized with urethane and a sound speculum was sealed around the left tympanic ring. Eroked potentials in response to tone bursts were recorded from an electrode in the right inferior colliculus and thresholds for detecting the computeraveraged neural response were measured before and for 100 min after a 10 minute presentation of a continuous tone $(3 \mathrm{kHz}$, $110 \mathrm{~dB}$ SPL). Mean threshold shifts at $4 \mathrm{kHz}, 1$ min postexposure, were $16,35,41,28$, and $25 \mathrm{~dB}$ in the 15-, 28-, 40-, 55-, and 85-day-old groups respectively. Threshold shifts recovered within $100 \mathrm{~min}$ in $15-$ and 85 -day-old animals, but required considerably longer to recover in the other age groups. The data suggest that young hamsters pass through a critical period of susceptibility to TSS. Consideration of the development of normal thresholds suggests that the critical period may be dependent on developmental changes within the cochlea. (Work supported by the Deafness Research Foundation. ]

\section{0:16}

II20. Decay of the acoustic reflex during a prolonged exposure to noise. Kenneth J. Gerhardt (School of Speech, Kent State University, Kent, OH 44242) William Melnick (Department of Otolaryngology, Ohio State University, Columbus, $\mathrm{OH}$ 43210) and John A. Ferraro (Department of Communication, Ohio State University, Columbus, OH 43210)

Decreases in sound attenuation resulting from acoustic arousal of the middle-ear-muscle reflex were investigated during an 8-h exposure to an octave band of noise centered at $0.5 \mathrm{kHz}$ at $95 \mathrm{~dB}$ SPL. Chinchillas were chronically implanted with round-window recording elect rodes. Changes in the amplitude of the cochlear microphonic during continuous noise exposure reflected activity of the acoustic reflex (AR). The 8-h exposure was composed of four consecutive 2-h periods separated by 11-min "quiet" intervals. The AR remained active throughout each of the four exposures, but the magnitude of the reflex decreased approximately $30 \%-50 \%$ within each $2-\mathrm{h}$ period. This decay ing process appeared to reach an equilibrium following 8-30 min of exposure after which a constant level of effect was maintained. The initial magnitude of attenuation measured after noise onset decreased with each additional exposure period.

$10: 20$

II21. Neural correlates of auditory plasticity during classical conditioning in the rabbit. B. L. Lonsbury-Martin, G. K. Martin, S. Schwartz, and R. F. Thompson (Department of Psychobiology, University of California, Irvine, CA 92717)

Multiple units were recorded from auditory st ructures during the classical conditioning of the rabbit's nictitating membrane response. Stainless steel insect pin electrodes $(50 \mu \mathrm{m})$ were used to record bilaterally from small groups of cells in either the cochlear nucleus, inferior colliculus, or medial geniculate. Although all electrode placements responded to the white noise conditioned stimulus, most of these responses were labile in that peristimulus-time histograms indicated considerable variability in the response patterns of the cell groups over trial blocks. In general, onset and sustained responses to the conditioned stimulus tended to habituate during the 117-trial acquisition session. However, a number of subjects with medial geniculate or inferior colliculus placements showed an increase in the sustained response to the conditioned stimulus associated with acquisition of the learned response. The relationship of the observed neural changes to the anatomical location of electrode placements is considered. [Supported by grants from NSF and NINCDS. ] 


\title{
Session JJ. Noise VI. Community Noise Assessment, Measurement and Monitoring
}

\author{
Karl S. Pearsons, Chairperson \\ Bolt Beranek and Newman, Inc., Canoga Park, California 91303
}

\section{Contributed Papers}

\section{$8: 30$}

JJ1. Politics and noise control. R. Way and A. E. Perez (Minnesota Pollution Control Agency, 1935 West County Rd. B2, Roseville, MN 55113)

The early 1970's saw a proliferation of noise control legislation at all levels of government in this country. Many of these efforts failed and continue to fail, amid continuing public support, as a result of the political system. The 1971 Minnesota Legislature mandated the Pollution Control Agency (PCA) to control noise that "may be injurious to human health or welfare, animal or plant life, or property, or could interfere unreasonably with the enjoyment of life or property." This mandate is supported by one of the most comprehensive examples of noise control legislation in existence. The PCA in responding to the mandate was faced with a State political system burdened with past pollution control legislation, and leery of major commitments to a program which lacked the federal support of previous efforts. In addition, the political system was under pressure from powerful political lobbies to stop any further environmental programs. The noise control program is now in its maturing stages, with results toward a better environment becoming evident. The legislative mandate and historical key events of the program will be discussed.

\section{$8: 45$}

JJ2. Community response to aircraft noise: level versus number relationship. William Connor and Harrold Patterson (Tracor, Inc., 6500 Tracor Lane, Austin, TX 78721)

Response behavior in terms of a typical scale of individual annoyance was examined for various categories of level and number of aircraft operations. The data base was obtained from social surveys and acoustical measurements around nine airports in the USA during the period 1967-1971. For the sample as a whole, adverse response tended to increase with the energy-mean perceived noise level of aircraft operations; in relation to numbers of operations, however, response tended to peak in the $100-200$ operations per day category rather than increase monotonically. This result does not conform to certain accepted response models, suggesting a need to develop and test other models. [Work supported by NASA.]

\section{9:00}

JJ3. Establishing noise criteria for residential living in areas surrounding commercial aviation airports. J. E. Mabry (MANAcoustics and Noise, Inc., 2105 N. 45th St. , Seattle, WA 98103)

Results are provided which contribute to establishment of noise levels that are compatible with residential living activities. Community-noise simulation systems were placed in the homes of 24 families that were not impacted by airport noise. Each family was exposed to four different airport noise conditions of one week duration. Interference with daily living activities and annoynace responses to the four conditions were obtained. Some results and conclusions are as follows: (1) Noise from other sources within their homes bothered or annoyed the participants as much or more than the flyover noises. (2) Behavioral awakening did not occur for many participants exposed to night flyovers peaking at approximately
$70 \mathrm{~dB} A$. It was concluded that the $10 \mathrm{~dB}$ penalty applied to night flyovers for NEF and $L_{c h}$ is too large. (3) For the mix and number of aircraft utilized, it was concluded that a NEF of 32 to 33 is compatible with indoor residential living. [FAA-RD supported.]

JJ4. Variation of community noise sensitivity with time of day. J. B. Ollerhead (Department of Transport Technology, University of Technology, Loughborough, Laicestershire I.E11 3TU, England)

It is widely accepted that people are more annoyed by noise during the evening and nighttime hours than during the day period. Accordingly, many community-noise rating indices involve a division of the $24-\mathrm{h}$ day into two or three time periods, attaching different weights to events in each. Unfortunately, it has proved very difficult to obtain quantitative measurements of these weightings through social survey studies, and consequently the weightings adopted tend to be based mostly upon intuitive judgments. This paper descrtbes an attempt to obtain direct measurement of relative sensitivity to noise during the three periods. The measurements, which were made as a part of a wider study of the aireraft noise problem, support current estimates of inc reased sensitivity during the evening, but indicate a surprising immunity to noise during the night.

$$
9: 30
$$

JJ5. TTS from irregular bursts of noise. W.D. Ward, E. M. Cushing, and E. M. Burns (Hearing Research Laboratory, University of Minnesota, 2630 University A ve. SE, Minneapolis, MN 55414)

Most studies of the TTS produced by noise have used regular interruptions of the noise; in general, such studies have shown that the TTS is proportional to the on fraction. However, such regularity is not characteristic of most industrial settings. In order to assess the effect of variability in burst and pause durations, the TTS produced in 10 young normal-hearing adults by 6-h exposures to magenta noise at $100 \mathrm{~dB} A$, using an average on fraction of $50 \%$ but with four different temporal patterns, was measured. Exposure patterns were: (1) on $3 \mathrm{~min}$, off 3 min during entire run; (2) on either 1 or 5 min $(50 \%$ probability), off either 1 or $5 \mathrm{~min}$, burst and jause durations independently selected; (3) on and off 1 or 5 min, pause duration same as preceding burst duration; and (4) on and off 1 or $5 \mathrm{mln}$, pause duration different from preceding burst duration. No difference in TTS measured 2 or 30 min after exposure was found (maximum average TTS $_{2}$ was $8 \mathrm{~dB}$ at $4 \mathrm{kHz}$ ), indicating that an intermittent noise can be adequately characterized by its average on fraction for purposes of exposure standards. [Research supported by the National Institute for Occupational Safety and Health, Public Health Servi.ce. J

\section{$9: 45$}

JJ6. The annoyance and loudness of magenta noise. E.M. Burns, C.W. Turner, and W. D. Warid (Hearing Research 
Laboratory, University of Minnesota, 2630 University Ave. S. E., Minneapolis, MN 55414)

The relative "annoyance" and "loudness" of magenta noise (a broadband noise whose octave-band spectrum falls off at the rate of $5 \mathrm{~dB}$ per octave) and truncated-magenta noise (the high-frequency portion of the magenta noise) were determined using a $2 \mathrm{AFC}$ procedure. For moderate intensities $(90 \mathrm{~dB} A$, magenta) the relative annoyance and loudness were qualitatively in the direction predicted by the PNdB and Sone scales, respectively. That is, the magenta noise was judged to be significantly more annoying and louder than the truncatedmagenta noise, for the condition in which the energy in the frequency region common to both noises was equal. However, for the same condition at higher intensities $(110 \mathrm{~dB} A$, magenta) the truncated-magenta noise was judged significantly more annoying, while the magenta was judged louder. Thus at high intensities we have the paradoxical situation in which the deletion of a major portion of the energy from the spectrum results in a considerably more annoying noise, a result which is not predicted by PNAB. Possible explanations for these results, along with their relevance to aircraft noise, are discussed.

\section{0:00}

JJ7. The relationship between aircraft noise annoyance and duration above specified noise levels. T. L. Hughes and J.E. Mabry (MAN-Acoustics and Noise, Ine, , 2105 N. 45th St., Seattle, WA 98103)

Relationships were investigated between community response to airport noise and dwell time noise determination methods. Two community response measures were employed. One involved whether or not the respondent disliked his neighborhood due to aircraft noise and the second measure was concerned with whether or not airport noise was perceived as the "MOST serious problem in this community right now ?" Seven dwelltime levels involving the amount of time that the noise was equal-to-or-greater-than a specified level were investigated. These seven dwell-time levels were 65 to $95 \mathrm{~dB} A$ in $5-\mathrm{dB} A$ increments. A method for completely describing the noise at each respondent's home was developed and utilized. Some conclusions are as follows: (1) The dwell-time approach is an effective method for quantifying aircraft noise impact. (2) Dwell-time determinations based on a particular level do not accurately predict dwell-time determinations at another level. (3) For detailed land use planning, a method involving cumulative noise impact at a particular point has high utility. [FAAEQ supported.]

\section{$10: 15$}

JJ8. A irport noise: A monitoring program and the first steps toward its solution. A. E. Perez (Minnesota Pollution Control Agency, 1935 West County Rd. B2, Roseville, MN 55113)

Airport noise is easily the most intensive, publicized, and emotional noise problem facing urban dwellers. The problem is also a magnificent example of federal inaction, and technical doubletalk as a result of its complexity. The Minneapol isSt. Paul International Airport, even though singular in innovative noise abatement procedures developed under public pressures, cont inued to unacceptably impact the life and property of its neighbors. In facing the problem, the Agency encountered: lack of successful case histories, unmeasurable criteria, unaffordable instrumentation, and noncooperation by federal agencies and industry. Roadblocks were overcome steadily: Noise Standards were adopted in November 1974; automatic noise monitors were developed at reasonable prices (\$200 per unit) and 33 units purchased; a monitoring program with the help of 350 families was implemented, the impact of the problem was defined acoustically and demographically, unique operational characteristics of airline companies uncovered and documented and an objective dialogue with the source started. State Regulations on this problem are being developed to control areas where voluntary compliance is not expected and to supplement recent legislative action.
$10: 30$

JJ9. Noise impact evaluation of a military airbase. E.S. Ivey and G.A. Russell (Department of Mechanical Engineering, University of Massachusetts, Amherst, MA 01002) and A. Hicks (U.S. EPA Region I, Boston, MA 02203)

A 24-h noise survey was conducted in the vicinity of Pease A FB, Portsmouth, NH, using both automatic and manual sampling techniques. The manual sampling procedure used allowed the noise contributions from all military aircraft operations to be distinguished from the residual noise levels. By processing the manually sampled data twice, once with the military aircraft noise signals included and once without, it was possible to define a "with and without noise impact" of the military aircraft operation on the surrounding community. Results of the analysis indicated a good correlation between the manual and automatic sampling methodologies and with CNEL contours previously developed for Pease AFB. Significant differences between the "with and without military aircraft" LEQ and LDN noise levels were found at distances of five miles from the base. The measurement and analysis methodology used in this study provides a unique noise impact assessment tool for community noise studies in the vicinity of operating airports. Although only very simple measurement and data reduction techniques are required, this assessment methodology yields useful "with and without" information. As such, it can be applied in a variety of land use planning situations.

\section{$10: 45$}

JJ10. Development of airport noise contours by extensive noise monitoring and analytical modeling. R. D. Horonjeff (Bolt Beranek and Newman Inc., P.O. Box 633, Canoga Park, CA 91305)

This paper describes one of the first known efforts to combine analytical modeling techniques and extensive field noise monitoring to produce airport noise contours. The objective was to produce community noise equivalent level (CNEL) contours whose accuracy could be enhanced and defended by field measurements. An advanced computer simulation model (developed for the U.S. Air Force) was used in concert with 24-h noise monitor data at 44 locations surrounding Miramar Naval Air Station at San Diego, CA. A three-phase study was undertaken to satisfy the objective: (1) develop a preliminary set of contours using the analytical model and the best available data base, (2) use contours to select noise monitor locations and acquire monitor data, and (3) normalize monitor data to typical flying conditions and update noise contours. The paper discusses the variables involved in generating the contours, their ability to be reliably measured at reasonable cost, and their relative impact on contour accuracy.

\section{1:00}

JJ11. Waterfront land use planning. J.K. Parry and H.J. Parry (Parry Noise Consulting, 3060 N. E. 97th Street, Seattle, WA 98115)

Results of a comprehensive study of industrial land use to achieve maximum potential noise compatability with nearby residental communities are presented. Land use plans developed during the study were concerned with a variety of proposed projects at one large waterfront facility operated by the Port of Seattle and covering the time period of $1976-1980$. During the study both adverse and beneficial noise impacts were identified using the criteria of the existing conditions at the facility and the Washington State Noise Standards. Emphasis in the paper presented is placed on the cost effectiveness of careful land use planning through a tradeoff comparison between different noise-abatement approaches. The results and discussions presented are based on measurements of noises made specifically for this study, during previous waterfront noise studies and those reported in the literature. Proposed and potential uses for the waterfront facility are described in the terminal layout plan along with projected 
construction and facility changes. These include relocation of roads, rail tracks, riil headings, Iocation of ship unloading berths, truck and rail car loading ramps, and building demolitions and replacements.

\section{$11: 15$}

JJ12. Low-cost community-noise monitoring system. J. D. Chalupnik (Department of Mechanical Engineering, FU-10, University of Washington, Seattle, WA 98195)

A system for acquiring the statistical data base required to determine 24-h ambient-noise descriptors such as the daynight equivalent $A$-weighted sound pressure level $\left(L_{D_{N}}\right)$ is described. The system is inexpensive and utilizes standard laboratory instruments extensively. It is designed to take hourly samples of 5-15-min duration on an analog tape recorder. The data is anilyzed in the laboratory on a general purpose digital computer. Results of tests in which the system was compared with an all digital system are given. The system performs as expected and is very easy to use.

$$
11: 30
$$

JJ13. Variables to be considered when predicting the $L_{c c i}$ of blast noise. N. D. Lewis and G. A. L.dz (U.S. Army Environmental Hygiene Agency, Aberdcen Proving Ground, MD 21010)

The $C$-weighted day-night average sound level $\left(L_{c a n}\right)$ is currently being used to evaluate the imp xct of blast noise on nearby communitics. When predicting these $L_{c d n}$ values, several variables must be considered. The difference between the linear peak level and $C$-weighted slow lovel is not eonstant as implied in the criteria, but varies with distance from souree and weapon-charge combination. The prediction of the Ievel must also consider more than spherieal sprcading, it must consider the wind direction and velocity. The time of day when the bIast occurs must also be considered, as temperature inversions, occurring primarily when the surface is cooler than the air above it, cause increased sound levels. A final variable considered is the char $;$ e weight-sound level relationship which also varies between weapons. The effects of these variables on the sound level have been observed in blast noise measurements at distances from 150 to $5000 \mathrm{~m}$.

\title{
Joint Meeting of Standards Committees S1 and S3
}

(The activities of $\mathrm{S} 1$ will be discussed first, proceeding to matters of interest to both $\mathrm{S} 1$ and $\mathrm{S} 3$, and concluding with $\mathrm{S} 3$ activities.)

\author{
G.C. Maling, Jr., Chairperson S1 \\ IBM Corporation, Poughkeepsie, New York 12603
}

Standards Committee S1, Acoustics. Working group chairpersons will report on their progress in the preparation of standards, methods of measurement and testing, and terminology in physical acoustics, electroacoustics, sonics, ultrasonics, and underwater sound. Work in progress includes measurement of noise sources, atmospheric attenuation, roise dosimeters, integrating sound-level meters, and revision and extension of sound-level meter speeifications. Open discussion of committee reports is encouraged. A report from the sl Subcommittee on Noise will be presented.

\section{W. Melnick, Chairperson S3}

\section{Department of Otolaryngology, Ohio State University, Columbus, Ohio 43210}

Standards Committee S3, Bioacousties. The current status of standards under preparation will be discussed. In addition to those topics of interest including hearing conservation, noise dosimeters, hearing aids, etc., consideration will be given to new standards which might be needed over the next few years. A report from the S3 Subcommittee on Noise will be presented. 


\title{
Session KK. Psychological and Physiological Acoustics VI: Precis Poster Session on Noise Effects; Psychophysics B
}

\author{
G.D. Pollak, Chairperson \\ Department of Zoology, University of Texas, Austin, Texas 78712
}

Poster Session

$2: 00$

KK1. Neural firing patterns in the chinchilla cochlear nucleus after noise-induced asymptotic threshold shift. R. Salvi, D. Henderson, and R. Hamernik (Department of Otolaryngology and Communication Sciences, State University of New York Upstate Medical Center, Syracuse, NY 13210)

Chinchillas were exposed to an octave band of noise (CF $=4.0 \mathrm{kHz}, 86 \mathrm{~dB} \mathrm{SPL}$ ) for three to five days. At the cessation of the noise, unit activity was sampled in the dorsal cochlear nucleus and ventral cochlear nucleus over a period of $1-12$ h post-exposure. In previous experiments [J. Mills, J. Speech Hear. Res. 16, 426-438 (1973)], the same exposure produced $40 \mathrm{~dB}$ of asymptotic threshold shift at $2-8 \mathrm{kHz}$ measured behaviorally. Single units having CF's below $1.0 \mathrm{kHz}$ had normal tuning curves while those above $1.0 \mathrm{kHz}$ had thresholds that were elevated, on the average, by $40 \mathrm{~dB}$ and, in some instances, by as much as $70 \mathrm{~dB}$. Tuning curves from the highthreshold units were abnormally broad due to the shallow slope on the low-frequency side of the tuning curves. In some units only inhibitory areas were present, while in others, the threshold for the low-frequency inhibitory areas was considerably lower than for the excitatory area. Spontaneous activity from the high-threshold units appeared to be normal or lower than normal. The single unit results will be related to the postexposure behavioral audiogram and cochleagram.

\section{2:04}

KK2. Effects of noise induced permanent threshold shift on single units in the cochlear nucleus. R. Salvi, D. Henderson, and R. Hamernik (Department of Otolaryngology and Communication Sciences, State University of New York Upstate Medical Center, Syracuse, NY 13210)

Chinchillas were exposed to nonreverberant impulse noise (50 impulses, $1 / \mathrm{min}, 155 \mathrm{~dB}$ p.e. SPL, $1-\mathrm{msec} A$ duration) and allowed to recover for at least 40 days following the exposure. Single neurons were subsequently sampled from the dorsal and ventral cochlear nucleus. Many units from the noise exposed animals had abnormally high thresholds and broad tuning curves. Due to the shallow slope of the low-frequency tail of the tuning curve, some units gave responses that were exclusively inhibitory. In other units, the threshold for the inhibitory area was $30-40 \mathrm{~dB}$ lower than the threshold for the excitation area. Many of the high-threshold units also showed abnormally high rates of spontaneous activity. The results of single unit experiments are compared with the nature and extent of the cochlear lesions obtained using the surface preparation.

2:08

KK3. Patterns of impulse noise-induced TTS during a workweek exposure. D. Henderson, R.P. Hamernik and K. Hynson (Department of Otolaryngology and Communication Sciences, State University of New York Upstate Medical Center, Syracuse, NY 13210)

Five monaural chinchillas were exposed to a repetitive and reverberant impulse noise for a period of five days, eight $\mathrm{h} /$ day at a rate of $1 \mathrm{impulse} / \mathrm{sec}$. The average peak over pressure within the holding cage was $113 \mathrm{~dB}$ SPL and the $B$ duration was $160 \mathrm{msec}$. Auditory thresholds were estimated before and after each exposure using the AER technique. Thresholds were followed for 30 days following the last exposure; the animals were then sacrificed for the surface preparation histology. A clear oscillation in the animal's threshold emerged; high frequencies $(4,8 \mathrm{kHz})$ were shifted $40 \mathrm{~dB}$ after the 8-h exposu re and recovered $25 \mathrm{~dB}$ following $16 \mathrm{~h}$ of quiet. Low frequencies $(0.25,0.5 \mathrm{kHz})$ were shifted $22 \mathrm{~dB}$ after the 8 -h exposure and recovered $25 \mathrm{~dB}$ following $16 \mathrm{~h}$ Paradoxically, the low frequencies took longer to recover from an initially lower level of TTS. In general, there was no evidence of any accumulation of TTS over the four-day period. The final audiograms will be correlated with the cochlear histology.

\section{$2: 12$}

KK4. Long-term, asymptotic threshold shift in chinchillas exposed to repetitive, reverberant, impulse noise. E.A. Blakeslee, K. Hynson, R.P. Hamernik and D. Henderson (Department of Otolaryngology and Communicative Sciences, State University of New York, Upstate Medical Center and Department of Mechanical and Aerospace Engineering, Syracuse University Syracuse, NY 13210)

Five monaural chinchillas were exposed to a repetitive, reverberant, impulse noise for ten days. The impulse-noise source was a mechanized hammer pounding on a steel plate at a rate of $1 \mathrm{impulse} / \mathrm{sec}$. The average, peak over-pressure within the holding cage was $113 \mathrm{~dB}$ SPL. Auditory thresholds were determined before and after exposu re at $0.5,1,1.4$, 2. 2.8,4,8, and $16 \mathrm{kHz}$ by means of a behavioral technique utilizing shock-avoidance conditioning. During exposure, thresholds at 0.5 and $8 \mathrm{kHz}$ were monitored on a daily basis. Within eight hours from the start of the exposure, threshold shifts at both frequencies had reached asymptotic levels of from 30 to $40 \mathrm{~dB}$. Upon removal from the noise, the threshold shift at $8 \mathrm{kHz}$ recovered more rapidly than at $0.5 \mathrm{kHz}$; however, at 40 days, both had returned to within $10 \mathrm{~dB}$ of the preexposure thresholds. A maximum PTS of $20-30 \mathrm{~dB}$ was found at either 2 or $2.8 \mathrm{kHz}$. Cochleagrams were obtained for each animal 60 days following the exposure. The histological examination provided support for the audiological findings.

\section{2:16}

KK5. Effects of noise exposure on the cochlea and neurons of the inferior colliculus. I. Melichar, J. Popelár and J. Syka (Institute of Experimental Medicine, Czechoslovak A cademy of Sciences, Prague, Czechoslovakia).

Guinea pigs were exposed for 30 or $60 \mathrm{~min}$ to high-intensity stimuli $(1 / 3$-octave band noise centered at 1 or $2 \mathrm{kHz}$ tone, intensity from 130 to $145 \mathrm{~dB}$ SPL). Several electrophysiological measurements were made in the cochlea and in the inferior colliculus before and after the exposure. Potassium concentration in the perilymph of scala vestibuli (measured with ionselective double barrel microelectrodes) which was $3.07 \pm 0.50$ $\mathrm{mm} \mathrm{K} \mathrm{K}^{+}$in the control group of nonexposed animals, increased 
after the exposure to $4.70 \pm 1.55 \mathrm{~mm} \mathrm{~K}^{+}$. Threshold tuning curves for the inferior colliculus neurons were elevated by about $30-50 \mathrm{~dB}$, when compared with the pre-exposure levels. The threshold values gradually decreased, reaching the preexposure level approximately after 48 hours. Similar time course was observed in the endochochlear potentials, which was decreased after the exposure. Despite the threshold elevation the excitability of neurons ot polarizing currents was not greatly affected by the exposure.

\section{$2: 20$}

KK6. Noise-induced threshold shifts in the Mongolian gerbil. Allen Ryan (Division of Otolaryngology, School of Medicine, University of California at San Diego, La Jolla, CA 92103)

Mongolian gerbils were trained in a shuttlebox avoidance task and their auditory thresholds determined. Groups of six subjects each were exposed to a two-octave $(1414-5656 \mathrm{~Hz})$ band of noise for one hour at intensities of 100,110, and 120 dB SPL. Thresholds between 0.1 and $16.0 \mathrm{kHz}$ were determined $0.5,3.0,6.0$, and 12.0 hours after exposure, and daily for 27 days. Final threshold determinations were made at least two months postexposure. A temporary threshold shift (TTS) was observed in all groups, the extent of which increased with increasing intensity of exposure. Recovery of thresholds followed an exponential course, and for extensive TTS Iasted throughout the 28-day observation period. In some cases additional recovery was noted upon final threshold determination. No significant permanent threshold shift (PTS) was seen in the 100-dB exposure group, but PTS did occur in the 110and $120-\mathrm{dB}$ groups. PTS was limited to a relatively narrow band of frequencies, and exhibited a half-octave shift toward higher frequencies. In terms of both TTS and PTS, the gerbil appears to be less sensitive to noise than chinchillas exposed to the same stimulus. [Work supported by NINCDS and the Medical Research Service of the Verterans Administration.]

$$
2: 24
$$

KK7. TTS in Squirrel monkeys. I. Low-frequency long duration noise. J. Burnham, D.W. Nielsen, C. Talley, and B. Deer (Otological Research Laboratory, Henry Ford Hospital, 2799 West Grand Boulevard, Detroit, MI 48202)

Little data has been gathered concerning TTS in primates following long-term exposures to noise. In this study, squirrel monkeys were exposed to an octave band noise centered at 500 $\mathrm{Hz}$ for various durations up to 48 hours. Maximum TTS occurred at 750 and $500 \mathrm{~Hz}$. An overall exposure SPL of $95 \mathrm{~dB}$ resulted in mean threshold shifts ranging from $7 \mathrm{~dB}$ for a $1-\mathrm{h}$ exposure to $20 \mathrm{~dB}$ for a $48-\mathrm{h}$ exposure. Recovery of TTS from all exposures at $95 \mathrm{~dB}$ SPL were complete within 48 hours for every subject. Comparison with the data of Melnick and Maves [Ann. Otol. 83, 820-828 (1974)] gathered on 10 human subjects indicates that both the development and recovery of TTS in squirrel monkeys is very similar to that found in humans for long-term exposures. Presently, the development and recovery of TTS to an octave-band noise centered at $4000 \mathrm{~Hz}$ is also being investigated. [This researeh supported by a Ford Foundation and an NIH grant to Henry Ford Hospital. ]

$$
2: 28
$$

KK8. Differential sensitivity to frequency and intensity in songbirds. J. M. Sinnott, M. B. Sachs, and R. D. Hienz (Johns Hopkins School of Medicine, Baltimore, MD 21205)

Frequency- and intensity-difference limens were measured in two passerine species, the red-wing blackbird (Agelaius Phoeniceus) and the brown-headed cowbird (Molothrus Ater). Operant conditioning techniques which we described previously were employed. The techniques made use of key pecks as observing and report responses. Birds were reenforced with food for reporting changes in frequency or intensity in an ongoing train of tone bursts. Smallest Weber fractions were obtained at $4 \mathrm{kHz}$ where the mean JND was $30 \mathrm{~Hz}$ or $\Delta f / f=0.0075$. The mean value of $\Delta f / f$ was 0.015 at $1 \mathrm{kHz}$ and 0.02 at $8 \mathrm{kHz}$. Birds were more sensitive to upward frequency shifts than to downward shifts at 1 and $2 \mathrm{kHz}$. Mear intensity JND's were $2.5 \mathrm{~dB}$ for a $1-\mathrm{kHz}$ tone at $50 \mathrm{~dB}$ SL cnd $5.4 \mathrm{~dB}$ for a $20-\mathrm{dB}-$ SL tone. [This work was supported by a grant from NINCIS.]

\section{$2: 32$}

KK9. Problems of guinea pig training and how to solve them. R. A. Walloch and J.A. Fenwick (Kresge Research Laboratory, University of Oregon Health Sciences Center, Portland, OR 97201)

Twenty-two guinea pigs were trained to detect a tone with a food reward. Common behavioral problems were handled in the following manner: (1) "Freezing" or fear responses were easily obviated by allowing the animals to spend a night in the training cage. (2) Initially the lever was baited to induce pressing. (3) "False positives" were punished with a 10-sec "time out," and the "false positive" rate was reduced by increasing: the number of observing responses needed to activate the tone. (4) Failures to detect the tone were also reduced by increasing the number of observing responses. (5) Despite an adequate feeding schedule, motivational levels of the quinea pigs remained a problem. [Work sponsored by the Deafness Research Foundation, Zenith Hearing Instrument Corp. and N. I. H.]

\section{$2: 36$}

KK10. Abstract withdrawn.

KK11. Auditory thresholds in the guinea pig determined by positive reinforcement methods. C.A. Prosen, M. R. Petersen, D. B. Moody, and W.C. Stebbins (Kresge Hearing Research Institute, Departments of Otorhinolaryngology and Psychology, University of Michigan Medical School, Ann A rbor, MI 48109)

Auditory thresholds of six guinea pigs were determined employing operant conditioning techniques. Each subject was trained to nose-press a transluscent response disk (the observing response) mounted on the wall of the experimental chamber just above the floor. A tone was presented through a speaker on the chamber ceiling directly over the subject's head on a variable-interval schedule. The subject reported the tone by pressing once on a second response disk (the reporting response) mounted near the first. A small pellet of lood was delivered following reporting responses made in the presence of a tone while a 15-sec time out followed false alarms. Thresholds from 125 to $45000 \mathrm{~Hz}$ were determined by the method of constant stimuli. All subjects were most sensitive to test stimuli located between 4000 and $8000 \mathrm{~Hz}$ where the threshold ranged from -10 to 0 dB re $2 \times 10^{-5} \mathrm{~N} / \mathrm{m}^{2}$. A brief training period and the lack of significant variability between or within subjects indicate that the guinea pig is a reliable species for further behavioral auditory research. The behavioral audiogram attained agreed wall with the determined 
in guinea pigs by recent investigators [Heffner, Heffner, and Masterton, J. Acoust. Soc. Am. 49, 1888-1895 (1971)] using an alternate behavioral procedure. [Supported by Research Grant NSO5077 and Program Project Grant NSO5785 from NINCDS.

$$
2: 44
$$

KK12. Apparatus and method for conditioning absolute frequency discrimination in the chinchilla. I. M. Hunter-Duvar (Department of Otolaryngology, Hospital for Sick Children, 555 University Ave., Toronto, Ontario, Canada M5G 1X8)

Absolute frequency discrimination typically has been an extremely difficult task for animals to learn. We have designed an avoidance conditioning apparatus for the chinchilla that offers the animal cues of location, shape, and color to assist it in making the correct response. The apparatus is used with a procedure which forces the animal to Iisten to the tone for several seconds before a choice can be made. Preliminary results indicate that animals can make a 2-octave discrimination at the $90 \%$ level in less than 500 trials.

$$
2: 48
$$

KK13. Auditory sensitivity of the albino rat. Jack B. Kelly and R. Bruce Masterton (Department of Psychology, Carleton University, Ottawa, Canada K1S 5B6 and Department of Psychology, Florida State University, Tallahassee, FL 32306)

Auditory sensitivity of the albino rat was determined using the conditioned suppression of licking technique. The rat was found to have a range of sensitivity to pure tones from $250 \mathrm{~Hz}$ to $80 \mathrm{kHz}$ at $70 \mathrm{~dB}$ SPL. The point of maximum sensitivity was $8 \mathrm{kHz}$ closely rivaled by higher frequencies up to $38 \mathrm{kHz}$. Withing this band, tones were detectable at sound pressures from 2-7 dB SPL. For frequencies above $38-\mathrm{kHz}$ sensitivity rapidly decreased at a rate of about $50 \mathrm{~dB}$ per octave. For frequencies below $8-\mathrm{kHz}$ sensitivity decreased at a rate of about $20 \mathrm{~dB}$ per octave. The audiogram supports previous evidence of good hearing around $32-38 \mathrm{kHz}$ [G. Gourevitch and M. H. Hack, J. Comp. Physiol. Psych. 62, 289-291 (1966)], but does not support the idea that the albino rat's hearing is narrowly tuned to this range. Thus, the sensitivity of the albino rat to these frequencies reflects the broadly tuned high frequency hearing which is generally quite good in small mammals, rather than representing a specialized feature of its auditory system. [Supported by grants from NRC and NIH.]

\section{$2: 52$}

KK14. Positive reinforcement behavioral system for tone detection and choice reaction times in the cat. J. L. Orr, D. B. Moody, and W.C. Stebbins (Kresge Hearing Research Institute and Departments of Otorhinolaryngology and Psychology, the University of Michigan, Ann A rbor, MI 48109)

The apparatus and training necessary to test cats in a tworesponse-key auditory discrimination and choice reaction time paradigm is described. Cats initiated a cycle of the discrete trial procedure by depressing two response pedals and holding them down until a light which marked the trials occurred. During trials, release of the right and left response pedals were defined as reports of tone presence and tone absence respectively. Correct release responses were reenforced with liquid food. Incorrect responses or failures to respond were not reenforced. Trials were terminated by a response or after $1.6 \mathrm{sec}$, whichever came first. Reaction times were defined as the time from trial onset until the occurrence of a response. Operation of the system was demonstrated with the method of constant stimuli. Half the trials were blanks in which no tone was presented; on the remaining trials each of 4 SPL of an 8$\mathrm{kHz}$ tone were equally likely. Detection was direct function of tone intensity and median reaction time to correctly report the presence of the tone was an inverse function of tone intensity. [Supported by Research Grant NSO5077 and Program Project Grant NSO5785 from NINCDS.]
$2: 56$

KK15. Periodicity pitch perception in cortically ablated cats. David Y. Chung (Division of Audiology and Speech Sciences, University of British Columbia, Vancouver, British Columbia, Canada V6T IW5) and Francis B. Colavita (Department of Psychology, University of Pittsburgh, Pittsburgh, PA 15213)

Six cats were first trained to discriminate frequency and shown to perceive periodicity pitch. They were then operated bilaterally with part or most of the auditory cortex aspirated out. The areas AI, AII, and Ep were ablated in two cats. They were still able to relearn frequency discrimination and perceive periodicity pitch with little amnesia. The same two cats were operated again with insular-temporal cortex (I-T) removed. Two more cats also had the areas AI, AII, Ep, and I-T removed in one operation. Two of the se four cats relearned the preoperative discrimination although considerable postoperative training was required. The other two cats showed no signs of relearning. The remaining two of the six cats had I-T lesions only. None of them acquired the preoperative discrimination. Histological examination showed incomplete ablation of the I-T areas near the rhinal fissure in the two cats which relearned the preoperative discrimination. The experiment was unable to resolve the cortical areas for the pure-tone and the periodicity-pitch discrimination. The fact that both kinds of discrimination occurred together may imply that the cortex is not an essential part of the auditory system for either kind of discrimination.

\section{$3: 00$}

KK16. Hearing in large and small dogs (Canis familiaris). H. Heffner (Bureau of Child Research, University of Kansas located at Parsons State Hospital, Parsons, KS 67357)

Audiograms were determined for four dogs ranging in size from 4.5 to $30 \mathrm{~kg}$. The dogs were tested using a two-choice procedure with a ready response, i.e., the dogs initiated a trial by licking a center water spout and were rewarded with water for licking a left water spout if a tone was present or for licking a right water spout if no tone was present. At a level of $60 \mathrm{~dB}$ SPL, the dogs were able to hear as low as 62 $\mathrm{Hz}$ and as high as $45 \mathrm{kHz}$ with a best frequency of $8 \mathrm{kHz}$. In spite of the large variation in head size, there appeared to be no difference between the ability of large and small dogs to hear high frequencies. Thus it appears that the inverse relationship between the functional distance between the two ears and high-frequency hearing may not apply to variation within a species. [Supported by NIH grants NS 12992 and HD 02528.]

\section{3:04}

KK17. Discrimination of pure-tone intensities by the California sea lion. P.W.B. Moore and R.J. Schusterman (Departments of Psychology and Biology, California State University, Hayward, CA 94542)

The ability of the California sea lion (Zalophus californianus) to detect intensity differences of $16-\mathrm{kHz}$ pure-tone pulses was determined in a yes-no signal detection task with trial by trial feedback, using a modified method of limits similar to the "staircase method." Additionally, three prethreshold experiments were performed to determine the effect of stimulus presentation strategy on discrimination ability. Results of the prethreshold experiments suggest that a "collapse" method (i. e., symmetrically reducing the intensity of the louder tone pulse in a pair and increasing the intensity of the softer) resulted in smaller threshold estimates than either an ascending or descending staircase method. Under the collapse method, a difference threshold estimate of $3.19 \mathrm{~dB}$ was found for 16$\mathrm{kHz}$ pure-tone pulses. These results support a previous hypothesis based of behavioral underwater sound localization experiments and sound-skull measurements by Moore and $\mathrm{Au}$ [J. A coust. Soc. Am. 58, 721-727 (1975)]. These experiments also indicated that a sea lion which persistently biased its responses by making a low rate of false alarms in an absolute threshold task [Schusterman, J. A coust. Soc. Am. 55, 
$845-848$ (1974)] did not bias its responses in al difference threshold task.

\section{$3: 08$}

KK18. Partial masking effect in the monkey as measured by a behavioral technique. D. B. Moody, W.C. Stebbins, and $\bar{M}$. C. Rudy (Kresge Hearing Research Institute and the Departments of Otorhinolaryngology and Psychology, University of Michigan Medical School, Ann Arbor, MI 48109)

Macaque monkeys were trained with a positive-reenforcement behavioral procedure to release a response key when a tone was presented. The latency of this release reponse is an inverse function of the sound pressure of the tone over a wide dynamic range, and can be used as a behavioral index of relative louchess. When tested on this procedure in the presence of moderate levels of masking noise, the monkeys showed the greatest effect of the masker for low levels of test tone while the masker became less effective with more intense test tones. These data thus mirror the partial masking phenomenon described for human subjects and are similar to loudness recruitment data previously demonstrated in humans and monkeys. When maskers of different bandwidths were equated for amount of threshold shift, no consistent difference between the bandwidths in effectiveness at higher test-tone levels was observed; that is, the more rapid growth of loudness associated with narrower band maskers which has been reported in humans was not observed in all subjects. [Supported by Research Grant NSO5077 and Program Project Grant NSO5785 from NINCDS. ]

$$
3: 12
$$

KK19. Perception of conspecific communication signals by Japanese monkeys (Macaca fuscata). M. D. Beecher, S. R. Zoloth, M. R. Petersen, D. B. Moody, and W.C. Stebbins (Kresge Hearing Research Institute, University of Michigan, Ann A rbor, MI, and Center for Field Research, Rockefeller University, Millbrook, NY)

A method for assessing perception by monkeys of their own acoustic commmunication signals is described. Japanese macaques were trained with a positive-reenforcement conditioning procedure to discriminate between two classes of conspecific vocalizations, "smooth early highs" (SEH) and "smooth late highs" (SLH) [classification of Green, Primate Behavior 4, 1-101 (1975)]. Subjects contacted a response disk to initiate a series of stimuli from the SLH class. A trial was defined as an insertion of a SEH stimulus into the series in place of a SLH. A key release on such a trial (correct detection) was reenforced with food. With this method we are examining a variety of perceptual questions regarding the animal's acoustic communication system. For example, do the animals in the laboratory task classify these calls as they do in the field? Do they perceive acoustic intermediates as true intermediates or as members of one or the other signal class in the manner that humans categorize their own speech sounds. [Supported by
NSF Research Grants BMS 74-20050 and 5-27092, NINCDS Program Project Grant NSO5785, and NIGMS Training Grant GM-01789. ]

$$
3: 16
$$

KK20. Primate auditory localization: the locatability of different bandwidth signals. C.H. Brown, M. D. Beecher, D. B. Moody, and W.C. Stebbins (Kresge Hearing Research Institute, University of Michigan Medical Center, Ann Arbor, MI 48109)

The ability of monkeys (Macaca) to detect a change in the location of a sound in space was psychophysically assessed through the employment of operant-conditioning techniques. Contact with a response disk initiated a train of acoustic stimuli pulsed from a standard location $\left(0^{\circ}\right.$ azimuth). Following a variable number of pulses the stimulus changed its spatial position to one of several comparison locations. If the monkey reported this change in location by releasing the response disk it received food for reenforcement. Thresholds for the minimum discriminable change in the hor zontal coordinate of sound in space (azimuth) were determined by the method of constant stimuli under free-field conditions in an anechoic chamber. The monkeys were tested with a parametric array of pure tones and signals of different bandwidths. The results indicate that the inverse relationship between threshold and stimulus bandwidth found in a preliminary study IC. H. Brown, M. D. Beecher, D. B. Moody, and W. C. Stebbins, J. Acoust. Soc. Am. 58, 124S (1975)] characterizes Nacaca localization over much of its range of hearing. [This research was supported by NSF Grant No. BMS74-20050 and Program Project Grant NSO5785.]

$$
3: 20
$$

KK21. Interaural time-difference threshold in pig-tailed monkeys (M. nemestrina). D. Houben and G. Gourevitch (Department of Psychology, Hunter College, CUNY, New York, NY 10021)

A brief $60 \mathrm{~dB}$ SPL diotic tone burst was delivered to the monkey, followed by a dichotic burst of interaurally delayed tones. The monkey was trained to respond on a right lever when the phase of the dichotic burst was leading at the right ear, and on a left lever when the phase was leading at the left ear. The side of the leading ear and the magnitude of the interaural delay were chosen at random from trial to trial. Interaural time delay (ITD) threshold was taken as the time disparity correctly lateralized on $75 \%$ of the trials and was measured at eight frequencies ranging from $250 \mathrm{~Hz}_{z}$ to $2 \mathrm{kHz}$. The ITD funetion decreases from 250 to $750 \mathrm{~Hz}$; thereafter it remains constant at about $50 \mu \mathrm{sec}$. This function parallels the human curve, [J. Zwislocki and R.S. Feldman, J. Acoust. Soc. Am. 28, $860-864$ (1956)] although it is elevated by about $30 \mu \mathrm{sec}$. Humans cannot utilize ongoing time differences to lateralize pure tones above $1300 \mathrm{~Hz}$, whereas the monkeys were able to lateralize up to $2000 \mathrm{~Hz}$. The importance of ITD's in monkey auditory localization is discussed. 


\title{
Session LL. Speech Communication VI: Perception I
}

\author{
Michael Studdert-Kennedy, Chairperson \\ Department of Communication Arts and Sciences, Queens College, Flushing, New York 11367
}

and

\author{
Haskins Laboratories, New Haven, Connecticut 06510
}

\section{Contributed Papers}

2:00

LL1. Stop bursts and transitions as functionally equivalent, context-sensitive cues. M.F. Dorman, * Michael StuddertKennedy, ${ }^{\dagger}$ and Lawrence J. Raphael, ${ }^{\ddagger}$ (Haskins Laboratories, New Haven, CT 06510)

The experiment reported here was primarily designed to test the functional invariance of stop bursts as perceptual cues. Bursts from three sets of nine CV syllables for each stopconsonant place of articulation were transposed within each set and played for listeners. Although some measure of perceptual invariance was found within each of the labial and apical sets, and within two subsets for the velar bursts, the perceptual weight carried by the bursts attained significance in few of the syllables tested. Further, the perceptual weight of the bursts and formant transitions of the syllables tended to be reciprocally related. These two cues appear to be functionally equivalent, context-dependent cues, each contributing to the rapid spectral changes that follow consonantal release. The results indicate the importance of the role of front cavity resonance in signaling place of articulation.

*Also at A rizona State University, Tempe, AZ 85281.

${ }^{\dagger}$ Also at Queens College, CUNY, New York, NY 10031.

†Also at Herbert H. Lehman College, CUNY, New York, NY 10031.

$$
2: 10
$$

LL2. Perceptual invariance and onset spectra for stop consonants in different vowel environments. S. E. Blumstein (Department of Linguistics, Brown University, Providence, RI 02912) and K. N. Stevens (Research Laboratory of Electronics, Massachusetts Institute of Technology, Cambridge, MA 02139)

In this series of perception experiments, we have attempted (a) to determine if a brief stimulus in which only the spectral information at on set is preserved provides sufficient cues for identification of place of articulation across vowel contexts, and (b) if it does, to define further the nature and size of the spectral window. Subjects were randomly presented with synthetically produced stimuli consisting of a 5- or $10-\mathrm{msec}$ noise burst followed by a brief voiced interval containing three formant transitions with onset and off set characteristics appropriate to the consonants $[b, d, g]$ in the environment of the vowels $[a, i, u]$, as well as stimuli with steady second- and third-formant transitions. The length of the voiced interval was systematically varied from 40 to $5 \mathrm{msec}$. The results indicate that an onset spectrum consisting of the burst plus the initial 5 or $10 \mathrm{msec}$ of voicing provide sufficient cues for the identification of the stop consonant, and that vocalic information can be reliably derived from these brief stimuli containing only one or two glottal pulses. [Research approved by an NIH grant.]

$$
2: 20
$$

LL3. Secondary acoustic characteristics and vowel identification. D. M. Bischoff (Department of Hearing and Speech Sciences, Stanford University, Stanford, CA 94305)
The relation between primary acoustic cues and secondary acoustic characteristies of rowels was investigated by asking subjects to identify vowels in different contexts and under different conditions. Six i solated rowels, $/ \mathrm{a} / \mathrm{i} / \mathrm{i} / \mathrm{u} /, / \mathrm{e} /$, $/ \mathrm{I} /$, and $/ \epsilon /$ were presented under nine conditions: naturally spoken, synthesized, synthesized with no differential fundamental frequency information, synthesized with no differential durational information, synthesized with no formant structure, synthesized with no differential fundamental frequency or durational information, synthesized with neither format structure nor differential fundamental frequency information, synthesized with neither formant structure nor differential durational information, and synthesized with no formant structure and no differential fundamental frequency or durational informational information, synthesized with neither formant structure contexts and presented to subjects for identification. These contexts were chosen to either minimize or maximize the consonant-vowel transitional information. The results will be discussed in terms of the relative importance of primary and secondary acoustic characteristics for vowel identification.

\section{$2: 30$}

LL4. Perception of synthetic unsteady vowels under various formant conditions. Hisao Kuwahara and Hisao Sakai (Broadcasting Science Research Laboratories of Japan Broadcasting Corporation, Tokyo 157, Japan)

Aural tests were done for stimuli consisting of three-vow'el chain synthesized by means of terminal-analog speech synthesizer. The second-formant frequency corresponding to the middle vowel of the stimuli changed along a stimulus continuum from $/ \mathrm{u} /$ to $/ \mathrm{i} /$, while other formants were held constant. The initial and final vowels of the stimuli were the same vowel $/ \mathrm{u} /$, and the duration and frequency of the second formant corresponding to the middle vowel had different values. The formant transition changed linearly with different rates. Subjects identified the middle vowel of each stimulus as one of Japanese vowels $/ \mathrm{u} /$ or $/ \mathrm{i} /$. The results indicated that the changed rate of the formant transition and the duration of the midpart of steady state had little effect on vowel perception if the whole duration between the initial and final vowels became longer than $50 \mathrm{msec}$.

$$
2: 40
$$

LL5. Speech perception in early infancy: Perceptual constancy for vowel categories. Patricia K. Kuhl (Child Language Acquisition Project, University of Washington, Seattle, WA 98195)

The young infant discriminates a single token of /a/ from a single token of $/ i /$ and can successfully ignore variations in the pitch contour of these vowels [Kuhl and Miller, J. A coust. Soc. Am. 59, S54(A) (1976)], but demonstrations that an infant perceives an inherent similarity in a male's /a/ and a female's /a/ are lacking. Three infants, $5 \frac{1}{2}$ - to 6 -months old, were tested in a task in which a bead-turn response was reinforced with a visual stimulus in the presence of a speech sound from 
one category but not in the presence of a speech sound from a second category. The infants were trained with a single synthetically produced / $a$ / and / $i$ / whose formant frequencies and fundamental frequency were appropriate for a male voice (rise-fall pitch contour) and then tested with synthetic/a/ and $/ i /$ tokens whose formant frequencies and fundamental frequencies were appropriate for female and child talkers, with both rising and rise-fall pitch contours. Generalization to novel tokens was often times immediate. The six-month old's behavior is suggestive of either an inherent, or easily learned, perceptual constancy for vowel cat egories when both the critical (formant frequencies) and noncritical (fundamental frequency, pitch contour) acoustic dimensions are randomly varied.

$$
2: 50
$$

LL6. Discrimination of synthetic prevoiced labial stops by infants and adults. Rebecca E. Eilers (Mailman Center for Child Development, P.O. Box 520006, Biscayne Annex, Miami, FL 33152), Wesley R. Wilson and John M. Moore (Department of Speech, University of Washington, Seattle, WA 98195)

Individual data were obtained from eight normal 6-monthold infants on discrimination of eight synthetic labial stop pairs differentiable on the basis of voice onset time. All infants were presented with the pairs $p^{h_{a}}(+40)$ versus $p^{h} a(+70)$, $p^{\text {ha }}(+40)$ versus pa $(+10)$, pa $(+10)$ versus ba $(-20)$, ba $(-20)$ versus ba $(-50)$, ba $(-20)$ versus ba $(-60)$, ba $(-30)$ versus ba $(-60)$, pa $(0)$ versus ba $(-30)$ and pa $(+10)$ versus ba $(-60)$. Discrimination was assessed for each infant on each pair using the VRISD paradigm (Visually Reinforced Infant Speech Discrimination). AduIt discrimination was assessed for the same pairs in the same manner. With few exceptions, infants presented no evidence of discrimination of pairs other than the pair $\mathrm{p}^{\mathrm{h}} \mathrm{a}(+40)$ versus pa $(+10)$ despite the fact that these infants were successful in the same paradigm with a variety of subtle natural speech contrasts. Contrary to the hypothesis that age restricts discrimination capacities, adults were far more successful than infants in discriminating the non-English contrasts. Individual data will be presented to illustrate these points and the use of synthetic speech stimuli in infant research will be discussed in light of these data. (Works supported by NIH-NICHD, HD-3-2793.]

\section{$3: 00$}

LL7. Stimulus correlates in the perception of voice onset time (VOT): I. Discrimination of the time interval between tone bursts of different intensities and frequencies. P. L. Divenyi, * R. M. Sachs, and K. W. Grant (Central Institute for the Deaf, $818 \mathrm{~S}$. Euclid, St. Louis, MO 63110)

In a four-level $2 \mathrm{AFC}$ paradigm, trained subjects had to discriminate time intervals that were marked by tone bursts of unequal duration (10 and $100 \mathrm{msec}$ ). Time differences discriminable at the $d^{\prime}=1$ level, $\Delta T_{\text {Theal }}$ were determined in the $10-100-\mathrm{msec}$ range of time intervals (onset to onset). When the frequencies and intensities of all markers were held constant ( $1 \mathrm{kHz}$ and $86 \mathrm{~dB}$ SPL), $\Delta T_{\mathrm{T} \text { mesh }}$ was a monotonic function of $T$. This relation became non-monotonic when the level of the first marker was decreased to $36 \mathrm{~dB}$ SPL or as the two marker frequencies were made different from each other by two octaves. Thus, a discrepancy in either the level or the spectrum of two time-marker sounds may constitute an acoustic basis for the nonmonotonic VOT discrimination functions, despite the fact that the depth of the local minimum found in the present data is less than that observed in VOT experiments. [Supported by a grant from NINCDS.]

*Presently at Neurophysiology-Biophysics Laboratories, Veterans Administration Hospital, Martinez, CA 94553.

$$
3: 10
$$

LL8. Stimulus correlates in the perception of voice onset time (VOT): II. Discrimination of speech with high and low stimulus uncertainty. R.M. Sachs and K.W. Grant (Central Institute for the Deaf, $818 \mathrm{~S}$. Euclid, St. Louis, MO 63110)
The three Lisker-Abramson CV series (ba-pa, da-ta, ga-ka) were computer synthesized at C.I. D. in 1-msec steps. For each 84-trial block of a high-uncertainty, same-different task (no feedback), one of the three continua was chosen at random. On each trial, the ability of three naive listeners to resolve for differences among VOT pairs ranging from VOT $=10-80 \mathrm{msec}$, in 10-m sec steps was tested. A s expected, there was a local maximum performance for each of the three speech continua, thus satisfying one criterion for categorical perception. A minimum-uncertainty A.X task (with feedback) was run with three highly trained subjects for the ga-ka continuum. Only one standard VOT was Fresented in a block of trials. Results were not categorical: the minimum detectable $\triangle V O T$ was less than $2 \mathrm{msec}$ at $V O T=10 \mathrm{msec}$ and increased as VOT increased. Our results indicate that trained listeners can utilize subtle stimulus cues in syrthetic speech if stimulus uncertainty is minimized. [Supported by a grant from NINCDS.]

\section{$3: 20$}

LL9. The voicing boundary as a function of $F_{2}$ and $F_{3}$ transitions and fundamental frequency. Brumo $\mathrm{H}$. Repp (Haskins Laboratories, 270 Crown St., New Haven, CT 06510)

It is well known that the voice-onset-time (VOT) boundary for synthetic stop consonants shifts towards longer VOT's as place of articulation shifts from front to back, even if all parameters of the signal except VOT and the starting frequencies of $F_{2}$ and $F_{3}$ are held constant. Is this a phonetic effect contingent on a decision about the place feature, or is it an auditory effect that is somehow dependent on the spectrum at syllable onset? In order to decide thici question, $F_{2}$ and $F_{3}$ transitions were varied in a number of steps along a "place continuum," and the VOT boundary wass determined at each step. The function relating the VOT boundary to the place continuum should be flat within place categories and show jumps at the category boundaries if the effect is phonetic, but. it should be monotonically increasing if the effect is auditory. Neither hypothesis has been supported by the data so far, which show the function to be generally increasing but strongly nonmonotonic, with a number of seerringly haphazard but statistically reliable peaks and valley;. A radically different pattern (with the same general characteristics) is obtained if the fundamental frequency of the stimuli is changed. Further research on the acoustic basis of the effect and its perceptual. and methodological implications will ke reported.

$$
3: 30
$$

LL10. Perception of voicing in final stops. Catherine G. Wolf (36-549, Massachusetts Institute of Technology, Cambridge, MA 02139)

The perception of voicing in final stops was investigated. Syllables which had been truncated at various points were presented to subjects for identification under two response conditions. In one condition, the response set consisted of two consonants (one voiced, one voicelessl. In the other condition, subjects could choose either of the two consonants, or report that no final consonant was heard. The results indicated that the formant transitions, closure, burst, and vowel duration are important in determining whether a stimulus is heard as voiced or voiceless. In addition, there are coarticulation effects occurring as early as the first $50 \mathrm{msec}$ of the preceding vowel which appear to affect the pattesn of voiced/voiceless judgments. A common basis for the perception of voicing in initial and final stops is also considered. (Work supported by NINCDS. ]

$$
3: 30
$$

LL11. Vowel duration as a cue for consonant voicing? C.W. Fruin and D. M. Bischoff (Department of Hearing and Speech Sciences, Stanford University, Stanford, CA 94305)

Several studies state that the duration of the vowel preceding the consonant is a significant cue to the voicing characteristic of that consonant. The CVC's used in these studies have generally been prepared on the Pattern Playback. The present 
study was designed to examine vowel duration as a consonant voicing cue in naturally produced speech. Several CVC's were recorded by both authors as stressed citation forms. Vowel durations were measured and parts were removed from: the endmost section of the vowel, the middle of the vowel, or the end and the middle of the vowel. At least for these stimuli produced by these speakers, no clear relation is seen between the length of the preceding vowel and the perception of the voicing characteristic of the final consonant.

\section{$3: 50$}

LL12. Perception of vowel duration. William S-Y. Wang (Project on Linguistic Analysis, 2222 Piedmont Ave., University of California, Berkeley, CA 94720), Ilse Lehiste (Department of Linguistics, Ohio State University, Columbus, $\mathrm{OH}$ 43210), Chin-Kuang Chuang, and Nancy Darnovsky (Project on Linguistic Analysis, 2222 Piedmont Ave., University of California, Berkeley, CA 94720)

Lehiste and Pisoni have independently shown that the perception of vowel duration is influenced by the $F_{0}$ pattern that accompanies the vowel. Here we report some related results. Sixteen subjects are asked to judge the relative duration of pairs of synthesized stimuli. The stimulus pairs are made up of the vowels $/ i /, / a /, / a^{i} /$, and nonspeech, where nonspeech is a single formant at $1500 \mathrm{~Hz}$. The $F_{0}$ patterns are: level $=120 \mathrm{~Hz}$, rising $=105-135 \mathrm{~Hz}$, and falling $=135$ to $105 \mathrm{~Hz}$. The durations are $220,240,260$, and $280 \mathrm{msec}$. The orthogonal combination of these factors makes up a total of 512 stimulus pairs. In comparing duration judgements of stimuli of rising (R), falling (F), and level (L) $F_{0}{ }^{\prime} s$, we found that the following relation obtains: $R>F>L$, where " $>$ " denotes "perceived as having greater duration." This relation holds to the same extent for both speech and nonspeech stimuli. There is no signficant difference in the perceived duration between $/ i /$ and $/ a^{i} /$. The most consistent effect, found also earlier in a pilot experiment we performed with 58 subjects, is that $/ i /$ is heard as longer than $/ a /$, in a ratio of $60 \%: 40 \%$. Since / $/$ / is normally shorter than /a/ in natural speech, we posit a compensation mechanism mediating between perception and production that is responsible for this negative correlation between vowel height and duration. It is interesting to note that although the processes of duration judgment and pitch judgement (cf. the abstract by Chuang and Wang) are different, they seem to share a common compensation mechanism between production and perception. [Work supported by NSF Grant No. BNS 76-00017-Wang-2/78J34.2.]

\section{$4: 00$}

LL13. Influence of vowel height, intensity, and temporal order on pitch perception. Chiu-Kuang Chuang and William S-Y. Wang (Project on Linguistic Analysis, 2222 Piedmont Ave., University of California, Berkeley, CA 94720)

Recently, it has been observed that high vowels are produced with a higher $F_{0}$, even in tone languages. Earlier, $\mathrm{H}$ Fletcher reported that louder pure tones are perceived as having a lower pitch, but only in the range 50 to $500 \mathrm{~Hz}$, which is the range of $F_{0}$ in speech. Here we report some experiments which investigate these relations. Fifteen subjects are asked to judge the relative pitch of pairs of synthesized vowels. The vowels are $/ \mathrm{i}, \mathrm{e}, \mathrm{u}, \mathrm{a} / \mathrm{with}$ intensity differences $(\Delta I)$ of 0,10 , 20 , and $30 \mathrm{~dB}$ SPL, and at frequency differences $\left(\Delta F_{0}\right)$ of $0,1,2$, and $3 \mathrm{~Hz}$, with a base frequency of $100 \mathrm{~Hz}$. Each vowel is $300 \mathrm{msec}$ long and the inter stimulus interval is $400 \mathrm{msec}$. The orthogonal combination of these factors, i. e., four vowel types, four $\Delta \gamma^{\prime} s$, four $\Delta F_{0}^{\prime} s$, and the two positions within each pair, makes up a total of 512 stimulus pairs. The interaction between pitch perception and vowel height is highly significant statistically. Pooled across all subjects, /a/ is heard as $2.2 \mathrm{~Hz}$ higher than $/ \mathrm{u} /, 0.8 \mathrm{~Hz}$ higher than $/ \mathrm{i} /$, and 0.2 $\mathrm{Hz}$ higher than /e/. The intensity factor by itself appears to have no effect on pitch perception, but interacts with the postion factor in influencing pitch perception. The negative correlation between production and perception in the relation between vowel height and pitch is interpreted as the result of a compensation mechanism mediating between production and perception. [Work supported by NSF Grant No. BNS 76-00017Wang-2/78J34.2. I

\section{$4: 10$}

LL14. An acoustic correlate of syllabicity in english.

H. Semiloff (Speech Communications Research Laboratory, Santa Barbara, CA 93109)

The purpose of this research was to test three hypotheses (1) Increasing the duration of a nonsyllabic segment will cause the segment to be perceived as syllabic. (2) The perception of syllabicity is categorical. (3) Listeners will differ in their labeling responses to stimuli when told to judge them in different speech styles. Seven words were synthesized: "blow," "plight," "dress," "crest," "prayed," "broke," and "sport." The steady-state portion of the $/ 1 /$ or the $/ r$ / (for "sport," aspiration of $/ \mathrm{p} /$ ) was lengthened in $10-\mathrm{msec}$ increments. Subjects were asked to decide whether the word was monosyllabic or disyllabic: "blow-below," "plight-polite," "dress-duress," "crest-caressed," "prayed-parade," "broke-baroque," and "sport-support." Five groups of 15 listeners each participated in the labeling task. Four groups heard the stimuli with a precursor frame, "The word you will hear next is _" in one of four speech styles: formal-slow, formal-fast, casual-slow, casual-fast. Each group was told to judge the words according to the criterion of being spoken in one of the designated styles. The fifth group heard the stimuli with no precursor and received no style instructions. Results showed that (1) increased durations of $/ r /$ and $/ 1 /$ did result in perception of the words as disyllabic, but increasing the duration of aspiration in "sport" did not; (2) perception of syllabicity appears to be categorical; and (3) there were no statistical differences among the four speech styles. [Work supported by NSF SOC 75-10043.]

\section{$4: 20$}

LL15. Stimulus range as a determinant of phoneme boundaries along synthetic consonant continua. Michael Studdert-Kennedy (Queens College, CUNY, Flushing, NY 11367 and Haskins Laboratories, 270 C rown St., New Haven, CT 06510)

Brady and Darwin (see Darwin, C.J. The Perception of Speech, in E.C. Carterette, and M. P. Friedman, Handbook of Perception, Academic, New York (in press), Vol. 7, report shifts in the phoneme boundary along a synthetic voicing continuum as a function of the range of stimuli presented within a test. The present study reports comparable shifts along synthetic voicing and place of articulation continua. Implications for the interpretation of adaptation boundary shifts are considered.

$$
4: 30
$$

LL16. Perceptual adaptation to the duration of vowels preceding stop consonants. Paul D. Williams and Donald J. Sharf (Section of Speech and Hearing Sciences, The University of Michigan, 1111 E. Catherine St., Ann A rbor, MI 48104)

The affect of selective adaptation on the ability to identify stop consonants as being either voiced or voiceless solely on the basis of preceding vowel duration was tested. Stimuli consisted of computer-modified, real-speech sounds edited from a single utterance of /ad/ which varied in duration in 25-msec steps. Each subject classified recorded, random orderings of the stimuli as either AT or AD before and after periods of repetitive listening to long and short adapting stimuli. Calculated phonetic boundaries showed a mean shift toward the category of the adapting stimulus in both conditions, indicating a reduction in sensitivity for that category. The findings of independent, adaptable feature detectors for the voiced and voiceless categories and a greater resistance to adaptation for the voiced feature were comparable to results reported for adaptation studies employing voice on set time. [P.D. Eimas and J. D. Corbit, Cognitive Psychology 4, 99-109 (1973); P. D. Eimas et al., Perception and Psychophysics 13, $247-253$ (1973)]. 
LL17. Abstract withdrawn.

\section{4: 50}

LL18. Categorization of nonspeech signals. Karen Forrest (A coustics Research Department, Bell Laboratories, Murray Hill, NJ 07974)

Investigations of speech perception have demonstrated the existence of discrete perceptual categories when stimuli vary along a continuous frequency dimension. Nonspeech signals that vary along a continuous frequency dimension, when interspersed with speech signals, are not perceived in a categorical manner. This has led to the assumption that speech is an acoustic signal with properties that are different from nonspeech acoustic signals. The present study was designed to determine the validity of this distinction as it relates to categorization. The stimuli used were complex tones composed of a constant $800-\mathrm{Hz}$ tone and a glide with a variable starting point and a fixed endpoint at $2 \mathrm{kHz}$. The rate of frequency change was also varied. All stimuli were $30 \mathrm{msec}$ in duration. The stimuli were separated into two groups-Group A stimuli in- creased to $2 \mathrm{kHz}$, and Group B decreased to $2 \mathrm{kHz}$. Subjects were required to group the stimuli in:o appropriate categories. They received no information about what stimulus belonged to what category, but feedback was provided after each trial. Initial data show that subjects were alole to categorize the stimuli with a high degree of accuracy after a rather limited period of exposure.

$$
\text { 5:00 }
$$

LL19. Discrimination-level differences (DLD's) as a measure of the uniqueness of speech. Jerry $V$. Tobias (Civil Aeromedical Institute, Federal A viation Administration, Oklahoma City, OK 73125) and Gerald D. Kidd, Jr. (University of Oklahoma Health Science Center, Oklahomal City, OK 73190)

The analysis of speech signals is oiten reported to be different from the analysis of nonspeech signals. From the premise that this difference is characteristic not of the speech/ nonspeech nature of a sound, but of a meaningful/meaningless dichotomy, binaural masking tests were made on a hierarchy of six computer-generated approximations to speech, all with similar temporal patterns; they ranged from a nonsense syllable through a shaped noise and a tone burst. Two kinds of tests were run, one at and one above threshold. The at-threshold test was a simple masking-level-difference measurement that compared homophasic $\left(N_{0} S_{0}\right)$ with antiphasic $\left(N_{0} S_{n}\right)$ detection of each signal. The above-threshold test was comparable to an intelligibility-level-difference measurement (al so comparing $N_{0} S_{0}$ with $N_{0} S_{\pi}$ presentations); however, subjects were asked to discriminate the six types of sound rather than to recognize words. The result is a discrimination-level difference (DLD). In preparation for this identification experiment, listeners learned to discriminate the signals in the quiet and at favorable signal-to-noise ratios; practice continued long past the time when they could make $100 \%$ correct judgments. A pparently as a result of this overtraining, subjects gave DLD's for the speech signals that differ little if at all from the DLD's for the nonspeech signals - even for those at the other extreme of the hierarchy. This finding suggests that once a sound is identified as having a special communicative function, it is specially treated by the auditory system, much as many of us have always assumed that speech is specially treated. Additional data will be presented comparing detection perfor-mance with identification performance.

\title{
Session MM. Musical Acoustics II: Music Perception and Memory
}

\author{
W. Savage, Chairperson \\ University of Iowa, Iowa City, Iowa 52242
}

Invited Papers

2:00

MM1. How a composer looks at organization. Joel Chadabe (Electronic Music Studio, State University of New York at Albany, Albany, NY 12222)

Two discrete levels represented one after another on a graph are normally considere $\mathrm{t}$ to be connected by a vertical line which has no time value. But the realities of engineering teach us that any vertical line has a time value, no matter how short. If that graph is charting the sound from a single source, we may observe that even extremely dissimilar sounds are con- 
nected in a continuum; that they are not items that are arranged, but rather a continuous process of change. Changes may be periodic, transient or random, and they exist at different time scales from "audiotime" (changes in milleseconds) to "conscious time" (changes in seconds). These changes attribute to a sound's "meaning." The problem for a composer of computer music is how to generate these changes meaningfully.

MM2. Interaction between rhythmic prominences and intonation. Paul C. Boomsliter (State University of New York at Albany, NY 12222) and Warren Creel (Albany Medical College, Albany, NY 12208)

Students played scales on equipment which provided altermative tunings of scale notes. When instructed to dwell on one or another note, they chose different tunings for the subsequent notes of the scale. This suggests that when a note is made prominent, it is likely to establish a second tonal center to which later intonations are related. Thus the rhythmic structure of a melodic phrase interacts with pitch choices in performance.

MM3. Octave equivalence and the processing of melodic sequences. Diana Deutsch (Center for Human Information Processing, University of California at San Diego, La Jolla, CA 92093)

It is clear that a certain perceptual equivalence exists between tones standing in an octave relationship. However, it was previously found that subjects were unable to recognize a wellknown melody when its component tones were placed randomly in any one of three octaves. In the present experiment, subjects listened to a standard melody, and then to a comparison melody; and they judged whether the comparison melody was an exact transposition of the standard or not. Exact repetition of the standard melody produced a significant improvement in performance, which also occurred when the repeated melody was displaced entirely to the octave above or the octave below that of the standard. However, when the melody was repeated such that its individual components were placed alternately in the octave above and the octave below, performance was significantly poorer than when the melody was not repeated at all. It appears that octave equivalence does not operate in short term melody acquisition any more than in long term melody recognition.

$$
3: 15
$$

MM4. Balancing the scales for music and speech. E. M. Burns, A. E. Carney, and W. D. Ward (Hearing Research Laboratory, University of Minnesota, 2630 University Ave. S. E. Minneapolis, MN 55414)

The results of our experiments on the identification and discrimination of musical intervals, along with the results of experiments by other investigators, are interpreted with regard to the possible "natural" basis of musical scales. We have concluded, primarily from basic differences in the perception of intervals by musicians and nonmusicians, that there is little evidence for the existence of "natural" interval categories, either in the sense of being innately determined by characteristics of the auditory processing system, or in the sense of early learning of the relations between partials in natural sounds. In light of the fact that many of the characteristics associated with the perception of speech, e.g., robust categorical perception, adaptation, and dichotic ear advantage, are also evident in the perception of musical intervals, the general question of the relationship between music perception and speech perception is also discussed.

MM5. Formation of auditory descriptions. A.S. Bregman (Department of Psychology, McGill University, Montreal, Quebec H3C 3G1)

In natural environments, sounds from many sources are mixed at the ear. The auditory system must parse the input to recover separate descriptions of the original sources. In addition to mechanisms for sound localization, it employs "compositional heuristics" for segregating and combining acoustic components to create a perceptual description of simultaneously active acoustic sources. The clues in the acoustic signal that indicate that components should be assigned to a single source include spectral similarity of successive components, slow and continuous changes, information for "temporary occlusion," and, finally, synchronous and parallel changes of simultaneous components. Descriptions of multiple sources are built up simultaneously, and these descriptions compete for acoustic elements until elements are assigned as belonging to specific sources. The perception of certain qualitative features, such as timbre, depend on the perceptual grouping of components. Other perceptual consequences, such as the number of sources perceived, and the ability to judge the temporal order of elements, or to recognize patterns, also depend upon the preliminary parsing of the signal as controlled by the "compositional heuristics." To demonstrate these effects, experiments will be described and recorded stimuli.will be played.

$$
4: 05
$$

MM6. Perceptual space of musical structures. E. C. Carterette (Department of Psychology, University of California, Los Angeles, CA 90024)

Loudness or pitch can be scaled in a space of one dirnension. Timbre is multidimensional. 
The timbre of a musical sound or voice depends upon its time-varying spectral envelcpe and periodic fluctuations of amplitude or fundamental frequency. The strengths and weaknesses of scales in describing aspects of tone sensation are contrasted in the light of experiment and theory, both old and new. Multidimensional examples are perceptual spaces of voices and musical structures. Unidimensional examples are the standard scales of loudness and pitch. The adequacy of these is questioned by some recent work done with Norman H. Anderson and based on functional measurement theory. Thus, in one series of experiments, subjects judged loudness averages and differences of noise arrays. The resulting interval scala indicates that loudness is the one-third power of sound pressure. New work on loudness and pitch scales obtained in bisectioning tasks will be presented.

\title{
Panel Discussion
}

$$
4: 30
$$

MM7. Panel Discussion.

\section{Panel Members:}

F. Altneave

Department of Psychology, University of Oregon, Eugene, OR 97403

I. Pollack Mental Health Research Institute, University of Michigan, Ann Arbor, MI 48104

\section{Session NN. Underwater Acoustics VI: Seismic Profiling}

\author{
Ira Dyer, Chairperson \\ Ocean Engineering Department, Massachusetts Institute of Technology, \\ Cambridge, Massachusetts 02139
}

\section{Invited Papers}

$2: 00$

NN1. Migration of reflection seismic data: Two approaches. K. L. Larner and L. Hatton (Western Geophysical Company, P.O. Box 2469, Houston, TX 77001)

A conventional reflection seismic record section displays data only as passing wavefield recorded at points on the earth's surface. In regions of complex geology, this display may bear little resemblance to a cross-section of subsurface reflectors. Migration is the technique used to transform such a wavefield into a reflectivity display. Different migration methods are derived from either an integral or differential form of the scalar wave equation. In particular, a matched filtering technique involving summation of amplitudes along diffraction hyperbolas is founded on the integral form, and a finite-difference method pioneered by Jon Claerbout, Stanford University, approximates direct solution of the differential form. After discussing fundamental assumptions required for implementation of the two migration approaches, this paper focuses primarily on comparison migrations of both synthetic data and of marine and land profiles. For good data of modest spatial dip, the two approaches produce similar results. However, the seismic trace spacing (receiver group interval) is found to play different, but fundamental, roles in governing the accuracy and signal-to-noise quality of both types of migration.

$$
2: 25
$$

NN2. Effect of wavelet processing on stratigraphic resolution. R. G. Rives and J. R. Fratch (GeoQuest International, Ltd., Suite 130, 4605 Post Oak Pl., Houston, TX 77027)

Wavelet processing has proved to be a viable technique for improving the resolution and character of seismic events. Substantial improvement in signal-to-noise ratio, better continuity and stability of events are obtained with this technique. As a result of this improvement a more meaningful stratigraphic interpretation is available to the geophysicist. Evi- 
dence of this improvement in data quality has been the close agreement between well data and wavelet processed data. This agreement is possible because the effects of ghosting, instrument and system responses are removed from the seismic data. Also, accurate stacking velocities obtained with this technique normally help to improve the primary-to-multiple ratio on the stacked sections. Owing to this technique there is increased stratigraphic resolution in marine data which has resulted in more reliable quantitative methods for relating the reflection coefficients and the polarity of reflections to lithology. Current subsurface modeling techniques and other recent developments along these lines are providing insights which are principally of a stratigraphic nature.

\section{$2: 50$}

NN3. Synthetic aperture arrays in sonar and seismic profiling. Arthur B. Baggeroer (Departments of Ocean and Electrical Engineering, Massachusetts Institute of Technology, Cambridge, MA 02139)

The impressive results of applying the synthetic aperture concept in radarf has stimulated interest in employing it for mapping the seabed and the seismic structure beneath it. In sonar, several studies have proposed modifications of side scan systems to implement this concept; and in seismics, it is embodied in the operation of migration. These two applications have evolved independently; nevertheless, it appears that each could benefit from the other in solving related problems, particularly with regard to the signal processing. In this presentation we indicate areas of mutual interest in the signal processing of these two applications of the synthetic aperture concept by exploring the similarities and differences from a general array processing perspective.

NN4. Performance evaluation of a high-resolution sub-bottom profiling system. B. G. Watters and J. E. Barger (Bolt Beranek and Newman Inc., Cambridge, MA 02138)

The design and performance of a modern digital high-resolution sub-bottom profiling system are reviewed. The system is designed to identify drilling hazards in about the first $3000 \mathrm{ft}$ of sea floor. Performance comparisons with a conventional active sonar are made using metrics such as figure of merit. The sound source is a sparker; the receiver a towed line array. There are two stages of signal processing: the first stage is applied to each hydrophone channel, and the second stage in the combination of all channels. The first stage includes pulse compression (zero phase least-squares Wiener filtering using a source monitoring hydrophone system), the removal of surface-images ("deghosting"), and the reduction of bottom-surface multiple reflections ("dereverberation"). Two near-field movingfocus beam steering second stages are compared; CDP (common depth point) stacking and Tucker et al.'s within-pulse electronic sector scanning. Typical sub-bottom records will be shown and the effectiveness of various processing steps illustrated. Also discussed are techniques for quantifying reflection coefficients to aid in lithologic interpretation and of "seismic modeling" to help unravel records with overlapping returns.

$$
3: 40
$$

NN5. Role of the stationarity equation in linear least-squares estimation, spectral analysis and wave propagation. L. C. Pusey (Chevron Oíl Field Research Company, P.O. Box 446, La Habra, CA 90631) and A.B. Baggeroer (Department of Electrical Engineering, Massachusetts Institute of Technology, Cambridge, MA 02139)

We develop the properties of the innovations representation for continuous-time stationary processes and consider applications to spectral estimation and the description of wave propagation in a lossless nonuniform medium. Both applications are based on the Levinson recursion for the causal least-squares estimation filter and the continuous-time counterpart of this recursion, the stationarity equation. In the first application, we develop an unconstrained representation of positive definite extensions by observing that the stationarity equation completely characterizes the least-squares estimation filter for stationary processes. This representation is incorporated in a noval approach to spectral estimation whereby the maximum entropy method is generalized so as to include arbitrary positive definite extensions of the estimated covariance. In the second application, we observe that the stationarity equation is descriptive of wave propagation in a lossless nonuniform medium and use this fact to obtain an interesting time-domain solution of the nonuniform wave equation. The solution is an extension of discrete-time results in seismology concerning acoustical wave propagation in a layered medium.

NN6. Nonlinear acoustics of water-saturated marine sediments. Leif Bjørno (Department of Fluid Mechanics, Technical University of Denmark, Building 404, DK-2800 Lyngby, Denmark)

Interest in the acoustic qualities of water-saturated marine sediments has increased considerably during recent years. The use of sources of high-intensity sound in oil propsecting, in geophysical and geological studies of bottom and subbottom materials and profiles and recently in marine archaeology has emphasized the need of information about the nonlinear acoustic qualities of water-saturated marine sediments. While the acoustic experiments and theoretical investigations hitherto performed have concentrated on a determination of the 
linear acoustic qualities of water-saturated marine sediments, their parameters of nonlinear acoustics are still unexplored. The strong absorption, increasing about linearly with frequency, found in most marine sediments and the occurrence of velocity dispersion by some marine sediments restrict the number of nonlinear acoustic test methods traditionally available for fluids to a few being used for a determination of the nonlinear acoustic parameters of water-saturated marine sediments. These test methods, comprising static, thermodynamic and finite-amplitude wave distortion and absorption methods, aiming at a determination of $B / A$ for marine sediments, are given a critical evaluation with emphasis put on limitations in their applicability. $B / A$-values of some marine sediments-quartz medium and fine sand, silt, and clay - characterizing the seabed of the Danish archipelago and measured in our laboratory by means of the test methods discussed are given and are compared with nolnlinear acoustic qualities determined through small-scale explosion tests performed in the sediments. Sources leading to possible deviations between test results arising from prospective in situ measurements and from measurements carried out under laboratory conditions are discussed.

\section{Contributed Papers}

$4: 30$

NN7. Sub-bottom profiling with hydroshock impulse source. J. V. Bouyoucos, J.K. McLaughlin, Jr., and D. E. Nelson (Hydroacou stics, Inc., P.O. Box 3818, Rochester, NY 14610)

Hydroshock is a form of impulse source in which a controlled, imploding flow is converted to an acoustic impulse without after shot clutter. Hydroshock MI was used recently in an experiment with a short hydrophone line array to examine the sub-bottom returns from Seneca Lake. The source and receive array were towed near the surface over regions where the depth of the lake was approximately $500 \mathrm{ft}$. Sub-bottom structure to sub-bottom penetrations in excess of $600 \mathrm{ft}$ was delineated. The experiment will be described, and the contributions of the source waveform to the resolution of sub-bottom structure will be discussed. This research has been supported by the Office of Naval Research, Code 222.

\section{$4: 42$}

NN8. Geometrical and environmental constraints singlechannel seismic record interpretation. (Sea Floor Division, Naval Oceanographic Laboratory, Naval Ocean Research and Development Activity, Bay St. Louis, MS 39520)

Despite the fact that much better seismic data are presently being collected in the deep oceans than was thought possible only two years ago, the present bulk of qualitative and quantitative information about the structures and sediments of the ocean basins resides in the estimated 10 million track-kilometers of archived single channel seismic data. An enormous amount of invaluable information can be extracted from this resource if the geophysicist/geologist considers the limitations placed on the interpretation of the data by the conditions under which they were collected. The identification of multiples, diffractions, bubble pulses, and velocity effects must be accomplished before the effects of vertical exaggeration can be removed, true dips calculated, features migrated to their true position, and reflector separations more closely approximated. Examples show how each of these data processing steps can be accomplished.

\section{$4: 54$}

NN9. Effective low-frequency geoacoustic properties of ocean sediments inferred from acoustic reflectivity measurements and corresponding regional geological data. R. L. Dicus, R.S. Anderson, and O.I. Diachok (Naval Ocean Research and Development Activity, Bay St. Louis, MS 39520)

Acoustic reflectivity measurements were made at five deep ocean sites and processed for the ocean bottom impulse re- sponse at low frequencies $(<200 \mathrm{~Hz})$. Some of the essential features of the acoustic reflectivity at each of the sites were modeled in terms of a geoacoustic model consisting of a single thick $(>50 \mathrm{~m})$ sediment layer with a scund velocity gradient. The parameters for the model, viz., layer thickness, sediment interfacial sound velocity, and average gradient, density, and attenuation, were evaluated by theoretical analysis of the computed impulse responses. A compilation and analysis of the existing regional geological data from sources as gravity cores, deep drill cores, sonobuoy measurements, and scismic profiler records were also made to develop predictions of the model parameters. The sensitivity of acoustic bottom loss functions to variations in sediment parameters is discussed in view of the generally small differences obtained between the geological predictions and the acoustically inferred values.

\section{$5: 06$}

NN10. Toward a quantitative near-bottom seismic profiler. Robert C. Tyce (Marine Physical Laboratory, Scripps Institution of Oceanography, San Diego, CA 92093)

This paper describes the development and application of a system for quantitative near-bottom stismic profiling: at 4 $\mathrm{kHz}$. Developed as part of the Deep-Tcw Instrumentation System of the Marine Physical Laboratory, this system represents a capability which is relatively unique in terms of lateral resolution. This capability was developed in order to observe small-scale horizontal variations in acoustic reflectivity of the sea floor as well as similar properties for shallow, layered structures in bottom sediments. The system provides realtime computer processing and quantitative displays of processed data in several forms. These displays include a highresolution grey scale display of equivalent plane wave pressure (compensating for Fish altitude), a thræe-dimensional contourogram display of equivalent intensity, and a plot of integrated equivalent energy for travel time sections (typically corresponding to $0-5$ and $5-55 \mathrm{~m}$ into the bottom). These quantitative displays provide a real-time capability for mapping reflectivity of the sea floor and subbottom reflectors within a survey area and for estimating; acoustic attenuation in marine sediments from observations of: layer reflectivity as a function of depth of burial. Such dete:cminations of attenuation can be made directly from the corrposite computer display which is produced on a facsimile recorder. The data from this system suggest that small-scale variations are common to many areas of the sea floor, with poorly explained variations of up to $10 \mathrm{~dB}$ over lateral distances of less than $50 \mathrm{~m}$. Values of effective attenuation determined by this system show good agreement with values measured directly in the San Diego Trough, and suggest unusually low values for highly calcareous sediments on the Carnegie Ridge. 


\title{
Session 00. Engineering Acoustics V: Acoustic Arrays
}

\author{
Harry B. Miller, Chairperson \\ Naval Underwater Systems Center, New London, Connecticut 06320
}

\section{Invited Papers}

$2: 00$

O01. Electronically steered parametric transducer with variable frequency. D.W. (Applied Physics Laboratory, The Johns Hopkins University, Laurel, MD 20810)

Using digital techniques, the beamsteering and signal-generation electronics for a parametric array can be packaged in a smaller volume than the array itself. This leads to a new concept for transducers in which these electronics are considered to be part of the transducer. Such a steerable parametric transducer was designed by APL and proper operation was demonstrated during in-water tests in June of 1975. The drive waveform for the transducer was generated by pulse width modulating a $21-\mathrm{kHz}$ square-wave carrier to produce two primary drive frequencies. The difference freqeuncy (nominally $5 \mathrm{kHz}$ ) generated by nonlinearities in the water could be held at a particular frequency or could be readily chirped. Electronic beamsteering in two dimensions was achieved by providing a rariable time delay for each element of a 2-dimensional 61 -element array. The variable delay was achieved by varying the rate at which the modulated carrier was clocked through a matrix of shift registers. The nonlinear beam could be steered to a particular angle or scanned by linear variation of the clock frequency. [Work supported under Navy Contract No0017-72C-4401.

\section{Contributed Papers}

$$
2: 30
$$

OO2. Band elimination processor for an experimental parametric acoustic receiving array. D. F. Rohde, T. G. Goldsberry, W.S. Olsen, and C.R. Reeves (Applied Research Laboratories, The University of Texas at Austin, P. O. Box 8029, Austin, TX 78712)

In the Parametric Acoustic Receiving Array (PARRAY), a low-frequency acoustic wave modulates a locally generated high-frequency carrier wave. The information in the low-frequency signal wave then appears as low-level, modulation side bands of the carrier. The function of the receiver electronics is to suppress the high-level carrier while simultaneously amplifying the low level near side-band signals. $A$ band elimination processor has been developed that is capable of detecting signals with carrier to side-band ratios approaching $180 \mathrm{~dB}$. Caseaded erystal filters and lowmnoise amplifiers provide the high order to carrier suppression. Additional circuitry is incorporated to permit independent observation of the upper and lower side bands. The band elimination processor is described in detail and test data for side-band signals $40 \mathrm{~Hz}-4$ $\mathrm{kHz}$ from a $65-\mathrm{kHz}$ carrier are presented. [This research was supported by the Advanced Research Projects Agency of the Department of Defense and was monitored by the Naval Electronic Systems Command under Contract NO0039-76-C-0231.]

$$
2: 45
$$

003. Crystal-controlled pump signal source for an experimental parametric acoustic receiving array. W.S. Olsen, T.G. Goldsberry, C.R. Reeves, and D.F. Rohde (Applied Research Laboratories, The University of Texas at Austin, P.O. Box 8029, Austin, TX 78712)

In the Parametric Acoustic Receiving Array (PARRAY), a locally generated, high-frequency carrier (or pump) wave is modulated in the water by a low-frequency acoustic wave. The information in the low-frequency signal appears as low-level modulation side bands of the pump wave; therefore, the sideband noise of the pump-signal source is an important PARRAY system parameter. Since commercial oscillators with adequate spectral purity were not available, a program was initiated to develop a $65-\mathrm{kHz}$ crystal-controlled oscillator with sufficient spectral purity for use as the pump-signal source in an experimental PARRAY. Application of state-of-the-art techniques and components has enabled the design and construction of a erystal-controlled oscillator with spectral purity previously unattainable in this frequency region. Spectral purity of this oscillator is approximately four orders of magnitude better than commercially available oscillators in the $65-\mathrm{kHz}$ frequency region. Measurements performed on the oscillator show that the spectrum level side-band noise referenced to the carrier level is better than $-\mathbf{1 7 0} \mathrm{dB}$ for frequencies greater than $100 \mathrm{~Hz}$ away from the carrier. The oscillator design is described in detail and side-band noise test data are presented and discussed. (This research was supported by the Advanced Research Projects Agency of the Department of Defense and was monitored by the $\mathrm{N}$ aval Electronics Systems Command under Contract NO0039-76-C-0231. ]

$$
\text { 3:00 }
$$

004. Finite-amplitude radiation fields of a plane-piston projector. F. H. Fenlon (Applied Research Laboratory, The Pennsylvania State University, State College, PA 16801) and J.W. Kesner (Westinghouse Electric Corporation, Oceanic Division, Annapolis, MD 21404)

A new form of Burgers' equation for a plane-piston projector which unites the previously established plane and spherical waveforms [D. T. Blackstock, J. Acoust. Soc. Am. 36, 217219(L) (1964)] will be consîdered. One of the most significant features of this equation is its ability to describe the amplitudedependent transformation of purely spherical shock waves to mixed plane-spherical shocks, and finally to purely plane shock waves which exhaust themselves via thermoviscous and finiteamplitude losses within the nearfield region of the projector. The effect of such transitions on the harmonic fields of an initially monotonic finite-amplitude wave will be reviewed. On the basis of recent experiments [P.J. Welton, private communication], it will also be shown that the new equation provides a much more accurate representation of difference- 
frequency radiation within the "nearfield" of low-frequency "diffraction-limited" parametric arrays than certain alternative approximations. [Work supported by ARPA. ]

\section{3:15}

O05. Analysis for fill-time effects on cylindrical arrays. R. H. Stokes (Applied Research Laboratories, The University of Texas at Austin, P.O. Box 8029, Austin, TX 78712)

When a plane wave impinges on a cylindrical array, the leading edge insonifies the first few elements of the array. As the wave front continues past the leading edge of the cylinder, more and more elements are insonified until all elements in the array are insonified. This time between the first and last element to be insonified is called array "fill time." For a phase-shift beamformer, where the insonifying pulse length is long compared to the fill time, the resultant beam pattern is essentially the same as from a planar array of similar aperture or a cylindrical array with a delay line beamformer. When the pulse length and the fill time are approximately the same, there has been no detailed description of how the beam pattern is affected. Accordingly a thorough analysis of array fill time has been made of a cylindrical array, phase-shift beamformer in comparison to a delay line beamformer and to a planar array. The conclusion reached from this study is that there is little effect on the array beam pattern due to the fill time as long as the fill time is no greater than the pulse length. [Work supported by NCSL. 1

$$
3: 30
$$

006. Analysis of passive synthetic-aperture arrays for nearfield measurement of large moving noise sources. Anthony J. Rudgers (Code 8152, Naval Research Laboratory, Washington, DC 20375)

Passive synthetic-aperture arrays can be used to measure, in the nearfield, the farfield characteristics of large moving noise-radiating sources. A planar synthetic-aperture array is created when such a source moves at uniform speed past a line receiving array. Trott-array shading is applied to the receivers comprising the line array. The time-varying signals, which are generated by the source at each receiver, are likewise processed using Trott-array shading functions. This results in a virtual Trott receiving array many times the length of the moving source. The characteristics of the virtual array are equivalent to those of a physical array of the same size, with the noise source fixed within its plane-wave region.

\section{$3: 45$}

007. Numerical investigation of an acoustic slow waveguide. Benson Jay King (Code 8151, Naval Research Laboratory. Washington, DC 20375)
The effect of a cylinder of material whose sound speed is less than that of water on the radiation beam pattern of an omnidirectional sound source is incestigated. Specifically a point source is placed near one end of the cylinder. This cylinder serves as an acoustic waveguide and enhances the radiation from the source in the direction of the waveguide. The waveguide is mathematically modeled by use of the surface Helmholtz integral equation together with the requirement of continuity of acoustic pressure and nolmal velocity on the surface common to the waveguide and surrounding water as reported by P.H. Rogers and W.J. Trot: [J. Acoust. Soc. Am. 56, 1111-1117 (1974)]. The integral equations are solved numerically. Beam patterns and directivity gains for waveguides of various lengths and diameters are calculated. Included are results for composite waveguides consisting of an inner core of one material surrounded by an outer shell of another material. (Work supported by Naval Electronic Systems Command, Code 320.1

\section{$4: 00$}

O08. Experiments with cavitating parkmetric sources. Mark B. Moffett and William L. Konrad (Naval Underwater Systems Center, New London Laboratory, New London, CT 06320)

A cavitating parametric source utilizes a field of collapsing cavitation bubbles created by an intense two-frequency primary sound field. Since the cavitation zone is modulated at the difference frequency, this frequency (and its harmonics) is generated. The beam pattem is determined by the shape of the cavitation zone while the source level depends on the size of the zone. Experimentation with a number of sources utilizing face cavitation and remote cavitation has resulted in a better understanding of the performances of these devices. Cavitation thresholds for pure-tone and dual-frequency primary exeitation, have been found to be nearly identical. [Sponsored by Naval Material Command. I

\section{$4: 15$}

009. Underwater acoustic television transmission via the parametric source. W. L. Konrad (New' London Laboratory, Naval Underwater Systems Center, New London, CT 06320)

The narrow-beam wide bandwidth characteristics of the parametric difference radiator are particularly advantageous when applied to wide-band, multipath sensitive transmissions such as graphics or television. The reatults of a televisiontransmission test conducted over a range of $4 \mathrm{~km}$ at the NUSC Seneca Lake facility are described. [Work supported by NUSC.]

\title{
Session PP. Physical Acoustics VI
}

\author{
W.G. Neubauer, Chairperson
}

Naval Research Laboratory, Washington. D.C. 20375

\section{Contributed Papers}

$$
2: 00
$$

PP1. Ultrasonic-transducer power output by modulated radiation pressure, M. Greenspan, F. R. Breckenridge, and C. E.
Tschiegg (Institute for Basic Standards, National Bureau of Standards, Washington, DC 20234]

We have set up and are using an apparatus for the measure- 
ment of total sound power output of a piezoelectric transducer radiating into water. This apparatus combines the better features of previously used methods which depend on radiation pressure. The input is modulated at a low frequency and the output power is intercepted by an absorbing target which experiences a force at the modulating frequency. The target is mounted on the armature of an electromagnetic microphone provided with an independent coil through which a current at the modulating frequency is adjusted in amplitude and phase, either manually or automatically by feedback, to arrest the motion of the armature. Under these conditions the force depends only on the current, and the apparatus can be calibrated using direct current and dead weights. It is thus absolute. In practice, the carrier frequency is swept over any part of the range $1-80 \mathrm{MHz}$ while a recording of power output versus frequency is made. Examples of curves from normal and defective transducers will be shown. Other applications, as in medical dosimetry will be discussed.

\section{$2: 15$}

PP2. Use of an impedance tube as a standard sound-power source, T. M. Proctor and R. K. Cook (Institute for Basic Standards, National Bureau of Standards, Washington, DC 20234)

In 1966 William A. Jack proposed the use of an Impedance tube as a standard sound-power source for measurements in reverberation rooms. He proposed the use of the pressure standing-wave ratio to determine power radiated from the tube's open end. We measured the time-averaged intensity (Umov) vector in the tube by multiplication of sound pressure and particle velocity. Two small electret microphones separated by a known distance and a small analog computer form the basis for this measurement. The pure-tone results for the limited range of the tube $(300-1000 \mathrm{~Hz})$ are compared with sound power measurements done in the free field. Such comparisons are made with the aid of the theoretical radiation pattern of an open tube as determined by Levine and Schwinger. Edge effects at the mouth of the tube were examined. The impedance-tube source is also compared to the results of sound-power measurements made in the reverberation chamber.

$$
2: 30
$$

PP3. Sound-intens lty patterns for vibrating plates, J. Daniel Brito and R. H. Lyon (Department of Mechanical Engineering, Massachusetts Institute of Technology, Cambridge, MA 02139) and Jerome E. Manning (Cambridge Collaborative Inc., 238 Main St. , Cambridge, MA 02142)

The spatial patterns of sound intensity from a baffled, simply supported plate are determined theoretically and experimentally. The first is obtalned by a modification of Rayleigh formula and the second by scanning with an accelerometer-microphone pair and digitally processing the signals. Contours of equal intensity are developed from computer simulation, that show zones of energy radiation and zones of energy absorption. The space-average radiation efficiency is found to agree with previous results obtained from farfield equations and with experimentally determined values obtained from reverberant field measurements, for single modes and for $1 / 1-$ and $1 / 3$-oetave band excitation.

\section{$2: 45$}

PP4. Extension of scaling laws for model experiments. M. C. Junger (Cambridge Acoustical Assoclates, Inc., 1033 Massachusetts Ave., Cambridge, MA 02138)

Of the four dimensionless ratios required to characterize acoustic data (I. C. Romer, Jr. , J. Acoust. Soc. Am. 59, 1227-1229 (1976) ] two depend exclusively on the physical properties of the acoustic fluid. A third ratio is kept invariant in the usual scaled models, wher eby dimensions $L$ and frequencies $f$ are modeled respectively, as $L / s$ and $s f$. A fourth ratio is the "sonic Reynolds Number", $\rho L f / \mu$, which controls boundary layer phenomena. It remains invariant if linear dimensions are scaled as $L / s^{1 / 2}$. Reflection and absorption by Rayleigh solids, and other boundary phenomena can be modeled by scaling boundary statistics, e.g. . boundary roughness, and interstitial spaces in porous layers (fibrous blankets and sandy ocean bottoms) as $L / s^{1 / 2}$, while scaling macroscopic dimensions, e.g. , layer thickness, as $L / \mathrm{s}$. (Work supported by USN Office of Naval Research.]

\section{$3: 00$}

PP5. Bubble resonance in a water-cooled reactor, $T$. E. Burton and W. L. Whittemore (General Atomic Co. , TO-170, P. O. Box 81608, San Diego, CA 92138)

General Atomic's TRIGA research reactor is essentially an array of cylindrical fuel rods of conventional geometry at the bottom of a large pool of water, cooled by natural convection and operated at power levels up to 2.0 MW. There can be some local boiling at higher reactor powers. Of many TRIGA reactors of similar design now in operation, only one makes an objectionable noise. Frequency spectral analysis of microphone data suggests that the source of the noise is volume oscillation of gas bubbles near the core, resonating with a standing acoustic wave in the pool. The bubble-oscillation natural frequency varies inversely with aggregate-bubble volume. Resonance has been almost eliminated by improving the coolant flow and reducing the aggregate-bubble volume. Investigations into the problem and its solution are continuing.

\section{$3: 15$}

PP6. Schlieren imaging of unipolar acoustic pulses, J.A. Bucaro, L. Flax, H. D. Dardy, and E. F. Carome (Naval Research Laboratory, Washington, DC 20375)

The schlieren observation of broadband acoustic pulses is considered. The optical intensity distributions in both the image and the Fourier transform plane (stop plane) are discussed. Experimental observations are presented for Gaussianlike pressure transients generated by narrow voltage pulse $(\sim 0.1 \mu \mathrm{sec})$ excitation of thick piezoelectric plates. The intensity profiles obtained from these images are compared to calculations we have formulated for Gaussian pressure pulses. These calculations apply in the phase-grating limit and include optical diffraction effects due to a finite optical beam.

$$
3: 30
$$

PP7. Experimental studies of the propagation of wide-band acoustic impulses. E. F. Carome and M. R, Layton (John Carroll University, Cleveland, OH 44118) and J. A. Bucaro (Naval Research Laboratory, Washington, DC 20375)

Schlieren and acoustic probe techniques are being used to study quantitatively the propagation of wide-band acoustic impulses in water. Gaussian-shaped pressure impulses ( $\sim 0.1 \mu \mathrm{sec}$ wide) are generated using thick ceramic transducers. Acoustic probes capable of accurately representing the time profiles of the pressure impulse are being used in conjunction with fast Fourier-transform techniques to obtain information that may be correlated with the results of high-resolution schlieren measurements. These two methods complement one another and their simultaneous use yields interesting new information on acoustic diffraction, scattering, and other propagation phenomena. [Supported in part by the Office of Naval Research. ]

$$
3: 45
$$

PP8. Acoustic nearfield of a baffled piston using Fourier integral techniques. P. R. Stepanishen (Department of Ocean Engineering, University of Rhode Island, Kingston, RI 02881)

A method is presented to evaluate the acoustic nearfield of a baffled piston of arbitrary shape. The method is based on first transforming the well-known Kirchhoff surface integral representation of the field into a Fourier integral. The Fourier integral is then either numerically evaluated via the use of FFT algorithms or analytically evaluated at large frequencies via the use of applicable asymptotlc techniques. The relative 
advantages and disadvantages of both techniques are diseussed. It is noted that the FFT technique is more useful in the lowto mid-normalized frequency range; however, the asymptotic technique is most useful in the high frequency range. In order to illustrate the method, the nearfield of a circular and square piston is investigated using the method. The characteristics of the fields are readily obtained and shown to be in agreement with earlier results. ['This work was supported by a grant from NIH. I

\section{$4: 00$}

pp9. Reflection instabilities of large-amplitude acoustic waves, J. A. Clark (Acousto-Optics Laboratory, Catholic Univer sity of America, Washington. DC 20064)

Effects of the phase reversal which occurs when largeamplitude acoustic waves are reflected by a pressure-release boundary have been investigated experimentally. Large-amplitude, waterborne acoustic waves were generated by an induction type of electroacoustic transducer. Instantaneous pressure distributions associated with the propagating acoustic waves were determined by a whole field optical measurement method described elsewhere [Whole Field Measurements of AcoustoOptic Refraction, J. A. Clark, J. Sound Vib. 46, 306-310 (1976) I. Nonlinear deformations of the acoustic waves were analyzed both before and after reflection at the pressurerelease boundary in order to verify theoretical predictions that large-amplitude sawtooth waves will deform back into sinusoidal waves under these conditions. [Research supported by ONR. 1

\section{$4: 15$}

PP10. Propagation of acoustic waves in steady extensional flows on non-Newtonian fluids. W. H. Schwarz and R. L. Powell (Department of Mechanies and Materials Science, The Johns Hopkins University, Baltimore, MD 21218)

An analysis for the calculation of the attenuation and sound speed of a plane acoustic wave propagating through a nonNewtonian fluid that is undergoing a simple steady extensional motion is presented. The constitutive representation for the stress tensor in terms of the deformation gradient is developed for the model of a simple fluid. An explicit form is obtained for the superposed acoustic motion on the basic flow by using a functional Taylor's series having linear forms that are represented by integrals whose kernels are relaxation functions that depend on the extensional rates. An alternative representation is given as rate-dependent complex viscosities that depend on the relative orientation of the acoustic vector to the axes of extension. Several examples are described that are experimentally realizable and that can provide information on the rheological nature of the non-Newtonian fluid. [Work supported by NSF. 1

$$
4: 30
$$

PP11. Vector sound. Richard $W$. White (Naval Undersea Center, San Diego, CA 92132)
Vorticity in linearly viscous flow oteys a diffusionlike equation containing the infinite propagatior speed of its parabolic form. Under incompressible conditions, where sound speed is effectively infinite, there is probably no contradiction. For compressible flow, with finite sound speed, it is suggested that the vorticity equation be modified so as to also reflect a finite propagation speed. An attempt to accomplish this leads to an assult on one assumption entrenched in the lore of acoustics, that of strictly longitudinal sonic waves. The consequences are examined and implications for aeroacoustics and turbulence theories are considered.

\section{$4: 45$}

PP12. The acoustic monopole in motion. T. D. Norum and C. H. Liu (NASA-Langley Research Center, Hampton, VA 23665)

A widely used experimental version of the acoustic monopole consists of an acoustic drive of restrioted opening forced by a discrete frequency oscillator. To investigate the effects of forward motion on this source, it was mounted above an automobile and driven over an asphalt surlace at constant speed past a microphone. The received signal was compared to results computed from an analysis of a fluctuating-mass-type point source moving above a finite imfedance reflecting plane. Good agreement was found between experiment and theory when a complex normal impedance representative of a fairly hard acoustic surface was used in the analysis. Nonuniform motion of the source was also considered by analyzing the monopole moving with constant acceleration in free space. Computation of the observed signal indicates that deviations from the constant velocity case become noticeable only at rather large values of acceleration.

$$
\text { 5:00 }
$$

PP13. Vortex simulation of the pressure field for a jet. Y.T. Fung and C. H. Liu (NASA-Langley Research Center, Hampton. VA 23665)

Variations of the pressure field of a jet are simulated numerically by axisymmetric vortex ring's with viscous cores submerged in an inviscid uniform streim. The results are compared with the exper imental data measured near a jet. The intervals of shedding time between successive vortex rings are first matched with time intervals between successive pressure peaks of the experiments. and then generated by a numerical program to simulate the randomness of the pressure distribution in the acoustic field. This numerical program randomly generates shedding time intervals with the same probability distribution as that given by those measured in the experiments. Statistical comparisons between numerical and experimental results including probability distributions of shedding periods between successive peaks and variations of peak time period of vortex shedding along the cone of measurement are made up to slx diametersi downstream of the jet. The role played by the axisymmetric model in the pressure field is also discussed. 


\title{
Session QQ. Psychological and Physiological Acoustics VII: Precis Poster Session on Hearing: Threshold, Binaural, Impairments, Reflexes and Measurement
}

\author{
P.A. Neff, Chairperson \\ Navy Regional Medical Center, San Diego, California 92134
}

\section{Poster Session}

$8: 30$

QQ1. Diffuse field sensitivity of external ear based on reciprocity principle. E.A.G. Shaw (Division of Physics, National Research Council of Canada. Ottawa, Ontario, Canada K1A OR6)

The reciprocity principle defines a general relationship between transmission and reception in an acoustical system. By applying this principle, the transformation of sound pressure level from a diffuse sound field to the eardrum can be expressed in terms of the frequency, the radiation impedance $Z_{\text {rad }}$ of the external ear looking outward from the eardrum position, and the load impedance $Z_{d}$ presented by the eardrum. The formula, which contains no assignable constants, has been used to calculate the diffuse field response curve for a physical model of the external ear combined with an eardrum impedance simulator. An impedance tube was used to measure $Z_{\text {rad }}$ and $Z_{d}$. The magnitude of the response and the frequencies of the resonance peaks are in excellent agreement with other measurements. The diffuse field pressure gain at the primary resonance frequency $(2.7 \mathrm{kHz})$ is approximately $18 \mathrm{~dB}$.

$$
\text { 8:34 }
$$

QQ2. Diffraction of impulse noise by head shadow. R. J. Keim (Department of Otorhinolaryngology, University of Oklahoma, P.O. Box 26901, Oklahoma City, OK 731 90)

The frequency dependent aspects of sound shadow effect on steady state noise have been well established. Little is known of this relationship for impulse noise because of inaccessibility of controlled subjects. Pre and post exposure pure tone thresholds were studied in 23 selected subjects who completed an M-16 rifle qualification course. All had identical noise exposure conditions. Significant differences between $r$ ight and left ear thresholds were demonstrated and agreed with previous reports on sound shadow. [K. D. Kryter and G. Garinther, Acta Oto-Laryngol. Suppl. 211 (1965); R. J. Keim, Arch Otolaryngol. 90, 65-68 (1969) . I Attenuation character istics of the subjects exposed to impulse noise were compared with those determined in the laboratory IF.M. Weiner and D. A. Ross, J. Acoust. Soc. Am. 18, 401-408 (1946)] for steadystate noise. Statistically significant correlation was determined only when external canal resonance features were also considered in the calculations of attenuation. The results indicate that sound shadow affects impulse noise in the same way as observed for steady state noise and that the "acoustic notch" is primarily attributable to canal resonance features.

$$
8: 38
$$

QQ3. Eardrum pressure at absolute threshold: Where is the "missing $6 \mathrm{~dB}^{\text {"? }}$ Mead C. Killion (Industrial Research Products, Inc., a Knowles Company, 321 N. Bond, Elk Grove Village, IL 60007)

Eardrum pressures at hearing threshold have been calculated from both earphone data (ISO R389-1964 and ANSI S3.6-1969) and free-field data (ISO R226-1961). When head- and ear-diffraction effects and an apparent error in ISO R226 are ac- counted for in the free-field data, and real-ear versus coupler differences and physiological noise are accounted for in the earphone data, the agreement bctiveen the two derivations is excellent. At the audiometric frequencies of $125,250,500$, $1000,2000,4000$, and $8000 \mathrm{~Hz}$, the estimated eardrum pressures at absolute threshold are $30,19,12,9,15,13$, and 17 dB SPL, respectively, At the middle frequencies-where the comparisons should be on a solid footing-no evidence of the "missing $6 \mathrm{~dB}$ " is seen. an observation consistent with the experimental results of several recent studies.

\section{$8: 42$}

QQ4. Thresholds for apparent auditory motion induced by linearly changing interaural time differences. C. M. Brandauer (Bell Laboratories, 11900 N. Pecos, Denver, CO 80234) and Wayne Ward (Department of Psychology, University of Colorado. Boulder, CO 80302)

That listening dichotically to two waveforms differing slightly in frequency produces an iniage that appears to move from one ear to the other is well known le.g. , Lord Rayleigh (J. W. Strutt), Philos. Mag, Ser. 6 13, 214-232 (1907)]. We have determined the thresholds for apparent auditory movement using low-pass-filtered pulse trains by changing the interaural time difference $T$ by an increment $t$ with each dichotic pulse pair. Two major experimental paradigms were used with several pulse repetition rates and initial values of $T$. In the first paradigm, $t$, a measure of movement rate, was the independent variable and $N$, the number of pulse pairs or movement time required for the movement threshold was determined. The second paradigm reversed the roles of $t$ and $N$, thus yielding the required movement rate for a given movement time.

\section{$8: 46$}

QQ5. Binaural frequency response function. A A humada, Jr. (Department of Aeronautics and Astronautics, Stanford University, Stanford, CA 94305)

The relative sensitivity of the binaural lateralization process to neighboring frequencies was measured using $50-\mathrm{Hz}$ bandwidth noise bursts. The 300 -msec bursts had 16 pure tone components spaced $3.33 \mathrm{~Hz}$ apart and in random phase. Bursts had center frequencies of $100,200,300$, or $400 \mathrm{~Hz}$. Amplitudes of the components in decibels were varied linearly in log frequency with slopes that ranged from -36 to $60 \mathrm{~dB}$ octave in 12-dB steps. The noise burst presented to one ear differed from that simultaneously presented to the other ear only in that the eight higher frequency components were phase shifted corresponding to a 1 msec delay in one ear and the eight lower components were phase shifted to give a 1 -msec delay in the other ear. Observers listened to a binaural burst, $300 \mathrm{msec}$ of silence, and then the same stimulus sent to opposite ears. They judged the direction of movement to be right, left, or none. The lateralization judgments are consistent with an overall frequency response which peaks between 200 and $300 \mathrm{~Hz}$. Results are compared with neural modulation frequency re- 
sponse functions. [Work supported by Stanford-Ames Joint Institute for Aeronautics and and Acoustics. I

$$
8: 50
$$

QQ6. Anomalous lateralization in the precedence effect. H. Gaskell (Department of Experimental Psychology, South Parks Road, Oxford, OX1 3UD, England)

When two 20- $\mu$ sec click pairs are presented binaurally in rapid succession, observers hear a single sound from a location which depends mainly on the properties of the first pair (precedence or Haas effect). The effect is usually demonstrated with interaural delays but has also been obtained with interaural intensity differences. In particular, if a click pair is presented simultaneously to the two ears followed approximately $400 \mu$ sec later by a second simultaneous binauraI pair, the clicks having amplitudes $L^{1}=R^{1}=L^{2}$ and $R^{2}=0.5 \times L^{2}$, one might expect to hear the resulting single sound either centrally or on the left. However, the apparent location (measured in a standard two-alternative forced-choice lateralization experiment) in these and other nontrivial conditions is clearly to the right of the midline. It is suggested that these unexpected results oceur because the relative amplitude and phase spectra of the stimuli have frequency regions in which both amplitude and phase cues indicate that the lateralization is opposite to that suggested by the temporal waveform. Further experiments, (1) filtering out appropriate frequency bands and (2) making lowenergy regions of the spectra, support this hypothesis.

\section{$8: 54$}

QQ7. Model for the localization of sound in the azimuthal plane. George F. Kuhn (Institute for Basic Standards, National Bureau of Standards, Washington, DC 20234)

An objective study of the interaural time difference (ITD) was performed on a manikin comprised of a head and a torso. Data were taken for both a bare and a closed torso. The measured ITD's correspond reasonably accurately at the low and the high frequencies to the computed theoretical values for a rigid sphere of an effective radius $a$. The theoretical ratio of the low-frequency $(<500 \mathrm{~Hz})$ ITD to the high frequency $(>2000 \mathrm{~Hz})$ ITD is $\frac{3}{2}$. The measured ITD is a minimum between 1.4 and $1.6 \mathrm{kHz}$ for angles of incidence, $\theta_{1 \mathrm{nc}}$, of sound between $15^{\circ}$ and $60^{\circ}$. At both the low and the high frequencies the data can be expressed by universal curves when the ITD is normalized by $\left(a / c_{0}\right) \sin \theta_{\text {tac }}$, where $c_{0}$ is the speed of sound in air and $\theta_{9 n c}$ is the angle of incidence. Both the ITD and the interaural sound pressure level differ ence (ILD) show differences between measurements made with the bare torso and those with a clothed torso. These objective results support the subjective measurements of past experiments which showed that in a man there was no localization improvement below approximately $500 \mathrm{~Hz}$, poor localization between $1000 \mathrm{~Hz}$ and $2000 \mathrm{~Hz}$, and a change in the localization cue around $1400 \mathrm{~Hz}$ from ITD to ILD.

\section{$8: 58$}

QQ8. Effect of perturbations in loudspeaker directivity patterns on localization. D. Queen (Daniel Queen Associates, 5524 W. Gladys Ave., Chicago, IL 60644)

Because localization cues probably occur during the first millisecond after the radiation of a sound toward a listener, the presence of reflections within this period, having higher intensity than the direct sound, can confuse localization. While such a phenomenon is rare in nature, it may be common in multichannel sound reproduction systems where transducers have frequency-variant nonuniformities in their directivity patterns. In this paper, numerical ratings of localization error, based on directivity equations of the forms $p(\theta)$ $=2 J_{1} F(\theta) / F(\theta)^{2}$ for a single source and $\left.p(\theta)=\sin [n \pi m f \sin \theta / c)\right] /$ [ $n \sin (\pi m f \sin \theta / c)$ ] for an array; and on empirical relationships of arrival time and intensity, are computed for various combinations of listening positions, room configurations, and transducer configurations. Data suggest that small nonuniformities can produce significant localization error.

\section{$9: 02$}

QQ9. Does the hearing among people with hearing loss have a normal distr ibution? G. J. Thiessen ('Division of Physies, National Research Council of Canada, Ottawa, Ontario, K1A OS1)

We make the assumptions that a person's hearing cannot be improved by aging, noise exposure, or any other otologically pathogenic agent, and that persons differ in their susceptibility to this agent. This has the logical consequence that an initially. normal distribution of hearing in a given population cannot remain so after the action of the pathogenic agent. The distribution must become skewed. Data in the literature confirm this change in distribution. This result has the further consequence that skewness in the distribution provides no evidence as to which group is the most susceptible.

\section{$9: 06$}

QQ10. Temporal masking for listeners with high-frequency sensor ineural hearing loss. Edward A. Cudahy (National Institute of Neurological and Communicative Disorders and Stroke, NIH, Bethesda, MD 20014)

One off-frequency and two on-frequency temporal masking conditions were employed to examine temporal processing as a function of frequency in hearing-impaired and normal listeners. Forward, backward, and gap masking were measured with a $2 A F C$ procedure for a 5 -msec tonal signal in the presence of a narrowband noise masker. In agreement with previously reported data employing signals at a frequency above the masker, normals and hearing-impaired listeners displayed similar masking patterns in forward and 200-msec gap masking conditions for either a 1500- $\mathrm{Hz}$ signal with an 1175-1725$\mathrm{Hz}$ noise masker or a $2500-\mathrm{Hz}$ signal with a $2400-2800$ noise masker. Moreover, in the backward and 50-msec gap masking conditions the masking patterns for the hearing-impaired listeners were less steep than normal. In contrast to previous data for the 20-msec gap conditions, both normal and hearingimpaired subjects showed flat masking patterns. Interestingly, when the signal was below the masker in frequency $(1500-\mathrm{Hz}$ tonal signal and $2400-2800-\mathrm{Hz}$ noise nasker), the masking patterns for normal and hearing-impaired listeners were similar for all conditions. The implications of these results. for speech processing in noise by listeners with high-frequency sensorineural hearing loss will be discussed.

$$
\text { 9: } 10
$$

QQ11. Two-tone suppression in hear ing-impaired listeners. M. B. Kramer and F. L. Wightman (Auditory Researeh Laboratory, Northwestern University, Evaniston, IL 60201)

Forward-masked thresholds were measured in three listeners with precipitous high frequency losses attributed to cochlear damage. The signal was a sinusoid; the masker consisted of two sinusoidal components: one fixed at the frequency of the signal, and one at a variable frequency. It has recently been reported that with the variable masker at certain frequencies and intensities, signal threshold is lower than with this additional masker component absent [R. V. Shannon, J. Acoust. Soc. Am. 59, 1460-1470 (1976) l. This decrease in signal threshold is termed unmasking and may be interpreted as resulting from suppression of the fixed masker by the variable masker, thus reducing its masking effectiveness on the signal. This unmasking effect was not observed in hearingimpaired listeners if the variable masker was in the frequency region of the hearing loss. In normal trequency regions, unmasking effects were similar to those ubserved in normal listeners. This suggests that suppression occurs at a level of the auditory system higher than the bastlar membrane, possibly at the hair cell level. [Work supported in part by NIH Grant NS12045.]

$$
9: 14
$$

QQ12. Relations between suppression and masking in normal hearing-impaired listeners. T.M. McGee, F.L. Wightman, 
and M. B. Kramer (Auditory Research Laboratory, Northwestern University, Evanston, IL 60201)

Tone-on-tone masking patterns from simultaneous and forward masking paradigms are dramatically different. It has been suggested that some of this difference is an effect of suppression. presumably present only in the simultaneousmasking data. To study this suggestion psychophysical tuning curves were measured using simultaneous and forward-masking techniques and measures of suppression were obtained from the same subjects using Houtgast's two-component forward-masking paradigm. Both normal and hearing-impaired listeners were tested. With normal listeners, simultaneous and forward-masked tuning curves were different in the frequency regions where suppression was most evident. Simllar results were obtained from hearing-impaired listeners in regions of normal sensitivity. However, in regions of decreased sensitivity, simultaneous and forward-masking tuning curves were the same and no suppression was evident. These results suggest that much of the difference between simultaneous and forward-masking data is a result of suppression. [Work supported in part by NIH Grant NS12045.]

$$
9: 18
$$

QQ13. Psychophysical tuning curves in normal and pathological ears, Arlene Earley Carney and David A. Nelson (Hearing Research Laboratory, University of Minnesota, Minneapolis, MN 55414)

Psychophysical tuning curves were obtained for normal listeners and listeners with sensorineural hearing loss in a simultaneous tone-on-tone masking procedure. As reported by Leshowitz et al. [J. Acoust. Soc. Am. 59, S2(A) (1976)], the tuning curves for several pathological ears showed no finely-tuned portion in the frequency regions where threshold was elevated. Although two subjects demonstrated broad-band, relatively flat sensorineural losses, the pattern of their tuning curves varied markedly with signal frequency. To examine this frequency effect, and to determine the effect of overall sound pressure level on frequency selectivity, tuning curves for normal listeners were run at two levels: (1) with probe at $10 \mathrm{~dB}$ SL; (2) with probe at sound pressure levels comparable to those experienced by pathological ears. The implications of these results for frequency selectivity in hearing impaired listeners will be discussed. [Work supported in part by NIH. ]

$$
9: 22
$$

QQ14. Temporary threshold shift and exposure noise bandwidth. K. Malott (Department of Psychology, Western Michigan University, Kalamazoo, MI 49008) and W.C. Stebbins and D. B. Moody (Kresge Hearing Research Institute, and Department of Psychology, University of Michigan, Ann Arbor, MI 48104)

Audiograms were obtained from three Old World monkeys by positive-reinforcement operant-conditioning procedures. Three series of nolse exposures were administered in which the width of the exposure band was varied between 0.15 and 4 octaves, with overall level held constant within each series at 100-108 dB/SPL. In two series, the upper cutoff of the exposure band was held constant at 2 and $4 \mathrm{kHz}$ respectively. In the third series, the center frequency of the exposure band was held constant at $2 \mathrm{kHz}$. When exposure noise bandwidth was 0.15 octave, the maximum of the threshold shift versus frequency function was at or above the upper cutoff of the exposure band. When the bandwith was inoreased with the upper cutoff held constant, the maximum of the function frequently shifted toward lower frequencies and the proportion of the area under the curve below the upper cutoff increased. When the bandwidth was increased with the center frequency held constant, no consistent shifts in either direction were observed in two subjects; for the other subject the maximum shifted toward higher frequencies. The implications of these findings and their relation to the human literature will be discussed. ISupported by Research Grant NS05077 and Program Project Grant NS 05785 from NINCDS. ]
$9: 26$

QQ15. Auditory effects of 24-h exposures to noise. J. H. Mills, R. M. Gilbert, and Warren Y. Adkins (Department of Otolaryngology, Medical University of South Carolina, Charleston, SC 29401)

Groups of human observers were placed in a diffuse sound field for 26- $h$ and exposed to an octave-band noise centered at $4.0 \mathrm{kHz}$. Levels of the noise were $75,80,83$, and $88 \mathrm{~dB}$ SPL. Threshold shifts produced by the noise increased for the ftrst 4-8 h of exposure and then reached a plateau or asymptote. Threshold shifts at asymptote for test tones of 4.0 or $6.0 \mathrm{kHz}$ increased about $1.7-2.0 \mathrm{~dB}$ for every $1-\mathrm{dB}$ increase in the level of the noise above about $72 \mathrm{~dB}$. However, at the frequency of maximum threshold shift $(4.5-5.5 \mathrm{kHz}$ depending upon the observer), the increase in threshold shift was about $3.0 \mathrm{~dB}$ for every 1-dB increase in the level of the noise above $73 \mathrm{~dB}$. Complete recovery of auditory thresholds occured for every observer and required between 16 and $48 \mathrm{~h}$. Such long recovery times were observed even when the magnitude of the threshold shift at asymptote was less than $10 \mathrm{~dB}$. [Supported by NIEHS. 1

QQ16. Aural nonlinearity and auditory fatigue, James $P$. Cobb and John Erdreich (Department of Otorhinolaryngology, University of Oklahoma Health Sciences Center, Oklahoma City, OK 73190)

Differences in individual susceptibility to auditory fatigue have been ascribed to factors relating to cochlear biochemistry, changes in neural excitability, hair cell damage and overdriving of the transducer organ as evidenced by nonlinear distortion. In an investigation of the relation between fatigue and auditory nonlinearity we examined two aspects of temporary threshold shift (TTS) : (1) growth of TTS with level of the fatiguing stimulus and (2) the absolute level of T'TS for a single fatiguing stimulus amplitude. Nonlinear distortion levels for the listeners were estimated using the tone-on-tone masking paradigm. If individual susceptibility to TTS is determined by factors related to maintenance of the physiological environment in the cochlea, then these factors might mediate absolute levels of TTS in response to a fixed intensity stimulus. In this instance the rate of change of TTS with fatiguing level could be a function of the increased overloading between fatiguing levels. Conversely, if TTS susceptibility is a function the degree of overloading, then absolute levels of TTS should be proportional to nonlinear distortion. The results of this study support a relation between absolute TTS levels and nonlinear distortion.

\section{$9: 34$}

QQ17. Effects of an experimental acoustic neurinoma on stapedius reflex activity. C.A. Mangham and J. M. Miller (Department of Otolaryngology, Seattle, WA 98195)

An experimental model for controlled studies of an acoustic neurinoma will be described. The model employs nonhuman primates. It permita chronic experimentation. It is reversible. Essentially, the preparation is based upon the implantation of an inflatable Silastic rubber balloon in the VII and VIII cranial nerves in the internal auditory meatus of the monkey. Prior to and following such preparation, a systematic study of the contralaterally evoked stapedius reflex was performed. Afferent and efferent limbs of the reflex arc were studied. The effects of balloon inflation and deflation were examined. Variables studied included threshold across frequency, dynamic range, habituation, rate of rise, and recovery time. Normative data indicate the characteristics of the monkey stapedius reflex are comparable to man. Implantation reversibly affected all varlables except habituation which was permanently affected. Changes in all variables were reversibly influenced by balloon inflation and deflation. The time course and quantitative changes observed will be described. Clinical implica- 
tions and possible basic mechanisms underlying the effects of the acoustic neurinoma on auditory lunction will be discussed. [Work supported by NSO8181 and RR00166.I

\section{$9: 38$}

QQ18. Abstract withdrawn.

\section{$9: 42$}

QQ19. Abstract withdrawn.

\section{$9: 46$}

QQ20. Auditory and vestibular function in human subjects exposed to oxygen at two atmospheres absolute. P. A. Neff (NRMC San Diego, CA 92134), P. L. Hendricks (VA Hospital, San Diego, CA 92161), W. L. Hunter (Duke University, Durham, NC 27706), M. J. Rensink (NRMC San Diego, CA 92134), and W. T. Morioka and R.W. Cantrell (NRMC San Diego, CA 92134)

Experiments were conducted to test the hypothesis that intermittent exposure to oxygen at two atmospheres absolute (2 ATA) could delay the onset of pulmonary toxicity. This paper reports effects of $2 \mathrm{ATA} \mathrm{O}_{2}$ (total exposure time of $14.1-19.3 \mathrm{~h}$ ) upon the auditory and vestibular systems of five diver volunteers and effects of a nor moxic $\mathrm{N}_{2} \mathrm{O}_{2}$ mix on a sixth. Immediately after exposure four subjects had increased acuity by air conduction in the mid-frequency range. Bone conduction sensitivity increased for all six subjects. Middle-ear reflexes were depressed or absent with only partial recovery one month after the experiment for the $\mathrm{O}_{2}$-breathing subjects, but were more sensitive after exposure for the subject breathing $\mathrm{N}_{2} \mathrm{O}_{2}$. Middle-ear pressures grew markedly more variable for $\mathrm{O}_{2}$ subjects up to $72 \mathrm{~h}$ postexposure, implying decreased eustachian tube function from oxygen. Vestibular systems of four $\mathrm{O}_{2}$-exposed subjects and the $\mathrm{N}_{2} \mathrm{O}_{2}$ subject were markedly suppressed to caloric stimulation after exposure, implying pressure as the contributory çause. Ciperational application may be found in possibly sensitizing certain frequencies to improve the listener's ability to detect signals in noise. [Work supported by CIC. NRMC , and COMISUBDEVGRU ONE. ]

$$
9: 50
$$

QQ21. Ear protection on ships. A. Schwartz (Dr. A. Schwartz \& Co., Acoustical Consultants. P.O. B. 7037, Haifa, Israel), P. Munch (Ministry of Transport), and L. Naim (Ministry of Labour)

With the intention of incorporate it in a revised version of the "Regulations for the Prevention of Harmful Noise on Ships (1972)" a special "Notice to Shipowners and Shipbuilders" (SHN 4/76) was prepared by a technicial committee and issued by the Ministry of Transport, Dept. $\alpha^{*}$ Shipping and Ports. Ear protection devices are required in machinery spaces where the noise level exceeds NR 85 . The minimum required noise attenuation characteristics of the ear protection devices are: $19 \mathrm{~dB}$ at $250 \mathrm{~Hz}, 30 \mathrm{~dB}$ at $500 \mathrm{~Hz}, 36 \mathrm{~dB}$ at $1000 \mathrm{~Hz}$ and $38 \mathrm{~dB}$ at $2000 \mathrm{~Hz}$ and $4000 \mathrm{~Hz}$. Special high quality ear protection devices are required where personnel are exposed to noise levels exceeding NR 110. IThe above recommendations were published to show the way to a hisher level of crew safety and health, as envisaged in Recommendation No. 141 adopted at the 55th (maritime) session of ILO and IMCO Assembly Resolution A. 343 (IX) adopted in 1975. I

$$
9: 54
$$

QQ22. Comparative coupler and ear simulator measurements of earphones. Herman R. Silbiger (Bell Telephone Laboratories, Holmdel, NJ 07733)

A series of measurements were periormed to compare the performance of handsets and supra-aural and ear-insert-type headsets on standard and modified couplers, and on the KEMAR ear simulator. These measurements have yielded data enabling comparisons to be made of the frequency response of earphones referred to the same location in the ear, at the eardrum. The effects of ear canal resonances on the upper frequencies, as well as the effect of the imperfect seal that the earphone makes with the ear on the receiver's low-frequency response, are thus evidenced. One of the measured effects of the imperfect seal is a reduced low frequency response. The other measure of effect is leakage of room noise into the ear covered by the headphone. These effects are related to a lowering of speech transmission quality ratings as the room noise is increased, where headset types are differ entially affected. Finally, data will be presented on the equivalent free-field SPL's to SPL's measured at the eardrum for the various headset types.

\section{$9: 58$}

QQ23. Effect of earmold modifications measured by coupler, threshold and probe techniques. G. A. studebaker and R. M. Cox (Speech and Hearing Sciences, Graduate Center, City University of New York, NY 10036) and D. M. Wark (Department of Audiology and Speech Pathology, Memphis State University, Memphis, TN 38105)

The validity of probe-tube microphone measurements in providing data indicative of the magnitude of the change in the psychoacoustic experiences of subjects resulting from changes in earmold configuration was investigated. The relationship between these measures in the real ear canal and those changes observed in 2-cc and Zwislocki couplers under similar circumstances of earmold modification was also measured. Standard. vented and "open" earmold conditions were utilized. Threshold 
and probe-tube measurements were made sequentially without moving the earmold at 21-29 discrete frequencies. Statistical evaluation revealed that these two techniques did not produce significantly different results except at $125-165 \mathrm{~Hz}$ where noise masking may have been a factor influencing the threshold data. Neither coupler as used gave an accurate quantitative estimate of the effects of vented earmolds or the open ear mold configuration but the Zwislocki coupler gave a somewhat better approximation than the 2-ce coupler. [Work supported by NIH. I

\section{$10: 02$}

QQ24. Improvement and evaluation of the vibration pick-up type ear microphone. Hiroshi Ono and Shigeji Saito (Department of Otorhinolaryngology, School of Medicine, KEIO University, 35 Shinanomachi, Shinjuku-ku, Tokyo 160 Japan)

We developed a vibration pick-up-type ear microphone which was formerly reported at the.90th ASA Meeting. After evaluating its function and comparing it with other conventional microphones, we concluded as follows; It is possible to obtain remarkable sensibility without closing the ear canal and to obtain high intelligibility voice in level over $95 \%$ of sound articulation. There was no significant difference between voice intelligibility with a mask and that without a mask. Sound articulation in high ambient noise of the vibration pickup type ear microphone $(110 \mathrm{~dB}-96 \%, 125 \mathrm{~dB}-84 \%)$ is much higher than that of noise cancelling microphone $(110 \mathrm{~dB}-87 \%$, $125 \mathrm{~dB}-77 \%$ and sound pressure type ear microphoen with ear muff $(110 \mathrm{~dB}-85 \%, 125 \mathrm{~dB}-68 \%)$. By use of the earphone-microphone, we can simultaneously talk and hear through the ear, thanks to no necessity of obstruction of the ear canal. Applications of the vibration pick-up type ear microphone are (1) howlingless microphone, (2) free hand microphone, (3) mask microphone, and (4) microphone for voice communication in high ambient noise. We have made communication systems with the vibration pick-up-type ear microphone and evaluated in various environments.

FRIDAY, 19 NOVEMBER 1976

GOLDEN WEST ROOM, 9:00 A.M.

\title{
Session RR: Speech Communication VII: Analysis and Transmission
}

\author{
Edward P. Neuberg, Chairperson
}

National Security Agency, Fort Meade, Maryland 20755

\section{Contributed Papers}

9:00

RR1. Certain "perceptually consistent" spectral distance measures and their properties when applied to single formant spectra. R. Visvanathan, J. Makhoul, and W. Russell (Bolt Beranek and Newman Ine., Cambridge, MA 02138)

Recently, we proposed a class of spectral distance measures to compute the amount of deviation between two smoothed short-time speech spectra IR. Viswanathan et al. " "Towards Perceptually Consistent Measures of Spectral Distance," Proc. IEEE International Conference on Acoust. Speech Signal Process, Philadelphia, pp. 485-488, April 1976]. These measures produce results consistent with published subjective perceptual results.on formant frequency difference limen (DL). More recently, we have adjusted these measures, by a process we call "scaling," such that spectral distances due to formant frequency shifts equal to the DL at each frequency are approximately the same for different formant frequency values. In this paper, we discuss these "scaled" distance measures, and present the results of using the measures with single formant spectra. The results are in the form of plots of spectral distance obtained under different conditions of either formant frequency shift or formant bandwidth shift. Comparisons are made of these data with published subjective DL data. [Work supported by ARPA-IPTO.]

\section{$9: 10$}

RR2. Automatic detection of nasal/non-nasal feature in continuous speech, K. P. Li (TRW Defense and Space System Group, Inc., Redondo Beach, CA 90278)

Nasals usually have wider-formant bandwidth than vowels and other nonvowel sonorants. In an LPC analysis, if the last reflection coefficient is set near to unity, then all poles of the inverse filter in the $z$ plane are also shifted near to the unit circle; however, those poles do not follow the radial direc- tion. Therefore, formant frequencies found by shifted spectrum show differences from corresponding formants derived by original unshifted poles. The larger the bandwidth of the pole is, the larger is the difference between the original formant frequency and the shifted formant frequency. Nasals show particular large differences of the first and second formants. An experiment on continuous speech to classify nonvowel sonorants to nasal or non-nasal has been tested. Results are discussed.

$9: 20$

RR3. Evaluation of a voiced-unvoiced-silence analysis method for telephone-quality speech. C. E. Schmidt, L. R. Rabiner, and B.S. Atal (Bell Laboratories, Murray Hill, NJ 07974)

Recently a statistical decision approach to the problem of voiced-unvoiced-silence detection of speech was proposed by Atal and Rabiner lIEEE Trans, on Acoust. Speech Signal Process. . June (1976) l. This method was found to perform well on high-quality speech. However, the five speech parameters used in the analysis were not found to be as good for telephone-quality speech. Thus an investigation was undertaken to determine the five best parameters to use for a voiced-una reliable voiced-unvoiced-silence decision across a variety of standard telephone connections. A large number of parameters (69) were included in the investigation, including $12 \mathrm{LPC}$ coefficients, 12 correlation coefficients, 12 PARCOR coefficients, 12 LPC partial-error terms, etc. Many of the parameters were immediately eliminated because they provided almost no separability between the three decision classes. The remaining parameters were used in a knock out optimization to determine the five best parametes to use for a voiced-unvoiced-silence analysis. Varlous error welghts were investigated to see what types of errors occurred and how they could be minimized. Finally, the use of the Itakura two-pole nor- 
malization was investigated to see its affect on the error scores.

$$
9: 30
$$

RR4. Semiautomatic segmentation of speech for obtaining synthesis data. Joseph P. Olive (Bell Telephone Laboratories, Murray Hill, NJ 07974)

Recent work by Olive and Spickenagel [J. P. Olive and N. Spickengal, "Speech Resynthesis from Phoneme Related Parameters," J. Acoust. Soc. Am. 59, 99:3-996 (1976)] has demonstrated that LPC derived area functions of continuous speech can be represented by straight lines connecting the segment boundaries of the speech. Speech segments were defined as the steady state and transition portions of the phonemes in the continuous speech. The boundaries of the segments in the original experiment were determined by a trial and error procedure, and the criterion for establishing the boundaries was listening to speech synthesized from the original and smoothed parameters. In this paper we will describe a new semiautomatic approach to determine the boundaries of the segments. The method consists of fitting the original area functions by a least-square algorithm; the original boundary points used by the algorithm need be specified only approximately. Speech synthesized from area parameters obtained from the semiautomatic smoothing algorithm sounds as good as the original speech. This method will enable a faster, more accurate segmentation of speech for our work in rule synthesis of speech from dyadic units.

\section{$9: 40$}

RR5. Perceptual evaluation of several pitch detection algorithms, C. A. McGonegal, L. R. Rabiner, and A. E. Rosenberg (Acoustics Research Department, Bell Laboratories, Murray Hill, NJ 07974)

Recently an objective performance evaluation of seven pitch detection algorithms was made by Rabiner et al. [IEEE Trans. Acoust. Speech Signal Process., Oct. (1976) l. Each of the seven algorithms was compared on the basis of its performance with respect to five different types of errors. The standard of comparison was a semiautomatically determined pitch contour of each utterance. To complement this investigation we describe the results of a subjectlve evaluation of the synthetic speech quality of LPC analyzed and synthesized speech. The pitch contour used in the synthesis was either one of the seven pitch contours or the result of the semiautomatic analysis. Using a computer controlled sort board, an experiment was run in which each of eight listeners was asked to rank the nine versions of each utterance (the natural version was included to provide a stable anchor point). Results are presented on the over-all preference for each pitch detector. In addition, subject preference as a function of the pitch range of the speaker and the transmission environment used in the recording is discussed. The present results are compared to those obtained in the earlier objective performance study.

$$
9: 50
$$

RR6. Real-time harmonic pitch detector. S. Seneff (Massachusetts Institute of Technology Lincoln Laboratory, Lexington, MA 02173)

A new pitch extraction algorithm has been developed which is designed to be robust against the degradations encountered in telephone speech. The algorithm determines the pitch from the spacing between harmonics in the first formant region, using an iterative technique whereby larger peaks are weighted more heavily. The desired spectral region is obtained with better than $7 \mathrm{~Hz}$ resolution using only a 128-point FFT, after simple digital signal processing tricks. The buzz-hiss decision relies solely on the continuity of the pitch track, because standard indicators are often unreliable in the presence of noise and distortion. The algorithm has been incorporated into a real-time linear prediction vocoder implemented in the Lincoln Digital Voice Terminal, and is being evaluated on speech that has first been processed through a digital telephone simulator. [Work supported by ARPA. I

\section{$10: 00$}

RR7. Dual input noise cancellation fo: robust speech processing. Steven F. Boll (Department of Computer Science, 3160 Merrill Engineering Building, University of Utah, Salt Lake City, UT 84112)

A digital preprocessing technique has been developed for removing additive noise from speech prior to Linear Prediction analysis. The algorithm requires two signals: a primary input consisting of speech plus noise, and a second reference input consisting only of correlated noise. (The reference signal could be obtained from a second microphone placed in the noisy environment but isolated from the speaker.) Noise present in the primary signal which is correlated with that in the reference noise from the primary input. The impulse response for the reference signal filter is adaptively omputed at the analysis frame rate to minimize the energy of the difference between the primary and filtered reference signals. In addition to improving the estimate of the smoothed spectral envelope, the method can be used to improve pitch detection where inverse filtering by Linear Prediction is employed for spectral flattening.

$$
10: 10
$$

RR8. Wide-band noise reduction of noisy speech. D. T. Magill and C.K. Un (Stanford Research Instisute, Menlo Park, CA 94025)

We present a new technique that recluces wide-band noise in noisy speech. The concept of the approach is based on residual or voice excited linear predictive coding (LPC). The technique can be used in elther a digital or an analog communication link that is degraded by quantization or receiver thermal noise, or both. The technique capitalizes on two effects. (1) Linear predictive coefficients representing an all-pole vocal tract transfer function can be calculated unaffected by noise, if the noise present in speech is additive and white. (2) The signalto-noise ratio of voiced sound in the fundamental frequency region of the voice band is much higher than that in the highfrequency region. The basic procedure for conditioning noisy speech is as follows. When a voice signal that has been distorted because of quantization or thermal noise, or both, is received at a receiver, the decoded signal is analyzed to determine a set of LPC coefficients by the autocorrelation approach. The noise affects only the diaigonal elements of the autocorrelation matrix, since it is not correlated with speech. Hence, to find the coefficients that are unaffected by noise, the noise power is subtracted from the diagonal elements of the matrix. The power of white noise is reasured from a speech pause. The residual signal or noisy speech is low-pass filtered at a cutoff frequency of $600 \mathrm{~Hz}$ and then passed through a nonlinear distortion device to broaden its bandwidth. This nonlinearly processed signal is used as the input to excite the filter which has been formed by the LP' coefficients. Initial testing of the conditioner by computer simulation has yielded promising results.

$$
10: 20
$$

RR9. Piecewise linear quantization of linear prediction coefficients. C.K. Un (Stanford Research Institute, Menlo Park, CA 94025) and S. C. Yang (Chung-Shan Institute of Science and Technology, Lung-Tan, Taiwan, Republic of Chlna)

We present a new coefficient-coding method of linear predictive coding (LPC) of speech. It employs piecewise linear quantization and requires statistical properties of the LPC reflection coefficient $\left\{k_{t}\right\}$. In coding the coefficients we first construct histograms of the distribution of each coefficient values obtained from analysis of many different speakers. Then, rather than making quantization levels uniform throughout the range of coefficient values, we divide the range of each coefficient or its transformed version into three regions and assign a different number of quantization levels to each region. 
Before the range of each coefficient is divided, the minimum value $R_{0}$ and the maximum value $R_{3}$ of each $k_{1}$ are set on the basis of experimental observations. Division of each range into three regions is made by specifying $R_{1}$ and $R_{2}$ according to the distribution of coefficient values and desired quantization accuracy in each region. Although the piecewise linear coding places its importance on the density of frequencies of the coefficient values, it does not neglect the importance of the spectral sensitivity aspect. In our informal subjective listening test it was observed that the quality of synthetic speech with the transmission rate of $2.4 \mathrm{kbits} / \mathrm{sec}$ coded by piecewise quantization was equivalent to the quality with the rate of 3 $\mathrm{kbits} / \mathrm{sec}$ coded by linear quantization. The piecewise linear quantization scheme can also be used for the arc sine and log area ratio coding methods that normally employ linear quantization. In this case one can expect approximately the same amount of saving of transmission rate as for the nontransformed reflection coefficients.

\section{$10: 30$}

RR10. Best recursive fit: Some experiments on synthetic data and on real speech. Edward P. Neuburg (R5, National Security Agency, Fort Meade, MD 20755)

A synthetic signal was formed by passing a burst of energy through an all-pole digital filter, and Gaussian noise was added to the output. (This is the model of speech production that best recursive fit (BRF) was designed to analyze.) Both linear predictive coding (LPC) and BRF were used to try to recover the filter parameters; at all significant levels of noise $\mathrm{BRF}$ is an order of magnitude more effective than LPC. A similar experiment was done on real speech; both LPC and $B R F$ were done (pitch synehronously) on voiced speech, then noise was added to the speech and LPC and BRF were done again. Recovered pole frequencies and bandwidths vary as noise is added; the expected "error" is less for BRF than for LPC. BRF analysis and resynthesis of speech will be demonstrated by the playing of a short passage.

\section{$10: 40$}

RR11. Adaptive linear prediction filtering for speech analysis. D. L. Heisey (TRW DSSG, Redondo Beach, CA 90278)

An adaptive linear prediction filter utilizing Widrow's leastmean-square noisy gradient algorithm is under study as part of TRW's ongoing voice processing effort. The computational simplicity of the algorithm, as well as its ability to quickly adapt to changes in the statistics of the input speech, makes the algorithm attractive for voice processing. Unlike other block form LPC algorithms which process the data in frames at fixed update rates (autocorrelation and covar iance methods), the filter coefficients are updated at every data sample and a new set of LPC parameters generated at any desired time (variable frame rate). Previous work with the adaptive filter has focused on a cascaded realization for formant analysis [L. B. Jackson and J. Bertrand, Proc. IEEE International Conference on Acoust. Speech Signal Process., pp. 84-86, April, 1976] or input signals other than voiced speech [L.J. Griffiths, IEEE Trans. Acoust. Speech Signal Process. ASSP-23, 207-222 (1975) ]. Preliminary results suggest that the original algorithm as implemented by Griffiths may be unsuitable due to the presence of piteh pulses in voiced speech. A modified version of the algorithm using a fixed step size per iteration appears to minimize this problem resulting in better spectral estimates. Numerical examples which illustrate the properties of the algorithm as compared to other LPC algorithms currently used in speech analysis are included.

$$
10: 50
$$

RR12. The LPC error signal-analysis of its variation with the position of the analysis frame. L.R. Rabiner, B.S. Atal, and M. R. Sambur (Bell Laboratories, Murray Hill, NJ 07974)

The LPC normalized error provides a measure of the success of linear prediction analysis in modeling a speech signal. Very little is known about the variation of the normalized LPC error as a function of the position of the analysis frame. In this talk we show that the LPC normalized error shows a substantial sample-to-sample variation for voiced speech in all three LPC analysis methods-i.e., the covariance method, the autocorrelation method, and the lattice method. The implication of this result is that standard methods of LPC analysis are often inadequate in that the error signal is uniformly sampled at a low rate (on the order of $100 \mathrm{~Hz}$ ) leading to aliased results. For applications such as word recognition with frame-to-frame distance calculations using the normalized error (Itakura, IEEE Trans. Acoust. Speech Signal Process, Feb. (1975) l, the errors due to uniform sampling can be severe. For speech synthesis applications the effect of uniform sampling of the error signal is a small, but noticeable roughness in the synthetic speech. Various strategies for minimizing the aliasing will be discussed.

$$
11: 00
$$

RR13. Speech-quality testing of variable frame rate (VFR) linear predictive (LPC) vocoders, A.W.F. Huggins, R. Viswanathan, and J. Makhoul (Bolt Beranek and Newman Inc. , Cambridge, MA 02138)

VFR transmission of LPC vocoder parameters is a technique developed to reduce the average transmission rate without appreciable loss of quality. The technique transmits parameters at a variable rate in accordance with the changing characteristics of the speech signal. In order to assess the effectiveness of VFR transmission, we performed a pilot experiment to compare it with two other methods for reducing the bit rate: (a) reducing the number of poles, and (b) increasing the quantization step size of the LPC parameters (log area ratios). Thirty-two stimulus sentences were prepared by passing four utterances (two sentences $\times$ two speakers) through elght vocoder systems in a $2 \times 2 \times 2$ factorial design; two values were assigned to each of the three parameters: average frame rate, number of poles, and quantization step size. Eight listeners made seven-point category ratings of quality degradation. The results of the experiment show that, of the three methods studied, the VFR technique produced the highest quality at any given transmission rate. [Work sponsored by ARPA-IPTO.]

\section{$11: 10$}

RR14. The phoneme vocoder: Speech at 160 pps and below. Richard Gillmann (System Development Corporation, Santa Monica, CA 90406)

A computer-based system has been developed which transmits speech at extremely low bit rates, using binary-coded sequences of phonemes. This phoneme vocoder translates digitized speech into phoneme strings using a system developed originally for an automatic speech understanding system. The phoneme strings are then encoded and transmitted to a digital speech synthesizer for reconstruction. Compression to 160 bps has been achieved with the current system. By encoding the phonemes into syllables, rates as low as 38 bps appear to be possible. Results of a study of the accuracy of the phoneme vocoder at the word level will be presented, and a tape recording will be played.

\section{$11: 20$}

RR15. On the design of sub-band coders for low bit rate speech communications, R. E. Croshiere (Bell Laboratories, Murray Hill, NJ 07974)

Digital waveform coding in sub-bands offers attractive possibilities for economically encoding speech at bit rates in the range of $7.2-16 \mathrm{kbits} / \mathrm{sec}$. The design of sub-band coders involves the consideration of a large number of parameters and "tradeoffs." The number of sub-bands, the partitioning of sub-bands (and gaps between sub-bands), allocation of bits among sub-bands and compromises between bits/sample and bandwidth must be considered. In addition, constraints are introduced by practical considerations of integer-band sampling, multiplexing of the digitized sub-band signals, and synchronizing and framing of the data. Design guidelines, 
based on extensive computer simulations and subjeetive com-. parisons, are presented for choosing these parameters and a method for synchronous multiplexing of the sub-band data, without buffering, is proposed. Several examples of sub-band coders for transmission rates of 7.2, 9.6, and $16 \mathrm{Kbits} / \mathrm{sec}$ are presented and compared against that of ADPCM and ADM coders. Tapes will be played to demonstrate the quality of the sub-band coders.

$$
11: 30
$$

RR16. Subjective effects of anomalies in packetized speech transmission. J. W. Forgie (Massachusetts Institue of Technology Lincoln Laboratory, Lexington, MA 0217:3)

When speech is transmitted in a packet-switched network the variability in packet delays inherent in such a net tends to produce occasional anomalies or "glitches" in the output speech when packets fail to arrive at the destination in a timely fashion. While the frequency of occurrence of these anomalies can be minimized at the expense of buffering and increased overall speech delay, it is likely that a practical network design would represent a compromise which allowed some degradation of the output speech under worst case load conditions. To provide some basic data on the subjective effects of such anomalies a series of listening and communicability tests has been undertaken using speech in which anomaly effects have been introduced by simulation techniques. Anomalies resulting from packet losses due to delay dispersion as well as variation in average delay are introduced at controlled rates in simulated networks using a variety of speech encoding teehniques and packetization strategies. Preliminary test results will be presented and a tape with examples of some anomaly effects will be played. [This work was sponsored by the Defense Communications Agency for the Department of Defense. I

$$
11: 40
$$

RR17. Speech encryption by manipulations of LPC parameters. M. R. Sambur and N. S. Jayant (Bell Laboratories, Murray Hill, NJ 07974)

This paper discusses several manipulations of LPC (linear predictive coding) parameters for providing speech encryption. Specifically, the paper considers temporal rearrangement or scrambling of the LPC code sequence, as well as the alternative of perturbing individual samples in the sequence by means of pseudorandom additive or multiplicative noise. The latter approach is believed to have greater encryp- tion potential than the temporal scrarabling technique, in terms of the time needed to "break tre secrecy code." The encryption techniques were assessed on the basis of perceptual experiments, as well as by means of a quantitative assessment of speech-spectrum distortion, as given by an appropriate "distance" measure.

$$
\text { 11: } 50
$$

RR18. Effect of room noise and circuit noise on speech-quality ratings over operator-type headsets ${ }_{\circ}$ Linda Pieree and Michael Pavel (Bell Laboratories, Holmdel, IJJ 07701)

Categorical judgments of communication quality over operator-type headsets were obtained as a function of room noise, circuit noise, and speech volume. The room noise, recorded at an airline reservation center, was slmilar to wide-band pink noise and consisted primarily of voice babble and teletypewriter noise. It was played back in the test room at levels from 55 to $73 \mathrm{dBA}$ with the spectrum matched to that of the original room noise. The circuit noise, ranging from 37 to $76 \mathrm{~dB}$ SPL filtered by a telephone network, extended over a band from 300 to $3300 \mathrm{~Hz}$. The speech was also within this band and ranged in level from 85 to $97 \mathrm{~dB}$ SPL. The stimuli used were $\mathrm{PB}$ sentences and all speech/circuit noise ratios were at high intelligibility levels. The data indicat e that both room noise and circuit noise degrade communication quality ratings. The relative effects of the room noise on the headset ear and the uncovered ear were different for the two headsets compared.

$$
12: 00
$$

RR19. Research in electronic determ!nation of speech intelligibility. E. Schiller (Noise and Commsunication Branch, Naval Electronics Laboratory Center, San Liego, CA 92152)

A simple, fast, accurate and inexpensive device for the evaluation of speech intelligibility of comrnunication equipment and systems is the subject of this investigation. The subjective methods presently used for evaluating intelligibility require extensive training and controls over the testing for accurate predictions of intelligibility, but still show variability between testing labs and are not standardized lor different languages. Therefore, an instrument for evaluating intelligibility cannot be expected to correlate with all subjective tests, but it can produce the same results as all identical instruments under similar conditions any place on earth. This repeatability would be valuable to the Navy and probably to many other organizations. 


\title{
Session SS. Musical Acoustics III and Committee on Education in Acoustics I: Teaching the Physics of Sound I
}

\author{
Diana Deutsch, Chairperson \\ Center for Human Information Processing, University of California, San Diego, \\ La Jolla, California 92037
}

Invited Papers

9:00

SS1. Pedagogical aspects of teaching acoustics to a general student group. William $R$, Savage (Department of Physics and Astronomy, The University of Iowa, Iowa City, LA 52242)

At a number of colleges and universities courses in introductory acousties and related areas are being offered. Often these courses are taught in the physics or physical science departments as a science subject. This is not a universal rule since similar courses are found in departments such as speech science, architecture, etc. The teacher may have a deep interest in acoustical phenomena without being a professional acoustician. The courses may serve as a required course for students in the speech sciences or music. These courses can have a large enrollment when they are open to general interest students. Many of the teaching methods relate to the task of explaining a phenomenon that is described perhaps best, at least compactly, by mathematical methods with visual or audible teaching aids.

$9: 15$

SS2. Music, speech, and hearing: A general education course in descriptive acoustics, W. J, Strong. E. P. Palmer, and J.D. Dudley (Department of Physics and Astronomy, Brigham Young University, Provo, UT 84602)

A general education course in descriptive acoustics for students in music, speech, and related areas is described. Resource materials include books, study guide, films, audio tapes, demonstrations, and walk-in labs. The course has been evolving as it has been offered over the past 16 semesters. However, our university has recently adopted a general education program which allows students to test out on a course without formally enrolling. This has necessitated making the exams and resource material more generally available, and poses a particular challenge for lab-oriented activity. Much of this paper is concerned with aspects of a walk-in hands-on lab designed to meet these new requirements,

\section{$9: 45$}

sS3. Teaching psychoacoustics to a mixed audience of music, science, and humanities majors. Juan G. Roederer (University of Denver, Denver, CO 80208)

Boundary conditions imposed by widely different student backgrounds in an elementary course on musical psychoacoustics demand careful identification of relevant topics and formulation of questions that are attractive and comprehensible to all sectors involved. [J.G. Roederer, Introduction to the Physics and Psychophysics of Music, 2nd ed. (Springer, New York, 1975)l. The following topics and related questions have been found suitable for treatment in such a course: (a) Perception of spectral pitch of sinusoidal tones (Why do we have good pitch resolution in spite of poor cochlear frequency analysis?), (2) Perception of subjective pitch of complex tones (Why do we perceive tones made up of many harmonics as single-pitched wholes? Why do we perceive a missing fundamental?), (3) Consonance, dissonance, and tonic dominance (Why do we call all notes that are octaves apart by the same name? Is harmony the result of a learning process?), and (4) Hemispheric specialization of speech and music processing in the brain (Which tasks of music perception involve preferentially one cerebral hemisphere? To what extent do language centers of the brain become involved in musical information processing?) The talk will focus on the evaluation of teaching experience in class and laboratory covering the above topics.

$$
10: 15
$$

SS4. Electronic demonstrations for the teaching of acoustics, W. M. Hartmann (Physics Department, Michigan State University, East Lansing, MI 48824)

Courses in elementary acoustics taught at many universities provide introductions to the following six topics : physical acoustics, environmental acousties, the physiology of hearing, auditory psychophysics, musical acoustics, and the nature of human speech. Electronic demonstrations can be used to enhance instruction in each of the six areas. For example, the modules of an electronic music synthesizer together with an audio system and conventional electronic instrumentation form a versatile laboratory for experiments in sound. Among the demonstrations which can be done are the following. Wave interference can be demonstrated 
by extracting the separate signals from a ster eo recording of signals $\mathrm{A}+\mathrm{B}$ and $\mathrm{A}-\mathrm{B}$. Tone and noise bursts can be used to find the speed of sound, echo and reverberation times. Filter banks, set according to mean audiometric presbycusis data, can show the effects of ak,ing on the frequency response of human hearing. Gently nonlinear electronic devices aid $t$.1e recognition of combination tones nor mally generated by the inner ear. Analog delay lines can create repetition pitch from a source of white noise. Sine wave signals from many oscillators can be summed and varied to study the perception of pitch of an inharmonic tone complex like that of a chime. The phase variation of a single rectangular wave with pseudorandom pulse width modulation simulates chorus effect. Much of the effect of nonlinear mode nnixing in conventional musical instruments can be simulated by patched of linear electronic components, transient generators and voltage controlled signal processing modules. Three resonant filters allow the synthesis of vowel sounds. Frequency shifted or ring modulated speech sounds can exhibit the importance of vowel formant transitions. Intelligibility experiments with chopped speech serve as an introduction to redundancy, autocorrelation and information theory. We note that one does not pursue this informal experimenting with signal processing equipment for long before one discovers effects which are hard to understand. Further pursuit can lead to new results. For example, our intelligibility experiments with chopped speech suggest that redundancy measured in spoken English text agrees with that found by Shannon for written text.

$$
10: 45
$$

SS5. Keller Plan applied to instruction in acoustics, Michael R. Chial (Department of Audiology and Speech Sciences, Michigan State University, East Lansing, MI 48824)

Undergraduate students in audiology, speech pathology, and education of the deaf present two problems for the would-be teacher of acoustics. First, they are typically deficient in basic mathematics and physics. As a result, they fall victim to "symbol shock," a disorder characterized by glazed eyes and cortical dysfunction. Second, they require at least a modest understanding of acoustics for their later professional health. A reasonable treatment has been found in the form of the Keller Plan or the Personalized System of Instruction (PSDD. This plan has been used successfully for four years in an undergraduate course designed to convey the rudiments of physical acoustics, bioacoustics, and psychoacoustics. PSI uses explicit performance criteria, unit mastery, self-pacing, and individualized tutoring. Laboratory demonstrations are used for motivational purposes and to promote student discovery. Critical information is conveyed by the written word; lectures are given only upon student request. Details of the PSI method will be presented, as well as the results of a modest retrospective analysis of the course taught under traditional and PSI formats.

$$
11: 15
$$

SS6. Project laboratory for a course on the physics of music. John I. Shonle (Department of Physics, University of Colorado at Denver, Denver, CO 80202)

The spectrum of laboratory styles ranges from a different exercise every week, complete with "cookbook" instructions, to research projects. The advantages of the latter style include high student enthusiasm, a better understanding of the methods of experimentation, and a much deeper knowledge of the chosen area. Our students, usually working in pairs, investigate a single area of acoustics for the semester. Topics are chosen by the students in consultation with the instructor. Examples of topics which have been undertaken in the last four years and method of organization will be given.

\title{
Session TT. Noise VII: Measurement and Reduction of Aircraft Noise Sources
}

\author{
Alan H. Marsh, Chairperson \\ Dytec Engineering, Inc., Huntington Beach, California 92649
}

Invited Papers

$9: 00$

TT1. The remotely operated multiple array acoustic range (ROMAAR) for the direct measurement of airplane flyover noise footprints. D. A. Hilton and H. R. Henderson (NASA-Langley Research Center, Hampton, VA 23665)

The ROMAAR now in operation at NASA will allow direct measurement and display of aircraft noise contours in several measurement units during takeoff, landing, and flyby opirations. Direct measur ement of contours allows determination of the statistical variation of contours due to the atmosphere or aircraft operational parameters, a measure of the impact 
of various noise reduction techniques and hardware on ground noise footprints, and the ability to supply direct inputs for improvement of the NASA Aircraft Noise Prediction Office predictive program. The methods, techniques, and equipment developed for the ROMAAR concept are applicable to CTOL, STOL, General Aviation, and VTOL aircraft. ROMAAR represents a unique combination of state of the art digital and analog noi se recording methods, computercontrolled digital communications methods, radar-tracking facilities, quick look weather capabilities, and a large data handling facility complemented by a large capacity curve fitting and plotting routine. The ROMAAR is set apart from the standard airport noise monitoring system by having the unique features mentioned above plus the fact that up to 33 separate (but simultaneous) noise measurements are made for each aircraft overflight. This paper will present a description of the ROMAAR facility and its uses along with actual measured and predicted noise footprints.

\section{Contributed Papers}

\section{$9: 30$}

TT2. Measuring atmospheric temperature and humidity for aircraft flyover noise testing. C. R. Hogstedt (Flight and Laboratory Development, Douglas Aircraft Company, Long Beach, CA 90846)

The measured of near-surface and upper-air temperature and humidity is required during flyover noise tests to adjust the noise data to reference-weather conditions or to classify the data with respect to various criteria. Parallel advancements in equipment, test techniques, and noise data adjustments have occurred in the last several years at the Douglas Aircraft Company. Measurements of near-surface temperature and humidity began with a psychrometer hand-held at four feet above the ground and subsequently evolved through a series of instrument types mounted 33 feet above the ground on a trailered tower. Upper-air (or sound-path) weather data were initially acquired from local weather bur eaus and later from various instrumented light aircraft. The more notable improvements are the use of dew-point sensors for humidity measurements, increased data sample rates, and system performance monitoring. Current instruments, along with sufficient attention to time and space dependencies, appear to furnish an adequate definition of the test atmosphere. However, more data are required to quantify and evaluate the actual accuracy of the system.

$$
9: 45
$$

TT3. Modal density function and number of propagating modes in ducts. E. J. Rice (NASA-Lewis Research Center, 21000 Brookpark Rd., Cleveland, $\mathrm{OH} 44135$ )

Often raised questions in duct sound propagation studies involve the total number of propagating modes, the number of propagating radial modes for a particular spinning lobe number, and the number of modes possible between two given values of cutoff ratio or eigenvalue. These questions can be answered approximately by using the modal density function for ducts in a manner similar to that previously published for architectural acoustics. The modal density functions are given for rectangular and circular ducts with a uniform steady flow. Results from this continuous theory are compared to the actual (discrete) modal distributions.

$$
10: 00
$$

TT4. Source array for generating higher order acoustic modes in circular ducts. B. R. Wyerman (Owens-Corning Fiberglas, Technical Center, Granville, OH 43023) and Dr. G. Reethof ('The Pennsylvania State University, University Park, PA 16802)

A unique source array has been developed for the generation of both spinning and nonspinning higher-order modes in a circular duct. The array consists of two concentric rings of sources. Through individual control of the response of each element, the array provided phase and amplitude control in the radial as well as circumferential directions. Radial mocies shapes were measured in a 12-in. diameter anechoically-terminated hollow duct. These modes could be generated at their cut-off frequency and throughout a frequency range extending to the cut-off frequency for the next higher-order radial mode. Comparisons are given between theory and experiment for the generation of specific modes. The radial dependence of the measured mode shapes was enhanced considerably by the design of this array. The results indicate a significant improvement over previous mode generation mechanisms. The contamination of the generated mode by additional spurious modes is also considered for variations between individual elements within the source array. (Work supported by NASA Langley Research Center. $]$

\section{$10: 15$}

TT5. Sound attenuation in acoustically lined curved ducts in the absence of fluid flow. Sung-Hwan Ko (New London Laboratory, Naval Underwater Systems Center, New London, CT 06320) and Louis T. Ho (David Taylor Naval Ship Research and Development Center, Annapolis, MD 20084)

A study has been made of the sound attenuation in a lined curved duct with rectangular cross section. In this study, the derivation of eigenvalue equation was based on the continuity of the normal component of the particle displacement and the matching of the acoustic pressure on the acoustic lining surface. The sound attenuation was calculated using the acoustic energy expression for the waves propagating in a curve duct. For a given duct geometry and known acoustic lining impedances, a computer program was developed to solve for the eigenvalues and to obtain the sound attenuation of the propagating waves in the lined curved duct. It was found that the fundamental mode was least attenuated. The total sound attenuation was calculated assuming that the amplitudes for all propagating waves were equal at a given frequency. Effects of aspect ratio, bent angle, and the acoustic impedance on the sound attenuation were investigated in the present work. [Work sponsored in part by Naval Sea Systems Command Code 037.]

$$
10: 30
$$

TT6. Core noise source diagnostics on a turbofan engine using correlation and coherence techniques. Allen Karchmer and Meyer Reshotko (NASA-Lewis Resear ch Center, Cleveland, $\mathrm{OH} 44135$ )

A test program was conducted on a Lycoming YF-102 engine to identify sources of core noise. Specially constructed "semiinfinite" waveguide microphone probes were installed at various locations within the combustor and core nozzle. The pressure signals from the core nozzle resulted in the strongest correlation with microphone signals in the acoustic farfield. Between the combustor pressure signal and that of the farfield the shape of the cross-correlation function indicates a second time derivative relationship. This suggests that the combustion region is, in fact, a source region for core noise radiating to the farfield. This conclusion is drawn from a simplified model of Lighthill's acoustic analogy which indicates that there can be an acoustic contribution from a combustion region that is related to the second time derivative of the fluctuating entropy. Measurements of the pressure coherence function between various internal locations and the farfield appear 
to indicate the combustion contribution to the farfield noise from the YF-102 engine is confined to frexuencies less than about $200 \mathrm{~Hz}$.

\section{$10: 45$}

TT7. Flight effects on exhaust noise for turbojet and turbofan engines-comparison of experimental data with prediction. James R. Stone (NASA-Lewis Research Center, Cleveland, OH 44135)

Recent experiments on the effects of flight on jet engine exhaust noise have produced apparently conflicting results. Some of these results do not agree with projections based on classical jet noise theories nor with experimental results from model jet simulated flight tests. It has been shown that in some of the cases reported, the proper corrections were not made to account for the distributed nature of the jet noise sources. It is shown herein that the remaining discrepancies can be reconciled by considering the combined effects of jet-mixing noise, internally generated engine exhaust noise, and shock noise. This paper demonstrates that static and in-flight jet engine exhaust noise can be predicted with reasonable accuracy when the multiple-source nature of the problem is taken into account. Jet mixing noise and shock noise are predicted from an improved version of the NASA inter'im prediction method. A provisional method of estimating internally generated noise is used, based partly on existing prediction methods and partly on recently reported engine data.

$$
\text { 11:00 }
$$

TT8. Effects of forward velocity on noise for a J85 turbojet engine with multi-tube suppressor from wind tunnel and flight tests, J. R. Stone, J. H. Miles, and N. B. Sargent (NASALewis Research Center, Cleveland, OH 44135)

In order to obtain an understanding of the effects of forward velocity on exhaust noise suppression, flight and wind-tunnel noise tests were conducted using a J85 turbojet engine. Nozzle configurations of primary interest were a 104-tube suppressor with and without an acoustically treated shroud. Engine installation was as nearly idential as possible for both flight and wind tunnel tests. Some differences necessarily existed, however; e.g. , for the flight tests the engine was in motion with respect to the microphones, and for the wind-tunnel tests it was not. Exact simultaneous matching of engine speed, exhaust velocity, and exhaust temperature was not possible, and the wind-tunnel maximum Mach number was $\sim 0.27$, while the flight Mach number was $\sim 0.37$. The nominal jet velocity range was $450-$ $640 \mathrm{~m} / \mathrm{sec}$. For both experiments, background noise limited the jet velocity range for which significant data could be obtained. The observed forward velocity effects for the suppressor indicate that jet noise has been suppressed to a level where the in-flight directivity is similar to that expected for internally generated noise.

\section{$11: 15$}

TT9. Simulation of flight-type engine fan noise in the NASALewis $9 \times 15$ anechoic wind tunnel. M. F. Heidmann and D. A. Dietrich (NASA-Lewis Research Center, 21000 Brookpark Rd. , Cleveland, $\mathrm{OH}$ 44135)

A major problem in the measurement of aircraft engine fan noise is the difficulty in simulating in a ground-based facility the noise that occurs during flight. Flight-type noise as contrasted to the usual ground-static noise exhibits substantial reductions in (1) the time unsteadiness of tone noise and (2) the mean level of tones calculated to be nonpropagating or cutoff. A model fan designed to have a nonpropagating tone was acoustically tested in the anechoic wind tunnel under both static and tunnel flow conditions. The properties that characterize flight-type noise were progressively simulated with increasing tunnel flow. The distinctive lobed directivity patterns of propagating duct modes were also observed. The acoustical data with tunnel flow appear uncontaminated by the excess noise attributed to the ingestion of flo $x$ disturbances that prevail near most static test facilities. This anechoic wind tunnel appears to be a useful facility for flig tt applied acoustic research on aircraft engine fans.

$$
11: 30
$$

TT10. Attenuation of jet noise in a lined augmenter tube: A model study. István L. Vér and Douglas W. Andersen (Bolt Beranek and Newman Inc., 50 Moultorı St. , Cambridge, MA 02138) and James L. Grunnet (Flui Dyre Engineering Corporation, Minneapolis, MN 54422)

To obtain needed information for the design of an air-cooled jet aircraft run up noise suppressor system, scale-model investigations have been carried out. Acoustical, thermal, and pumping performances of various augmenter tube configurations, utilizing a jet exiting from a 2,5 -in. -diam. nozzle as a function of pressure ratio (up to ?) and temperature (up to $3300^{\circ} \mathrm{F}$ ), have been investigated. Base data, obtained with hard-walled augmenters, have been already described in an earlier paper [I. L. Vér, D. W. Anderssen, and J. L. Grunnet, J. Acoust. Soc. Am. 59, S95(A) (1976']. This paper reports on the measured attenuation of the jet ooise in lined augmenter tubes of various constructions as functions of jet temperature, pressure ratio, augmenter tube length, and frequency. The effects of noise regeneration at the exit plane, pumping performance, and the temperature and flow velocity distribution will also be discussed. [This work was supported, in part, by the U.S. Navy, Southern Division, Naval Facilities Engineer ing Command. 1

$$
11: 45
$$

TT11. Macroscopic study of time unstendy noise of an aircraft engine during static tests. B.J. Clark, M. F. Heidmann, and W.J. Kreim (NASA-Lewis Research Center, 21000 Brookpark Rd., Cleveland, $\mathrm{OH}$ 44135)

Static tests of aircraft engines can exhibit greater than 10$\mathrm{dB}$ random unsteadiness of tone noise '.evels because flow disturbances that prevail near test site facilities are ingested. Presumably such changes are related to installation and test site features. This paper presents some properties of unsteady noise observed at a NASA-Lewis facility during tests of a Lycoming YF-102 turbofan engine. Tiree and spacial variations in tone noise obtained from closely spaced farfield and inlet duct microphones are displayed. Long $(0.5-\mathrm{sec})$ to extremely short (0.001-sec) intermittent tone bursts are observed. Unsteadiness of the tone, its harmonics, and the broadband noise show little similarity. In the farfield, identity of tone bursts is retained over a dir ectivity angle of less than $10^{\circ}$. In the inlet duct, tone bursts appear to propagate axially but exhibit little circumferential similarity. They show only slight relationship to tone bursts observed in the farfield. The results imply an intermittent generation of random mixtures of propagating duct modes. 


\title{
Session UU. Physical Acoustics VII: Atmospheric Acoustics/Physical Acoustics
}

\author{
Paul B. MacCready, Jr., Chairperson
}

Aero Vironment, Inc., Pasadena, California 91107

\author{
Contributed Papers
}

$8: 30$

UU1. Study of the behavior of radiational inversions with a calibea ddacoustic sounder, B. R. Kerman, H. E. Turner, R. E Tek $\sigma$ and W. Clink (Atmospheric Environment Ser-

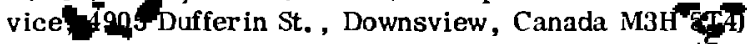

A calibrated acoustic sounder developed by the Atpospheric Environment Service of Cenada is compared to an in situ sensor measuring the acoustic backscatter cross-section directly, Some deficiencies in the calibration technique and disfrepancies with the in situ sensor are analyzed. The vertice structure of the acoustic backscatter cross-section duning 4 ious stages of a radiational inversion is examined in conjui, fion with supporting temperature, humidity and velocity data taken from a tethbed balloon system.

$$
8: 45
$$

UU2. Antennas for sodar systems. U. I. Kurze (BattelleInstitut e. .. , Frankfurt/Main, Germany)

Theirento probing of the thermal structure and the wind

ii. velocity in the lower atmosnhere requires highly directive antennas, which should have a half-power beam width of about $\pm 6^{\circ}$ (3-dB points), a $90^{\circ}$-side-lobe suppression of at least $40 \mathrm{~dB}$, little volume reverberation, low wind sensitivity, and be made of weather-proof material, Various off-axis parabolic horn antennas have been built and acoustically evaluated, considering additional requirements of mobility. The performance of shielding tubes and their absorbent lining, the transmission loss of the antenna walls and the influence of antennas of different size have been evaluated both theoretically and experimentally. Detailed studies of antenna elements are supplemented by field applications of sodar systems.

\section{- 9:00}

UU3. Observation of upper atmosphere warmings with the use of natural infrasound. D. Rind and W. L. Donn (LamontDoherty Geological Observatory of Columbia University, Palisades, NY 10964)

Natural infrasound of 5 sec period is generated by interfering ocean waves in the Atlantic Ocean to the east of our location. Our normal amplitude pattern of infrasound in winter is characterized by semidiurnal and diurnal oscillations associated with reflecting tidal winds in the lower thermosphere $[W . L$. Donn and D, Rind, J, Atmos, Sci, 29, 156-172 (1972); D. Rind and W. Donn, J. Atmos. Sci. 32, 1694-1704 (1975) ]. Occasionally an amplitude pattern featuring continuously strong signal throu pout the day is observed. Eight of these cd bes were investisted and it is shown, with the use of obseirvations at Wallops Island, that seven of these coincide with observed stratospheric warmings during which east winds . replace west winds, allowing for reflection of signal from the east at $40-50 \mathrm{~km}$. The continuously high amplitudes result from reflection in the stratosphere where strong dissipation and tidal winds are minimal. Conversely when stratospheric westerlies diappear at times of warmings, infrasound from the Pacific diminishes at western stations. Continuous highamplitude patterns not associated with an observed upper air warming are discussed as perhaps signifying unnoticed mesophere and lower thermosphere warmings.
$9: 15$

UU4. Leaking modes in the propagation of atmospheric acoustic-gravity waves. W. A. Kinney and A. D. Pierce (Code 8160, Naval Research Laboratory, Washington, Dg 20375)

Attempts to predict the transient acoustic pressure pulse at long horizontal distances from large explosions in the atmosphere have adopted a model atmosphere bounded above by a halfspace of finite sound speed and have represented the waveform as a superposition of contributions from dispersively propagating guided modes. Certain modes at low-frequency decay exponentially (leaking modes) with increasing distance, of propagation. Until now, the practice has been to neglect the contributions from leaking modes $a$ low frequency. The theory associated with leaking modes at low fripuency. The theory associater with leaking modes has been previously discussed in detail th Al Kinney, A, D. Pierce, and C.Y. Kapper, J. Acoust. Soc. Am. 58, S108(A) (1975) l. Examples of numerically synthesized transient waveforms are now exhibited with and without the inclusion of leaking modes. The inclusion of leaking modes results in waveforms each having-frarked beginning rather than a low-frequency oscillating precursor of gradually increasing amplitude as previously obtained. Also, the revised computations indicate that yoforms invariably begin with a pressure rise; a result suptoted by other theoretical considerations and experimental data. [Work supported by Air Force Geophysics Laboratory.

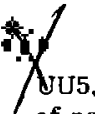

Uu5. Upper atmosphere circulations as observed with the use of natural infrasound. D. Rind (Lamont-Doherty Geological Observatory of Columbia University, Palisades, NY 10964)

Natural infrasound of $5-\mathrm{sec}$ periods is generated continuously by ocean waves to the east of our location. The amplitude characteristics and horizontal phase velocity are functions of the acousistic reflection characteristics of the upper atmosphere. The monthly patterns of the observed acoustic parameters, culled from eight years of continuous observations, are reviewed and compared to the existing knowledge of $30-120-\mathrm{km}$ winds and temperatures. Included in the discussion are (1) the percentages of observed semidiurnal and diurnal amplitude oscillations for each month, related to the reliability of these tides in the upper atmosphere; (2) annually repeating oddities in each month such as a late March increase in semidiurnal tidal strength and varying meridional wind influences from summer to winter; and (3) the failure of current atmospheric models to correctly predict the observed lower thermosphere sound velocity at 0400 which is much greater from the east then predicted.

\section{$9: 45$}

UU6. Sound reflection from locally reacting surfaces of finite extent, W. James Hadden, 1., Robin A. Vidimos, and Philip M. Sencil (Sehoolef Mfthanical Engineering, Georgia Institute of Technology, Alanta, GA 30332)

An approximation for the scattering of spherical waves by a rectangular patch with finite acoustic impedance is presented.

: Comparisons are made with laboratory experiments in which sound pressure levels were measured in the direction of specular reflection. Particular attention is given to the way in 
which both the theoretical and experimental results approach the limiting case of a plane surface with finite acoustic impedance.

\section{$10: 00$}

UU7. Acoustic/seismic coupling phenomenology. J.G. Constantine and R. B. Walker (Eglin AFB, FL 32542)

This paper summarizes the results of a recent experiment that was designed to characterize the signal generation and propagation media in order to develop an acoustic/seismic signal propagation model. Specifically, this paper discusses the coupling of acoustic energy in the soil as a function of frequency; the observation of signal amplitude variations as a function of distance and frequency; and the measurement of signal distortion through the calculation of the normalized correlation and coher ence function. It has been found that acoustic/seismic cross properties provide good discrimination par ameters and that seismic and acoustic differential propagation velocity and seismic multimode propagation velocity can be used to provide ranging information; that the signal amplitude Is a direct function of the soil coupling properties and soil inhomogeneities; and that the acoustic/seismic, as well as the seismic propagation channel, is nonlinear and can be best character ized through the application of Weiner's nonlinear theory.

\section{$10: 15$}

UU8. Radiation in a random medium. Alan $R$. Wenzel (ICASE, NASA-Langley Research Center, Hampton, VA 23665)

A theoretical investigation of the wave field radiated by a point source in a random medium is described. The analysis, which is based on the smoothing method, deals specifically with time-harmonic waves in a medium which is statistically homogeneous and isotropic. Approximate results are obtained for the special case in which the wavelength is much less than the correlation length of the medium. These results show that, In a mean-square sense, the randomness of the medium leads to a slight amplification of the wave field. [Work supported by NASA. 1

\section{0:30}

UU9. Acoustically induced instabilities of control valves. Uno Ingard (Research Laboratory of Electronics, Massachusetts Institute of Technology, Cambridge, MA 02139)

Recent theoretical and experimental studies of acoustically induced axial oscillations of control valves are reviewed, and a mechanism for acoustically induced lateral oscillations is proposed. Possible methods of quenching the instabilities are also diseussed. [Work supported by ONR.]

\section{$10: 45$}

UU10. Mechanical response of the lung at high frequency. J. J. Fredberg and A. Hoening (Cambr idge Collaborative, Inc. , 238 Main St. , Cambridge, MA 02142)

For the purposes of learning about pulmonary structure, interpretation of high frequency impedance of branching airway systems may be facilitated by a theoretical prediction of input impedance. We have developed ways of treating special classes of asymmetry in branching structures which simulate a variety of observations unaccounted for in current symmetric models. This self-consistent analysis, which assumes that small sub- units can be joined in an appropriate manner to simulate everlarger branching structures, also incorporates the geometric and dynamic properties of the airway walls and enclosed gas. This analysis has been successfully applied to models of adult and infant lungs, and mirrors trends found in existing data. The model also suggests quantitative and qualitative dependences of system impedance responses to certain airway system characteristics, such as wall properties, branching asymmetry, and central and peripheral airway obstructions. Modal overlap and clumping are observed. Wall properties and branching asymmetry are both found to be important at low frequencies $(\leqslant 1 \mathrm{kHz})$, while the asyrmetric nature of the structure predominates at higher frequences $(\geq 3 \mathrm{kHz})$. The effect of central airway obstructions is most marked at higher frequencies $(>2 \mathrm{kHz})$ where the number of appar ent modes has decreased as modal regularity increases. Peripheral airway obstructions, in contrast, have small effect at high frequency.

11:00

UU11. Acoustic nonspecular scattering from a plate with line impedance discontinuities. Peter R. sitephanishen (Department of Ocean Engineering, University of Fhode Island, Kingston, RI 02881)

An approach is presented to investijgate the acoustic nonspecular bistatic scattering of an incident acoustic plane wave by a fluid loaded plate with line impedance discontinuities. The approach is based on the extension of a method which was presented in an earlier paper IP. R. Stepanishen, J. Acoust. Soc. Am. 59, S32(A) (1976) ] to investigate the effect of a single discontinuity on the acoustic transmission and scattering from a plate. The total scattered fleld is expressed as a sum of a specular reflected plane wave and a sum of scattered cylindrical waves which originate at the discontinuities on the plate which is described by Timoshenko-Mindlin plate theory. The scattering strengths of the cylindrical waves can exhibit highly directional characteristics and are expressed, using Fourier transform methods, as the solution of a linear set of coupled equations. Several special cases are investigated and discussed. In particular, it is noted that when the effects of the acoustic-elastic interactions between the scatterers or impedance discontinuities can be neglected, the nonspecular scattered field can be expressed as a jroduct of two terms, i. e. , the nonspecular scattered pressire from a single discontinuity times an equivalent scattering array factor.

\section{1:15}

UU12. Edge mode radiation due to a flu id-loading discontinuity. M. Pierucci (General Dynamics/Electric Boat Division, Advanced Engineer ing Department 440, Groton, CT 06340)

Edge mode radiation is determined for an infinite membrane with a discontinuous fluid loading condition. The membrane is assumed to be line driven. The lower portion of the membrane $(y<0)$ is exposed to a very light fluid which imposes no fluid loading on the structure. The upper half of the membrane $(y>0)$ is split up into two regimes $(x<0$ and $x>0)$. The $x<0$ regimes imposes fluid loading conditions on the membrane vibration while the $x>0$ region is assumed to be a vacuum. The two upper regimes are separated by an acoustic anechoic surface. The radiated acoustic intensity as a function of frequency for differ ent surface tensions and angular positions is derived and results are presented. [Portion of this work was sponsored by NAVSEA.] 

$2: 45$

VV6. On the growth of the pulsation threshold of a suppressed tone, Robert V. Shannon (Institute for Perception, Soesterberg, Nether lands)

Consider a $1200-\mathrm{Hz}$ tone suppressing a $1000-\mathrm{Hz}$ tone. For a fixed level of the $1200-\mathrm{Hz}$ tone $(50 \mathrm{~dB})$ the pulsation threshold was measured at $1000 \mathrm{~Hz}$ as the $1000-\mathrm{Hz}$ tone in the "masker" was raised from absolute threshold to $60 \mathrm{~dB}$ in 5$\mathrm{dB}$ steps. When the level of the $1000-\mathrm{Hz}$ tone was less than the simultaneous masked threshold at $1000 \mathrm{~Hz}$ the pulsation threshold measured was the same as if the $1000-\mathrm{Hz}$ tone were not present in the "masker". When the level of the $1000-\mathrm{Hz}$ tone was raised above the simultaneous masked-threshold level the pulsation-threshold level increased at the same rate as the physical level-maintaining a constant number of decibels of suppression. When the level of the $1000-\mathrm{Hz}$ tone was raised above the level of the $1200-\mathrm{Hz}$ tone the pulsation-threshold level increased faster than the physical level, showing "recruitment" of pulsation threshold or a reduction in suppression. [Work supported by NINCDS. I

$$
3: 00
$$

VV7. Suppression in pulsation-threshold patterns, Robert V. Shannon (Institute for Perception, Soester-berg, Netherlands)

In a pulsation, threshold experiment the frequencies and intensities of the two tones in the "masker" were fixed and the frequency of the signal was varied. The plot of pulsation threshold versus signal frequency is called the pulsationthreshold pattern of the "masker". First the pulsation-threshold pattern was measured when the "masker" contained only a $1000-\mathrm{Hz}$ tone. Then the patterns were measured for a $400-\mathrm{Hz}$ tone and for a $1500-\mathrm{Hz}$ tone. Finally, the pattern was measured when the masker contained both the $1000-\mathrm{Hz}$ tone and either the $400-\mathrm{Hz}$ tone or the $1500-\mathrm{Hz}$ tone. The $400-\mathrm{Hz}$ tone was able to reduce the pulsation thresholds around $1000-\mathrm{Hz}$. by $20 \mathrm{~dB}$ or more for each of the three subjects. The $1500-\mathrm{Hz}$ tone did not reduce the pulsation thresholds at $1000 \mathrm{~Hz}$ but did

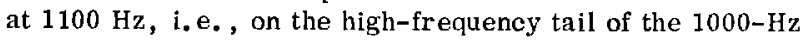
pattern. Suppression is assumed to be the cause of these reductions. Thus one tone is able to suppress only part of the excitation pattern of another tone. Mutual suppression was also indicated for one subject. Relationships to partial masking will be discussed. [Work supported the NINCDS.]

\section{$3: 15$}

VV8. Measurement of a binaural temporal transfer function. D. W. Grantham and F. L. Wightman (Auditory Research Laboratory, Northwestern University, Evanston, IL 60201)

In order to derive a kind of "binaural temporal transfer function" for the auditory system, observers detected a stable target (a binaural click presented with an interaural time difference of $+500 \mu \mathrm{sec}$ ) imbedded in a "moving" masker (a noise presented with an interaural delay sinusoidally varied from +500 to $-500 \mu \mathrm{sec})$. Detectability of the click was measured as a function of the "position" of the noise at the time the click was presented and the rate at which the noise "moved". As was expected, when the noise moved very slowly, less than $1 \mathrm{~Hz}$, or not at all, detectability was best when the click appeared at one ear and the noise appeared at the other, and poorest when they both appeared at the same ear. At high rates $(5 \mathrm{~Hz})$ detectability was independent of the relative positions of the click and the noise. [This work was supported in part by NIH Grant NS12045.]

$$
3: 30
$$

VV9. Modulation thresholds and temporal modulation transfer functions, Neal F. Viemeister (Department of Psychology, University of Minnesota, Minneapolis, MN 55455)

Temporal Modulation Transfer Functions (TMTF's) were obtained by measuring the threshold amplitude of sinusoidal modulation as a function of modulating frequency. For modulation frequencies below approximately $800 \mathrm{~Hz}$, TMTF's ob- tained with a continuous wide-band noise carrier generally show the low-pass characteristic reported previously [J. Acoust. Soc. Am. 53, 314(A) (1973)]. That is, with increasing modulation frequency the amplitude of modulation required for threshold remains constant up to approximately $10 \mathrm{~Hz}$ and then increases monotonically up to $810 \mathrm{~Hz}$. The interpretation is that at high modulation frequencies the auditory system temporally "smooths" the amplitude fluctuations produced by modulation and the observer therefore requires greater modulation amplitude at the input in order to detect the modulation. For modulation frequencies greater than $300 \mathrm{~Hz}$, modulation threshold is independent of modulation frequency and ean be predicted from the increment threshold for wide-band noise. The form of the empirical TMTF generally agrees with that predicted by the familiar model consisting of half-wave rectification followed by "leaky integration." The time constant of the integrator is estimated to be $3 \mathrm{msec}$. [Research supported by NIH. I

$$
3: 45
$$

VV10. Structural rules for the perception of the periodicity of repeated random waveforms, Irwin Pollack (Mental Health Research Institute, The University of Michigan, Ann Arbor, MI 48109)

Two structural rules underlying the periodicity of repeated random wavefor $m s$ were examined: the number of successive copies of the to-be-repeated elements; ; and phase inversion of the to-be-repeated elements. Two behavioral measures of periodicity strength were defined: the accuracy of pitch matching and the fraction of independently varying elements which must be inserted with a repeated random pattern for a threshold level of discrimination from a nonrandom pattern. A variety of autocorrelation measures were performed upon the signals. The signal measure most closely related to behavioral measures of periodicity strength is the maximum autocorrelation among the first 20 periodic deliy intervals. Additional weightings with the average autocorrelation across the 20 delay intervals failed to improve further the relation with periodicity strength.

\section{$4: 00$}

VV11. Pitch strength of ripple noise. William A. Yost and Richard Hill (Institute for the Advaneed Study of Communication Processes and Psychology Department, University of FIorida, Gainesville, FL 32601)

Ripple noise is produced when a broadband noise is delayed $(T)$ and either added to or subtracted from the original noise. The resulting waveform has a power spectrum in which power varies cosinusoidally as a function of frequency. Ripple noise has been used to study pitch percepticn and echo processing. In the present experiments subjects were asked to discriminate between two noises which differed in the amount of delay introduced (one stimulus continued a delay $T$ the second stimulus a delay $T+0.09 T)$. The discriminability of these two noises was studied as a function of the amount of attenuation added to the delayed noise. Discriminations between these two noises could be made when $T$ rangred from 0.5 to 20 msec before the subjects could no lon;er make the discrimindelayed noise when the delays were in the range from 2 to 5 $\mathrm{msec}$ before the subje cts could no longer make the discriminations. More attenuation could be added to the delayed noise for threshold discrimination when it was added back to the original source than when it was subtrac:ed from the original source than when it was subtracted from the original source. Discrimination did not change as a furiction of octave band filtering the ripple noise when the center frequency of the filters varied from 200 to $3200 \mathrm{~Hz}$. The results will be discussed in terms of pitch perception, especially in regard to the concept of a spectral dominance region for pitch and pitch strength. The data will also be related to the echo processing abilities of the auditory system. [Work supported by NSF.] 
$4: 15$

VV12. Detection of signals with identical energy spectra but different pitches under conditions of signal uncertainty. C. Lundeen and A. M. Small, Jr. (Department of Speech Pathology and Audiology, University of lowa, Iowa City, IA 52240)

Previous research has shown that a signal which is one of two pure tones will be less detectable than a signal that is of constant, known frequency throughout. Similar results were obtained in the present study using pulse train signals with identical energy spectra but different pitches. This finding cannot be attributed to the "mistuned" critical-band mechanism postulated in the single-band $[W . P$. Tanner Jr. and R. Z. Norman, IRE Trans. Prof. Group Inf. Theory PGIT-4, 222227 (1954)] and multiple-band [D. M. Green, J. Acoust. Soc. Am. 30, 904-911 (1958) $]$ models of selective auditory attention. Our subjects performed as if they could selectively attend to phase, and/or periodicity pitch cues in order to optimize the detectability of anticipated signals.

$$
4: 30
$$

VV13. Dichotic perception of virtual pitch: Dependence on subjective lateral position of partials. W. A. Deutsch (Department of Auditory Research, Austrian Academy of Sciences, Liebigg. 5, A-1010, Vienna, Austria) and R. Steger (Department of Psychology, University of Vienna, Vienna, Austria)

Two general principles of pitch perception, analytic mode resulting in spectral pitch and synthetic mode resulting in virtual pitch have been investigated [E. J. Terhardt, Acoust. Soc. Am. 55, 1061-1069 (1974) I. It is assumed that virtual pitch is a product of Gestalt-perception, associated with some kind of learning process. If this concept is valid, it can be distinguished between spectral-pitch listeners (type D) and virtualpitch listeners (type ID. Complex tones consisting of components (a) $220,330,440,550$, and $660 \mathrm{~Hz}$ presented to the left ear and 550 and $660 \mathrm{~Hz}$ to the right ear were followed by (b) $200,300,400,500$, and $600 \mathrm{~Hz}$ left and 200,400 , and 600 right, which fused partially. The subjects were asked to decide whether pitch (a) or (b) was higher. Type I listeners responded (b) due to the spectral pitch of the corresponding Iateral positions of the partials in the head, whereas type II listeners responded (a) referring to the missing fundamental of $110 \mathrm{~Hz}$. In a second run the subjects were asked to pay specific attention to the different lateral positions too and again type I listeners differed from type II. The results are discussed in terms of Gestalt-rules and theory of pitch perception. [Work supported by AKG, Vienna.]

FRIDAY, 19 NOVEMBER 1976

GOLDEN WEST ROOM, 2:00 P.M.

\title{
Session WW. Speech Communication VIII: Perception II; Dichotic Listening, Miscellaneous
}

\author{
Fredericka Bell-Berti, Chairperson
}

Haskins Laboratories, New Haven, Connecticut 06510

\section{Contributed Papers}

\section{$2: 00$}

WW1. Abstract withdrawn.
Donald Wilson, and Michael S. Gazzaniga (Department of Psychology, State University of New York at Stony Brook, Stony Brook, NY 11794)

One current view of the mechanism underlying the ear advantage in dichotic listening has two components. The first is the relative superiority of contraolateral over ipsilateral auditory pathways, the second, the supression of ipsilateral information. While the notion of superiority simply implies an advantage for contralateral information, supression suggests that the information is unavailable. Three commissurotomy patients were given dichotic tests using consonantvowel syllables and digits, as well as a binaural fusion test, in order to evaluate their ability to use information presented to the ear ipsilateral to the language hemisphere. Consonantvowel syllables produced complete or near complete extinction of left ear information, while the digits test revealed a substantial increase in the use of left ear information. The fusion task produced performance greater than what would be expected by probability summation of the performance of each ear alone. The data suggest that in the absence of the corpus callosum, information from contralateral and ipsilateral ears can be integrated. Furthermore, the inability of the subjects to use information presented to the ipsilateral ear appears to be a function of the degree to which the competing items are acoustically similar, rather than a general supression mechanism.
WW2. Dichotic listening in split brain subjects: Ipsilateral suppression reconsidered. Sally P. Springer, John Sidtis, 
WW3. Left ear dichotic suppression following partial callosal section. M. L. Lenhardt (Medical College of Virginia of Virginia Commonwealth University, Richmond, VA 23298)

CV syllables (kindly provided by Kresge, LSU) were presented dichotically to a 13-year-old boy prior to and post surgical section of the majority of his corpus callosum. Preoperatively no ear advantage was observed; however, after callosal section a clear right ear superiority emerged. Correct performance approximately doubled for the right ear and fell to change for the left ear. Voiceless CV's were reported $\mathrm{Col}^{-}$ rect more often than voiced, both prior to and post surgery. In addition, alveolars were more often reported correctly than labials, which in turn are reported more correct than velars. His single correct response performance was highest when pairs shared voicing preoperative and lowest when pairs shared place. Postoperatively sharing place still resulted in the lowest percent correct; however, performance was highest when pairs shared neither place nor voice. An analysis of pairs resulting in errors revealed that for the (1) voiceless/ voiceless condition $16.6 \%$ errors occurred pre; and postoperatively, (2) voiced/voiced condition $21.0 \%$ errors occurred preop and $25.0 \%$ postop. ; (3) voiceless/voiced condition $64.4 \%$ errors occurred preop and $58.4 \%$ postop. Thus error pattern did not change after section. These data suggest that callosal section results in suppression of the ear countralateral to the speech processor by removing the interhemisphere relay route.

\section{$2: 30$}

WW4. Noninvariance of the ear advantage in dichotic listening. Charles Speaks, Nancy Niccum, and Edward Carney (Speech Research Laboratory, University of Minnesota, Minneapolis, MN 55455)

Listeners received 50 dichotic trials of both natural and synthetic CV syllables, and marked two responses for each of 30 syllables pairs per trial. The paired responses were distributed as a bivariate normal; left and right-ear scores had equal variance $(\sigma=8 \%)$ and were uncorrelated $(r=-0.02$ for synthetic; +0.15 for natural). Percent ear advantage also was distributed normally with a standard deviation about equal to the mean (synthetic, $\bar{X}=12.9 \%, \sigma=11 \%$; natural, $\bar{X}=9 \%$, $\sigma=10 \%)$. Thus, listeners with a mean REA evidenced many trials with either no ear advantage or an LEA (29\% natural; $19 \%$ synthetic). Variability in left and right-ear scores is assumed to arise from random, uncorrelated, time-varying "noise" in pathways from each ear. The ear advantage, left or right, is modeled as a function of the $\mathrm{S} / \mathrm{N}$ ratio in the respective pathways. The ear advantage is not invariant and, by this account, may not always be indicative of unilateral hemispheric specialization for speech processing. [Supported by PHS Grant No. NS-12125.]

$$
2: 40
$$

Ww5. Dichotic lag effect and right-ear advantage in children 7 to 15 . P.J. Mirabile, * R.J. Porter, Jr, , * L. F. Hughes, * and C.I. Berlin* (Kresge Hearing Research Laboratory, Dept. Otorhinolaryngology, LSU Medical Center, New Orleans, LA 70112)

Children aged $7,9,11,13$, and 15 years were tested on their ability to identify simultaneous and time-staggered dichotic consonant-vowel (CV) stimuli. At each age level, 15 males and 15 females listened to 300 dichotic trials, 60 trials at each of five onset asynchronies: $0,15,30,60$, and $90 \mathrm{msec}$. Simultaneous-onset results confirmed earlier dichotic findings in children: age-related performance increases and superior right-ear performance. In addition, the data revealed a lag effect with temporally asynchronous stimuli in a pattern similar to adults. However, the lag effect in younger children was more pronounced at $90 \mathrm{msec}$ than at the $30 \mathrm{msec}$ usually seen for adults. [Supported by NIH.]

*Also at University of New Orleans, Dept. of Psychology, Lakefront, New Orleans, LA 70122.
WW6. Dichotic and monotic masking of CV's by secondformants with different transition stating values. R. G. Whitaker and R.J. Porter, Jr. (Kresge Hearing Research Laboratory, Department of Otorhinolaryngology, LSU Medical Center, New Orleans, LA 70112 and Department of Psychology, University of New Orleans, Lakefront, New Orleans, LA 70122)

Subjects identified synthetic, two-formant, voiced stopvowel "targets" selected from an 11-step [bæ]-[gæ] continuum. Three targets were selected from the center of the " $b$ ", " $d$ " and " $g$ " categories, the two others from near category boundaries. Targets (presented at $50 \mathrm{~dB}$ ) were simultaneously paired with eleven second-formants ("bleats") extracted from the CV continuum stimuli. Pairs of targets and bleats were presented, dichotically and monotically in blocks, in a within-subjects design. Overall intensity of the bleat relative to the target was varied over blocks in $6-\mathrm{dB}$ steps from 0 to $-24 \mathrm{~dB}$. Subjects' performance varied with the nearness of the targets to boundary and the position of the bleat along the continuum; greatest interference occurred with the highest bleat intensities. Dichotic results tended to show a greater sensitivity to the "phonetic relevance" of the bleats and targets as defined in terms of their places on the continuum whereas monotic results tended to reflect more factors such as "ener gy averaging" in the $\boldsymbol{F}_{2}$ region. [Supported by NIH.]

$$
\text { 3:00 }
$$

WW7. Difference limens for formant frequencies of steadystate and consonant-bounded vowels. Paul Mermelstein and Hollis Fitch (Haskins Laboratories, New Haven, CT 06511)

Difference limens of formant frequencies were measured for two steady-state vowels and the same vowels in symmetric stop-consonant contexts. The stimuli were generated using a computer-programmed synthesizer and the formant frequency parameters were held constant or adj 1 sted to symmetric cubic functions of the time difference from the temporal center of the syllable. The difference limens (DL) for the time-varying consonant-vowel-consonant (CVC) stimuli were found to be significantly larger than those for the steady-state vowels, sometimes exceeding $10 \%$ of $F_{1}$ or $F_{2}$. In some cases the DL for the second formant is found to be larger in the direction of expected formant-shift due to consonantal coarticulation than in the reverse direction. The difference in DL values in and out of context has, at least partially, an auditory origin. Additionally, the phonetic decoding of the CVC stimuli may introduce information loss from auditory short-term memory. Implications for speech coding applications are discussed. [Supported in part by NICHD Grant HLI-01994.]

$$
3: 10
$$

WW8. Distinctive feature analysis of binaural versus monaural consonant perception as a function of signal to noise ratio. A Yonovitz (Speech and Hearing Institute, University of Texas Health Science Center at Houston, Houston, TX 77025), C. L. Thompson (University of Houston, Houston, TX 77005) and Priscilla C. Dickenson and J. T. Lozar (Speech and Hearing Institute, University of Texas Health Science Center at Houston, Houston, TX 77025)

Electret microphones inserted in the ear canals were used to binaurally record noncorrelated noise from two speakers equidistant at $\pm 45^{\circ}$ azimuth in an anechoic chamber. Twentyone CV's (with "a) were similarly recorded at $0^{\circ}$ azimuth. Various signal to noise ratios were olitained by mixing each binaural recording. These recordings maintained the effects of head shadow, pinna, etc. At each signal to noise ratio subjects wrote their response to the 30 presentations of each $\mathrm{CV}$ binaurally and monaurally. Confusion matrices were constructed. Data were analyzed in terms of consonant confusions and feature intelligibility. Multivariate analysis indicated differences in the contribution of the various features when binaural and monaural data were considered and also revealed changes in this pattern as a function of signal to noise ratio. 
$3: 20$

Ww9. Comparative evaluation of the $A X$ and two $A B X$ procedures. R.E. Pastore, C.J. Friedman, and K.J. Baffuto (Psychoacoustics Laboratory, Department of Psychology, SUNY-Binghamton, Binghamton, NY 13901)

The ABX procedure, which has been used extensively in speech perception research, provides a great deal of face validity. Unlike the classic same-different (or AX) procedure, each member of the pair of stimuli to be discriminated are presented on every trial. Furthermore, in theory, the estimates of discriminability are relatively free from response bias. Recently, this procedure has received considerable criticism for being contaminated, on a theoretical basis, by memory effects and thus providing diminished estimates of the discriminability between stimuli. In a series of experiments, we compared (a) the typically employed "random" ABX procedure (random relative relationship between $A$ and $B$ ), (b) a "fixed" ABX procedure (fixed relative relationship between $A$ and $B$ ), and (c) the same-different ( $A X)$ procedure in terms of psychometric functions and isosensitivity contours using an intensity discrimination task. The results are discussed in terms of the relative sensitivity of the procedures and their susceptibility to contamination by response bias.

FRIDAY, 19 NOVEMBER 1976

SUNSET ROOM, 2:00 P.M.

\title{
Session XX. Musical Acoustics IV
}

\author{
J. Backus, Chairperson \\ Department of Physics, University of Southern California, Los Angeles, California 90007
}

\section{A. Teaching the Physics of Sound II}

\section{Invited Papers}

\author{
2:00
}

XX1. Teaching musical acoustics to the musician-problems and questions. Robert E. Kelly (Physics Department, University of Mississippi, University, MS 38677)

Some problems and possible solutions associated with teaching musical acoustics specifically to musicians are discussed, along with some typical questions encountered. The topics are considered from a dual viewpoint: the physicist knowledgeable in music and a musician knowledgeable in physics. Some of the subjects considered are vibratos, tuning, terminology, transposition and algebra, scales, translation between scientific and musical language, choice of instruments and personality, and problems in communication.

$$
2: 30
$$

XX2. A musicologist's view of the teaching of musical acoustics. Edward L. Kottick (School of Music, The University of Iowa, Iowa City, IA 52242)

The teaching of musical acoustics has been dominated by those trained in scientific and technical fields. Many of these people are keenly interested in music and a good number of them are expert performers. But few of them are aware of the body of theoretical and historical knowledge about music that traditionally has been a part of the discipline of musical acoustics. As a result, some acoustical phenomena have been poorly understood and consistently misinterpreted. Scales, temperament, and the evolution of musical instruments are three of the se areas. Each can be explained more satisfactorily when music history, music theory, musical style, and organology are taken into account.

\section{Contributed Papers}

$3: 00$

XX3. An interdepartmental musical acoustics course. Donald E. Hall (Physics Department, California State University, Sacramento, CA 95819) and Gaylen Hatton (Music Department, California State University, Sacramento, CA 95819)

We believe it is important not to slight either music or physics in teaching a musical acoustics course. We are currently offering a course with two lectures (by G. H., with some informal participation by D. H.) and one coordinated lab/activity session (by D.H.) each week. We will discuss course organization, activity topics, advantages, and disadvantages of this plan. As an important component of the activities, each student works on an independent project and presents a report to the class. We conclude (1) that inclusion of experiments and activities as well as demonstrations is highly preferable to lectures alone, and (2) the course is more valuable and interesting when the teacher(s) can provide real expertise in both music and physics. 
3:15

XX4. Musical performance modification in differing acoustic environments. D. B. Harrison (Department of Integrated Studies, University of Waterloo, Waterloo, Ontario, Canada N2L 3G1)

By modifying their techniques, musicians are able to adapt performances to a variety of acoustic environments. Under some circumstances a musician is able to convey to the listener a property of the sound field which is nonexistent. Specific techniques practiced by organists are discussed and illustrated with recorded examples. The logic of many of these practices devcloped in the past may be seen in the light of modern acoustic and psychoacoustic knowledge. (It is suggested that as a supplement to courses in musical acoustics, material oriented more towards the performer's viewpoint, material such as this, might entice many musicians to learn more of the science of their art.)

\title{
B. General
}

\section{$3: 30$}

XX5. Peruvian whistling bottles. Steven Garrett (Department of Physics, University of California at Los Angeles, Los Angeles, CA 90024) and Daniel K. Stat (Museum of Cultural History, University of California at Los Angeles, Los Angeles, CA 90024)

Measurements were made of the frequency and intensity of 73 pottery "whistling bottles" encompassing a 200 -year period from 500 B.C. to $1550 \mathrm{~A}$. D. representing nine pre-Columbian cultures. We have found the whistles group acoustically by culture. The bottles are currently regarded by anthropologists as utilitarian liquid containers with the whistle providing an amusing method of venting to facilitate the passage of air during the pouring and filling of liquids. We are suggesting an alternative interpretation of the bottles as ritual artifacts specifically produced as whistles. We base this interpretation on thd close physical examination of a large sample and particularly on the pronounced effect of low frequency difference tones. If two or more whistles from a single culture are played simultaneously, difference tones are created within the ear due to the nonlinear interaction of their high-intensity tones.

\section{$3: 45$}

XX6. Impedance calculations for musically useful horns. J. D. Dudley and W.J. Strong (Department of Physics and Astronomy, Brigham Young University, Provo, UT 84602)

A numerical method for calculating input impedance of a horn of arbitrary shape is described. The horn is approximated by a sequence of cylindrical sections, and an assumption of plane waves is made. The input impedance is calculated as a function of frequency for various bore shapes and sizes, including cones, cylinders, cone-cylinder combinations, and other Bessel horns, and resonant frequencies are determined. The results are compared to those obtained by analytical methods and to experimental measurements which have been reported in the literature. The effect $s$ of radiation impedance are discussed, and the relevance of these calculations to the study of musical wind instruments is considered.

$$
4: 00
$$

XX7. Trumpet acoustics: Correcting ntonation faults. W. T. Cardwell, Jr. (Chevron Research Company, La Habra CA 90631)

Calculations are possible of the locations and sizes of constrictions and enlargements that may be put into a trumpet air column for correcting intonation errors of the trumpet. In certain respects, and to at least a useful approximation, the trumpet behaves as a simple, closed-open, cylindrical resonator, and perturbation theory appliec to a closed-open cylinder model permits the calculations of the locations and sizes of the constrictions and enlargements. The correctoramplitude versus distance function is a Fourier transform of the experimentally determined intonation-error versus mode function. An interesting experiment, making use of the theory, is the raising of the (usually) flat fifth mode without significantly affecting any of the other playing modes.

\section{$4: 45$}

XX8. Investigation of scaling law models for a rank of stopped diapason pipes. Theoren P. Smith, II (Naval Research Laboratory, Washington, DC 20375) and S. A. Elder (Physics Department, U.S. Naval Academy, Arnapolis, MD 21402)

The acoustical characteristics of a rank of closed diapason pipes have been investigated experimentally to provide a basis for testing theories of scaling laws. Neasured quantities determined for each pipe include pipe dimensions, sounding frequency, radiated sound pressure, jet speed at the lower lip, phase of jet crossing at upper lip, and pipe $Q$ as a function of amplitude. Comparison with current models of organ-pipe mechanism will be attempted.

\section{Session YY. Noise VIII: Analysis and Reduction of Aircraft Noise Sources}

\author{
John S. Gibson, Chairperson \\ Lockheed-Georgia Co., Marietta, Georgia 30063
}

\section{Contributed Papers}

$2: 00$

YY1. Some aspects of flow noise suppression. Abraham $C$. Peter (A.C. Peter \& Associates, 6481 Orangethorpe Ave., Buena Park, CA 90620)
Some aspects of flow noise suppression are discussed on the basis of the processes underlying the physics of flow noise formation (A.C. Peter, J. Acoust. So:. Am. 59, S92 (A) (1976) ]. It is shown that, for a given fluid, the efficiency of noise emission is a function of the frictional temperature rise 
attained by the frictional disturbance front. Accordingly, an early collapse of this disturbance front will cause a reduction of its lifetime $\left(i_{0} e_{2}\right.$, the time elapsed from the formation of the disturbance until its ensuing collapse). This, in turn, will cause a reduction of the frictional temperature buildup with the resultant decrease in the efficiency of flow noise emission. It is also shown that such early collapse of the disturbances may be induced by decreasing their linear scale. An illustrative application of these principles to the physical action of a multinozzle jet engine suppressor is made. It is shown that by forcing the jet exhaust to pass through a cluster of smaller nozzles, while keeping the velocity of efflux unchanged, the decreased scales of the disturbances induce a faster collapse, thus, decreasing the efficiency of flow noise emission. The device is also shown to shift the peaks of noise emission from lower to higher frequency ranges. The principles may be extended to other jet noise suppression devices which tend to "break up" the flow in the dissipative region.

$$
2: 15
$$

YY2。 Unified acoustic theory and its application. M。C.C. Tsao (Acoustics and Noise Control Research, Westinghouse Electric Corp., Pittsburgh, PA 15235)

The role of acousties theory in Noise Control Research is reviewed. The classical sound theory of $\partial^{2} \rho / \partial t^{2}-c^{2} \partial^{2} \rho / \partial x_{i}^{2}$ $=0$ which deals only with the wave propagation problem is compared with the unified acoustic theory derived by Lighthill, $\partial^{2} \rho / \partial t^{2}-c^{2} \partial^{2} \rho / \partial x_{i}^{2}=\partial Q / \partial t-\partial F_{i} / \partial x_{i}+\partial^{2} T_{i j} / \partial x_{i} \partial x_{j}$, which deals with the sound propagation problems as well as the sound generation problems. With this unified theory, the problems of fan noise, axial compressor noise, jet noise, flow noise, and turbulent noise all become apparent. The unified theory clearly illustrates the limitation of the nearfield and farfield sound measurement that can be confidently performed. Examples of various types of noise sources and their radiation directivity will be discussed.

\section{$2: 30$}

YY3. Noise and vibration ride comfort criteria. Thomas $K$. Dempsey, Jack D. Leatherwood, and Sherman A. Clevenson (Human Response Section, Noise Effects $\mathrm{Br}$ anch, Acoustics and Noise Reduction Division, NASA-Langley Research Center, Hampton, VA 23665)

A program is underway at Langley Research Center to develop a comprehensive ride quality model based upon the various physical and psychological factors that most affect passenger ride comfort. Two of the most important factors, namely vibration and noise, were studied in a previous investigation in which the relative contribution of each factor to overall passenger discomfort was determined. This earlier study utilized a category scale to elicit responses from subjeots exposed to combined sinusoidal vibration and octave band noises. The present study is an extension of this work and used a magnitude estimation procedure to obtain subjective responses to combined noise and vibration where the vibration stimuli now included random vibrations. The specific purposes of this paper are to (1) determine the absolute contribution of noise and vibration to passenger discomfort in terms of the discomfort units associated with the ride quality model, and (2) determine if passenger discomfort responses to combined noise and vibration depend significantly upon the nature of the vibration stimuli, i. e., deterministic as opposed to random. The stimuli for this study consisted of octave bands of noise centered at 500 and $200 \mathrm{~Hz}$ and vertical vibration composed of either $5-\mathrm{Hz}$ sinusoidal vibrations or random vibrations centered at $5 \mathrm{~Hz}$ and with a $5-\mathrm{Hz}$ bandwidth. The noise stimuli were present at levels ranging from ambient to $95 \mathrm{~dB} A$ and the vibration stimuli at levels ranging from 0.02 to 0.13 $g_{\mathrm{rma}}$.

\section{$2: 45$}

YY4. Correlation measurements of airframe noise in a wind tunnel. W. C. Meecham and D. R. Regan (University of Cali- fornia, Los Angeles, CA 90032) and Warren Ahtye (NASAAmes Research Center, Moffett Field, CA 94035)

Preliminary airframe noise measurements have been performed on a 19-ft-high model consisting of a half-fuselage and one wing with a three-flat system (nine flaps total). Pressure taps were placed on the middle of the underside flap surfaces, with the flaps full deployed during the test. Farfield microphones were placed approximately 12 feet away from the flap system and were mounted about 10 feet above the $40 \times 80 \mathrm{ft}$. NASA-Ames wind tunnel floor. During these test, the overall sound pressure level in the wind tunnel varied from 100 to 113 $\mathrm{dB}$ for flow speeds ranging from 130 to $230 \mathrm{ft} / \mathrm{sec}$. Cross-correlations between hydrodynamic pressure fluctuations on the flap surface and farfield sound yield normalized cross-correlation coefficients of up to 0.2 . It is found that the hydrodynamic pressure fluctuation at the flap surface is of order $\frac{1}{2} \rho_{0} u^{\prime 2}$, where $\rho_{0}$ is the ambient gas density and $u^{\prime}$ is the turbulent fluctuation velocity, Using the relation between the correlation function and the far-field radiated sound pressure level, it is found that the flap region (correlated with the pressure tap) radiates at sound pressure levels ranging from 70 to $9: 3 \mathrm{~dB}$. A numerical factor yet to be evaluated probably reduces these levels by up to $10 \mathrm{~dB}$. It is significant that the radiated sound is correlated with the hydrodynamic pressure measured at the center of the flap surface thus casting some doubt on the often stated proposal that sound radiation takes place only at the trailing edge of the flap surface. Estimating the effects of all eddies radiating from the flap structure yields a total sound pressure level ranging from 80 to $90 \mathrm{~dB}$. Such a plane flying overheat at an altitude of $500 \mathrm{ft}$ would give $r$ ise to sound pressure levels ranging from 55 to $65 \mathrm{~dB}$ for the speeds encountered in the present work. It is interesting to note that the sound pressure levels measured from the center region of the flap are generally $20 \mathrm{~dB}$ below the tunnel background noise level (100-113 dB), hopefully establishing the usefulness of cross-correlation techniques within a wind tunnel.

\section{$3: 00$}

YY5. OTW noise correlation for several nozzle/wing geometries using a 5:1 slot nozzle with various external reflectors. U. von Glahn and D. Groesbeck (NASA-Lewis Research Research Center, Cleveland, $\mathrm{OH} 44135$ )

The aerodynamic design requirements of the nozzle boattail for OTW configurations are different for cruise and powered lift. For cruise, a small nozzle boattail angle $(<109)$ is required to minimize drag; while for powered lift, a large angle $\left(25^{\circ}-30^{\circ}\right)$ is needed to provide jet flow attachment to the lifting surfaces, A solution to these design requirements is to use an external flow-deflector downstream of the nozzle for powered lift and to store (retract) this deflector for cruise. For powered lift, however, the jet/deflector/wing noise transmitted to ground observers can be a problem. In the present paper, representative acoustic spectral data obtained from a model scale study of several OTW configurations with a $5: 1$ slot nozzle using various external deflectors are correlated in terms of deflector geometry and flow parameters. Variations in the deflector geometry include deflector size and deflector angle. In addition, geometry variations in flap setting and nozzle chordwise location are included. Three dominant noise sources are correlated: fluctuating lift noise, flap-trailing edge and deflector related noise, and jet mixing noise. Lift and thrust measurements obtained in the study for the various configurations are summarized.

YY6. Flyover noise reduction for general aviation aircraft. Angelo J. Campanella (ACCULAB, 3201 Ridgewood Dr., Columbus, $\mathrm{OH} 43220$ )

A survey of noise emission reduction techniques is presented for propeller driven aircraft. Most of the audible noise is generated by the propeller tip traveling with a local velocity near that of the speed of sound. The feasibility of mechanical design steps (larger geared propeller and longer landing gear) 
required to reduce this speed was investigated. It was found that only new aircraft designs can be so configured. Existing aircraft will continue to operate. The attrition rate of these existing aircraft ( $1 \%-3 \%$ per year) is small, so that certain operational methods are desirable to achieve quieter airport environs. Procedures of administrative control, operational rules, a noise abatement power adjustments are described which decrease the noise exposure experienced on the ground. Enlightened land planning by county and state officials is described. [Work supported by FAA.]

$3: 30$

YY7. Aircraft cabin noise barrier design and test. Angelo J. Campanella (ACCULAB, 3201 Ridgewood Dr., Columbus, OH 43220)

The cockpit and cabin of contemporary propeller driven lightplanes continue to suffer high ambient noise levels in flight. These noise levels are typically $85-95 \mathrm{~dB} A$. It was sought to typify and test lightweight barrier configurations in an attempt to achieve the $30 \mathrm{~dB}$ predicted performance [J.T. Howlett and D. A. Morales, J. Acoust. Soc. Am. 59, S64 (A) (1976)]. Several structural arrangements were tested as 12 in. $(30 \mathrm{~cm})$ square samples. Broadband transmission loss values of 20-25 $\mathrm{dB}$ were easily achieved, as opposed to considerably smaller values for thin aluminum panels. The barrier performance due to stiffness over and above that due to the mass law is clearly evident at lower frequencies. The achievable TL values are compared with known aircraft noise spectra, both inside the outside the cabin, to predict cabin quieting achievable with practical weight and cost constraints.

$$
3: 45
$$

YY8. Analog to Curie's sound formula for closed cavities. T. E. Burton (General Atomic Co. , TO-170, P. O. Box 81608, San Diego, CA 92138)

Although S.N. Curle's very useful formula for aerodynamic sound radiated from a rigid surface can be applied to closed fluid circuits by the method of images, this procedure is not practical for cavities of complex shape. Sound fields in such cavities are best described with standing waves. However, substitution of an orthonormal mode expansion into the inhomogeneous wave equation results in volume integrals of dilatation source terms for the generalized model loads [N. A. Cumptsy and D.S. Whitehead, J. Sound Vib. 18, 353-369 (1971) l. Since the dilatation field is usually not known, the formal solution is difficult to apply. This paper presents an expansion of the sound field in a cavity in terms of nonorthogonal functions which results in a coupled set of equations with the generalized loads in terms of surface pressures (as in Curle's derivation), plus quadrupole terms. When the equations can be conveniently uncoupled and the quadrupole terms neglected, this formalism is very useful. Some of the cavity and flow geometries for which these two simplifications can be made and discussed.

$$
\text { 4:00 }
$$

YY9. Interface wave model for deep cavity resonator oscillation. S.A. Elder (Physics Department, U.S. Naval Academy, Annapolis, MD 21402)
Using superposed elementary wave functions, an attempt has been made to model the oscillating wave displacement profile at the interface of a flush-mounted deep cavity resonator. It is found that, in order to obtain a match with experimental profile data for an air cavity, it is necessary to postulate the presence of upstream as well as dowistream-traveling waves. Surface wave amplitude is set by requirement of asymptotic agreement with particle displacement of acoustic field in cavity. Implications for cavity drive mechanism will be discussed. IWork supported by GHR contract with the Naval Ship Research and Development Center. 1

$$
4: 15
$$

YY10. Mathematical model for unpar-itioned-cavity resonators. J.W. Sullivan and M. J. Crocker (Herrick Laboratories, Purdue University, West Lafayette, IN 47907)

In muffler design, concentric tube resonators are often used to provide high-frequency attenuation. These resonators are constructed by shaping a rigid shell around a length of perforated tube, forming an unpartitioned cavity. Because of the length of both cavity and perforated tube, it is not possible to use the simple Helmholtz resonator tineory to predict the attenuation. Beginning with a one-dirrensional control volume, a mathematical model was derived which accounted for the wave motion in the cavity and the coupling between cavity and tube via the impedance boundary of the perforate. For impedance in the linear regime, a closed form solution for the Transmission Loss was obtained. With this restriction and for the case of zero mean flow, excellent agreement was obtained with experimental results. Linited parametric studies suggest that the performance of this type resonator is quite sensitive to the porosity of the perforate, and that unusually large bandwidths of attenuation may be obtained for select geometires. [Work supported by Arvin Industries.]

$$
4: 30
$$

YY11. Analytical and experimental studies of folded cavity duct acoustic líners. R.J. Beckemeyer and D. T. Sawdy (Nacalle and Noise Abatement Branch, The Boeing Company, Wichita, KS 67210)

Low-frequency noise suppression in flow ducts can be achieved without excessive acoustic liner backing depth if the backing depth if the backing volume is formed as a long shallow cavity, folded so as to lie along the duct axis. Initial studies of such configurations revealed that sound transmission characteristics could not be accurately predicted when locally reacting impedance models were used. Consequently, an extended reaction model was developed for folded cavity liners by use of eigenfunction expansion techniques. Accuracy of the model has been verified by comparing analytical and experimental results for liners mounted in 6 -in. -square flow duct. test facility. Several face sheet impedanees and cavity geometries were tested at Mach numbers of 0.0 and 0.2 and frequencies from 500 to $1000 \mathrm{~Hz}$. Good agreement was obtained between theoretical and experimental values of panel insertion loss as well as between analytical and measured duct centerline axial standing wave patterns. 


\title{
Session ZZ. Speech Communication IX: Clinical Disorders
}

\author{
Grace H. Yeni-Komshian, Chairperson \\ National Institutes of Health, Bethesda, Maryland 20014
}

\section{Contributed Papers}

$3: 30$

2Z1. Speech quality of deaf children. R. Conrad (Department of Experimental Psychology, South Parks Rd., Oxford OX1 3UD, England)

A brief review is given of the problems involved in, and solutions suggested for, assessing the intelligibility of the speech of deaf children. A study of 350 deaf children aged 15-16 is reported which uses a new test for the intelligibility of spoken words. Results, as a function of hearing loss, are compared with the intelligibility of spoken two-digit numbers and with teachers' ratings. In all cases the correlation with hearing loss is very high; the main effect occurring for hearing loss between $65-95 \mathrm{~dB}$. Two-digit numbers are significantly more intelligible to untrained listeners than are words, and the role of reading ability in this connection is examined. With hearing loss taken out there is a highly significant correlation between intelligibility of spoken two-digit numbers and prose comprehension. But there is little evidence that the intelligibility advantage of spoken numbers over spoken words read by a deaf speaker, is due to poor reading ability.

$3: 40$

ZZ2. Experiments on the intelliglbility of speech codes for the severely hearing impaired. S. E. Stewart, W. J. Strong, and E. P. Palmer (Department of Physics and Astronomy, Brigham Young University, Provo, UT 84602)

We have reported previously (San Francisco ASA meeting in November 1975) on the intelligibility and discriminability of a low-frequency formant speech does with both normal hearing and hearing impaired subjects. Since then we have endeavored to discover just how much degradation the speech suffers in the process of coding and at what stage of the coding it occurs. Using Form IV of the Diagnostic Rhyme Test as test material, we have presented a modified speech code (dealing with partials of the speech rather than formants as such) at various stages of coding to normal hearing subjects. (This was done to assess the adequacy of the code and to initially avoid training hearing impaired subjects.) The test is used in the usual "intelligibility" mode in which the subject hears a single stimulus and is asked to identify it as one member of a word pair for the following conditions: (1) speech directly as recorded, (2) speech low-pass filtered $(4.5 \mathrm{kHz})$ followed by analog-to-digital and digital-to-analog conversions, (3) speech synthesized from largest amplitude partials. In addition, the test is run in a "discrimination" mode in which the subject hears a pair of rhyming stimuli and is asked to judge whether they are the same or different for the above conditions and for conditions after low-frequency transposing by a factor of four to one. Work is in progress with hearing impaired subjects.

$$
3: 50
$$

ZZ3. The relationship between speech discrimination ability and self-assessed hearing-handicap of adults with sensorineural hearing losses as a function of reverberation and noise. D. Mason and C. W. Asp (Department of Audiology and Speech Pathology, University of Tennessee, Knoxville, TN 37916)

The relationships between the Modified Rhyme Test which was administered in four listening situations-quiet and +5$\mathrm{dB}$ signal-to-noise ratio with reverberation times of 0.2 and
0.5 sec-and hearing handicap as determined by the SelfAssessed Hearing-Handicap Scale was investigated in a sample of sensorineural hearing impaired subjects. Analysis of the data revealed that there was a significant correlation between self-assessed hearing handicap and MRT scores, but only when the test words were presented in a $+5-d B$ signal-tonoise ratio with a reverberation time of $0.2 \mathrm{sec}$. Changes in correlation coefficients were explained by the effects of noise and reverberation on speech-discrimination scores as a function of the severity of hearing handicap. Noise affected the speech-discrimination scores of the more severely handicapped subjects to a greater degree than the less severely handicapped subjects. The opposite was true when reverberation time was lengthened.

$$
\text { 4:00 }
$$

ZZ4. Influence of vowel environment on duration of consonants produced by normal hearing, hearing impaired, and deaf adult speakers. R. L. Whitehead (National Technical Institute for the Deaf, Rochester, NY 14623) and K.O. Jones (Brigham Young University, Provo, UT 84602)

The effect of vowel environment on duration of consonants in the speech of ten normal-hearing, ten moderately to severely hearing-impaired, and ten profoundly deaf adult males was investigated. Each subject uttered five consecutive repetitions of the VCV syllables /isi/, /asa/, /i $\int i /$, and /a $\int a /$. The hearing-impaired and deaf subjects congenital hearing losses and were able to intelligibly produce the syllables. Each VCV sample was recorded onto audio tape and then individually played into a visicorder which produced the acoustic wave. Durational differences for $/ s /$ and $/ \delta /$ resulting from the two final vowel conditions were examined for each of the three groups. Results indicated that for both the normal-hearing and hearing-impaired groups, there were significantly longer durations of the consonants in the final / $i$ / environment than in the final $/ a /$ environment. For the deaf subjects, the consonants in the final /a/ environment were significantly longer than in the final $/ \mathrm{i} /$ environment.

\section{$4: 10$}

ZZ5. Evaluating a two-channel lip-reading aid. Brian L. Scott and Carol Lee De Filippo (Central Institute for the Deaf, 818 South Euclid, St. Louis, MO 63110)

The lip-reading ald tested consisted of a vibratory channel and an electrotactile channel. The speech wave was low-pass filtered at $1000 \mathrm{~Hz}$ and presented to the palm via a Suvag vibrator and was high-pass filtered at $4000 \mathrm{~Hz}$ and delivered to the back of the hand by a bipolar pulse generator designed by Frank Saunders and built by R. M. Sachs. The electrotactile channel provides information about sibilants, bursts, and higher formants; and the vibratory channel provides rhythm, stress patterns, and other prosodic features. Subjects were two nor mally hearing adults listening to noise that masked the acoustic signal. Test materials consisted of nonsense syllables, words, sentences, and ongoing text, each set being used under three conditions: (1) lip reading alone, (2) lip reading plus vibrator, and (3) lip-reading, vibrator, and electrotactile stimulation. All tests showed that the vibrator with lip reading was superior to lip reading alone and that adding the electrotactile channel provided further benefit. The 
range of improvement in the vibrator-plus-lip-reading condition was $7-21 \%$. Electrotactile stimulation provided an additional $2-10 \%$ improvement.

$$
4: 20
$$

2Z6. Tactual speech reception using a cochlear model. D.W. Sparks, P. K. Kuhl, and A. E. Edmonds (Applied Physics Laboratory, University of Washington, Seattle, WA 98195)

A tactual speech aid employing an electrical analogue of the cochlea is being evaluated on normal and hearing-impaired listeners. The cochlea has 36 frequency channels spaced logarithmically between $90 \mathrm{~Hz}$ and $10 \mathrm{kHz}$. The power envelope is extracted from each channel and submitted to a processor where it is transformed into eight discrete intensity steps. The outputs from this processor, then, are displayed as an intensity by frequency matrix consisting of 288 points ( 8 intensity $\times 36$ frequency). This spectral "plot" changes, as the speech spectrum, in real time. The matrix itself consists of 288 silver electrodes and is applied to the abdomen. The electrocutaneous stimulation seheme employs biphasic pulses for zero net current flow; the stimulators are constant-current limited for safety and comfort. Vowel, consonant, and connected discourse recognition are compared in three modes; (1) tactual alone, (2) visual alone, and (3) tactual with visual. Results are diseussed in relation to data obtained from other tactual speech aids.

$$
4: 30
$$

ZZ7. Acoustic study of speech in Parkinson's disease. C. L. Ludlow (NINCDS, National Institutes of Health, Federal Building, Bethesda, MD 20014)

Parkinson patients' speech was studied to determine which aspects differ from normal aging adult speech and what changes occur in speech when patients are in different neuromotor states-dyskinesia and rigidity. Parkinson patients alternating between dyskinesia and rigidity during dopamine enhancement treatment were recorded in each state while performing speech-imitation tasks presented on a test tape. Fundamental frequency, sound pressure level, duration and rate of syllables, and phrases were measured from spectrograms and power level tracings of the same speech segments in each recording. In contrast with the normal controls, Parkinson patients' speech during both dyskinesia and rigidity was reduced in length of extended phonation, rate of alternating between on-off phonation, and latency of vocal response. For fundamental frequency, speaking rate, syllable duration, and sound pressure level the degree of variation was reduced. The only speech measure differing between rigidity and dyskinesia was a lower sound pressure level during rigidity. The results indicated that Parkinson's disease significantly impairs laryngeal function and that only speech intensity level is affected by dopaminergic induced changes in neuromotor state.

$4: 40$

ZZ8. Analysis of speech produced with an artificial larynx. H. B. Rothman (Department of Speech. University of Florida, Gainesville, FL 32611) and L. P. Goldstein (VA Hospital, Gainesville, FL 32602)

For those individuals unable to learn esophageal speech and for the period of time until adequate ezophageal speech is developed, the successful use of an artiticial larynx is of primary importance. The purpose of this paper is to present the results of an investigation which measured and analyzed the speech parameters of (1) rate, (2) duration, (3) pitch, (4) intensity, and (5) articulation of both good and poor artificial larynx users. In addition to the analysis of the above parameters, perceptual judgments of speech intelli; ibility and proficiency were obtained. The conclusions reached from this investigation have resulted in the establishmen: of criteria for training laryngectomized individuals in the use of an artificial larynx. The material presented can also be used as a diagnostic basis to determine why an individual is not funetionally intelligible us using the artificial larynx.

$$
4: 50
$$

ZZ9. Multidimensional classification of abnormal voices. Sadanand Singh (Speech and Hearing Institute, 1343 Moursund, Houston, TX 77030) and Thomas Murry (Veterans Administration Hospital, San Diego, CA 92161) and Margaret Sargent (Speech and Hearing Institute, Houston, TX 77030)

Twenty samples of voice disordered speakers were randomly selected from a library of 200 tape recordings to determine their perceptual attributes. Twenty-three subjects judged the similarity of the ordered pairs of these voices on a sevenpoint scale. One of the three groups of seven judges rated the hoarseness, breathiness, and nasality. The judged similarities were analyzed by INDSCAL. The physical measures of airflow, $F_{0}, F_{1}, F_{2}, F_{2} / F_{1}, F_{2}-F_{1}$, and the acoustic spectrum and the psychophysical measures of hoarseness, breathiness, and nasality were used to interpret a fve dimensional INDSCAL solution. Dimension 1 was explained by absence versus presence of periodicity and by fudgments of "hoarseness." Dimension 2 was explained acoustically by elevation versus nonelevation of $F_{2}$, and diagnostically by presence versus absence of mass lesion. Dimension 3 was explained physiologically by airflow rate and psychologically as "breathiness" and to a lesser extent, as "nasal.ity." Dimension 5 was interpreted acoustically in terms of speakers' $F_{0}$. The fivedimensional solution accounted for $50 \%$ of the variance in the data. 